\title{
Optimizing ultrasound detection for sensitive 3D photoacoustic breast tomography
}

\section{Wenfeng Xia}





\section{OPTIMIZING ULTRASOUND DETECTION FOR SENSITIVE 3D PHOTOACOUSTIC BREAST TOMOGRAPHY}


Thesis committee members:

$\begin{array}{lll}\text { Prof. dr. } & \text { G. van der Steenhoven } & \text { University of Twente (Chairman) } \\ \text { Prof. dr. ir. } & \text { W. Steenbergen } & \text { University of Twente (Promotor) } \\ \text { Prof. dr. } & \text { T. G. van Leeuwen } & \text { University of Twente / AMC (Promotor) } \\ \text { Dr. } & \text { S. Manohar } & \text { University of Twente (Assistant-Promotor) } \\ \text { Prof. dr. ir. } & \text { C. H. Slump } & \text { University of Twente } \\ \text { Prof. dr. } & \text { K. J. Boller } & \text { University of Twente } \\ \text { Prof. dr. ir. } & \text { N. de Jong } & \text { Erasmus University Medical Center } \\ \text { Prof. dr. } & \text { H. J. C. M. Sterenborg } & \text { Erasmus University Medical Center } \\ \text { Prof. dr. -ing. } & \text { G. Schmitz } & \text { Bochum University }\end{array}$

The work described in this thesis was performed at the Biomedical Photonic Imaging (BMPI) Group, MIRA Institute for Biomedical Technology and Technical Medicine, Faculty of Science and Technology, University of Twente, P.O. box 217, 7500 AE, Enschede.

The research is founded by the Agentschap NL Innovation-Oriented Research Programmes Photonic Devices under the HYMPACT Project(IPD083374).

Cover Design: Wenfeng Xia, and Johan C. G. van Hespen

Printed by: Proefschriftmaken.nl || Uitgeverij BOXPress

Published by: Uitgeverij BOXPress,'s-Hertogenbosch

ISBN: 978-90-365-1216-9

DOI: $10.3990 . / 1.9789036512169$

Copyright (c) 2013 Wenfeng Xia, All right reserved. No part of the material protected by this copyright notice may be reproduced or utilized in any form or by any means, electronic or mechanical, including photo copying, recording or by any information storage and retrieval system, without prior consent from the author. Contact the author atwenfunxia@hotmail.com 
This thesis has been approved by:

Prof. dr. Wiendelt Steenbergen University of Twente (Promotor)

Prof. dr. Ton G. van Leeuwen University of Twente / AMC (Promotor)

Dr. Srirang Manohar University of Twente (Assistant-Promotor) 


\title{
OPTIMIZING ULTRASOUND DETECTION FOR SENSITIVE 3D PHOTOACOUSTIC BREAST TOMOGRAPHY
}

\author{
DISSERTATION
}

to obtain

the degree of doctor at the University of Twente,

on the authority of the rector magnificus,

Prof. dr. H. Brinksma,

on account of the decision of the graduation committee,

to be publicly defended

on Thursday the $31^{\text {st }}$ of October 2013 at $16: 45$

by

Wenfeng Xia

Born on the $3^{\text {rd }}$ of March, 1983

in Daye, China. 
To Yijing

In memory of my grandfather 



\section{Contents}

List of Figures $\quad$ xi

List of Tables $\quad$ xix

1 Introduction: The Twente photoacoustic mammoscope 1

1.1 Introduction . . . . . . . . . . . . . . . . . 1

1.2 The Twente Photoacoustic Mammoscope . . . . . . . . . . . . . . . . 4

1.2.1 Ultrasound detector properties . . . . . . . . . . . . . 6

1.2.2 Light delivery system . . . . . . . . . . . . . . . . 6

1.2.3 System characterization . . . . . . . . . . . . 7

1.2.4 Reconstruction . . . . . . . . . . . . . . . . 9 9

1.3 Clinical studies . . . . . . . . . . . . . . . . . . . . . . . . . . . 10

1.3 .1 Case $1 \ldots \ldots \ldots \ldots . \ldots \ldots$

1.3 .2 Case $2 \ldots \ldots \ldots \ldots \ldots$

1.3 .3 Case $3 \ldots \ldots \ldots \ldots$. . . . . . . . . . . . . . . . 16

1.4 Future work: speed of sound tomography with PA . . . . . . . . . . 18

1.5 Conclusions . . . . . . . . . . . . . . . . . . . . . 20

2 Poly(vinyl alcohol) gels as photoacoustic breast phantoms revisited 27

2.1 Introduction . . . . . . . . . . . . . . . . . . 28

2.2 Materials and Methods . . . . . . . . . . . . . . . . . 30

2.2.1 PVA phantom preparation . . . . . . . . . . . . . . . 30

2.2.2 Temperature measurements . . . . . . . . . . . . . . . . 31

2.2.3 Reduced scattering coefficient assessment . . . . . . . . . . 31

2.2.4 Speed of sound and acoustic attenuation assessment . . . . . . 33

2.2.5 Microstructure of PVA gels . . . . . . . . . . . . . . . 35

2.3 Results . . . . . . . . . . . . . . . . . . . 36

2.3.1 Temperature measurements . . . . . . . . . . . . 36

2.3.2 Reduced scattering coefficient . . . . . . . . . . . . . 37 
2.3.3 Speed of sound and acoustic attenuation measurements . . . 39

2.3.4 Microstructures of PVA gels . . . . . . . . . . . . . . 39

2.4 Discussion . . . . . . . . . . . . . . . . . . . . . . . 40

2.5 Conclusion . . . . . . . . . . . . . . . . . . . . 44

3 An optimized ultrasound detector for photoacoustic breast tomography

3.1 Introduction . . . . . . . . . . . . . . . . . . . . 52

3.2 Design parameters ......................... 54

3.2.1 Sensitivity and acceptance angle . . . . . . . . . 54

3.2 .2 Center frequency . . . . . . . . . . . . . . 56

3.3 Materials and fabricated models . . . . . . . . . . . . . 57

3.3 .1 Materials ................... 57

3.3.2 Functional and test models . . . . . . . . . . . . . 57

3.3.2.1 First functional model . . . . . . . . . . . . 57

3.3.2.2 Test models for minimizing radial resonances . . . . 58

3.3.2.3 Second functional model . . . . . . . . . . . . . . 58

3.3.2.4 Final model . . . . . . . . . . . . . . . . . . . . . 59

3.4 Numerical and experimental methods . . . . . . . . . . . . . . . 59

3.4.1 Simulation methods used . . . . . . . . . . . . 59

3.4.1.1 1D KLM model . . . . . . . . . . . . . . . . 59

3.4.1.2 3D FEM model . . . . . . . . . . . . . . . . 59

3.4.2 Detector characterization methods . . . . . . . . . 60

3.4.2.1 Electrical impedance . . . . . . . . . . . . 60

3.4.2.2 Acoustic frequency response . . . . . . . . . . 60

3.4.2.3 Directivity . . . . . . . . . . . . . 61

3.4.2.4 Sensitivity and minimum detectable pressure . . . 61

3.4.3 Imaging quality simulation . . . . . . . . . . . . . . 62

3.5 Results . . . . . . . . . . . . . . . . . 63

3.5.1 First functional model performance . . . . . . . . . . . 63

3.5.2 Test models performances . . . . . . . . . . . . 65

3.5.3 Second functional model . . . . . . . . . . . . . 65

3.5.4 Final model . . . . . . . . . . . . . . . . 65

3.5.4.1 Frequency response . . . . . . . . . . . 67

3.5.4.2 Directivity . . . . . . . . . . . . 68

3.5.4.3 Sensitivity . . . . . . . . . . . . . 68 
3.5.5 Imaging quality . . . . . . . . . . . . . . . . . . 69

3.6 Discussion . . . . . . . . . . . . . . . . . . . 70

3.7 Conclusion . . . . . . . . . . . . . . . . . . . . . 72

4 A new acoustic lens material for large area detectors in photoacoustic breast tomography $\quad 79$

4.1 Introduction . . . . . . . . . . . . . . . . . . . . . . . 80

4.2 Materials and methods . . . . . . . . . . . . . . . . . . . . 81

4.2 .1 Lens materials . . . . . . . . . . . . . . . . . . . . 81

4.2.1.1 Stycast 1090SI . . . . . . . . . . . . . . . . . 81

4.2.1.2 Acrylic plastic (PMMA) . . . . . . . . . . . 82

4.2.2 The detector . . . . . . . . . . . . . . . . . 83

4.2.3 Material acoustic properties characterization methods . . . . . 83

4.2.3.1 Speed of sound and acoustic attenuation . . . . . . 83

4.2.3.2 Density and acoustic impedance . . . . . . . . . . 84

4.2.4 Detector performance characterization methods . . . . . . . 85

4.2.4.1 Directivity (simulation and experiment) . . . . . 85

4.2.4.2 Frequency response (simulation and experiment) . . 86

4.2.5 Acoustic lenses used in photoacoustic tomography experiments 86

4.2.5.1 Forward problem (simulation and experiment) . . . 86

4.2.5.2 Image reconstruction . . . . . . . . . . . . . . 88

4.2.5.3 Image contrast analysis . . . . . . . . . . . . . 89

4.3 Results . . . . . . . . . . . . . . . . . . . . . . . . 89

4.3.1 Material acoustic properties . . . . . . . . . . . . . . 89

4.3.2 Acceptance angle using the acoustic lenses . . . . . . . . . . . 90

4.3.3 Pulse-echo and frequency response . . . . . . . . . . . . . . . 90

4.3 .4 Imaging quality . . . . . . . . . . . . . . . . . . . . . . 92

4.4 Discussion . . . . . . . . . . . . . . . . . . . . . . . 93

4.5 Conclusions . . . . . . . . . . . . . . . . . . . . . 94

5 Design and evaluation of a laboratory prototype system for 3D pho$\begin{array}{ll}\text { toacoustic full breast tomography } & 101\end{array}$

5.1 Introduction . . . . . . . . . . . . . . . . . . . . . . . 102

5.2 Materials and Methods . . . . . . . . . . . . . . . . . . . . . . 104

5.2 .1 The system . . . . . . . . . . . . . . . . . . . . . . . 104

5.2.1.1 Light source and light delivery system . . . . . . . . 104

5.2.1.2 Ultrasound detector array . . . . . . . . . . . 106 
5.2.1.3 Data acquisition . . . . . . . . . . . 107

5.2.1.4 Detector frequency response . . . . . . . . . 107

5.2.1.5 Detector directional sensitivity . . . . . . . . . 108

5.2.1.6 Detector sensitivity and minimum detectable pressure 108

5.2.1.7 Detector inter-element electrical and mechanical crosstalk109

5.2 .2 Phantom experiments . . . . . . . . . . . . . . . 110

5.2.2.1 Detector scanning procedure . . . . . . . . 110

5.2.2.2 Phantoms . . . . . . . . . . . . . 110

5.2 .2 .3 Image reconstruction . . . . . . . . . . . . 111

5.3 Results . . . . . . . . . . . . . . . . . . . . . . . . . . . . 112

5.3 .1 Detector array performance . . . . . . . . . . . . 112

5.3.2 Spatial resolution of the system . . . . . . . . . . . 113

5.3.3 Sensitivity and imaging quality of the system . . . . . . . 115

5.3.4 Field-of-view of the system . . . . . . . . . . . . . . 115

5.4 Discussion . . . . . . . . . . . . . . . . . . . . 115

5.4 .1 Light delivery . . . . . . . . . . . . . . . . . . . . . . . . . . . . . . . . . . . . . . . . . .

5.4 .2 Ultrasound detection . . . . . . . . . . . . . . . 118

5.4.3 Multi-modality imaging system . . . . . . . . . . . . 118

5.5 Conclusions . . . . . . . . . . . . . . . . . . . . . . 119

6 Conclusions and outlook $\quad 127$

6.1 The ultrasound detector . . . . . . . . . . . . . . . . . 127

6.1.1 Bandwidth and sensitivity . . . . . . . . . . 127

6.1 .2 Directivity . . . . . . . . . . . . . . . . 132

6.2 Acoustic lens . . . . . . . . . . . . . . . . . . . 133

6.3 Recommendations for future PAMs . . . . . . . . . . . . . . . . 134

6.3.1 Excitation ........................ 134

6.3 .2 US arrays ......................... 135

6.3.3 Multi-modality imaging system . . . . . . . . . . . . 136

6.4 Outlook ........................... 136

$\begin{array}{lr}\text { Summary } & 139\end{array}$

$\begin{array}{lr}\text { Samenvatting } & 142\end{array}$

$\begin{array}{ll}\text { Acknowledgements } & 145\end{array}$

$\begin{array}{lr}\text { List of publications } & 147\end{array}$ 


\section{List of Figures}

1.1 Schematic overview of the Twente Photoacoustic Mammoscope (PAM). 4

1.2 (a) Signal trace recorded from the central element of the detector matrix following photoacoustic generation in India Ink solution, (b) Frequency response of the detector element obtained by Fourier transforming the photoacoustic transient, (c) Sensitivity of the single element of the US detector. Experimental data have been linearly fitted. The dashed black line is the noise level without averaging. The minimum detectable pressure (MDP) is $80 \mathrm{~Pa}$ and is marked with an arrow in the figure. . . . . . . . . . . . . . . . .

1.3 Flow chart of the photoacoustic examination preliminaries and procedure followed for the pilot patient studies. . . . . . . . . . . .

1.4 Signal trace of an element showing the photoacoustic signals arising the breast of Case 2. A large signal f produced at the breast surface; at a depth of approximately $15 \mathrm{~mm}$ from the illuminated surface the signal from the tumour is seen. . . . . . . . . . . . . .

1.5 (a) Craniocaudal x-ray mammogram of Case 1 and (b) ultrasound image. Both show a large tumor mass with well defined margins. In the x-ray image, the supposed region-of-interest (ROI) is overlaid. (c) The contour of the breast under compression in the PAM, with a trace of the ROI and possible location of the tumor. This is shown with respect to the detector position. (d) Maximum Intensity Projection (MIP) of the three-dimensional photoacoustic reconstructed data. A ring-shaped region of high intensity probably marks the tumor rim where blood vessels are in abundance. The image size is $46 \mathrm{x} 43 \mathrm{~mm}$. 
1.6 (a) Craniocaudal x-ray mammogram of Case 2 and (b) ultrasound image. No mass is seen in the x-ray, but ultrasound shows a suspect tumor with poorly defined margins. (c) The contour of the breast under compression in the PAM, with a trace of the ROI and possible location of the tumor. This is shown with respect to the detector position. (d) Isosurface rendering of the three-dimensional photoacoustic reconstructed data. The image size is 40 x $53 \mathrm{~mm}$. . . . . . . . . .

1.7 (a) Ultrasound image of an abnormality in the breast of Case 3. At a depth of approximately $15 \mathrm{~mm}$ is seen a $23 \mathrm{~mm}$ diameter cyst. This can be recognized by the anechoic properties of the object with posterior enhancement. (b) Isometric view of the MIP of reconstructed photoacoustic data from the ROI carrying the cyst: two ellipses are marked in the figure where the cyst is expected to be according to the relative ultrasound image. No absorbers are seen where the cyst is expected, down to a depth of $30 \mathrm{~mm}$. A cyst does not possess enhanced vascularization and will remain "photoacoustically silent." It is not known why intense signals are present at higher depths roughly 33

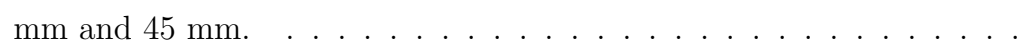

1.8 Schematic of the method for simultaneous imaging of speed-of-sound, acoustic attenuation and photoacoustics in a CT geometry. At S a carbon fibre is present in acoustic contact with the object at the origin and the curvilinear detector array at the far-end. Ultrasound is emitted from the carbon fibre when it is illuminated with pulsed light, which interacts with the object before being detected by the detector. A fan-beam projection is obtained. The data from such a projection is analyzed both in amplitude and time of arrival compared with a homogeneous reference measurement. Multiple projections around the object allow the reconstruction of the ultrasound transmission param-

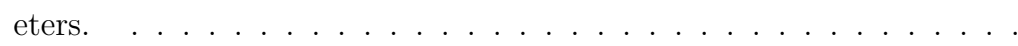

2.1 Schematic of poly(vinyl alcohol) gel prepared for speed of sound, acoustic attenuation, optical reduced scattering coefficient and microstructure measurements. . . . . . . . . . . . . . . . . . 
2.2 (a) Schematic of the oblique-incidence diffuse reflectance principle and setup. (b) Diffuse reflectance intensity map. (c) Diffuse reflectance profile (solid curve), calculated midpoint curve (dot) and light entry point (dash dot) at the position indicated in dash line in (b). . . . .

2.3 Reduced scattering coefficient $\left(\mu_{s}^{\prime}\right)$ at $784 \mathrm{~nm}$ for varying Intralipid $20 \%$ concentrations in water measured using the oblique-incidence diffuse reflectance setup. Our results are compared with $\mu_{s}^{\prime}$ values calculated from Van Staveren et al [22]. Each sample is measured 5 times, error bars represent standard deviations. . . . . . . . . . . . . .

2.4 Schematic of the setup for speed of sound and acoustic attenuation measurements. . . . . . . . . . . . . . . . . . . .

2.5 Scanning electron microscopy of (a) Outer surface of critical point dring processed sample and $(b)$ Liquid nitrogen processed sample. . . . . .

2.6 Temperature recorded at the surface (gray line) and $30 \mathrm{~mm}$ under the surface (black line) of a large PVA phantom during F-T cycles. . . . .

2.7 Measured $\mu_{s}^{\prime}$ distribution at $784 \mathrm{~nm}$ inside the large PVA phantom. Data points represent average $\mu_{s}^{\prime}$ value of 5 measurements from the plane $\mathrm{a}$, plane $\mathrm{b}$ and plane $\mathrm{c}$ of the phantom shown in Figure 2.1. The error bars represent the standard deviations. . . . . . . . . . . . .

2.8 Speed of sound and acoustic attenuation (at $5 \mathrm{MHz}$ ) at different depths from the surface to the bulk of large PVA phantom at $22.5^{\circ} \mathrm{C}$. The data points represent the average values from 10 measurements, error bars represent the standard deviations. . . . . . . . . . . . . .

2.9 Scanning electron microscope imaging of liquid nitrogen preprocessed specimens situated at (a) surface and (b) a deep lying cross-sections.

2.10 (a) Schematic of block III in Figure 2.1, (b) Pore sizes in the locations specified in (a), the error bars represent the standard deviations. (c) Pore density (black stars, left axis) and estimate of wall thickness (gray dots, right axis) for all specimens. (d) Pore diameter and wall thickness for grouped sample in depth. The error bars represent the standard deviations. . . . . . . . . . . . . . . . . . . . . 
2.11 In (a), an anatomically coronal T1-weighted MRI displays adipose (outer) and glandular (inner) tissue types. A two layer structured FEM mesh and source locations, created from (a), is shown in (b). Absorption and reduced scattering coefficient reconstructions in (c) are obtained without utilizing the internal structure of (b). In (d), MRI data guides a two-region parameter fitting algorithm. Relative to (c), resolution has improved and contrast has increased, showing higher absorption and scatter in glandular relative to adipose tissue. Reproduced from Ref. [40] with permission. . . . . . . . . . . . .

2.12 (a) Temperature of a large sample prepared using controlled FT recorded at surface, $3 \mathrm{~cm}$ under the surface and the set temperature for surface. (b) Optical reduced scattering $\mu_{s}^{\prime}$ distribution measured using the oblique-incidence diffuse reflectance setup for a large PVA sample prepared without (gray) and with (black) temperature controlled FT.

3.1 Schematics of a 2D photoacoustic tomography system showing the necessity for the acceptance angle to encompass the object for coherent signals detection from all angular position in performing reconstruction. 55

3.2 (a) Schematic of first functional model. (b) Schematic of subdiced second functional model and final model.(c) Photograph of the bare PZT samples with different lateral dimensions (label below). Two triangular samples are shown in the photograph, however, no related result is reported in this work. (d) Photograph of the final single-element model. 58

3.3 Schematics of the setups for detector frequency response measurements. (a) Transmit mode. (b) Pulse-echo mode. . . . . . . . . . . .

3.4 First functional model performance. (a) Electrical impedance of the first functional model measured with water load. (b) Measured transmission impulse response and frequency transfer function of the first functional model using a hydrophone. (time domain left axis, frequency domain right axis). . . . . . . . . . . . . . . . .

3.5 Test samples of PZT: measured and simulated electrical impedance in air with lateral dimensions (a) $5 \mathrm{~mm}$ x $5 \mathrm{~mm}$; (b) $4 \mathrm{~mm}$ x $4 \mathrm{~mm}$; (c) $3 \mathrm{~mm} \times 3 \mathrm{~mm}$; (d) $2 \mathrm{~mm}$ x $2 \mathrm{~mm}$; (e) $1 \mathrm{~mm} \times 1 \mathrm{~mm}$ and (f) $0.5 \mathrm{~mm} \times$ $0.5 \mathrm{~mm}$. No measured impedance available for $0.5 \mathrm{~mm} \times 0.5 \mathrm{~mm}$ PZT due to the practical limitations in manufacturing and measuring. . . . 
3.6 Second functional model performance: (a) Measured and simulated electrical impedance in water. (b) Measured and simulated pulse-echo ultrasound signal. The reflector is placed in the far-field of the detector. The time delay is removed. (c) Measured and simulated frequency response. . . . . . . . . . . . . . . . . . . .

3.7 Towards optimized final model: simulated pulse-echo signal and the frequency response of the sub-diced detector with different front- and back- matching layer thicknesses. (a) $t_{M L-F}: 0.58 \mathrm{~mm}, t_{M L-B}: 0.54$ mm. (b) $t_{M L-F}: 0.55 \mathrm{~mm}, t_{M L-B}: 0.54 \mathrm{~mm}$. (c) $t_{M L-F}: 0.70 \mathrm{~mm}$, $t_{M L-B}: 0.54 \mathrm{~mm}$. (d) $t_{M L-F}: 0.70 \mathrm{~mm}, t_{M L-B}: 0.48 \mathrm{~mm}$. . . . . .

3.8 Final model performance: (a) Measured and simulated far-field pulse of the final model time-shifted to origin. For the measurement, the pulse is probed using a calibrated broadband needle hydrophone in the far-field at distance $60 \mathrm{~mm}$, on center axis. (b) Measured and simulated frequency response of the detector. (c) Measured and simulated directional sensitivity. (d) Measured sensitivity and minimum detectable pressure (MDP) . . . . . . . . . . . . . . . .

3.9 (a) Initial pressure distribution used in the forward simulation. The gray dashed circle indicates the detector scanning positions. (b) Reconstructed image using signals detected by the final model, (c) by the transducer with $1 \mathrm{MHz}$ center frequency and $100 \%$ fractional bandwidth (Kruger et al 1999 [37]) and (d) by the transducer with 1.25 $\mathrm{MHz}$ center frequency and $200 \%$ fractional bandwidth (Andreev et al 2003 [19] and Ermilov et al 2009 [11]). (e)Profiles at position $\mathrm{X}=0 \mathrm{~mm}$ from the initial pressure distribution in (a) and reconstructed images from $(\mathrm{b}),(\mathrm{c})$ and $(\mathrm{d}) . \ldots \ldots \ldots$

4.1 Photograph of prepared PMMA and Stycast 1090SI samples: a $4.8 \mathrm{~mm}$ diameter hemispherical PMMA lens (left), a 5 mm diameter hemispherical Stycast 1090SI lens (middle), and two Stycast 1090SI blocks with dimensions of $15 \times 15 \times 2 \mathrm{~mm}$ and $15 \times 15 \times 4 \mathrm{~mm}$ (right) for acoustic properties characterization. . . . . . . . . . . .

4.2 Schematic of the setup for the acoustic transmission properties measurements. . . . . . . . . . . . . . . . . . . . . . . . .

4.3 Schematics of (a) directivity measurement setup and (b) pulse-echo signal and electrical-acoustical frequency response measurement setup. 
4.4 Schematic of the measurement configuration for the lenses used in a photoacoustic tomographic system. . . . . . . . . . . .

4.5 Measured acoustic transmission properties. (a) Speed of sound and (b) acoustic attenuation in Stycast 1090SI and acrylic plastic (PMMA) with frequency power law fitting as insertion for attenuation in low frequency regime. The values are measured at $21{ }^{0} \mathrm{C}$. Each data point represents an average of 5 measurements and error bars represent standard deviations of the measured values. . . . . . . . . . . . .

4.6 Directional sensitivity of the detector without a lens, with PMMA lens and with proposed lens: (a) simulated and (b) measured. . . . . . . .

4.7 Simulated (left column) and measured (right column) pulse-echo and frequency response of the detector: (a) without lens, (b) with PMMA lens, (c) with Stycast 1090SI lens, (d) without lens, (e) with PMMA lens and (f) with Stycast 1090SI lens. (a)-(c) are simulation results signals time-shifted to origin and (d)-(f) are measurement results. . .

4.8 Simulated (left column) and experimental (right column) PAT results for the described phantom: (a,i) Objects imaged by detector without an acoustic lens attached, (b,j) by detector with PMMA lens and (c, k) with Stycast 1090SI lens. Each image is normalized to its maximum intensity. (d, l) Axial intensity profiles along the trajectory indicated by dashed black line from (a)-(c). (e, m) Lateral intensity profiles indicated by dashed gray line from (i)-(k). (a)-(h) are simulation results, (i)-(p) are measurement results. Lateral resolution is improved by using both PMMA and Stycast lenses as expected, while axial resolution remains constant for all three cases. Due to limited bandwidth of the detector, ring-shape artifacts are presented around the objects. Those ring-shape artifacts are enhanced for objects imaged by detector with PMMA lens due to the ultrasound trapped inside the lens (indicated by arrows), while for the objects imaged by the detector with Stycast 1090SI lens, much weaker such artifacts are presented due to the tissue-like acoustic impedance of the lens material. . . . . . . . . . . . . . . . . .

5.1 Schematics of (a) the PAM-II laboratory prototype system and (b) the linear array detector.

5.2 Schematics of measurement setups used for studying (a) pulse-echo and frequency responses and (b) directional sensitivity. . . . . . . . . . . 107 
5.3 (a) Schematic of the tomographic system showing the relative positions between the scanning detector element and the phantom. (b) Schematic of phantom 1: a crossed-threads object embedded in Agar/Intralipid gel cylinder. (c) Schematic of phantom 2: five objects embedded in Agar/Intralipid gel cylinder. Detailed descriptions of the phantom and object properties are listed in Table 5.1. The lower part of the phantom is not shown in all three schematics. . . . . . . . . . . . 109

5.4 (a) Pulse-echo and frequency response, and (b) directional sensitivity of the detector element $5 . \ldots \ldots$. . . . . . . . . . . . . . . . . 112

5.5 (a) Sensitivity and minimum detectable pressure of the detector element 5. (b) Minimum detectable pressure for each element in the detector array. . . . . . . . . . . . . . . . . .

5.6 (a) Responses of all elements in the array displayed in different vertical scales for visualization. Electrical and mechanical crosstalk are well separated in time. (b) Peak-peak inter-element electrical and mechanical crosstalk of the linear array detector relative to the driven element. . . . . . . . . . . . . . . . . .

5.7 Reconstruction of phantom 1. (a) A top view maximum intensity projection (MIP) along the vertical axis (Z direction) with $100 \times 100 \mathrm{~mm}^{2}$ field of view. (b) A side view MIP along the $\mathrm{Y}$ direction with $60 \mathrm{x}$ $100 \mathrm{~mm}^{2}$ field of view. (c) An image slice at position indicated by dashed line in (a) showing cross-sections of the two threads (marked with " 1 ", and "2") at around $4 \mathrm{~cm}$ from the phantom surface. (d) A $3 \mathrm{D}$ rendering of the phantom showing a $110 \times 110 \times 70 \mathrm{~mm}^{3}$ field of view. (e) An axial profile crossing a sub-resolution object ("1") from (c) indicated by a dashed white line, and (f) A vertical profile crossing a sub-resolution object ("1") from (c) indicated by a dashed gray line. (g) An axial profile crossing a sub-resolution object ("2") from (c) indicated by a dashed white line, and (h) A vertical profile crossing a sub-resolution object ("2") from (c) indicated by a dashed gray line. . 
5.8 Reconstruction of phantom 2. (a) A top view maximum intensity projection (MIP) along the vertical axis (Z direction) with $70 \times 70 \mathrm{~mm}^{2}$ field of view. (b) A side view MIP along the X direction with $60 \times 70$ $\mathrm{mm}^{2}$ field of view.(c) A XY plane image slice at $20 \mathrm{~mm}$ depth from the phantom surface showing the reconstructed objects on this plane. (d) A XY plane image slice at $40 \mathrm{~mm}$ depth from the phantom surface showing the reconstructed objects on this plane. . . . . . . . . 116

6.1 Photoacoustic signal arriving at tissue surface from a $100 \mu \mathrm{m}$ spherical object (blood vessel contrast) at $5 \mathrm{~cm}$ depth with and without the taking into account of acoustic attenuation (AA). (a) Time domain signals. (b) Normalized spectrums. . . . . . . . . . . . . . . . . . 128

6.2 Characteristics of photoacoustic signals arriving at tissue surface from spherical objects with different diameters, located at different depths. Center frequency and bandwidth for (a) spherical objects with diameters from 0.1 - $10 \mathrm{~mm}$ located at 10 and $50 \mathrm{~mm}$ depth and (b) Object with diameters of $0.1,1$, and $10 \mathrm{~mm}$ located at various depth from tissue surface to $80 \mathrm{~mm}$. Solid lines represent center frequencies and error bars represent half bandwidths. (c) Peak pressures for tumors with diameter of $1 \mathrm{~mm}, 2 \mathrm{~mm}, 5 \mathrm{~mm}$ and $10 \mathrm{~mm}$. (d) Peak pressures for blood vessels approximated by spheres with diameter of $0.1 \mathrm{~mm}$, $0.2 \mathrm{~mm}, 0.5 \mathrm{~mm}$ and $1 \mathrm{~mm}$. . . . . . . . . . . . . . . 130

6.3 Schematics of a $2 \mathrm{D}$ photoacoustic tomography system showing the necessity for the acceptance angle to encompass the object for coherent signals detection from all angular position in performing reconstruction.132

6.4 Schematic showing the prospects of the future clinical version system. 135 


\section{List of Tables}

1.1 Optical properties of test materials for bandwidth and resolution assessment. ....................... 5

1.2 System spatial resolution . . . . . . . . . . . . . . . . . . 8

1.3 System maximum imaging depth from simulation . . . . . . . . . . 9

1.4 Patient studies . . . . . . . . . . . . . . . . . . . . . . 12

2.1 Validation of the oblique-incidence diffuse reflectance system. The sample is calibrated at $800 \mathrm{~nm}$ and measured at $784 \mathrm{~nm}$. Five measurements are taken for the sample. . . . . . . . . . . . . . .

2.2 Calibrated $\left(22^{\circ} \mathrm{C}\right)$ and measured $\left(22.7^{\circ} \mathrm{C}\right)$ speed of sound and acoustic attenuation values for the two calibrated samples. Temperatures were stable within $\pm 0.05{ }^{\circ} \mathrm{C}$ during the measurement time of 5 minutes. The mean and dispersions in measured thicknesses were used in the estimation. . . . . . . . . . . . . . . .

2.3 Our results compared with literature values for the optical reduced scattering $\mu_{s}^{\prime}$ in adipose and glandular tissue. . . . . . . . . . .

3.1 Properties of the materials used for the detector in 3D FEM simulations. Properties of the PZT material are from reference [40,41], and the properties of the matching and backing layers are from reference [41]. 56

3.2 Layer thicknesses of functional models (Figure 3.2(a) and (b)), each layer has a $5 \mathrm{~mm}$ x $5 \mathrm{~mm}$ square-shape surface. . . . . . . . . . .

3.3 List of US detectors used by different groups in the photoacoustic (thermoacoustic) systems for breast imaging. . . . . . . . . . . . 
4.1 Measured acoustic properties of lens materials (Stycast 1090SI and PMMA) compared with literature values for water. All values are for material at $21{ }^{0} \mathrm{C}$ and for frequency of $1 \mathrm{MHz}$. The longitudinal velocity, shear velocity and acoustic attenuation are used in 3D FEM simulations. . . . . . . . . . . . . . . . . . 86

5.1 Background and object properties of phantom 2. Their dimensions, distances from phantom surface (depths), optical reduced scattering coefficient $\mu_{\mathrm{s}}^{\prime}$, and optical absorption coefficient $\mu_{\mathrm{a}}$ are listed. The optical properties of normal and cancerous breast tissue are derived from Ref. [45] at $755 \mathrm{~nm}$ and human blood is derived from Ref. [46] at 800 $\mathrm{nm}$, where blood absorption is independent of the oxygen saturation level. . . . . . . . . . . . . . . . . . . . . . . . . 110

6.1 Optical, acoustic and thermo-elastic properties of healthy breast tissue and tumors used in the photoacoustic signal generation and propagation model. Optical properties are from Ref. [6] at $785 \mathrm{~nm}$ wavelength. The speed of sound, acoustic attenuation and optical scattering properties, and Grüneisen coefficient are assumed to be homogenously distributed in the model used. Healthy tissue, tumor and blood are modelled to have differences in optical absorption only. . . . . . . . . 


\title{
Chapter 1
}

\section{Introduction: The Twente photoacoustic mammoscope}

\author{
${ }^{1}$ Abstract
}

The Twente Photoacoustic Mammoscope (PAM) uses pulsed light at $1064 \mathrm{~nm}$ to excite photoacoustic signals. Detection is using a planar 590 element ultrasound sensor matrix. Image reconstruction uses a delay-and-sum beamforming algorithm. Measurements are performed in the forward mode, with mild compression of the breast against the detector with ultrasound coupling gel. We consolidate the most important specifications of the system. Further we discuss in detail the results of imaging two cases of infiltrating ductal carcinoma and one case of a cyst. We critically discuss the features of the present embodiment and present plans for its improvement.

\subsection{Introduction}

The current practice of imaging the breast for the detection and diagnosis of cancer suffers from shortcomings. The interactions of the probe energy - whether x-rays, magnetic field or ultrasound - with a tumour are not sufficiently specific or sensitive compared with non-pathological tissue. This results in occurrences of false positives and false negatives, which lead to psychological and physical morbidity, and mortality.

\footnotetext{
${ }^{1}$ This chapter has been published as: D. Piras, W. Xia, W. Steenbergen, T. G. van Leeuwen and S. Manohar, "Photoacoustic imaging of the breast using the Twente Photoacoustic Mammoscope: present status and future perspectives," IEEE J. Selec. Topic Quant. Electron 16, 730-739 (2010). Reproduced with permission.
} 
Further, though x-ray mammography is the gold standard imaging modality, x-rays are carcinogenic $[1,2]$. Ultrasound (US) and Magnetic Resonance Imaging (MRI) are restricted in their use to being secondary procedures owing to low sensitivity (US), poor specificity (MRI) and high-expense (MRI) [2].

Among the alternative techniques, optical imaging using near-infrared (NIR) light has attracted much interest [3-6]. Optical detection of cancer is possible because of the optical absorption contrast between tumors and healthy tissue $[4,7,9]$. When malignancy arises, a phase of rapid growth and invasion of the abnormal cells into surrounding tissues accompanies and follows angiogenesis - the formation of blood vessels within and around the tumor [10]. Hemoglobin $(\mathrm{Hb})$ is the main constituent of red blood cells and the strongest biological chromophore in the NIR spectral range [112]. The increased $\mathrm{Hb}$ concentrations bestow absorption to tumors, which can be around three times stronger when compared with healthy tissues [7].

Low-intensity electromagnetic waves in the visible and infrared regimes do not have ionizing related health hazards. Further, imaging with light is relatively inexpensive compared with techniques such as MRI. Additionally, chromophores in tissue have characteristic spectral features which allow them to be identified if spectroscopy is performed $[6,12]$. This feature provides a strong diagnostic potential to the use of light for medical imaging.

A major drawback of optical techniques is that in the visible-NIR spectral region light does not penetrate biological tissues in straight paths as x-rays do, but undergoes severe scattering [13]. Since these scattered photons are collected and analyzed for obtaining imaging data, all "purely optical" imaging methods have to contend with poor spatial resolution when large depths are probed.

Photoacoustic imaging has the advantages of optical imaging, but without the optical scattering dictated resolution impediment. In photoacoustics, when short pulses of light are used [14-16], absorption at certain tissue sites induces heating followed by rapid thermal expansion. This generates ultrasound waves that propagate through tissue, to be detected by acoustic transducers positioned at the surface [17]. The time-of-flight, amplitude and duration of acoustic pulses recorded on the tissue surface possess information regarding the location, absorption and dimensions of the source. By recording signals in a two-dimensional grid of detector positions, a threedimensional reconstruction of the absorber is possible. The advantage of this approach compared with purely optical imaging is that the spatial resolution is superior because of the low scattering experienced by ultrasound during propagation in soft tissue [16].

The main steps in photoacoustic imaging are: 
i) certain tissue structures absorb short pulses of NIR light;

ii) fast de-excitation results in thermalization and temperature rise in tissues;

iii) thermal expansion results in an initial pressure distribution in tissue;

iv) the pressure relaxes with the emission of acoustic waves which propagate to the surface of tissue;

v) the pressure transients are registered on the surface using ultrasound transducers at various spatial positions;

vi) the recorded data are used to reconstruct the initial pressure distribution as an image of the absorbing structures in tissue.

Photoacoustic imaging prototypes capable of breast imaging have been reported in $[16,19-23]$ as the Thermoacoustic Computed Tomography (TCT) system, Laser Optoacoustic Imaging System (LOIS) and Thermoacoustic / Photoacoustic Tomography (PAT / TAT) respectively.

The TCT prototype uses radio-frequency photons at $434 \mathrm{MHz}$ for excitation with three wide-bandwidth $1 \mathrm{MHz}$ planar arrays for detection. With this instrument entire coverage of the breast is possible during scanner rotation while mapping the ionic water content with a spatial resolution of 1-2 $\mathrm{mm}$ and depth of penetration of about $10 \mathrm{~cm}$. Recent clinical results are discussed in [22].

LOIS uses NIR laser pulses at $757 \mathrm{~nm}$ from an Alexandrite laser and $1064 \mathrm{~nm}$ from an Nd:YAG laser, sequentially illuminating the breast. Detection is with an arc-shaped 32 element array providing 2D and 3D maps of the optical absorption with a spatial resolution of $0.5 \mathrm{~mm}$ [19]. An overview of the clinical work has been given in $[20,21]$.

PAT/TAT is a recent system combining photoacoustic and thermoacoustic imaging into one hybrid device. The microwave $(3 \mathrm{GHz})$ source and the laser $(\mathrm{Nd}$ :YAG at $1064 \mathrm{~nm}$ ) source are alternatively switched on [23]. The ultrasound detectors are scanned around the breast collecting data at 360 degree angle positions. Clinical studies are yet awaited.

At the University of Twente we have followed a different approach [24-26,28,29,48]. The Twente Photoacoustic Mammoscope (PAM) uses a parallel plate geometry with the breast being mildly compressed against the detector. The system is based on a flat ultrasound detector with 590 elements and using $1064 \mathrm{~nm}$ light from an Nd:YAG laser. In a parallel plate geometry, photoacoustic images are comparable with those from x-ray mammograms. We review the present status of clinical studies and outline some plans for future work. 


\subsection{The Twente Photoacoustic Mammoscope}

When an absorbing sphere-like inclusion embedded in a turbid less absorbing medium is illuminated, the differential photothermal heating results in a pressure transient which propagates from the sphere as an ultrasound wave. Under the assumption of thermal and stress confinement, the pressure induced by photoacoustic irradiation is a bipolar pulse with amplitude and duration dependent, respectively, on the sphere's absorption coefficient and on its radius [30-32].

The choice of the irradiating wavelength is based on a compromise between light penetration properties on the one hand and a good contrast of vascularized tumors on the other. As discussed in literature [7,33]. in the wavelength range 1000-1100 $\mathrm{nm}$, breast tissue has a low effective attenuation coefficient with maximal optical penetration depths [7]. At $1064 \mathrm{~nm}$ the presence of local minima in the optical absorption spectra of water and lipids make this wavelength attractive for achieving deep penetrations in the post-menopausal breast. At $1064 \mathrm{~nm}$ however, the absorption by hemoglobin is low yielding lower tumor contrast. Though other wavelengths like $755 \mathrm{~nm}$ have been shown to be more effective in imaging contrast [34], we decided to choose for a better breast coverage with $1064 \mathrm{~nm}$ at the expense of contrast.

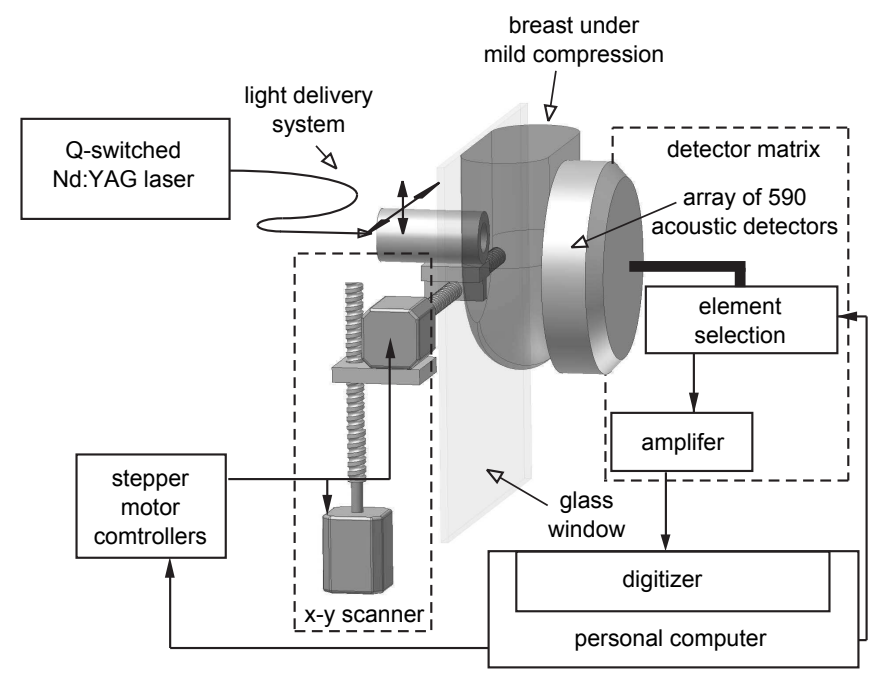

Figure 1.1: Schematic overview of the Twente Photoacoustic Mammoscope (PAM).

In the PAM $[24,25,28]$ the breast is slightly compressed between a window for laser light illumination and a flat array ultrasound detector. This configuration depicted 
in Figure 1.1, ensures a good acoustic contact between the breast and the detector when coupling gel is used between them; besides, this geometry is similar to that of $\mathrm{x}$-ray mammography and hence facilitates comparison with standard x-ray exams of the same breast.

The experimental setup involves a Q-switched Nd:YAG laser light (Brilliant-B, Quantel, Paris) at $1064 \mathrm{~nm}$, with $5 \mathrm{~ns}$ pulse duration and $10 \mathrm{~Hz}$ pulse repetition frequency. Temperature and stress confinement constraints for breast tissues are respected. The light beam scans breast regions with a 2D light delivery scan system. The photoacoustic process generates ultrasound waves which arrive at the ultrasound detector: detector elements are read into the PC on one channel of a dual channel digitizer (NI-5112, $100 \mathrm{MHz}, 100 \mathrm{MS} / \mathrm{s}, 8$ bit). A Labview program controls the scanning stage and the element selection. Reconstruction is performed with a delay and sum beam-forming algorithm [34] . The instrument is built into a hospital bed on which the patient lies prone with her breast pendant through the aperture into the scanner.

Table 1.1: Optical properties of test materials for bandwidth and resolution assessment.

\begin{tabular}{lll}
\hline \hline phantom & material & $\begin{array}{l}\text { optical properties } \\
(1064 \mathrm{~nm})\end{array}$ \\
\hline P1 & India Ink 50\% in water [25] & $\mu_{a}=214 \mathrm{~mm}^{-1}$ \\
\hline P2 & Poly(vinyl alcohol) gel using DMSO & $\mu_{a}=0.25 \mathrm{~mm}^{-1}$ \\
& dyed with Ecoline black [37] & \\
\hline P3 & $4 \%$ Intralipid 10\% stock [36] & $\mu_{s}^{\prime}=0.3 \mathrm{~mm}^{-1}$ \\
\hline M1 & Poly(vinyl alcohol) gel produced & $\mu_{a}=0.035 \mathrm{~mm}^{-1}$ \\
& using freeze/thaw method [37,38] & $\mu_{s}^{\prime}=0.5 \mathrm{~mm}^{-1}$ \\
\hline M2 & Poly(vinyl alcohol) gel using DMSO & $\mu_{a}=0.2 \mathrm{~mm}^{-1}$ \\
& dyed with Ecoline black with & $\mu_{a}=0.1 \mathrm{~mm}^{-1}$ \\
& contrast 7x, 4x, 2x [37,38] & $\mu_{a}=0.005 \mathrm{~mm}^{-1}$ \\
\hline \hline
\end{tabular}

${ }^{a}$ relevant optical properties are $\mu_{a}=$ absorption coefficient and $\mu_{s}^{\prime}=$ reduced scattering coefficient. 


\subsubsection{Ultrasound detector properties}

A PVDF sheet (110 $\mu \mathrm{m}$ thick, $90 \mathrm{~mm}$ diameter) has been used with gold electrodes forming the 590 elements, $2 \times 2 \mathrm{~mm}$ each, with $3.175 \mathrm{~mm}$ element pitch. An 18.6 $\mathrm{mm}$ thick layer of a proprietary polymeric material serves as support and protection to the PVDF sheet.

This polymer layer has acoustic impedance close to that of tissues. While the face of the sheet against the layer is metalized, the electrical contacts to the rear face electrodes are obtained by spring-loading conductive pins against the PVDF film; this spring system minimizes reverberations. Elements are grouped in 10 sectors of approximately 60 elements each serviced by an ASIC which buffers and amplifies the signals from elements. One element is activated at a time [25,29]. Time and frequency domain characteristics of the central element of the detector have been reported in Fig. 2. An acoustic impulse was generated by illuminating a highly absorbing specimen (P1 in Table 1.1). The Fourier transform of the acquired signal shows frequency content in the range $450 \mathrm{kHz}$ to $1.78 \mathrm{MHz}(130 \%-6 \mathrm{~dB}$ fractional bandwidth). This is then the frequency response of the system. The Minimum Detectable Pressure (MDP) of the detector is estimated by a substitution method using a $1 \mathrm{MHz}$ transmitting transducer and a calibrated hydrophone system (Precision Acoustics Ltd. Dorchester) with a $0.2 \mathrm{~mm}$ needle hydrophone (HPM 02/1) with known discrete frequency calibration curve. One element of the detector is activated and the transducer which insonifies this element has its excitation progressively reduced until the signal vanishes in the background noise. The pure noise is then measured separately when using minimal transducer input pressure. The transducer is then replaced by the hydrophone and the pressure to excitation transfer function is obtained. As showed in Figure 1.2, this method gives a precise noise value for calculating MDP and so provides a clear border between signal and noise, in this study, the MDP measured without any average of signals is $80 \mathrm{~Pa}$, the MDP value is reduced by a factor of 10 when taking 100 averages of the signals.

\subsubsection{Light delivery system}

Deep imaging using the detector requires at least 50-60 mJ per pulse at $1064 \mathrm{~nm}$ with at least 100 averages. Laser safety standards in the Netherlands (NEN-60825-1) permit a Maximum Permissible Exposure (MPE) of $30 \mathrm{~mJ} \mathrm{~cm} \mathrm{~cm}^{-2}$ per pulse for a pulse train of 100 pulses, for the laser class (1064 nm, $5 \mathrm{~ns}$ pulses, $10 \mathrm{~Hz}$ ) in use. To heed these limits while meeting minimum energy requirements, the laser beam is expanded 


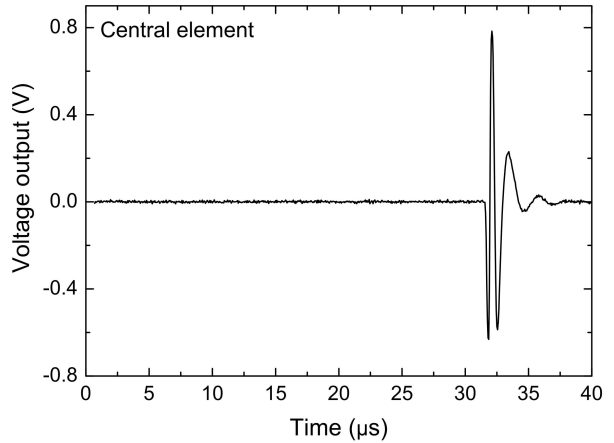

(a)

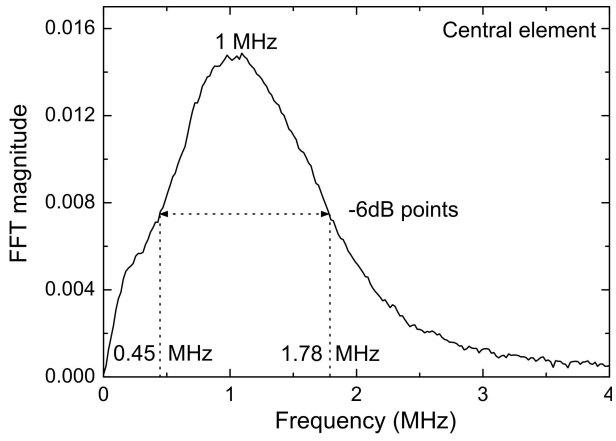

(b)

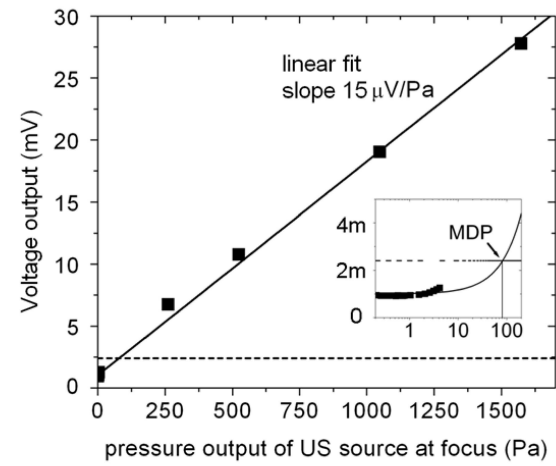

(c)

Figure 1.2: (a) Signal trace recorded from the central element of the detector matrix following photoacoustic generation in India Ink solution, (b) Frequency response of the detector element obtained by Fourier transforming the photoacoustic transient, (c) Sensitivity of the single element of the US detector. Experimental data have been linearly fitted. The dashed black line is the noise level without averaging. The minimum detectable pressure (MDP) is $80 \mathrm{~Pa}$ and is marked with an arrow in the figure.

to $16 \mathrm{~mm}$.

A custom system was developed, based on a geometry with 2 prisms and 2 movable joints, and described in detail in [25]. This delivery system forms the payload of an $\mathrm{x}-\mathrm{y}$ scanning stage so that the laser beam can be translated in steps, to illuminate the ROI of the breast, while at each position an antipodal element of the detector is activated.

\subsubsection{System characterization}

The system was characterized using a variety of samples which are consolidated in Table 1.1. 
Taking the maximum detectable frequency $\left(\mathrm{f}_{\max }\right)$ as $2.5 \mathrm{MHz}$, the smallest object that can be faithfully registered in the system [39-42] assuming $1490 \mathrm{~m} / \mathrm{s}$ speed of sound $(\mathrm{v})$ :

$$
r_{0 \min }=1.5 \frac{v}{f_{\max }}
$$

is calculated as $1.8 \mathrm{~mm}$ in diameter.

Ultrasound detector resolution depends on detector element size, number of elements, element pitch, directional sensitivity, detector to source distance, element frequency response [39-42] and on the image reconstruction algorithm. The assessment of the system's resolution was achieved by examining the point-spread function (PSF) using a $2 \mathrm{~mm}$ sized highly absorbing gel sphere (P2) suspended at a depth of $10 \mathrm{~mm}$ from the surface in Intralipid (P3). Images were obtained for $17 \times 17$ elements in the imaging area $52 \mathrm{~mm} \times 52 \mathrm{~mm}$. The size of P2 is deconvolved from the full width at half maximum (FWHM) of the image of P2 to get a quantitation of the PSF. The results of the resolution experiments are shown in Table 1.2. For 15 and $60 \mathrm{~mm}$ distances from the transducer surface, the lateral resolution ranged from 3.1 to $4.4 \mathrm{~mm}$ and the axial resolution from 3.2 to $3.9 \mathrm{~mm}$.

Table 1.2: System spatial resolution

\begin{tabular}{lll}
\hline \hline $\begin{array}{l}\text { Distance } \\
\text { from detector }(\mathrm{mm})\end{array}$ & $\begin{array}{l}\text { axial resolution } \\
(\mathrm{mm})\end{array}$ & $\begin{array}{l}\text { lateral resolution } \\
(\mathrm{mm})\end{array}$ \\
\hline 15 & 3.2 & 3.1 \\
60 & 3.9 & 4.4 \\
\hline \hline
\end{tabular}

In order to ascertain the maximum imaging depth, we resorted to simulations. We modeled the energy fluences at various depths in tissue mimicking materials for radiant exposure at the surface of $20 \mathrm{~mJ} \mathrm{~cm}^{-2}$ taking optical property values for breast tissue from the literature. The optical energy absorbed in spherical structures of various sizes and absorption coefficients can be calculated, leading to the calculation of the pressures generated. With this knowledge in combination with the MDP of the detector, it is possible to determine whether the pressure signal from a certain absorber at a certain depth is above the detection limit.

The MDP can be improved by signal averaging and using more detectors. Under these conditions, the maximum imaging depth for detecting a $2 \mathrm{~mm}$ sized sphere with a low contrast of $2 \mathrm{x}$ can reach $23 \mathrm{~mm}$. For inhomogeneities with higher contrast or 
with larger dimensions, the maximum imaging depth will be higher: e.g., a 5-mm sphere with a $7 x$ contrast will be detectable at a depth of $32 \mathrm{~mm}$. (See also [43] for an analysis of maximum theoretical imaging depths using PA.)

Experimentally the system maximum depth of imaging was assessed using inhomogeneities (M2) embedded in a phantom background (M1) to model a solid breast phantom [36,37]. M2 absorbing spheres of 2 and $5 \mathrm{~mm}$ diameter with 2x, 4x and 7x contrast were used at various depths; imaging was done with 100 detectors averaging over 100 pulses. In general, the results substantiated the simulations, with for example, an object of $2 \mathrm{~mm}$ diameter with a contrast of $7 \mathrm{x}$ being detectable at a depth of $32 \mathrm{~mm}$ (Table 1.4) [24-26, 28, 29, 48].

\begin{tabular}{lll} 
Table 1.3: System maximum imaging depth from simulation \\
\hline \hline $\begin{array}{ll}\text { M2 absorbing sphere } \\
\text { diameter }(\mathrm{mm})\end{array}$ & contrast & Maximum Imaging \\
& & Depth $(\mathrm{mm})$ \\
\hline \multirow{2}{*}{2} & $2 \mathrm{x}$ & 23 \\
& $4 \mathrm{x}$ & 26 \\
$7 \mathrm{x}$ & 29 \\
\hline 5 & $2 \mathrm{x}$ & 27 \\
$4 \mathrm{x}$ & 30 \\
$7 \mathrm{x}$ & 32 \\
\hline
\end{tabular}

\subsubsection{Reconstruction}

Image reconstruction is based on the delay and sum beamforming algorithm modified from [35]. The algorithm was tested on a phantom containing different shape absorbing bodies: integration of delay-and-sum signals provides satisfactory definition of the axial size of imaged objects. The reconstructed three-dimensional data is visualized using average intensity projections (AIPs) data; the volume of interest being divided into $0.5 \mathrm{~mm}$ voxels [29].

One detector element is receiving at each time, providing low hardware complexity for the system, and the synthetic aperture focusing is applied to the measured Ascans offline. The spherical wavefronts generated by a target absorber are detected at different times by different detector elements: different time delays have to be applied to the A-scans in order to have simultaneous detection of the signals at the elements. The sum of the delayed signals $\mathrm{O}$ at a certain voxel $\mathrm{V}$ is then the unique output 
associated to said point target in an appropriate time window of the signals [25]:

$$
\begin{aligned}
O^{V}(t) & =\frac{\sum_{i}^{V}\left[S_{i}(t) h\left(t+\tau+\delta_{i}^{V}\right)\right]_{|\max -\min |}}{\sum_{i} W_{i}^{V}} \\
& =\frac{\sum_{i}^{V}\left[S_{i}(t) h\left(t+\tau+\frac{\sqrt{x^{2}+y^{2}+z^{2}}}{v}\right)\right]_{|\max -\min |}}{\sum_{i} W_{i}^{V}} \\
& \text { with } h(t+\tau)= \begin{cases}1 & \text { if }|t| \leq \tau / 2 \\
0 & \text { otherwise }\end{cases}
\end{aligned}
$$

The focusing is performed by considering the distance from the imaging point to the receiving element of the transducer. The delay time $(\delta)$ to extract the proper signal value for summation is obtained by dividing this distance by the speed of sound. In Equation 1.3, $\mathrm{h}$ is a window function with width $\tau$; $\mathrm{S}$ the signal, and $\mathrm{W}$ the weighting factor depending on the element angular sensitivity; subscript index i stands for the detector element, and superscript V stands for the voxel.

\subsection{Clinical studies}

A pilot clinical study was conducted using PAM to detect breast cancer, comparing the obtained images with conventional imaging findings. A clinical protocol approved by Medical Ethics Committee of the Medisch Spectrum Twente hospital in Enschede, dictated the study; details are provided in $[26,28,48]$. In short, the patient arrives for the photoacoustic scan after having undergone the x-ray diagnostic scan and the ultrasonography.

A flow chart of the exam procedure is shown in Figure 1.3. At first the system is calibrated, by running self-tests and checks of ultrasound detector activation, data acquisition, file operations in writing data on the hard-disk, $\mathrm{x}-\mathrm{y}$ stage operation, and laser output. Homing the laser steps the scanning stage to the origin of the coordinates, where the head of a power and energy meter is positioned. The laser beam switches $\mathrm{ON}$ and the output is adjusted to the required output, after which it is switched OFF. A laser pointer is incorporated into the optics of the system as a pilot laser, in such a way that its visible beam is collinear with the NIR photoacoustic excitation beam. The scanning system is positioned so that this pilot beam impinges a landmark on the ultrasound detector face. This position is then registered; from this point onward the laser and detector coordinates are coupled. 


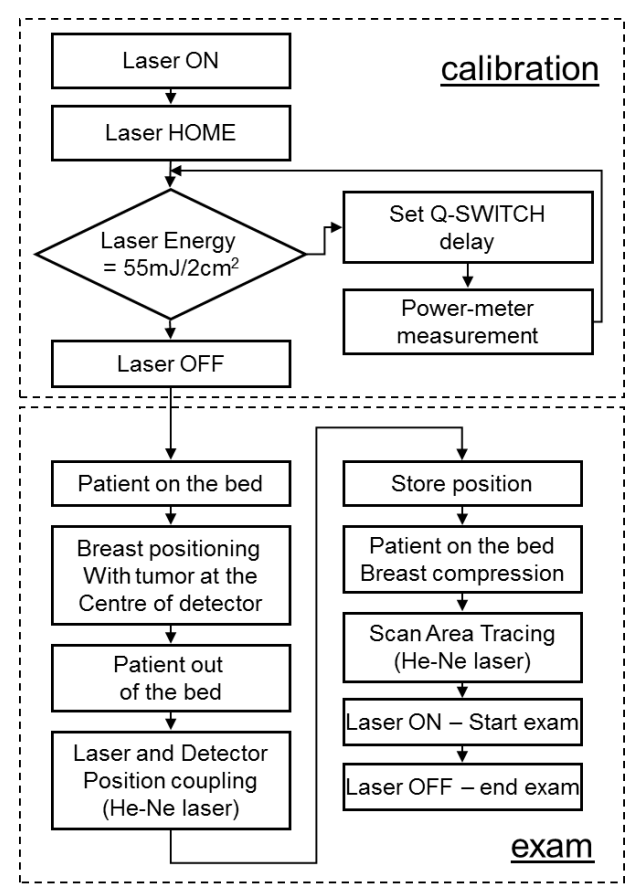

Figure 1.3: Flow chart of the photoacoustic examination preliminaries and procedure followed for the pilot patient studies.

The patient lies prone on the bed with her suspect or symptomatic breast through an aperture and hanging pendant. The $\mathrm{x}-\mathrm{y}$ scanning stage and ultrasound detector are together translated to have the ROI lying roughly in the center of the detector matrix. A scan area is chosen which is typically $55 \times 55 \mathrm{~mm}$, so that sufficient healthy tissue is included in the scan. The breast is then lightly compressed against the detector which has copious amounts of ultrasound coupling gel applied on its face. At this point to determine the relative position of the scan area with reference to the breast contour for the purposes of comparison with the x-ray images, a contour scan of the breast profile is made as follows. The operator controls the $x-y$ scanning stage with a joystick controller following the breast profile with the position of the pilot beam; the coordinates are registered and saved.

The measurement can then be started, and laser scanning and ultrasound detection takes place until the scan area is covered. The scan usually requires a duration of 20-30 minutes, after which the patient is moved to the biopsy theatre.

The acquired data is fed to the reconstruction algorithm. The reconstructed data set is normalized to a value of 255 and is visualized using maximum intensity 
projections (MIPs). The photoacoustic results from the pilot study were compared with findings of conventional techniques.

Thirteen patient measurements have been performed in the pilot study. Seven measurements were not applicable for further investigation: in three cases the tumor was too close to the chest wall and could not be reached in the scan area, in one case the scan area settings by operator were incorrect, and in the three last cases insufficient ultrasound gel between the breast and detector caused errors in measured signals.

Table 1.4: Patient studies

\begin{tabular}{|c|c|c|c|c|c|c|c|c|c|}
\hline \multirow{2}{*}{$\begin{array}{l}\text { Patient\# } \\
\text { (Case \#) } \\
\text { MST\# }\end{array}$} & \multirow{2}{*}{$\begin{array}{l}\text { Birth } \\
\text { data }\end{array}$} & \multirow{2}{*}{$\begin{array}{l}\text { Right or left } \\
\text { breast }\end{array}$} & \multirow{2}{*}{$\begin{array}{l}\text { Lesion } \\
\text { type }\end{array}$} & \multirow{2}{*}{$\begin{array}{l}\text { PAM } \\
\text { detection }\end{array}$} & \multicolumn{2}{|c|}{ Depth (mm) } & \multicolumn{3}{|c|}{ Diameter $(\mathrm{mm})$} \\
\hline & & & & & US & $\mathrm{PAM}^{\mathrm{a}}$ & US & Pathol. $^{\circ}$ & $\mathrm{PAM}^{\mathrm{b}}$ \\
\hline $\begin{array}{l}2(\text { case } 1) \\
06166651\end{array}$ & 1949 & Right & tumor & yes & 18 & 14 & 33 & 26 & 30 \\
\hline $\begin{array}{l}5 \text { (case } 2 \text { ) } \\
01574983\end{array}$ & 1956 & Right & tumor & yes & 16 & 14 & 17 & 32 & 35 \\
\hline $\begin{array}{l}8(\text { case } 3) \\
02352512\end{array}$ & 1962 & Left & cyst & NA & 17 & $\mathrm{ND}^{\mathrm{d}}$ & 22 & $\mathrm{NA}^{\mathrm{e}}$ & ND \\
\hline $\begin{array}{l}9 \text { (None) } \\
01430477\end{array}$ & 1955 & Right & tumor & no & 16 & ND & 13 & NA & ND \\
\hline $\begin{array}{l}\text { 10(None) } \\
04427321\end{array}$ & 1948 & Left & tumor & yes & 16 & 20 & 15 & NA & 13 \\
\hline $\begin{array}{l}13 \text { (None) } \\
02909912\end{array}$ & 1953 & Right & tumor & yes & 17 & 20 & 18 & 32 & 26 \\
\hline
\end{tabular}

a Depth taken as slice depth where absorber intensity is widest in the reconstructed data.

${ }^{\mathrm{b}}$ Diameter estimated as lateral major axis in reconstructed data.

${ }^{\mathrm{c}}$ Pathol. = Pathology

d $\mathrm{ND}=$ Not detected

e NA $=$ Not available

Basic results are consolidated in Table 1.4. In four of the five malignancies, the lesion was identified in the regions of higher absorption in the photoacoustic images. It is not known why a negative result was obtained in one case, but it is suspected that the scan region was not set corresponding to the ROI. There is also a photoacoustic scan of a ROI carrying a cyst where as expected no high intensity regions are observed. (See further Case 3.)

The depth of the lesion identified with PAM can be compared with the depth determined in the ultrasound image. It is also possible to extract depth information 
from the raw signals from individual elements of the detector when an absorbing structure can be identified. As an example the signal from one of the elements from Case 2 (patient 5) is given in Figure 1.4 where an absorbing structure can be identified at a distance of around $15 \mathrm{~mm}$ from the surface signal of the breast. However the signal-noise ratios from absorbers are not always so strong. It should be also noted that the quasi-exponential pressure decay resulting from in-depth distribution of optical absorption and heat release in the breast tissue is not visible because of the ultrasound detector frequency response (Figure 1.2(b)) where the lower cut frequency is about $450 \mathrm{kHz}$; the slow exponential decay is not reproduced and the appearance of the transient is sharp.

Comparison with ultrasound images was also done to assess the diameter of the lesions although it is difficult to define the diameter from the PAM images because the borders are not clear. For every patient the depth of the centre of the lesion and the diameter are given in Table IV for both techniques. Discrepancies between resulting values may be attributed to the mild compression in PAM which is not present in ultrasound.

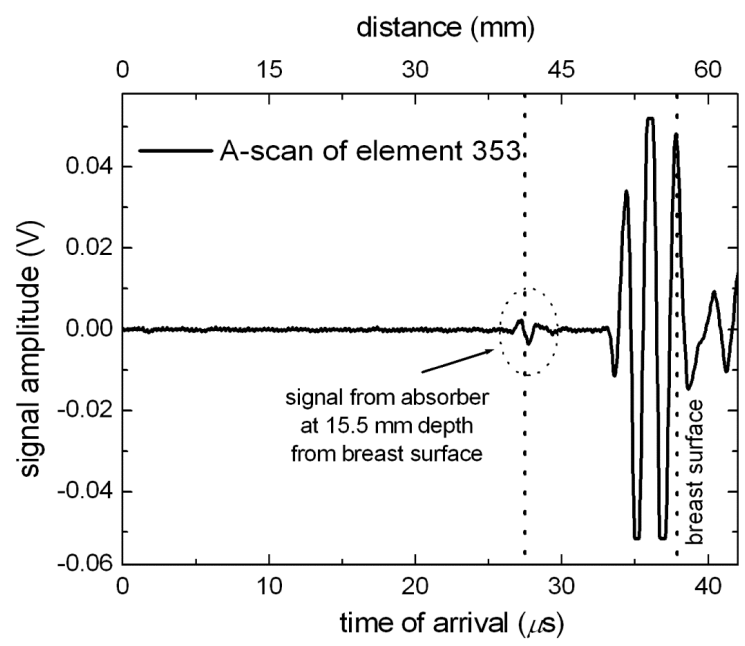

Figure 1.4: Signal trace of an element showing the photoacoustic signals arising the breast of Case 2. A large signal $\mathrm{f}$ produced at the breast surface; at a depth of approximately $15 \mathrm{~mm}$ from the illuminated surface the signal from the tumour is seen.

Three clinical results are discussed in detail: 


\subsubsection{Case 1}

This case has been described in [26]. The patient (Caucasian, 60 year old) was diagnosed with an infiltrating ductal carcinoma in the right breast. The x-ray mammogram is shown in Figure 1.5(a) and the ultrasound image in Figure 1.5(b). In the $\mathrm{x}$-ray image the ROI boundary where the photoacoustic scan took place is indicated with the dashed line. In Figure 1.5(c) the breast contour as observed under slight compression during the photoacoustic scan is depicted along with the scan region. Figure 1.5(d) is the MIP of the reconstructed photoacoustic data. The $\mathrm{x}$ and y axes of the image are respectively $46 \mathrm{x} 43 \mathrm{~mm}$ in dimension.
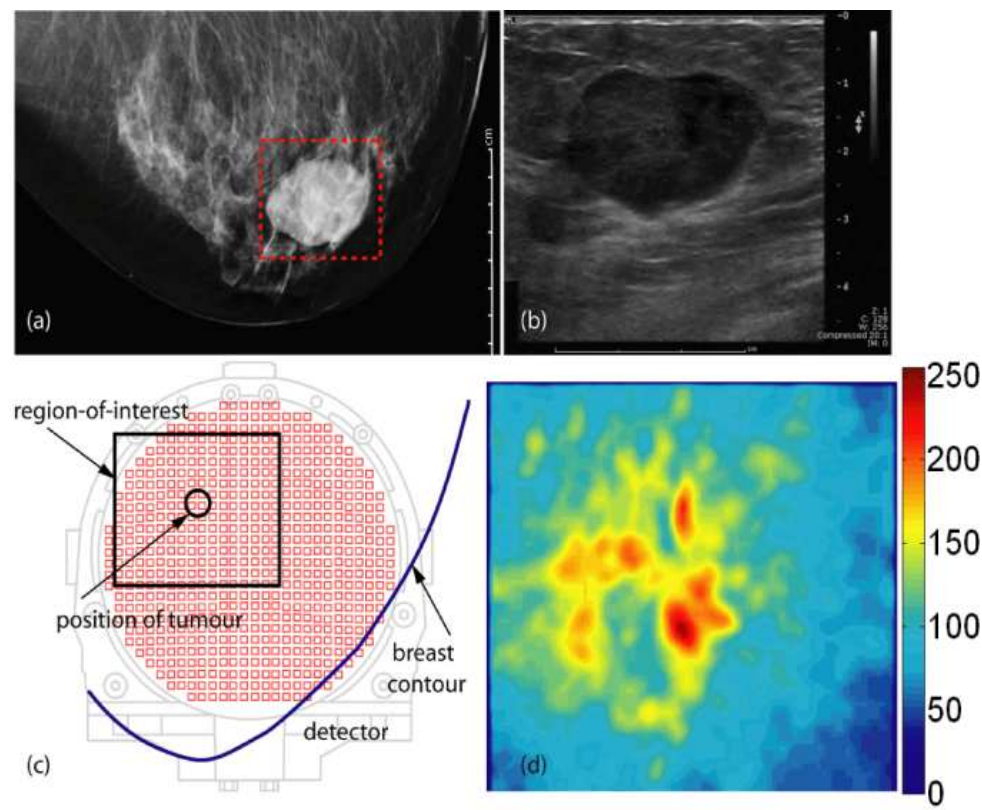

Figure 1.5: (a) Craniocaudal x-ray mammogram of Case 1 and (b) ultrasound image. Both show a large tumor mass with well defined margins. In the x-ray image, the supposed region-of-interest (ROI) is overlaid. (c) The contour of the breast under compression in the PAM, with a trace of the ROI and possible location of the tumor. This is shown with respect to the detector position. (d) Maximum Intensity Projection (MIP) of the three-dimensional photoacoustic reconstructed data. A ring-shaped region of high intensity probably marks the tumor rim where blood vessels are in abundance. The image size is $46 \times 43 \mathrm{~mm}$.

In both x-ray Figure 1.5(a), and ultrasound Figure 1.5(b) images, the tumor is visible as a mass. The tumor diameter measured in the x-ray image is $40 \mathrm{~mm}$ along 
the major axis, while the tumour is sized as $32.4 \mathrm{~mm}$ in the ultrasound image. In both cases the mass shows well defined boundaries and shapes. These are usually indications of a benign growth, but a closer look at the x-ray mammogram shows a cluster of microcalcifications which appear suspect. (For a detailed x-ray image see [26].) More than 10 heterogeneous and punctuate microcalcifications are grouped in the left side of the tumor. Some brighter spots are also visible in the ultrasound image, perhaps due to the microcalcifications. The radiologist recommended that a biopsy be taken of the tumour. In the MIP image of the three-dimensional photoacoustic reconstructed data (Figure 1.5(d)) a ring-shaped area of higher intensity with a diameter around $28 \mathrm{~mm}$ is found. Examination of the individual slices (see Ref. [26]) shows that this absorbing inhomogeneity extends from around 7 to $21 \mathrm{~mm}$ depth from the surface of the breast. A contrast in the PA image (CNR, obtained with the ratio of the maximum value in the image to the background level) of 1.88 was determined by inspection of the slice where the intensity area was largest. We attribute this contrast to the presence of tumour vascularization. The ring marks the periphery of the cancer growth region where substantial angiogenesis is expected, with the central region possessing lower perfusion.

\subsubsection{Case 2}

This case has been described in Ref. [26]. The patient (Caucasian, 50 year old) was diagnosed with an infiltrating ductal carcinoma. X-ray mammography in Figure 1.6(a) did not reveal a mass, rather an area of architectural distortion with speculation. A cluster of microcalcifications within the region was also present in a zoomed in image (not shown here). The ROI region where the photoacoustic scan took place is indicated with dashed line. The ultrasonogram (Figure 1.6(b)) reveals a solid mass with poor echogenicity and with irregular margins. The tumor has a width of $17 \mathrm{~mm}$ and a depth of $16 \mathrm{~mm}$ in the ultrasound image. The lesion is highly suspicious for malignancy and a core biopsy was recommended. The scan area (53 x $40 \mathrm{~mm})$ with respect to the breast contour and the detector is shown in Figure 1.6(c).

A volume isosurface rendering of the three-dimensional photoacoustic data is presented in Figure 1.6(d) where high intensity regions are depicted. These regions are due to localized hemoglobin distributions arising from the vascularization that is characteristic to malignity. A CNR of 1.45 was determined by examination of the individual slice data where this intensity area was largest. The size of the lesion was determined to be about $35 \mathrm{~mm}$ in this slice, while post-surgical histology exam 


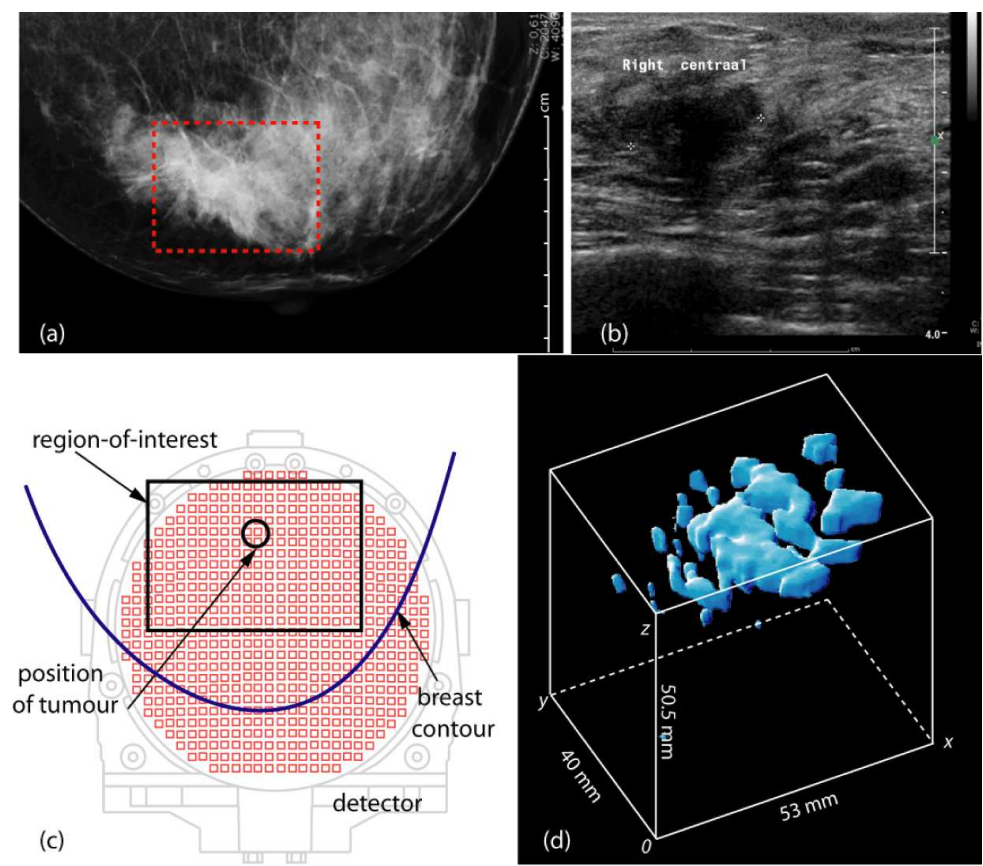

Figure 1.6: (a) Craniocaudal x-ray mammogram of Case 2 and (b) ultrasound image. No mass is seen in the x-ray, but ultrasound shows a suspect tumor with poorly defined margins. (c) The contour of the breast under compression in the PAM, with a trace of the ROI and possible location of the tumor. This is shown with respect to the detector position. (d) Isosurface rendering of the three-dimensional photoacoustic reconstructed data. The image size is $40 \times 53 \mathrm{~mm}$.

returned a $32 \mathrm{~mm}$ carcinoma. The depth of the lesion, identified with the PAM is comparable with the depth determined in the ultrasound image: $16 \mathrm{~mm}$ in the ultrasound image and $14 \mathrm{~mm}$ in the photoacoustic one.

\subsubsection{Case 3}

This patient (Caucasian, 49 year old) was diagnosed with a cyst. The x-ray mammogram (not shown), revealed a mass visible in the upper left corner of the image. The mass was round with clear and smooth border and highly likely to be a cyst. This was confirmed with ultrasonography. The ultrasound image (Figure 1.7(a)) shows the lesion as completely anechoic and elliptic shaped. Further, there is clear posterior echo enhancement. All these are signature features of a cyst, with a size of $22.3 \mathrm{~mm}$ from the sonogram.

PAM was set to scan the ROI in an area of $34 \times 46.5 \mathrm{~mm}$. The reconstructed data 
is shown in a MIP in isometric view in Figure 1.7(b). High intensities are seen in two areas at the upper left corners of the scan area. These are at depths of approximately $33 \mathrm{~mm}$ and $45 \mathrm{~mm}$ from the illuminated surface. Significantly in the rest of the volume no heterogeneities are seen. It is unclear what the nature of the absorbing structures lying deep in the breast are. The high intensities suggest absorption by large blood vessels but in the absence of any verification, this remains speculation. However, if we concentrate on the region where the cyst is located (15 mm deep) we do not observe any distinguishable features right down to depths of $30 \mathrm{~mm}$. This is to be expected as enhanced vascularisation is not associated with a cyst. The most important conclusion that we can make from this measurement, is that the cyst does not produce discernible photoacoustic signals which is to be expected since this benign abnormality is not characterized by vascularisation.
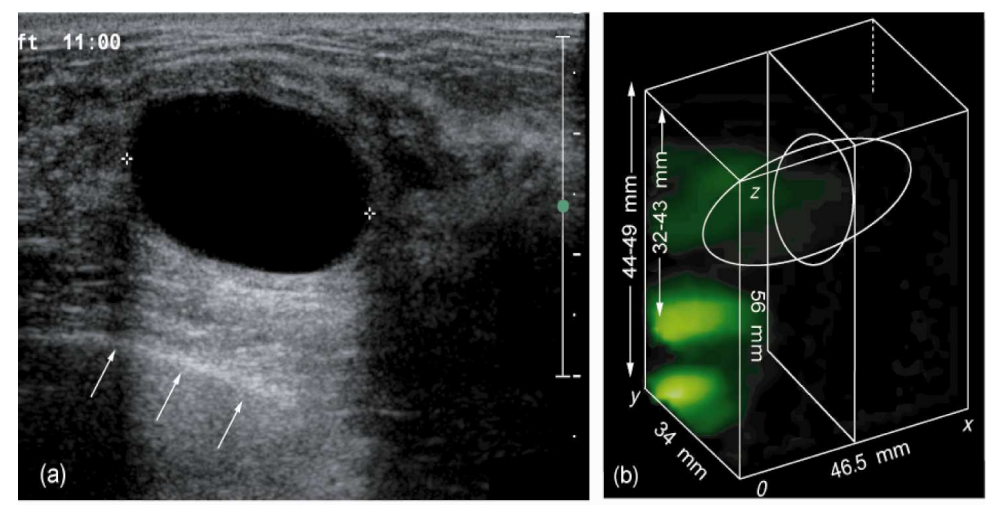

Figure 1.7: (a) Ultrasound image of an abnormality in the breast of Case 3. At a depth of approximately $15 \mathrm{~mm}$ is seen a $23 \mathrm{~mm}$ diameter cyst. This can be recognized by the anechoic properties of the object with posterior enhancement. (b) Isometric view of the MIP of reconstructed photoacoustic data from the ROI carrying the cyst: two ellipses are marked in the figure where the cyst is expected to be according to the relative ultrasound image. No absorbers are seen where the cyst is expected, down to a depth of $30 \mathrm{~mm}$. A cyst does not possess enhanced vascularization and will remain "photoacoustically silent." It is not known why intense signals are present at higher depths roughly $33 \mathrm{~mm}$ and $45 \mathrm{~mm}$. 


\subsection{Future work: speed of sound tomography with PA}

The instrument will be upgraded to scan a larger area of the breast, preferably the whole breast. Whole breast imaging is a condition sine qua non if screening applications are being pursued. Further, the system will be modified to have parallel acquisition from a number of elements thereby reducing measurement times which will result in better images as the possibility of motion artifacts will be reduced, but will also see better acceptance in the clinic.

A computed tomography approach (CT) is being considered with a circular detector positioned around the whole breast; light admitted from all directions. The multiple views of the object through a number of independent realizations of the same cross-section, will enhance the resolution and contrast of the image. Further, multiple angle views inherent to CT will avoid the appearance of shadowing due to strong absorbers in the images as could be the case with one or a few projections. The main disadvantages of this approach is that a large water filled tank will be required for accommodating the breast, and the complexities that are associated with the circular detector array and light delivery approach. CT has been used by a number of photoacoustic research groups resembling configurations used for ultrasound CT [44]. In [45] a $780 \mathrm{~nm}$ laser source and a $5 \mathrm{MHz}$ circular array detector are used for small animal imaging. Ref. [23] reports a CT configuration for breast imaging with a similar layout for the ultrasound transducer. An alternative option to this completely enclosing detector is described in [22] which is a modified version of the past TCT scanner [46] and using a $434 \mathrm{MHz}$ radio frequency source and a $1 \mathrm{MHz}$ tri-planar transducer array for breast imaging.

We will also implement in the new CT breast imager, a recent method which permits imaging acoustic attenuation (AA) and speed-of-sound (SOS) in a photoacoustic imager $[47,48]$. These parameters SOS and AA have diagnostic value in identifying malignant tissues. As reported in $[49,50]$ malignancies have higher SOS with respect to healthy surrounding tissues. Further, high AA values are associated with malignancies regardless of the corresponding SOS. As described in $[47,48]$ a method is available that permits the measurement of these parameters simultaneous to the acquisition of conventional photoacoustic data during a CT measurement. Thus, maps of the ultrasound transmission parameters SOS and AA can be reconstructed along with accompanying photoacoustic images providing more information about the properties of the investigated tissue. 

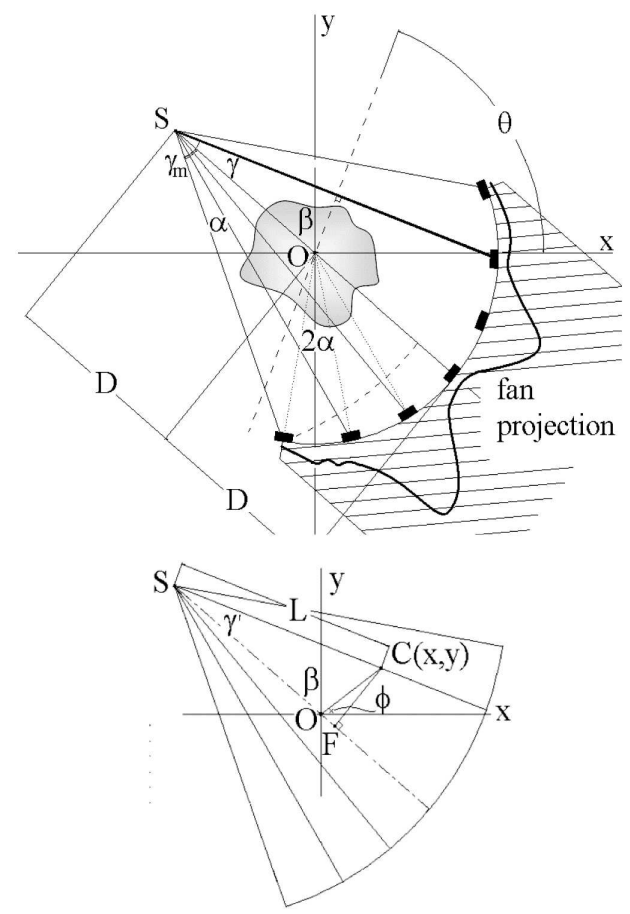

Figure 1.8: Schematic of the method for simultaneous imaging of speed-of-sound, acoustic attenuation and photoacoustics in a CT geometry. At S a carbon fibre is present in acoustic contact with the object at the origin and the curvilinear detector array at the far-end. Ultrasound is emitted from the carbon fibre when it is illuminated with pulsed light, which interacts with the object before being detected by the detector. A fan-beam projection is obtained. The data from such a projection is analyzed both in amplitude and time of arrival compared with a homogeneous reference measurement. Multiple projections around the object allow the reconstruction of the ultrasound transmission parameters.

In this, an ultrasound curvilinear detector and a laser source are arranged in a CT configuration, where both are fixed in a water tank and in such a way as to have the centre of curvature of the array at the centre of the imaging plane. A thin absorbing element (carbon fiber) is placed in front of the laser source. The absorber produces a short acoustic pulse by the photoacoustic effect and can be considered as a source of ultrasound. The ultrasound wave passes through the object, is modulated by the object acoustic properties, and is measured at the far-end at the detector $[47,48,51,52]$. Projections are obtained from all around the object. The sinograms of the time-offlight (TOF) values can be obtained in a similar manner as in ultrasound CT by estimating the arrival time values for each sensor element at each angle separately. 
This measurement traces line integrals between the carbon fiber and each element of the array at an angle. These form a fan-beam as depicted in Figure 1.8. If $\beta$ is the angle that the source makes with the y reference axis and $\gamma$ the location of the generic ray within the fan (Figure 1.8), once a set of projections have been acquired, the reconstruction of the physical property from which said projection has originated, is expressed as follows [53]:

$$
f(r, \phi)=\int_{0}^{2 \pi} \frac{1}{L^{2}} Q_{\beta}(\gamma) d \beta
$$

under the assumptions of evenly distributed beam rays and equally spaced detector elements, Equation 1.5 provides the Radon reconstruction, where $\mathrm{Q}_{\beta}$ is effectively the filtered backprojection measured from one angle.

Details of this approach are discussed in $[47,48,51,52]$ where the concepts have been tested on phantoms with speed-of-sound inhomogeneities. Further the same analysis is applicable to measurement of acoustic attenuation as well.

\subsection{Conclusions}

Promising results were obtained with the detection of malignancies in the breast using the photoacoustic technique. The principal goal of this pilot study about the possibility to detect breast tumors with this setup has been answered, not only in the heightened intensity in the images but also in distinctive transients in some individual signal traces of each element. Further, the image of the ROI carrying a cyst represents a successful negative control experiment, as it were, where the lack of neo-vascularization results in the absence of absorbing inhomogeneities in the image.

The main disadvantage of the system in its present state is the limited angular view problem compared to 360 degree TCT [22] and 120 degree LOIS [21]. Conversely, an advantage is the easier comparison between PAM images and x-ray images because of the use of a planar compressed geometry. The system also suffers from long measurement times, upto 30 minutes, since the ultrasound detector works with one element active at each time step. We intend to address these issues in the immediate future while resuming clinical studies with the present embodiment of the instrument. 


\section{Bibliography}

[1] W. R. Hendee, and E. R. Ritenour, "Medical Imaging Physics," New York: John Wiley \& Sons, ch.26, (2002).

[2] S. J. Nass, I. C. Henderson, and J. C. Lashof, "Mammography and beyond: developing technologies for the early detection of breast cancer," Washington DC: National Academy Press, (2001).

[3] S.B. Colak, M. B. van der Mark, G. W. 't Hooft, J. H. Hoogenraad, E. S. van der Linden, and F. A. Kuijpers, "Clinical optical tomography and NIR spectroscopy for breast cancer detection," IEEE J. Select. Top. Quantum. Electron. 5, 11431158, (1999).

[4] B. W. Pogue, S. P. Poplack, T. O. McBride, W. A. Wells, K. S. Osterman, U. L. Osterberg, and K. D. Paulsen, "Quantitative haemoglobin tomography with diffuse near-infrared spectroscopy: pilot results in the breast," Radiology 218, 26-266, (2001).

[5] D. Grosenick, K. T. Moesta, H. Wabnitz, J. Mucke, C. Stroszczynski, R. Macdonald, P. M. Schlag, and H. Rinneberg, "Time-domain optical mammography: initial clinical results on detection and characterization of breast tumors," Appl. Opt. 42, 3170-3186, (2003).

[6] E. L. Heffer, and S. Fantini, "Quantitative oximetry of breast tumors: a near infrared method that identifies two optimal wavelengths for each tumor," Appl. Opt. 41, 3827-3839, (2002).

[7] B. J. Tromberg, N. Shah, R. Lanning, A. Cerrusi, J. Espinoza, T. Pham, L. Svaasand, and J. Butler, "Non invasive in vivo characterization of breast tumors using photon migration spectroscopy," Neoplasia 2, 26-40, (2000).

[8] K. Suzuki, Y. Yamashita, K. Otha, M. Kaneko, M. Yoshida, and B. Chance, "Quantitative measurement of optical parameters in normal breasts using time- 
resolved spectroscopy: in vivo results of 30 Japanese women," J. Biomed. Opt. 1, 330-334, (1996).

[9] T. O. McBride, B. W. Pogue, S. Jiang, U. L. Orserberg, K. D. Paulsen, and S. P. Poplack, "Initial studies of in vivo absorbing and scattering heterogeneity in near-infrared tomographic breast imaging," Opt. Lett. 26, 822-824, (2001).

[10] J. Folkman, "Tumor angiogenesis," in Cancer Medicine, 5th ed., J F Holland, Ed. Hamilton, ON: B. C. Decker Inc, pp. 132-152, ch. 9, (2000).

[11] R. L. P. Van Veen, A. Amelink, M. Menke-Pluymers, C. Van der Pol, and H. J. C. M. Sterenborg, "Optical biopsy of breast tissue using differential path-length spectroscopy," Phys. Med. Biol. 50, 2573-2581, (2005).

[12] N. Shah, A. Cerussi, C. Eker, J. Espinoza, J. Butler, J. Fishkin, R. Hornung, and B. Tromberg, "Non invasive functional optical spectroscopy of human breast tissue," Proc. Nat. Acad. Sci. USA, 98, 4420-4425, (2001).

[13] V. V. Tuchin, "Tissue Optics: Light Scattering Methods and Instrumentation for Medical Diagnosis," in Tutorial texts in optical engineering, vol. TT38, D. C. O'Shea, Ed. Bellingham, WA: SPIE Press, p. 42, (2000).

[14] R. A. Kruger, "Photoacoustic ultrasound," Med. Phys. 21, 127-131, (1994).

[15] A. A. Oraevsky, S. L. Jacques, R. O. Esenaliev, and F. K. Tittel, "Time-resolved optoacoustic imaging in layered biological tissues," in Advances in Optical Imaging and Photon Migration, vol. 21, R. R. Alfano, Ed. San Diego, CA: Academic, pp. 161-165, (1994).

[16] L. V. Wang, "Prospects of photoacoustic tomography," Med. Phys. 35, 57585767, (2008).

[17] R. A. Kruger, W. L. Jr Kiser, D. R. Reinecke, and G. A. Kruger, "Application of thermoacoustic computed tomography to breast imaging," Proc. SPIE 3659, 426-430, (1999).

[18] R. A. Kruger, W. L. Jr Kiser, K. D. Miller, and H. E. Reynolds, "Thermoacoustic CT scanner for breast imaging: design considerations," Proc. SPIE 3982, 354$359,(2000)$. 
[19] A. A. Oraevsky, and A. A. Karabutov, "Optoacoustic tomography," ,in Biomedical photonics handbook, T. Vo-Dinh, Ed. Boca Raton, FL: CRC Press, pp. 1-31, (2003).

[20] S. A. Ermilov, T. Khamapirad, A. Conjusteau, M. H. Leonard, R. Lacewell, K. Mehta, T. Miller, and A. A Oraevsky, "Laser optoacoustic imaging system for detection of breast cancer," J. Biomed. Opt. 14, 024007-024014, (2009).

[21] A. A Oraevsky, "Optoacoustic tomography of the breast," ,in Photoacoustic imaging and spectroscopy, L. V. Wang, Ed. Boca Raton, FL: CRC Press, pp. 411-429, (2009).

[22] R. A. Kruger, "Thermoacoustic computed tomography of the breast," in Photoacoustic imaging and spectroscopy, L. V. Wang, Ed. Boca Raton, FL: CRC Press, pp. 331-338, (2009).

[23] M. Pramanik, G. Ku, C. Li, and L. V. Wang, "Design and evaluation of a novel breast cancer detection system combining both thermoacoustic (TA) and photoacoustic (PA) tomography," Med. Phys. 35, 2218-2223, (2008).

[24] S. Manohar, A. Kharine, J. C. G. van Hespen, W. Steenbergen, and T. G. Van Leeuwen, "Photoacoustic mammography laboratory prototype: imaging of breast tissue phantoms," J. Biomed. Opt. 9, 1172-1181, (2004).

[25] S. Manohar, A. Kharine, J. C. G. van Hespen, W. Steenbergen, and T. G. Van Leeuwen, "The Twente Photoacoustic Mammoscope: system overview and performance," Phys. Med. Biol. 50, 2543-2557, (2005).

[26] S. Manohar, S. E. Vaartjes, J. C. G. Van Hespen, J. M. Klaase, F. M. Van den Engh, W. Steenbergen, and T. G. Van Leeuwen, "Initial results of in vivo noninvasive cancer imaging in the human breast using near-infrared photoacoustics," Opt. Express 15, 12277-12285, (2007).

[27] S. Manohar, S. E. Vaartjes, J. C. G. Van Hespen, J. M. Klaase, F. M. Van den Engh, W. Steenbergen, and T. G. Van Leeuwen, "Region-of-interest breast images with the Twente photoacoustic mammoscope (PAM)," SPIE Proc. 6437, 643702, (2007).

[28] S. Manohar, A. Kharin, C. De Vos, J. C. G. Van Hespen, W. Steenbergen, and T. G. Van Leeuwen, "The Twente photoacoustic mammoscope: toward clinical 
trials," in Emerging Technologies in Breast Imaging and Mammography, J. S. Suri, R. M. Rangayyan \& S. Laxminarayan, Ed. Los Angeles, CA: American Scientific Publishers, pp. 243-274, (2008).

[29] S. Manohar, T. G. van Leeuwen, J. M. Klaase, F. M. Van den Engh, W. Steenbergen, "Photoacoustic mammography with a flat detection geometry," in Photoacoustic imaging and Spectroscopy, L. V. Wang, Ed. Boca Raton, FL: CRC Press, pp. 431-442, (2009).

[30] M. W. Sigrist, and F. K. Kneubühl, "Laser-generated stress waves in liquids," J. Acoust. Soc. Am. 64, 1652-1663, (1978).

[31] G. J. Diebold, and T. Sun, "Properties of photoacoustic waves in one, two and three dimensions," Acustica 80, 339-351, (1994).

[32] C. G. A. Hoelen, and F. F. M. de Mul, "A new theoretical approach to photoacoustic signal generation," J. Acoust. Soc. Am. 106, 695-706, (1999).

[33] M. S. Patterson, B. C. Wilson, and D. R. Wyman, "The propagation of optical radiation in tissue. II: optical properties of tissues and resulting fluence distributions," Lasers in Medical Science 6, 379-390, (1991).

[34] H. Brecht, R. Su, M. Fronheiser, S. A. Ermilov, A. Conjusteau, A. Liopo, M. Motamedi, and A. A. Oraevsky, "Optoacoustic 3D whole-body tomography: experiments in nude mice," SPIE Proc. 7177, 71770E, (2009).

[35] C. G. A. Hoelen, A. Dekker, and F. F. De Mul, "Image reconstruction for photoacoustic scanning of tissue structures," Appl. Opt. 39, 5872-5883, (2000).

[36] H. J. Van Staveren, C. J. M. Moes, J. Van Marle, S. A. Prahl, and M. J. C. Van Gemert, "light scattering in intralipid-10\% in the wavelngth range 400-1100 nm," Appl. Opt. 30, 4507-4514, (1991).

[37] A. Kharine, S. Manohar, R. Seeton, R. G. M. Kolkman, R. A. Bolt, W. Steenbergen, and F. F. M. de Mul, "Poly(vinyl alcohol) gels for use as tissue phantoms in photoacoustic mammography," Phys. Med. Biol. 48, 357-370, (2003).

[38] S. Manohar, A. Kharine, W. Steenbergen, and T. G. van Leeuwen, "Threedimensional photoacoustic imaging of breast tissue phantoms," SPIE Proc. $\mathbf{5 4 8 6 ,}$ 247-259, (2004). 
[39] A. A. Oraevsky, V. G. Andreev, A. A. Karabutov, S. V. Solomatin, E. V. Savateeva, R. D. Fleming, Z. Gatalica, and H. Singh, "Laser optoacoustic imaging of breast cancer in vivo," SPIE Proc. 4256, 81-94, (2001).

[40] M. Xu, and L. V. Wang, "Analytical explanation of spatial resolution related to bandwidth and detector aperture size in thermoacoustic or photoacoustic reconstruction," Phys. Rev. E. 67, 1-15, (2003).

[41] T. D. Khokhlova, I. M. Pelianov, and A. A. Karabutov, "Optoacoustic tomography utilizing focused transducers: The resolution study," Appl. Phys. Lett. 92, 024105, (2008).

[42] V. G. Andreev, A. A. Karaboutov, and A. A. Oraevsky, "Detection of ultrawideband ultrasound pulses in optoacoustic tomography," IEEE Trans. Ultrason. Ferroelectr. Freq. Control 50, 1583-1590, (2003).

[43] T. D. Khokhlova, I. M. Pelianov, V. V. Kozhushko, A. N. Zharinov, V. S. Solomatin, and A. A. Karabutov, "Optoacoustic imaging of absorbing objects in a turbid medium: ultimate sensitivity and application to breast cancer diagnostics," Appl. Opt. 46, 262-272, (2007).

[44] N. Duric, P. Littrup, A. Babkin, D. Chambers, S. Azevedo, A. Kalinin, R. Pevzner, M. Tokarev, E. Holsapple, O. Rama, and R. Duncan, "Development of ultrasound tomography for breast imaging: Technical assessment," Med. Phys. 32, 1375-1386, (2005).

[45] J. Gamelin, A. Aguirre, A. Maurudis, F. Huang, D. Castillo, L. Wang, and Q. Zhu, "Design and characterization of an array-based photoacoustic tomographic system for small animal imaging," in Photoacoustic imaging and spectroscopy, L. V. Wang, Ed. Boca Raton, FL: CRC Press, 309-328, (2009).

[46] R. A. Kruger, K. D. Miller, H. E. Reynolds, W. L Kiser, D. R. Reinecke, and G. A. Kruger, "Breast cancer in vivo: contrast enhancement with thermoacoustic CT at 434 MHz- Feasibility study," Radiology 216, 279-283, (2000).

[47] R. G. H. Willemink, S. Manohar, C. H. Slump, F. van der Heijden, and T. G. van Leeuwen, "Acoustic property measurements in a photoacoustic imager," SPIE Proc. 6631, 663109, (2007). 
[48] S. Manohar, R. G. H. Willemink, F. van der Heijden,C. H. Slump, and T. G. van Leeuwen, "Concomitant speed-of-sound tomography in photoacoustic imaging," Appl. Phys. Lett. 91, 131911, (2007).

[49] C. Li, N. Duric, P. Littrup, and L. Huang, "In vivo breast soundspeed imaging with ultrasound tomography, "Concomitant speed-of-sound tomography in photoacoustic imaging," Ultras. Med. Biol. 91, in press, (2009).(doi:10.1016/j.ultrasmedbio.2009.05.011).

[50] J. S. Schreiman, J. J. Gisvold, J. F. Greenleaf, and R. C. Bahn, "Ultrasound computed tomography of the breast," Radiology 150, 523-530, (1984).

[51] R. G. H. Willemink, S. Manohar, Y. Purwar, C. H. Slump, F. van der Heijden, and T. G. van Leeuwen, "Imaging of acoustic attenuation and speed of sound maps using photoacoustic measurements," SPIE Proc. 6920, 692013, (2008).

[52] R. G. H. Willemink, S. Manohar, J. Jithin, C. H. Slump, F. van der Heijden, and T. G. van Leeuwen, "Simultaneous imaging of ultrasound attenuation, speed of sound, and optical absorption in a photoacoustic setup," SPIE Proc. 7265, 72650J, (2009).

[53] A. C. Kak, and M. Slaney, "Principle of computerized tomographic imaging," New York: IEEE Press, ch. 3, (1988). 


\section{Chapter 2}

\section{Poly(vinyl alcohol) gels as photoacoustic breast phantoms revisited}

2 Abstract

A popular phantom in photoacoustic imaging is poly(vinyl alcohol) (PVA) hydrogel fabricated by freezing and thawing (F-T) aqueous solutions of PVA. The material possesses acoustic and optical properties similar to those of tissue. Earlier work characterized PVA gels in small test specimens where temperature distributions during F-T are relatively homogeneous. In this work, in breast-sized samples we observed substantial temperature differences between the shallow regions and the interior during the F-T procedure. We investigated whether spatial variations were also present in the acoustic and optical properties. The speed of sound, acoustic attenuation and optical reduced scattering coefficients were measured on specimens sampled at various locations in a large phantom. In general, the properties matched values quoted for breast tissue. But while acoustic properties were relatively homogeneous, the reduced scattering was substantially different at the surface compared with the interior. We correlated these variations with gel microstructure inspected using scanning electron microscopy. Interestingly, the phantom's reduced scattering spatial distribution matches the optical properties of the standard two-layer breast model used in x-ray

\footnotetext{
${ }^{2}$ Part of this chapter has been published as: W. Xia, D. Piras, M. Heijblom, W. Steenbergen, T. G. van Leeuwen and S. Manohar, "Poly(vinyl alcohol) gels as photoacoustic breast phantoms revisited," J. Biomed. Opt. 16(7), (2011), 075002. Reproduced with permission.
} 
dosimetry. We conclude that large PVA samples prepared using the standard recipe make excellent breast tissue phantoms.

\subsection{Introduction}

Tissue mimicking phantoms are important for the evaluation of the performance characteristics of an imaging system, such as resolution, sensitivity and imaging depth [1]. Most phantoms are designed for the testing of one particular imaging modality. Photoacoustics is an intrinsically hybrid approach which is characterized by light and sound propagation; applied pulsed light excites ultrasound from absorbing structures, such as blood vessels and angiogenic tumor masses, which can be detected. The advantages and various applications of photoacoustic imaging have been reviewed by Wang [2]. One of the important applications is photoacoustic breast imaging, an area of intense research interest [3-6], as a promising alternative technology to detect angiogenic markers of breast cancer based on hemoglobin absorption. Phantoms for photoacoustic (PA) breast imaging have to be designed to accomplish both optical and acoustic properties of soft tissues.

Poly(vinyl alcohol) (PVA) gels were introduced as photoacoustic phantoms by Kharine et al $[7,8]$ and used by several groups for photoacoustic imaging $[9,10]$ and optical elastography [11]. PVA gels are formed by the physical crosslinking of hydroxyl groups by hydrogen bonding in the process of freezing and thawing (F-T). The F-T process promotes the formation of crystallites in an amorphous matrix [12]. The number, size and stability of crystallites are increased with an increasing number of F-T cycles by which the mechanical strength of the gel increases $[12,13]$. Therefore, no additional chemical agents are required for gelation. Such gels were used earlier to mimic soft tissues in ultrasound [14,15] and magnetic resonance imaging [16,17]. Kharine et al [7] found that repeated F-T also led to increased light scattering in the gel. During freezing, the gel is separated into an ice phase and a polymer phase. A significant volume expansion takes place in the formation of the ice phase, leaving pores during thawing which causes refractive index fluctuations, and hence optical scattering. Kharine et al [7] optimized the number of F-T cycles and thereby controlled the optical characteristics of the resulting gel to match values quoted for breast tissue. Coupled with the favorable acoustic properties, the gels were found to be excellent photoacoustic phantoms [18].

Various properties of the hydrogels depend on the specifications of the F-T processing that they undergo, such as the number of F-T cycles, the durations of F-T, 
the rates of $\mathrm{F}-\mathrm{T}$ and the actual temperatures attained $[19,20]$. While Kharine et al [7] studied only the effect of number of F-T cycles on the phantom properties, the influence of the other variables is not known. The actual temperature achieved and the rates of F-T experienced depend on the sizes of the gel produced and for the large phantoms required for breast imaging, these are expected to be spatially non-uniform. These spatial temperature responses could have an effect on the relevant phantom properties, which if not taken into account could cause inaccurate estimation of performances of the PA imaging system.

In this work, we investigate the spatial distribution of phantom properties in a large PVA breast sample. We study the distribution of reduced scattering coefficient $\left(\mu_{s}^{\prime}\right)$, speed of sound (SOS) and acoustic attenuation (AA) in the phantom. These properties are discussed in the context of spatial temperature variations monitored during the F-T process and correlated with the microstructure examined with scanning electron microscopy (SEM). We then discuss the suitability of the breast samples as phantoms for photoacoustic mammography.

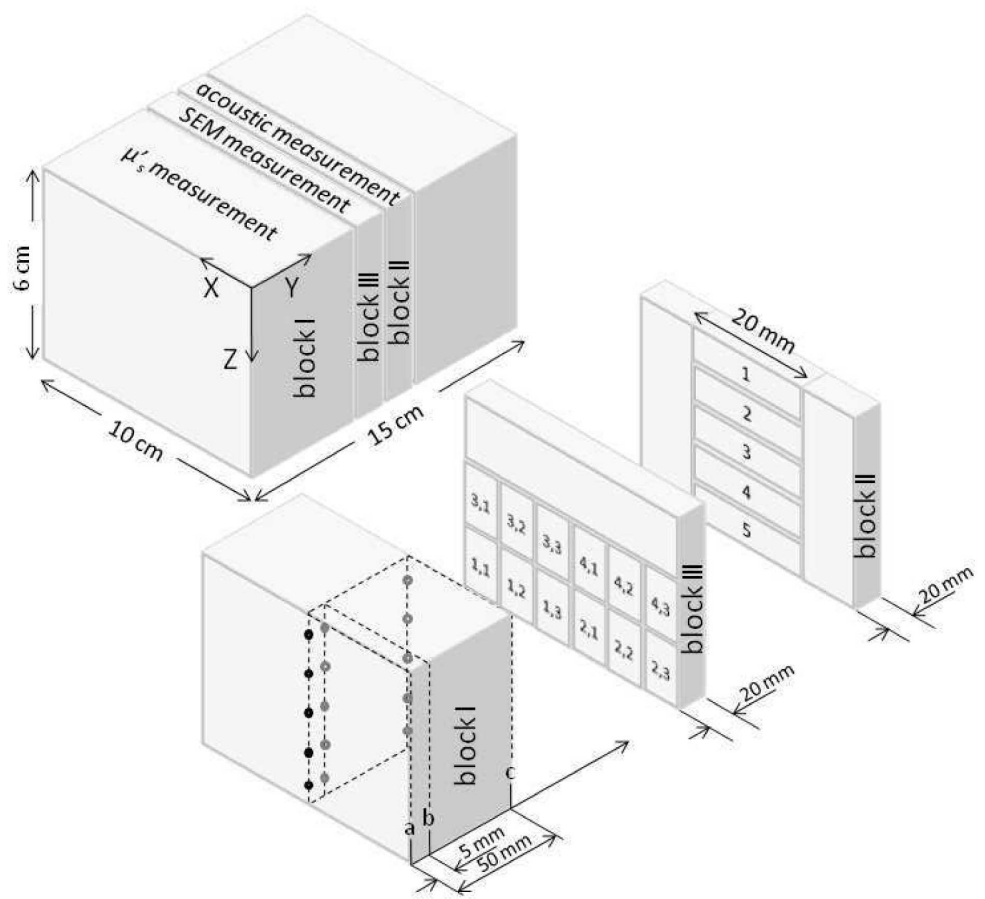

Figure 2.1: Schematic of poly(vinyl alcohol) gel prepared for speed of sound, acoustic attenuation, optical reduced scattering coefficient and microstructure measurements. 


\subsection{Materials and Methods}

\subsubsection{PVA phantom preparation}

The recipe for making PVA gel based phantoms has been described in Kharine et al [7] and Manohar et al [18]; here we briefly summarize the procedure. PVA with hydrolysis degree greater than $99 \%$ and an average molecular weight of 85000-140000 (Sigma-Aldrich, catalog nr 36 314-6) is used. An aqueous solution of $20 \mathrm{wt} \%$ PVA is obtained by dissolving PVA powder in demineralized water at $95{ }^{\circ} \mathrm{C}$ with continuous gentle stirring for $2 \mathrm{~h}$. After it is allowed to stand for a few hours to let air bubbles migrate to the surface, the solution is cast in a Perspex mould and kept for $12 \mathrm{~h}$ at -20 ${ }^{\circ} \mathrm{C}$ in a refrigerator and subsequently for $12 \mathrm{~h}$ at room temperature. This constitutes one F-T cycle. The final sample is prepared undergoing 4 F-T cycles according to Kharine et al [7].

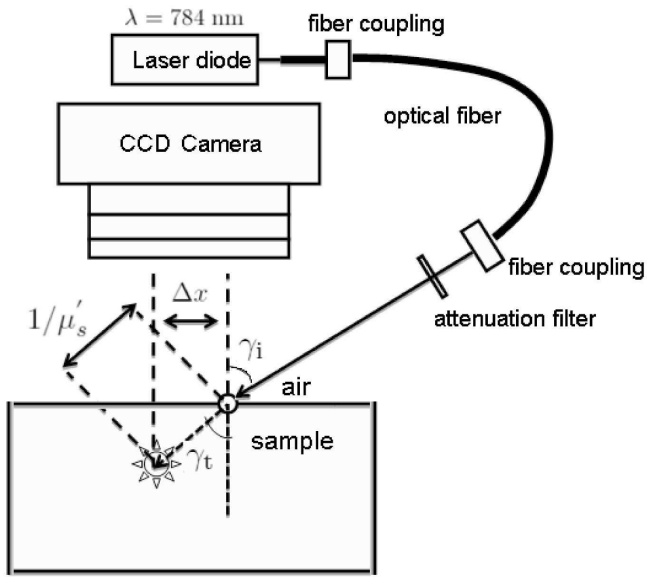

a)
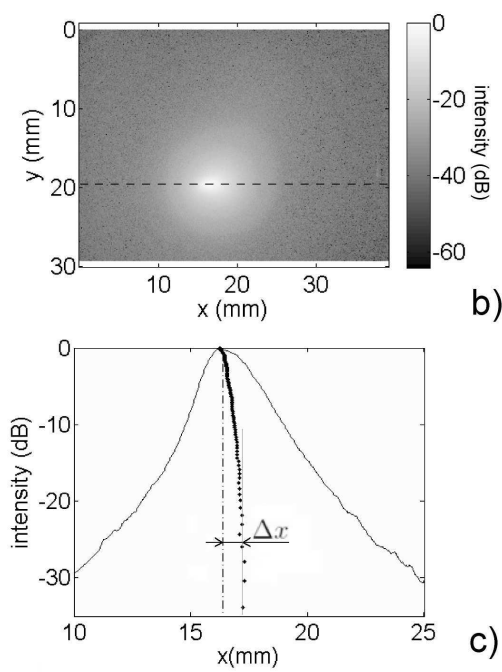

Figure 2.2: (a) Schematic of the oblique-incidence diffuse reflectance principle and setup. (b) Diffuse reflectance intensity map. (c) Diffuse reflectance profile (solid curve),calculated midpoint curve (dot) and light entry point (dash dot) at the position indicated in dash line in (b).

A large PVA phantom prepared with dimensions of $15 \times 10 \times 6 \mathrm{~cm}^{3}$, is divided into three blocks (Figure 2.1). Block I is used for measuring the $\mu_{s}^{\prime}$ (section 2.2.3). Block II is used for acoustic property measurements (section 2.2.4). Block III is used for the microstructure study with SEM (section 2.2.5). 


\subsubsection{Temperature measurements}

The temperature at two different locations (at the surface and $30 \mathrm{~mm}$ under the surface) inside the PVA gel during F-T cycles is simultaneously recorded using two K-type thermocouples (Rössel Messtechnik, Dresden, Germany).

\subsubsection{Reduced scattering coefficient assessment}

A custom-made setup based on the principle of oblique-incidence diffuse reflectance (ODR) is used to measure the reduced scattering coefficient $\left(\mu_{s}^{\prime}\right)$. The method is discussed in detail by Wang and Jaques [21], for a semi-infinite sample with $\mu_{s}^{\prime}$ much larger than the absorption coefficient $\mu_{a}$. Under these assumptions, a narrow beam incident obliquely on the surface can be approximated by a buried isotropic point source that is $1 / \mu_{s}^{\prime}$ away from the incident point (See Figure 2.2(a)). The center of the diffuse reflectance is shifted from the center of the incident beam by $\Delta x$. From the diffuse reflectance intensity map (Figure 2.2(b)), a profile is selected along the major axis of the elliptical spot (Figure 2.2(c)) from the edges of the reflectance profile at each intensity level. The spatial difference between the light entry point and the midpoint curve at saturation indicate the shift $\Delta x$. Considering also the refraction, $\Delta x$ is expressed as:

$$
\Delta x=\frac{\sin \left(\gamma_{\mathrm{t}}\right)}{\mu_{s}^{\prime}}
$$

where $\gamma_{t}$ is the refractive angle, calculated from the known incident angle $\gamma_{i}$ and refractive index of the sample $n$ by:

$$
\sin \left(\gamma_{\mathrm{i}}\right)=n \sin \left(\gamma_{\mathrm{t}}\right)
$$

In our experimental setup, as shown in Figure 2.2(a), a laser diode with excitation wavelength of $784 \mathrm{~nm}$ (Thorlabs, Newton, New Jersey, USA) is used as CW laser source. The laser beam is coupled through a $600 \mu \mathrm{m}$ fiber and collimated on the surface of the sample to obtain a beam diameter around $1 \mathrm{~mm}$. A CCD camera (Allied Vision technologies, Stadtroda, Germany) is used to image the beam shape. A neutral density filter is used to attenuate the imaged light intensity and to prevent saturation of pixels. The fiber coupling unit and the collimating lens together with the optical filter are fixed on a custom-made aluminum arm, which can be rotated to adjust the incident angle of the laser beam. For every measurement, two images, with and without filter are taken and an incident angle of $45^{\circ}$ is used.

A calibrated sample with known $\mu_{s}^{\prime}$ and refractive index $n$ is measured to validate our ODR system. This sample is based on epoxy resin with the optical scattering 


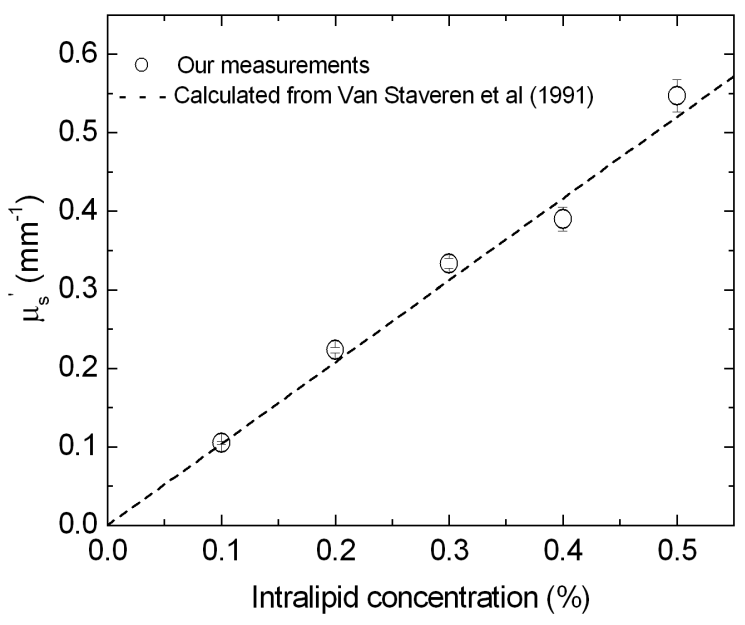

Figure 2.3: Reduced scattering coefficient $\left(\mu_{s}^{\prime}\right)$ at $784 \mathrm{~nm}$ for varying Intralipid $20 \%$ concentrations in water measured using the oblique-incidence diffuse reflectance setup. Our results are compared with $\mu_{s}^{\prime}$ values calculated from Van Staveren et al [22]. Each sample is measured 5 times, error bars represent standard deviations.

Table 2.1: Validation of the oblique-incidence diffuse reflectance system. The sample is calibrated at $800 \mathrm{~nm}$ and measured at $784 \mathrm{~nm}$. Five measurements are taken for the sample.

\begin{tabular}{lll}
\hline \hline & $\begin{array}{l}\text { Reduced scattering }\left(\mu_{s}^{\prime}\right) \\
\left(\mathrm{mm}^{-1}\right)\end{array}$ & $\begin{array}{l}\text { Reduced scattering }\left(\mu_{s}^{\prime}\right) \\
\left(\mathrm{mm}^{-1}\right)\end{array}$ \\
\hline Calibrated sample & $1.40 \pm 0.05^{\mathrm{a}}$ & $1.41 \pm 0.11^{\mathrm{b}}$ \\
\hline \hline
\end{tabular}

${ }^{\text {a }}$ From Firbank et al [46].

b Our measurements.

provided by adding a proper amount of $\mathrm{TiO}_{2}$ particles (Firbank and Delpy 1993). Good agreement between the measured $\mu_{s}^{\prime}$ and reported value is obtained (Table 2.1). Further, 20\% Intralipid (batch no. WI15893, Fresnius Kabi Nederland BV, The Netherlands) samples with concentration in water varying from $0.1-0.5 \%$ in volume were measured. As shown in Figure 2.3, our measurements show good agreement with the reported values calculated from Van Staveren et al [22].

The spatial distribution of $\mu_{s}^{\prime}$ within the PVA phantom is investigated by applying the ODR approach at different locations on three planes of block I as indicated in Figure 2.1. Plane $a$ is the surface of the block, plane $b$ is obtained by removing a $5 \mathrm{~mm}$ thick layer after measurements at plane $a$. Plane $c$ is the surface of the block opposite 
to plane $a$. Therefore, these three planes represent the depths of $0 \mathrm{~mm}, 5 \mathrm{~mm}$ and $50 \mathrm{~mm}$ along the $\mathrm{Y}$ axis. Each plane is investigated at 5 depths from $1-5 \mathrm{~cm}$ along the $\mathrm{Z}$ axis ( 5 times averaging). Care was taken to enable that the diffuse reflectance beam lay well within the sample without interference from edges. A refractive index of 1.36 for the PVA specimens is used according to Kharine et al [7].

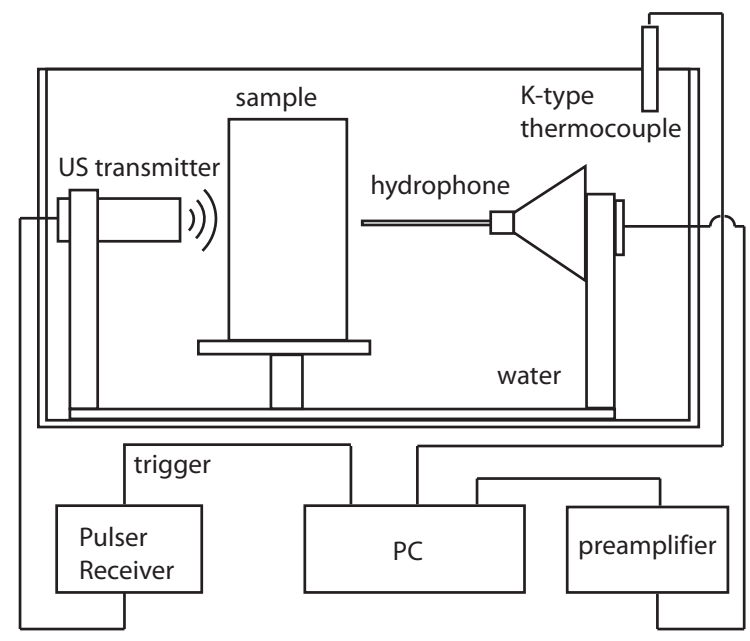

Figure 2.4: Schematic of the setup for speed of sound and acoustic attenuation measurements.

\subsubsection{Speed of sound and acoustic attenuation assessment}

The acoustic transmission properties are measured using the insertion technique [23]. A $5 \mathrm{MHz}$ unfocused single element transducer (V309 Panametrics) and a broadband needle hydrophone (BLLMCX074 Precision Acoustic Ltd. Dorchester) are mounted in a demineralized water bath at room temperature (Figure 2.4). The transducer is driven by a broadband ultrasonic pulser/receiver (Panametrics 5077PR) to emit an ultrasonic pulse whose frequency content is determined by the transducer center frequency and bandwidth $(2.25-7.8 \mathrm{MHz})$. The measurement data are recorded with a data acquisition card (U1067A Acqiris, 8 bit, $500 \mathrm{MS} / \mathrm{s}$ ) while the water temperature is logged using a USB thermocouple measurement device (NI-USB TC01) with the K-type thermocouple. The temperature remained stable within $\pm 0.05{ }^{\circ} \mathrm{C}$ during the measurement time of 5 minutes.

A reference signal is obtained when no sample is inserted between the transmitter 
and the receiver. The ultrasound pulse from the sample measurement has a timeof-arrival shift $\Delta T$ and amplitude decrease with respect to the reference signal. The thickness $\Delta d$ of each sample is measured several times around the position where the sample is insonified. For every sample the $\Delta d$ used in the estimation of the acoustic parameters is the mean \pm standard deviation of 10 measurements. Knowing the speed of sound in water $\left(C_{w}\right)$ at the measurement temperature [24], the frequency dependent SOS function $C_{s}(\omega)$ can be expressed in the frequency domain as:

$$
\frac{1}{C_{\mathrm{s}}(\omega)}=\frac{1}{C_{\mathrm{w}}}-\frac{\Delta \phi(\omega)}{\omega \Delta d}
$$

in which $\omega$ is the angular frequency, $\Delta \phi(\omega)$ is the phase difference between the FFT of the signals with and without sample insertion. Attenuation can be expressed as:

$$
\alpha_{\mathrm{s}}(\omega)=\alpha_{\mathrm{w}}-\frac{1}{\Delta d}\left[\ln A_{\mathrm{s}}(\omega)-\ln A_{\mathrm{w}}(\omega)\right]
$$

where $A_{\mathrm{i}}(\omega)$ is the amplitude in the frequency domain, $\alpha_{\mathrm{i}}(\omega)$ the attenuation coefficient $\left(\mathrm{dB} \mathrm{cm}^{-1} \mathrm{MHz}^{-1}\right)$ with $i=s, w$ (sample, water). The attenuation of most materials follows a frequency power law [25]:

$$
\alpha_{\mathrm{s}}(\omega)=\alpha_{0}\left|\omega^{\mathrm{y}}\right|
$$

where $y$ is the power law factor assumed to be 1 as in most soft tissues, and $\alpha_{0}$ is the attenuation constant. SOS and AA can be estimated simultaneously by using the Kramers-Krönig (KK) relation fitting the power law to the frequency bandwidth of the transducer [26-28]:

$$
\frac{1}{C_{\mathrm{s}}(\omega)}=\frac{1}{C_{\mathrm{s}}\left(\omega_{0}\right)}+\alpha_{0} \tan \left(\frac{\pi}{2} y\right)\left(|\omega|^{\mathrm{y}-1}-\left|\omega_{0}\right|^{\mathrm{y}-1}\right)
$$

From the data we sample both SOS and AA at the frequency of interest namely 5 $\mathrm{MHz}$. Errors due to interface losses will be minimized with this frequency domain approach since these are expected to be frequency independent.

The KK-based solver has been validated using two calibrated samples based on evaporated milk and agarose [29-31] provided by E. L. Madsen (University of Wisconsin, USA). The sample thicknesses were measured to be $22.95 \pm 0.24 \mathrm{~mm}$ and 20.85 $\pm 0.15 \mathrm{~mm}$ for sample I and II respectively. Good agreement is achieved between the measured SOS and AA with the calibrated values (Table 2.2).

Block II in Figure 2.1 is used for the measurements. It is divided into 5 pieces with the same size approximately of $2 \times 2 \times 1 \mathrm{~cm}^{3}$. Therefore, these five samples are representative of depths from $1 \mathrm{~cm}$ to $5 \mathrm{~cm}$. 


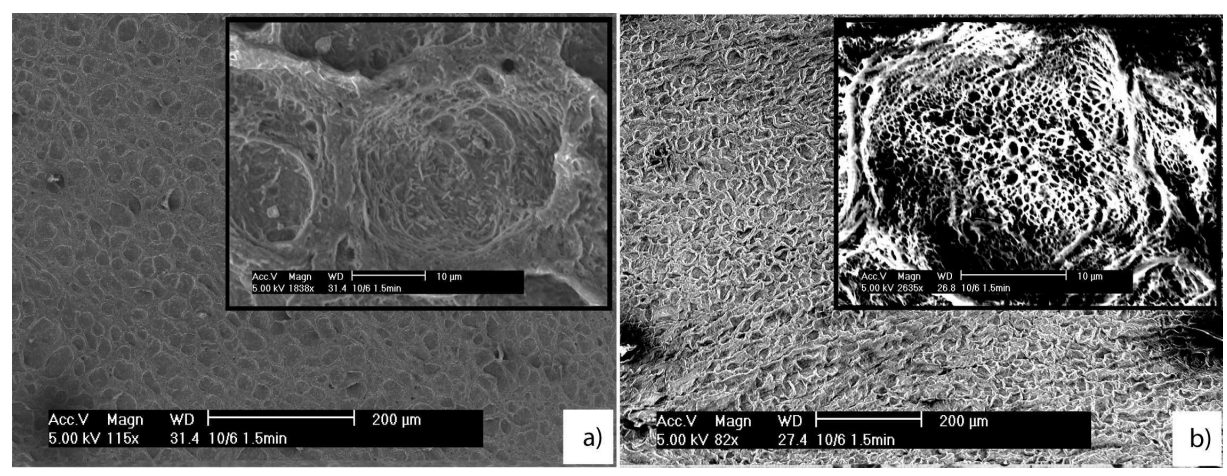

Figure 2.5: Scanning electron microscopy of (a) Outer surface of critical point dring processed sample and $(b)$ Liquid nitrogen processed sample.

\subsubsection{Microstructure of PVA gels}

Several authors [32-34] have studied PVA hydrogel microstructure. However, their specific applications did not require the cast sample to exceed $3 \mathrm{~mm}$ thickness. For breast mimicking phantoms, characterization needs to be done on samples with thickness in the order of a few centimeters.

Trials were carried out to choose the optimum procedure for pore analysis. In samples prepared by critical point drying (CPD) [32] and liquid nitrogen (LN2) treatment [33, 35], SEM (FEI/Philips XL30 ESEM ${ }^{\mathrm{TM}}$ FEG, FEI Company, Hillsboro, USA) inspection showed 10 to $20 \mu \mathrm{m}$ cavities on the surface of the specimens (Figure 2.5). However, under higher magnification of CPD treated samples (Figure 2.5(a) inset), the structures appeared collapsed (See also reference [33]). In LN2 treated samples (Figure 2.5(b) inset), smaller cavities are further distinguishable. LN2 treatment is thus preferred since the pores are preserved, but also since it is fast. Further the samples undergo spontaneous cracking into 2 or more fragments when quenched. This exposes the interiors avoiding the need for further cutting.

A $2 \mathrm{~cm}$ thick slice of the phantom, block III, is cut into four main blocks (Figure 2.1, group 1-4). Three specimens from each block are obtained (Figure 2.1). Every specimen is inspected with SEM at different locations; average pore size is determined from 150 pores. The number of pores in a $5 \times 5 \mu \mathrm{m}^{2}$ region of interest $\left(A_{R O I}\right)$ is counted for every specimen and is used to calculate the average wall thickness. From the number of pores $\nu$ and the average radius $\rho$, the total area occupied by assumed circular pores is:

$$
A_{\mathrm{P}}=\nu \pi \rho^{2}
$$


The area of material surrounding each pore, on average, can be expressed as:

$$
A_{\mathrm{W}}=\frac{A_{\mathrm{ROI}}-A_{\mathrm{P}}}{\nu}
$$

Finally, assuming the wall of each pore to be a circular ring, its thickness is given by:

$$
W=\sqrt{\frac{A_{\mathrm{W}}}{\pi}}
$$

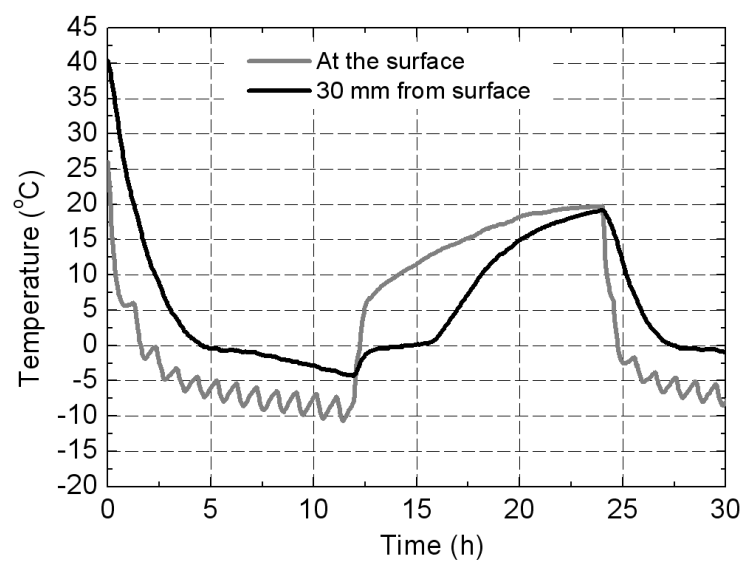

Figure 2.6: Temperature recorded at the surface (gray line) and $30 \mathrm{~mm}$ under the surface (black line) of a large PVA phantom during F-T cycles.

\section{$2.3 \quad$ Results}

\subsubsection{Temperature measurements}

Temperatures measured at the surface and the inside of the large PVA phantom during F-T procedure are shown in Figure 2.6. The aqueous PVA solution was allowed to stabilize for 2 hours before submitting it to the F-T procedure. At the instant when the exterior had cooled to room temperature, the interior was still warm. The thermocouple positioned close to the surface experiences the typical saw tooth temperature oscillations in the refrigerator produced by simple ON-OFF control exerted by the thermostat. The thawing cycle on the other hand shows a smooth temperature rise since this is not a thermostat controlled effect. The interior temperature lags the surface cooling, and at any instant in time is $5-10{ }^{\circ} \mathrm{C}$ higher than the surface temperature. When the surface temperature reaches the freezing temperature of water, 
the bulk temperature is still about $15^{\circ} \mathrm{C}$. The outer layer of the PVA phantom thus starts freezing earlier than the inside. The same phenomenon occurs during thawing. Both the surface temperature and bulk temperature measured do not reach $-20{ }^{\circ} \mathrm{C}$ when undergoing $12 \mathrm{~h}$ freezing.

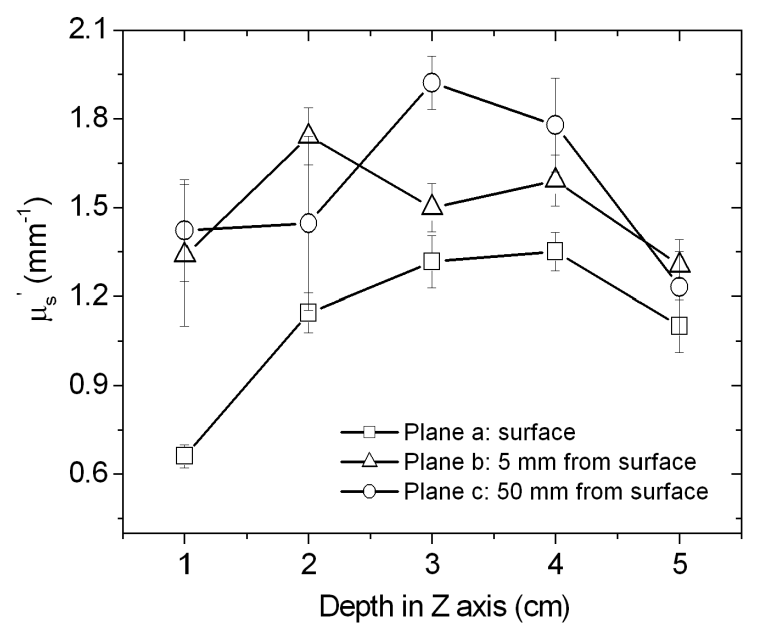

Figure 2.7: Measured $\mu_{s}^{\prime}$ distribution at $784 \mathrm{~nm}$ inside the large PVA phantom. Data points represent average $\mu_{s}^{\prime}$ value of 5 measurements from the plane a, plane $\mathrm{b}$ and plane $\mathrm{c}$ of the phantom shown in Figure 2.1. The error bars represent the standard deviations.

\subsubsection{Reduced scattering coefficient}

The $\mu_{s}^{\prime}$ distribution inside the large PVA phantom is shown in Figure 2.7. Three sets of measurements are performed for the three planes of block I (Figure 2.1). The measured $\mu_{s}^{\prime}$ on the PVA surface is generally lower than that measured in the bulk. Both plane $a$ and plane $c$ measurements show that $\mu_{s}^{\prime}$ increases approaching the center of the phantom and the maximum $\mu_{s}^{\prime}$ inside the phantom is approximately 3 times larger than that on the surface. The $\mu_{s}^{\prime}$ from plane $c$ at depths from $1-5 \mathrm{~cm}$ has a maximum variation of less than $30 \%$. However, the largest variation is within the first few millimeters from the surface layer. Measurements from plane $b$ and plane $c$ show similar behavior.

The $\mu_{s}^{\prime}$ values of the large PVA phantom at different locations measured using the ODR technique are dependent on the refractive index $n$ of the sample. According to Kharine et al [7], a refractive index of 1.36 for PVA is used for $\mu_{s}^{\prime}$ calculation. Since 
Table 2.2: Calibrated $\left(22^{\circ} \mathrm{C}\right)$ and measured $\left(22.7^{\circ} \mathrm{C}\right)$ speed of sound and acoustic attenuation values for the two calibrated samples. Temperatures were stable within $\pm 0.05{ }^{\circ} \mathrm{C}$ during the measurement time of 5 minutes. The mean and dispersions in measured thicknesses were used in the estimation.

\begin{tabular}{lllll}
\hline \hline & \multicolumn{2}{c}{ Calibrated } & \multicolumn{2}{c}{ Measured } \\
\hline Sample \# & $\begin{array}{l}\text { SOS } \\
\left(\mathrm{m} \mathrm{s}^{-1}\right)\end{array}$ & $\begin{array}{l}\text { AA@5MHz } \\
\left.(\mathrm{dB} \mathrm{cm})^{-1}\right)\end{array}$ & $\begin{array}{l}\text { SOS@5MHz } \\
\left(\mathrm{m} \mathrm{s}^{-1}\right)\end{array}$ & $\begin{array}{l}\text { AA@5MHz } \\
\left.(\mathrm{dB} \mathrm{cm})^{-1}\right)\end{array}$ \\
\hline I & $1559^{\mathrm{a}}$ & $5.87^{\mathrm{a}}$ & $1564 \pm 1.0$ & $5.4 \pm 0.2$ \\
$\mathrm{II}$ & $1535^{\mathrm{a}, \mathrm{b}}$ & $2.67^{\mathrm{a}, \mathrm{b}}$ & $1538 \pm 0.4$ & $2.4 \pm 0.1$ \\
\hline \hline
\end{tabular}

a Madsen (2008) private communication.

${ }^{\mathrm{b}}$ From Wear et al [31].

the temperature distribution might affect the refractive index, we recalculated the $\mu_{s}^{\prime}$ using a refractive index in the range between 1.33 (water) and 1.5 (most polymers). The corresponding variations are less than $10 \%$. This ensures a weak dependence of the determination of $\mu_{s}^{\prime}$ on refractive index variation.

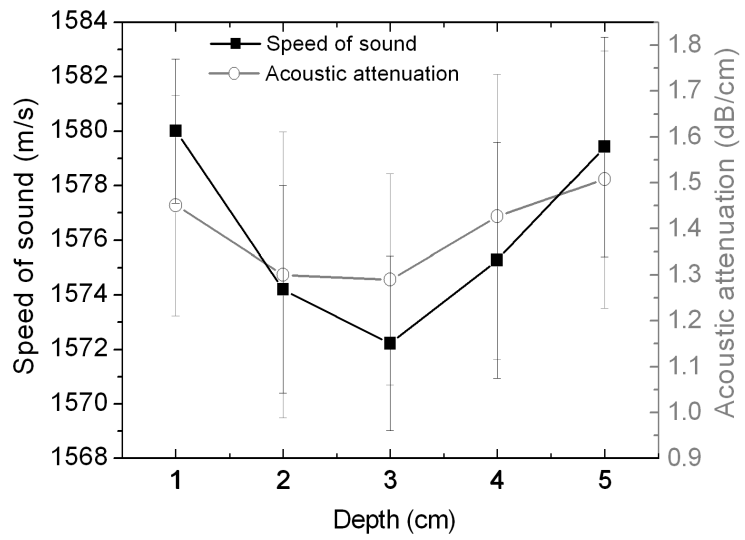

Figure 2.8: Speed of sound and acoustic attenuation (at $5 \mathrm{MHz}$ ) at different depths from the surface to the bulk of large PVA phantom at $22.5^{\circ} \mathrm{C}$. The data points represent the average values from 10 measurements, error bars represent the standard deviations. 


\subsubsection{Speed of sound and acoustic attenuation measurements}

The SOS and AA measured in specimens located at different depths from the surface down into the bulk of the large PVA phantom are shown in Figure 8. Though their standard deviations are relatively high due to roughness of the sample surfaces caused by cutting leading to dispersions in thickness estimation (standard deviations \pm 0.5 $\mathrm{mm}$ ), in general the SOS values obtained are comparable to the values reported for biological tissue. SOS is usually quoted as between 1425-1575 $\mathrm{m} \mathrm{s}^{-1}$, [23] and AA between $0.5-1.1 \mathrm{~dB} \mathrm{~cm}^{-1}$ at $1.76 \mathrm{MHz}$ [36]. Both SOS and AA decrease slightly from the surface towards the center of the phantom. The spatial variation in the acoustic properties however is within $1 \%$ for SOS and less than around $13 \%$ for AA.

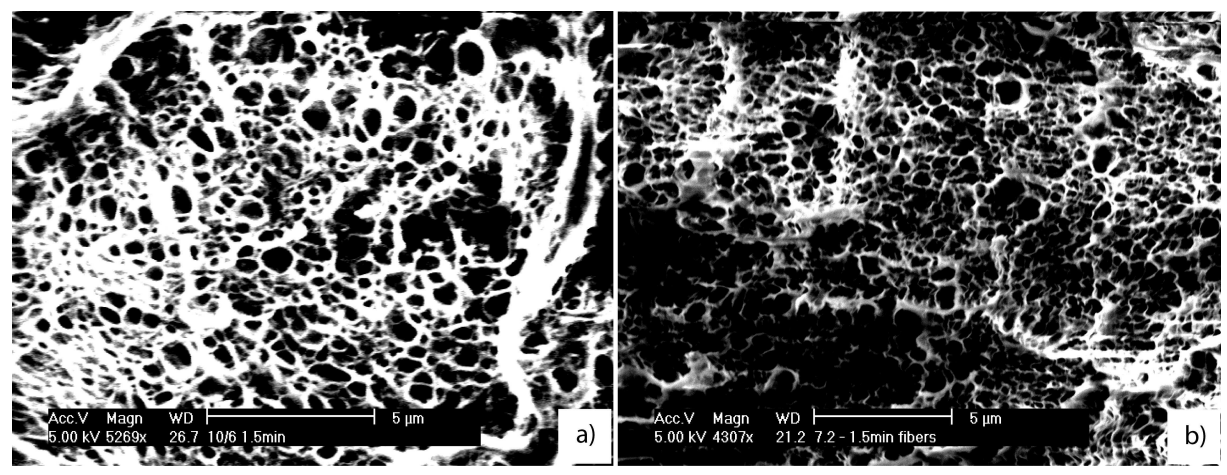

Figure 2.9: Scanning electron microscope imaging of liquid nitrogen preprocessed specimens situated at (a) surface and (b) a deep lying cross-sections.

\subsubsection{Microstructures of PVA gels}

Besides 10 to $20 \mu \mathrm{m}$ large cavities on the surface (Figure 2.5), the SEM micrograph of a deep cross-section and of the surface show similar small scale pores (less than 1 $\mu \mathrm{m})$ almost uniformly distributed throughout the entire specimen area (Figure 2.9). Figure 2.10(b) summarizes the average pore size at the locations specified in Figure 2.10(a) with their standard deviation. It also compares these dimensions with the small scale pores which are located at the outer surface (sample 0 in Figure 2.10(a) and (b)). Within the same specimen, the pore size variability is considerable, but the overall distribution does not exhibit any particular trend. The average pore size in the bulk is sub-micrometer and smaller than in Hyon et al [37] if the large 10-20 $\mu \mathrm{m}$ structures present at the outer surfaces are discarded. 
Estimates of the wall thickness are shown in Figure 2.10(c) along with the number of pores in the specified ROI. The pore size does not change, but a difference is found in density and wall thickness. Bulk specimens (group 3 and 4) exhibit lower wall thickness (approximately -25\%) than side specimens (group 1 and 2) as shown in Figure 2.10(d). Thus, pore density increases towards the center of the phantom.

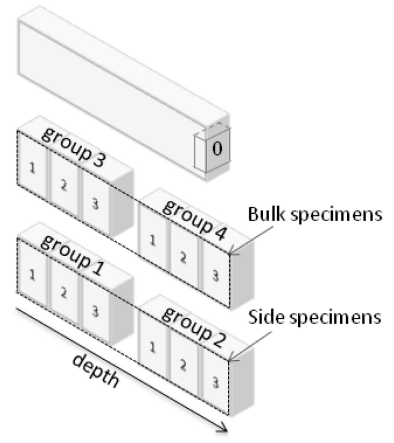

(a)

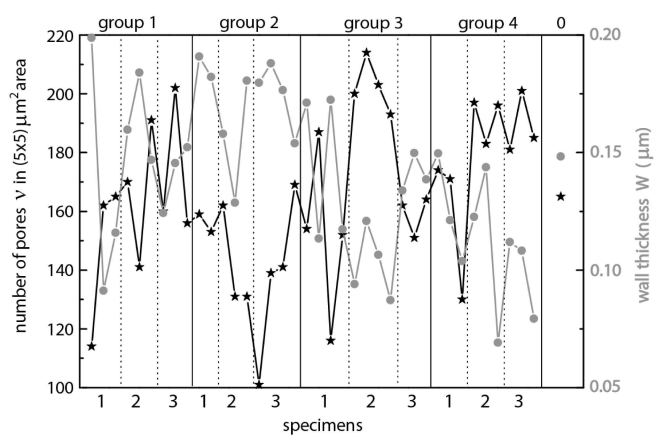

(c)

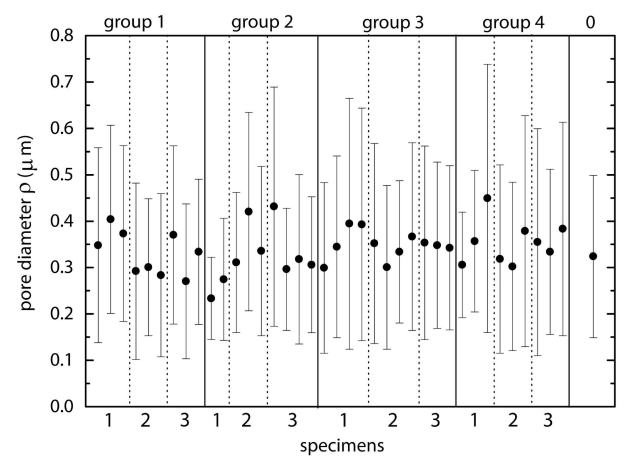

(b)

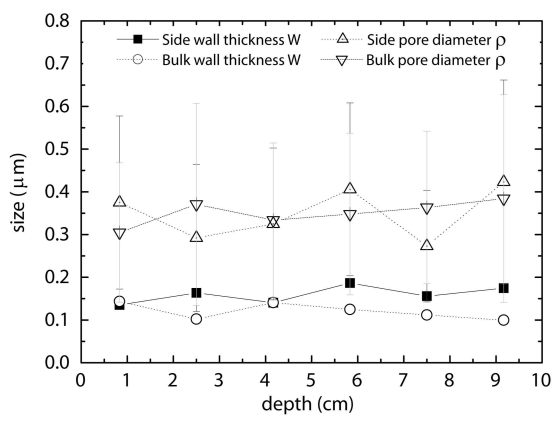

(d)

Figure 2.10: (a) Schematic of block III in Figure 2.1, (b) Pore sizes in the locations specified in (a), the error bars represent the standard deviations. (c) Pore density (black stars, left axis) and estimate of wall thickness (gray dots, right axis) for all specimens. (d) Pore diameter and wall thickness for grouped sample in depth. The error bars represent the standard deviations.

\subsection{Discussion}

Kharine et al [7] introduced PVA as a suitable material for photoacoustic mammography utilizing its known acoustic property similarity with tissue, while fine tuning 
its optical properties. The optical properties were characterized in that work on specially prepared small test specimens. Since the gels are formed using F-T it is to be expected that temperature responses of larger gels will be spatially non-uniform. We also investigated spatial distribution of optical (and acoustic) properties as this may be related to the temperature during the F-T process. As the optical absorption is only dependent on the molecular structure of the material, the intrinsic absorption is not F-T dependent, and at the wavelength of interest it is appropriate for breast tissue phantom [7]; this parameter was not measured during the experiments. We focused on optical reduced scattering coefficient, speed of sound and acoustic attenuation. The $\mu_{s}^{\prime}$, was measured using ODR. Finally, we inspected the microstructure using scanning electron microscopy.

Temperature monitoring showed significant differences from the surface to the bulk (Figure 2.6). When the temperature monitoring is begun, with the aqueous PVA solution in the mould, there is a difference of $15^{\circ} \mathrm{C}$ between the two thermocouple readings. When the surface temperature reaches water freezing point, the bulk temperature is still above $10^{\circ} \mathrm{C}$. It takes a further $3 \mathrm{~h}$ for the bulk to start freezing.

In general, the values of $\mu_{s}^{\prime}$ measured are in the range of values quoted for breast tissue [38]. There is a substantial difference between surface and interior values (0.6$1.9 \mathrm{~mm}^{-1}$ ) (See Figure 2.7). Within the bulk the maximum variation is about $30 \%$ $\left(1.2-1.9 \mathrm{~mm}^{-1}\right)$. From the distribution, a two-layer structure may be observed: a center region with higher values and an outer shield region of a roughly $5 \mathrm{~mm}$ thickness having lower values. It is interesting that this structure matches the standard twolayer breast model used for dosimetry in x-ray mammography [39]. The breast is modelled as having an inner region composed of a mixture of adipose and glandular tissue, and an outer layer of predominantly adipose tissue of $5 \mathrm{~mm}$ thickness. We surveyed the literature concentrating on $\mu_{s}^{\prime}$ distribution for breast in vivo at the wavelength range we used. Brooksby et al [40] imaged the distribution in breast in vivo using MRI guided near-infrared tomography at wavelength of $785 \mathrm{~nm}$. MRI image shows the breast to be clearly divided into an outer adipose tissue layer and an internal glandular area. Comparing MRI findings with the scattering distribution it can be observed that a low scattering area $\left(0.93 \mathrm{~mm}^{-1}\right)$ is associated to the adipose tissue, and a higher scattering area $\left(1.12 \mathrm{~mm}^{-1}\right)$ matches the glandular tissue (see Figure 2.11). Similar behavior has been reported in references $[41,42]$ in vivo and references $[38,43,44]$ ex vivo (see Table 2.3 ). Thus we find that the PVA gel sample fortuitously mimics the distribution of adipose tissue and glandular tissue in the breast. While this makes it then an excellent breast phantom, in applications where 

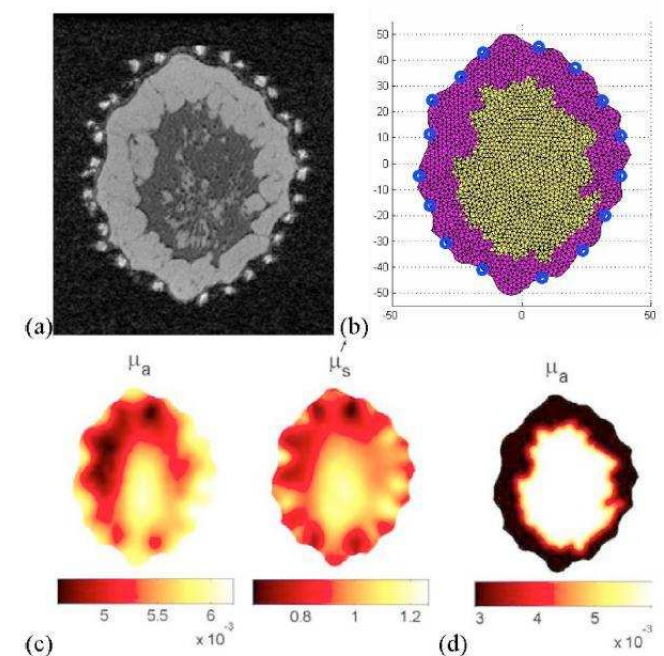

(c)
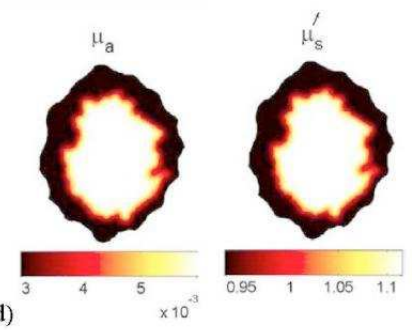

Figure 2.11: In (a), an anatomically coronal T1-weighted MRI displays adipose (outer) and glandular (inner) tissue types. A two layer structured FEM mesh and source locations, created from (a), is shown in (b). Absorption and reduced scattering coefficient reconstructions in (c) are obtained without utilizing the internal structure of (b). In (d), MRI data guides a two-region parameter fitting algorithm. Relative to (c), resolution has improved and contrast has increased, showing higher absorption and scatter in glandular relative to adipose tissue. Reproduced from Ref. [40] with permission.

more homogeneous samples are required the $5 \mathrm{~mm}$ thick surface layer can be removed using a scalpel.

The SOS and AA values show relatively homogeneous distribution (Figure 2.8) with slight decrease in values towards the center. The values and their variations are within the range of acoustic properties of the breast.

Microstructural inspection of the surface has revealed two different scale structures: large cavities of several $\mu \mathrm{m}$ and these filled with sub- $\mu \mathrm{m}$ pores. In the interior we found only the smaller pores with an average diameter of $0.35 \mu \mathrm{m}$. Even though the pore-size dispersion is quite high, no trend is observed as a function of location whether at the surface or in the interior. However, we observe a difference in the spacing between pores in the bulk, with pores being closer-packed towards the center. (See Figure 2.10(c) and (d)).

We expect that because pore densities increase, while largely maintaining the same diameters, the interiors will possess a higher water volume fraction. This can explain the lower SOS and AA measured in the bulk (see also Ref. [45]). The pore 
Table 2.3: Our results compared with literature values for the optical reduced scattering $\mu_{s}^{\prime}$ in adipose and glandular tissue.

\begin{tabular}{lll}
\hline \hline $\begin{array}{l}\text { adipose tissue } \\
\mu_{s}^{\prime}\left(\mathrm{mm}^{-1}\right)\end{array}$ & $\begin{array}{l}\text { glandular tissue } \\
\mu_{s}^{\prime}\left(\mathrm{mm}^{-1}\right)\end{array}$ & status \\
\hline $0.6-1.2$ & $1.2-1.9$ & phantom - our measurements at $784 \mathrm{~nm}$ \\
$0.7 \pm 0.1$ & $1.1 \pm 0.2$ & ex vivo $400-600 \mathrm{~nm}$ derived from Ref. [43] \\
0.93 & 1.2 & in vivo at $785[40]$ \\
$0.86 \pm 0.13$ & $1.43 \pm 0.3$ & ex vivo at $700 \mathrm{~nm}[38]$ \\
0.645 & 1.128 & ex vivo at $600 \mathrm{~nm}[44]$ \\
\hline \hline
\end{tabular}

density variations are also responsible for the observed spatial distribution of optical properties: the pore-denser regions provide more scattering events which explains the slight $\mu_{s}^{\prime}$ increase towards the center while the pore sizes are relatively constant in the bulk. At the surface layers the converse occurs with relatively lower pore densities and further, the larger 10-20 $\mu \mathrm{m}$ cavities show lower $\mu_{s}^{\prime}$ than the wavelength-sized sub-micron pores inside.

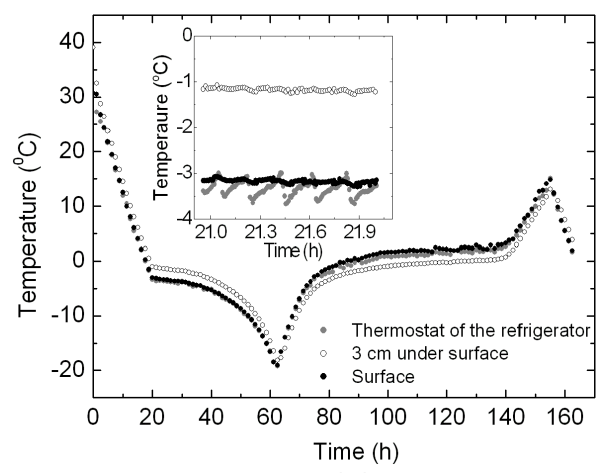

(a)

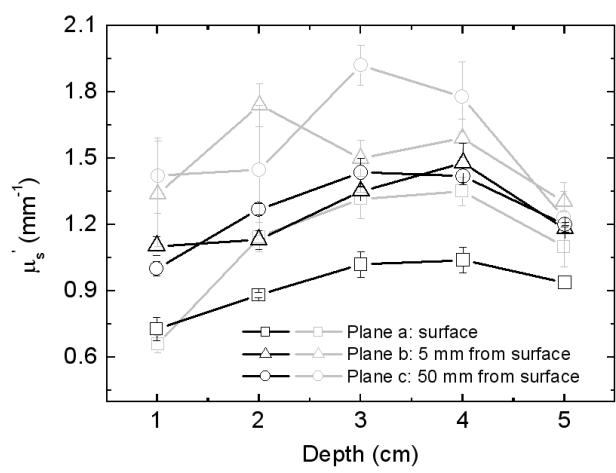

(b)

Figure 2.12: (a) Temperature of a large sample prepared using controlled FT recorded at surface, $3 \mathrm{~cm}$ under the surface and the set temperature for surface. (b) Optical reduced scattering $\mu_{s}^{\prime}$ distribution measured using the oblique-incidence diffuse reflectance setup for a large PVA sample prepared without (gray) and with (black) temperature controlled FT.

The reason for the spatial distribution of pore characteristics is not yet understood. We made a first effort to resolve this by exposing the entire volume of aqueous PVA solution and the forming gel to a uniform temperature. The F-T cycles were applied 
using an external temperature control of the refrigerator, where the rate of cooling (warming) was set to $2^{\circ} \mathrm{C} /$ hour while the difference between surface and interior thermocouple readings was set to be less than $2^{\circ} \mathrm{C}$. A freezing cycle in such a case took 60 hour till the block was uniformly cooled to $-20^{\circ} \mathrm{C}$ (Figure $2.12(\mathrm{a})$ ). The distribution of optical reduced scattering was only marginally better (see Figure 2.12(b)). This suggests that the mechanism responsible for the variation in pore characteristics may have to do with internal stresses exerted at the interior compared to the outer layer rather than only due temperature or temperature gradients experienced.

\subsection{Conclusion}

We observed substantial temperature differences between the shallow regions and the interiors during the freezing and thawing procedure to fabricate a large poly(vinyl alcohol) gel phantom. We studied the optical and acoustic properties sampled at various locations of such a phantom prepared by 4 freezing-thawing cycles. In general the acoustic and optical properties of the phantom matched values quoted for breast tissue. The spatial distribution of the acoustic properties namely the speed of sound and acoustic attenuation were relatively uniform. On the other hand, the reduced optical scattering coefficient showed significant differences between the surface and the bulk. This distribution fortuitously matches the optical properties of the two-layer model chosen for representing the breast with an inner region composed of an adipose tissue-glandular tissue mixture, and an outer layer of predominantly adipose tissue. If this optical property variation is not acceptable in an application, we recommend removal of the superficial layer to a depth of around $5 \mathrm{~mm}$. 


\section{Bibliography}

[1] R. J. Nordstrom, "Phantoms as standards in optical measurements," Proceeding of SPIE 7906B, 26 (2011).

[2] L. V. Wang, "Multiscale photoacoustic microscopy and computed tomography," Nature photonics 3, 503-509 (2009).

[3] D. Piras, W. Xia, W. Steenbergen, T. G. van Leeuwen, and S. Manohar, "Photoacoustic imaging of the breast using the twente photoacoustic mammoscope: Present status and future perspectives," IEEE J. Selec. Topic Quant. Electron 16, 730-739 (2010).

[4] R. A. Kruger, R. B. Lam, D. R. Reinecke, S. P. D. Rio, and R. P. Doyle, "Photoacoustic angiography of the breast," Med. Phys. 37, 6096-6100 (2010).

[5] S. A. Ermilov, T. Khamapirad, A. Conjusteau, M. H. Leonard, R. Lacewell, K. Mehta, T. Miller, and A. A. Oraevsky, "Laser optoacoustic imaging system for detection of breast cancer," J. Biomed. Opt. 14(2), 024007 (2009).

[6] T. D. Kholhlova, I. M. Prelivanov, V. V. Kozhushko, A. N. Zharinov, V. S. Solomatin, and A. A. Karabutov, "Optoacousitc imaging of absorbing objects in a turbid medium: ultimate sensitivity and application to breast cancer diagnostics," Appl. Opt. 46, 262-272 (2007).

[7] A. Kharine, S. Manohar, W. Steenbergen, R. G. M. Kolkman, R. A. Bolt, and F. F. M. de Mul, "Poly(vinyl alcohol) gels for use as tissue phantoms in photoacoustic mammography," Phys. Med. Biol 48, 357-370 (2003).

[8] S. Manohar, A. Kharine, J. C. G. van Hespen, W. Steenbergen, F. F. M. de Mul and T. G. van Leeuwen, "Photoacoustic imaging of inhomogeneities embedded in breast tissue phantoms," Proceedings of SPIE-The international society for Optical Engineering 4960, 64-75 (2003). 
[9] S. Sethuraman, S. Aglyamov, J. Amirian, R. Smalling, and S. Emelianov, "Intravascular photoacoustic imaging to detect and differentiate atherosclerotic plaques," Proceeding of the IEEE international Ultrasonics Symposium , 133136 (2005).

[10] J. L. Su, B. Wang, and S. Y. Emelianov, "Photoacoustic imaging of coronary artery stents," Optics Express 17, 19894-19901 (2009).

[11] C. U. Devi, R. M. Vasu, and A. K. Sood, "Design, fabrication, and characterization of a tissue-equivalent phantom for optical elastography," J. Biomed. Opt. 10(4), 044020 (2005).

[12] C. M. Hassan, and N. A. Peppas, "Structure and application of poly(vinyl alcohol) hydrogels produced by conventional crosslinking or by freezing/thawing methods," Adv. Polymer. Sci. 153, 37-65 (2000).

[13] V. Pazos, R. Mongrain, and J. C. Tardif, "Polyvinyl alcohol cryogel: Optimizing the parameters of cryogenic treatment using hyperelastic models," J. Mech. Behav. Biomed. Mater. 2, 542-549 (2009).

[14] M. Nambu, T. Watari, T. Sakamoto, and K. Akojima, "Method for applying electromagnetic wave and ultrasonic wave therapies," US Patent, 4,958,626 (1990).

[15] K. Zell, J. I. Sperl, M. W. Vogel, R. Niessner, and C. Haisch, "Acoustic properties of selected tissue phantom materials for ultrasound imaging," Phys. Med. Biol. 52, 475-484 (2007).

[16] I. Mano, H. Goshima, M. Nambu, and M. Lio, "New polyvinyl alcohol gel material for mri phantoms," Magn. Reson. Med. 3, 921-926 (1986).

[17] K. C. Chu, and B. K. Rutt, "Polyvinyl alcohol cryogel: an idea phantom material for mr studies of arterial flow and elasticity," Magn. Reson. Med. 37, 314-9 (1997).

[18] S. Manohar, A. Kharine, J. C. G. van Hespen, W. Steenbergen, and T. G. van Leeuwen, "Photoacoustic mammography laboratory prototype: imaging of breast tissue phantoms," J. Biomed. Opt. 9, 1172 (2004).

[19] V. I. Lozinsky and L. Damshkaln, "Study of cryostructuration of polymer systems. xvii. poly(vinyl alcohol) cryogels: Dynamics of the cryotropic gel formation," J. Appl. Phys. 77, 2017-2023 (2000). 
[20] N. A. Peppas, "Turbidimetric studies of aqueous poly(vinyl alcohol) solutions," Makromol. Chem. 176, 3433-3340 (1975).

[21] L. Wang and S. L. Jaques, "Use of a laser beam with an oblique angle of incidence to measure the reduced scattering coefficient of a turbid medium," Appl. Opt. 13, 2362-2366 (1995).

[22] H. J. van Staveren, C. J. M. Moes, J. van Marie, S. A. Prahl, and M. J. C. van Gemert, "Light scattering in intralipid-10\% in the wavelength range of 400 Ü1100 nm," Appl. Opt. 30, 4507-4514 (1991).

[23] J. Bamber, Acoustic characteristics of biological media, New York: Wiley (1997).

[24] J. Lubbers and R. Graaff, "A simple and accurate formula for the sound velocity in water," Ultrasound Med. Biol. 24, 1065-1069 (1998).

[25] K. R. Waters and J. G. Miller, "Causality-imposed (kramers-krönig) relationships between attenuation and dispersion," IEEE Trans. Ultrason. Ferro Freq. Control 52, 822-834 (2005).

[26] K. R. Waters, M. S. Hughes, J. Mobley, G. H. Brandenburger, and J. G. Miller, "On the applicability of kramers-kronig relations for ultrasonic attenuation obeying a frequency power law," JASA 108, 556-568 (2000).

[27] K. V. Gurumurthy and R. M. Arthur, "A dispersive model for the propagation of ultrasound in soft tissue," Ultrasonic Imaging 4, 355-377 (1982).

[28] R. G. Willemink, S. Manohar, K. H. Slump, F. van der Heijden, and T. G. van Leeuwen, "A maximum likelihood method for obtaining integrated attenuation transmission mode measurements," Acoustics'08 Paris (2008).

[29] E. L. Madsen, G. R. Frank, and F. Dong, "Liquid or solid ultrasonically tissuemimicking materials with very low scatter," Ultrasound in Medicine and Biology 24, 535-542 (1998).

[30] R. B. Chin, E. L. Madsen, J. A. Zagzebski, H. Jadvar, X. K. Wu, and G. R. Frank, "A reusable perfusion supporting tissue-mimicking material for ultrasound hyperthermia phantoms," Med Phys 17, 380-390 (1990).

[31] K. A. Wear, T. A. Stiles, G. R. Frank, E. L. Madsen, F. Cheng, E. J. Feleppa, C. S. Hall, B. S. Kim, P. Lee, W. D. O'Brien, O. Jr, L. Michael, B. I. Raju, K. K. 
Shung, T. A. Wilson, and J. R. Yuan, "Interlaboratory comparison of ultrasonic backscatter coefficient measurements from 2 to $9 \mathrm{MHz}$," J Ultrasound Med $\mathbf{2 4}$, 1235-2366 (2005).

[32] S. H. Hyon and Y. Ikada, "Porous and transparent poly(vinyl alcohol) gel and method of manufacturing the same," US Patent, 4,663,358 (1987).

[33] H. H. Trieu and S. Qutubuddin, "Polyvinyl alcohol hydrogels I microscopic structure by freeze-etching and critical point drying techniques," Colloid Polym. Sci. 10, 3015-3022 (1994).

[34] L. Martin, M. Alonso, A. Girotti, F. J. Arias, and C. Rodriguez-Cabello, "Synthesis and characterization of macroporous thermosensitive hydrogels from recombinant elastin-like polymers," Biomacromolecules 272, 301-309 (2009).

[35] F. Yokoyama, and L. M. Shimamura, T. Ikawa, and K. Monobe, "Morphology and structure of highly elastic poly(vinyl alcohol) hydrogel prepared by repeated freezing-and-melting," Colloid and Polymer Science 264, 564-601 (1986).

[36] F. A. Duck, Physical Properties of Tissue: A Comprehensive Reference Book, London: Academic (1990).

[37] S. H. Hyon, W. Cha, and Y. Ikada, "Preparation of transparent poly(vinyl alcohol) hydrogel," Polymer Bull. 29, 119-122 (1989).

[38] V. G. Peters, D. R. Wyman, M. S. Patterson, and G. L. Frank, "Optical properties of normal and diseased human breast tissues in the visible and near infrared," Phys. Med. Biol. 35, 1317-1334 (1990).

[39] D. R. Dance, "Monte carlo calculation of conversion factors for the estimation of mean glandular breast dose," Phys. Med. Biol. 35, 1211-1219 (1990).

[40] B. Brooksby, S. J. Jiang, H. Dehghani, B. W. Pogue, K. D. Paulsen, C. Kogel, M. Doyley, J. B. Waeaver, and S. P. Poplack, "Magnetic resonance-guided nearinfrared tomography of the breast," Rev. Sci. Instrum. 75, 5262-5270 (2004).

[41] Q. Fang, S. A. Carp, J. Selb, G. Boverman, Q. Zhang, D. B. Kopans, R. H. Moore, E. L. Miller, D. H. Brooks, and D. A. Boas, "Combined optical imaging and mammography of the healthy breast: Optical contrast derived from breast structure and compression," IEEE transaction on medical imaging 28, 30-42 (2009). 
[42] Q. Fang, J. Selb, S. A. Carp, G. Boverman, E. L. Miller, D. H. Brooks, R. H. Moore, D. B. Kopans, and D. A. Boas, "Combined optical and x-ray tomosynthesis breast imaging," Radiology 258, 89-97 (2010).

[43] G. M. Palmer, C. Zhu, T. M. Breslin, F. Xu, K. W. Gilchrist, and N. Ramanujam, "Monte carlo-based inverse model for calculating tissue optical properties. part ii: Application to breast cancer diagnosis," Appl. Opt. 45, 1072-1078 (2006).

[44] H. L. Fu, B. Yu, J. Y. Lo, G. M. Palmer, T. F. Kuech, and N. Ramanujam, "A low-cost, portable, and quantitative spectral imaging system for application to biological tissues," Optics Express 18, 12630-12644 (2010).

[45] P. Chiarelli, A. Lanatä, and M. Carbon, "Acoustic waves in hydrogels: A biphasic model for ultrasound tissue-mimicking phantom," Materials Science and Engineering C 29, 899-907 (2009).

[46] M. Firbank and D. T. Delpy, "Design for a stable and reproducible phantom for use in near infra-red imaging and spectroscopy," Phys. Med. Biol. 38, 847-853 (1993). 



\section{Chapter 3}

\section{An optimized ultrasound detector for photoacoustic breast tomography}

3 Abstract

Photoacoustic imaging has proven to be able to detect vascularization-driven optical absorption contrast associated with tumors. In order to detect breast tumors located a few centimeter deep in tissue, a sensitive ultrasound detector is of crucial importance for photoacoustic mammography. Further, because the expected photoacoustic frequency bandwidth (a few $\mathrm{MHz}$ to tens of $\mathrm{kHz}$ ) is inversely proportional to the dimensions of light absorbing structures $(0.5$ to $10+\mathrm{mm})$, proper choices of materials and their geometries and proper considerations in design have to be made to implement optimal photoacoustic detectors. In this study, we design and evaluate a specialized ultrasound detector for photoacoustic mammography. Based on the required detector sensitivity and its frequency response, a selection of active material and matching layers and their geometries is made leading to a functional detector models. By iteration between simulation of detector performances, fabrication and experimental characterization of functional models an optimized implementation is made and evaluated. For computer simulation, we use 1D Krimholtz-LeedomMatthaei (KLM) and 3D finite-element (FEM) based models. The experimental

\footnotetext{
${ }^{3}$ This chapter has been published as: W. Xia, D. Piras, J. Van Hespen, S. Van Veldhoven, C. Prins, T. Van Leeuwen, W. Steenbergen and S. Manohar, "An optimized ultrasound detector for photoacoustic breast tomography," Med. Phys. 40(3), (2013), 032901. Reproduced with permission.
} 
results of the designed first and second functional detectors matched with the simulations. In subsequent bare piezoelectric samples the effect of lateral resonances was addressed and their influence minimized by sub-dicing the samples. Consequently, using simulations, a final optimized detector was designed, with a center frequency of $1 \mathrm{MHz}$ and a $-6 \mathrm{~dB}$ bandwidth of $0.4-1.25 \mathrm{MHz}$ (fractional bandwidth of $\sim 80 \%$ ). The detector should be capable of detecting vascularized tumors with 1-2 mm resolution. The measured minimum detectable pressure is $0.5 \mathrm{~Pa}$, which will facilitate deeper imaging compared to the current systems. Further improvements by proper electrical grounding and shielding and implementation of this design into an arrayed detector will pave the way for clinical applications of photoacoustic mammography.

\subsection{Introduction}

Photoacoustic imaging is an intrinsically hybrid biomedical imaging modality which is based on light excitation and ultrasound detection. In photoacoustics, short-pulsed laser light diffusively penetrates into tissue, is selectively absorbed by specific chromophores, such as hemoglobin, causing ultrasound waves to be generated by thermoelastic expansion. These are then detected by ultrasound detectors at the surface of tissue. Since ultrasound scattering is considerably lower than light scattering in biological tissue, the information carried by ultrasound waves arrives with less losses and distortion at the tissue surface than light waves would do. Thus photoacoustic imaging takes the advantages of both optical imaging and ultrasound imaging, with a high optical contrast, a high ultrasound resolution and large imaging depths [1-6]. One of the most important applications of photoacoustics is breast imaging, where the optical contrast comes from higher average hemoglobin levels associated with malignant masses compared to healthy breast tissue $[7,8]$. The technique promises to be an alternative to ionizing $\mathrm{x}$-ray and low contrast ultrasound imaging to detect breast cancer [9-14].

The faithful and sensitive detection of ultrasound (US) lies at the heart of a photoacoustic imaging system. The US detector largely determines image contrast, resolution and imaging depth of the system. In photoacoustic (PA) imaging of the breast, there is a requirement to detect tumors located a few centimeter deep in tissue, where light is heavily attenuated. Thus a sensitive ultrasound detector is of crucial importance. Further, the frequency range of photoacoustic waves is inversely proportional to the dimensions of absorbing structures [15]. In breast tissue, structures of interest range from 0.5 to $10+\mathrm{mm}$ initiating ultrasound frequencies from a few $\mathrm{MHz}$ 
down to tens of $\mathrm{kHz}[16]$. Thus a broadband US detector is required, centered on an optimum frequency.

Various US detectors with different specifications have been employed in photoacoustic (thermoacoustic) systems for breast imaging [10-12, 15, 17-20]. In 2000, Kruger et al. [17], reported the first thermoacoustic (TA) breast scanner in CT configuration. The US detector used 64 commercially available immersion detectors (1-3 piezocomposite, model 3847, Panametrics, Waltham, Mass.) each with flat face and a diameter of $13 \mathrm{~mm}$, arrayed in a spiral pattern spanning the surface of a hemispherical bowl. The detector had a center frequency of $1 \mathrm{MHz}$ with a fractional frequency bandwidth (FBW) of $70 \%$. The sensitivity of the detector was not reported. A later version of the system from 2010 [10], employed an array of 128 detectors (1-3 piezocomposite), $3 \mathrm{~mm}$ in diameter with a $5 \mathrm{MHz}$ center frequency and a FBW of $70 \%$. Submillimeter breast vasculature down to a depth of $40 \mathrm{~mm}$ was successfully visualized [10]. The sensitivity was not reported. However, the authors recommended the use of detectors with a lower center frequency to have greater imaging sensitivity.

Pramanik et al. [12] in 2008, reported a breast cancer detection system combining TA and PA tomography. They used 13-mm/6-mm-diameter active area non-focused detectors operating at $2.25 \mathrm{MHz}$ center frequency (piezocomposite, ISS $2.25 \mathrm{x} 0.5$ COM, Krautkramer) for signal detection. The detectors used have a large active surface area and a relatively low center frequency to gain a high sensitivity. The FBW is reported as varing between $60-120 \%$ but no details of sensitivity are reported.

The laser optoacoustic imaging system (LOIS) was developed over a decade ago and has undergone several iterations $[11,18,19]$. The latest system uses an array of 64 wideband polyvinylidene fluoride (PVDF) (bandwidth upto $2.5 \mathrm{MHz}$ ) elements arranged in a concave arc. Each detector element has a rectangular surface, with a large aspect ratio $(20 \mathrm{~mm} \times 3 \mathrm{~mm})$. This design gives the detector a slice-shape focusing area, providing high sensitivity in the region of interest. The sensitivity of this system was reported to be $1.66 \mathrm{mV} / \mathrm{Pa}$ at $1.5 \mathrm{MHz}$, the minimum detectable pressure (MDP) was not reported [11]. However, for some earlier versions the MDP values have been presented including measured [21] and estimated using only the thermal noise generated by the detector capacitance according to the Nyquist law [19, $22]$.

The Twente Photoacoustic Mammoscope (PAM) has been previously developed in our group in 2004 [24]. US detection uses a planar array of 590 PVDF detector elements. Each element has a $2 \mathrm{~mm}$ x $2 \mathrm{~mm}$ active surface area. The detector has a center frequency of $1 \mathrm{MHz}$ and a fractional bandwidth of $130 \%$ [20]. The measured 
MPD value is $80 \mathrm{~Pa}$ [9]. Promising clinical measurement results have been reported in 2007 and 2012 [14,25]. However, the system still suffers from the relatively low sensitivity of the PVDF detector.

PVDF detector arrays give LOIS and PAM broad bandwidths, while the measured sensitivity of the detectors are generally low compared to PZT detectors due to the reduced electromechanical coupling coefficients [23]. However, PZT detector suffers from a relatively low bandwidth. As a compromise, piezocomposite detectors provide better sensitivity and reasonably good FBW as employed by Kruger et al. [10] and Pramanik et al. [12].

To achieve a high sensitivity of the detector, we choose a highly sensitive PZT material, while tailoring the bandwidth of the detector to be reasonably wide. A single element PZT detector structurally consists of the active piezoelectric material, frontand back-matching layers and a backing layer. To have both high sensitivity and broad bandwidth, the materials, their acoustic characteristics and their dimensions should be carefully chosen. Furthermore, the aperture of the detector should be optimized as there is a trade-off between the lateral resolution and sensitivity of the system [26].

In this paper, we present the design considerations for this specialized ultrasound detector for PA breast imaging. We specify the most important detector output characteristics such as sensitivity and frequency response, and justify the selection of active material and matching layers and their geometries. We iterate between simulation of detector performance, fabrication and experimental characterization of functional models to arrive at an optimized implementation. For computer simulation, we use 1D Krimholtz-Leedom-Matthaei (KLM) models and 3D finite-element (FEM) based models.

\subsection{Design parameters}

\subsubsection{Sensitivity and acceptance angle}

In photoacoustic tomography, the breast is illuminated with short-pulsed laser light and the ultrasound detector scans the object ideally through $360^{\circ}$. A requirement in this geometry is that each US detectors's acceptance angle is wide enough to detect photoacoustic signals generated throughout the entire object for each detector angular position around the object (Figure 3.1). During a backprojection style reconstruction, the coherent signals from all detector angular positions are summed up to form an 


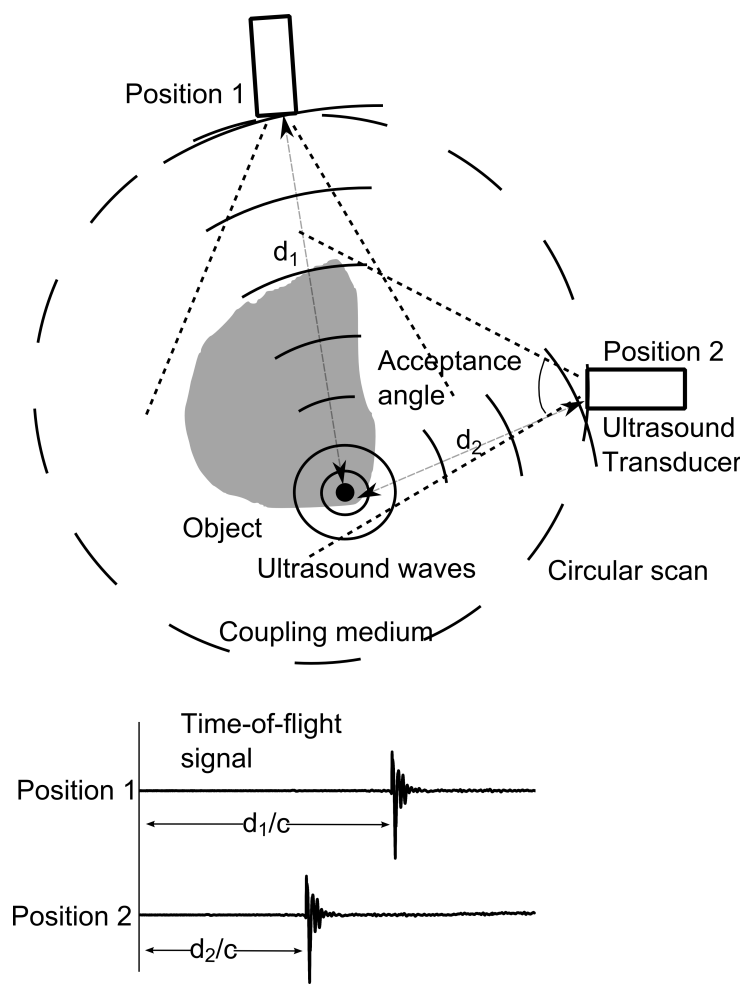

Figure 3.1: Schematics of a 2D photoacoustic tomography system showing the necessity for the acceptance angle to encompass the object for coherent signals detection from all angular position in performing reconstruction.

image of the photoacoustic sources [27]. For a single element square surface detector, the $-6 \mathrm{~dB}$ acceptance angle $(\phi)$ depends on the width $(W)$ and center frequency $\left(f_{0}\right)$ of the detector and can be expressed as [28]:

$$
\phi=2 \sin ^{-1}\left(\frac{3.79}{\mathrm{~kW}}\right)
$$

with $k$ the wavenumber. Equation 1 shows that the acceptance angle decreases as the aperture increases. However a larger surface area is important since for the same piezo element thickness, a lower MDP is attained. This is due to a lower thermal-induced noise due to the higher electrical capacitance associated with larger detectors. To use a sensitive large aperture detector without compromising the acceptance angle, Li et al [29] and Pramanik et al [30] introduced the concept of a negative acoustic lens. Here an acoustic lens enlarges the acceptance angle of a large aperture detector improving the lateral resolution of the PA tomographic system. This provides a solution where 
one can use a large area detector while maintaining a wide acceptance angle.

Based on the above considerations, the choice is made for a large aperture detector with a square surface ( $5 \mathrm{~mm} \times 5 \mathrm{~mm}$ ) with an appropriate lens [31], which will be discussed in a future article. The square shape is chosen for convenience in array development using traditional dicing.

Table 3.1: Properties of the materials used for the detector in 3D FEM simulations. Properties of the PZT material are from reference [40,41], and the properties of the matching and backing layers are from reference [41].

\begin{tabular}{llll}
\hline \hline Properties & Matching layers & Active layer & Backing \\
\hline Material & Electrical conductive epoxy & PZT(CTS 3203HD) & Elastosil \\
Impedance (MRayl) & 6.5 & 38 & 3.4 \\
Density $\left(\mathrm{kg} \mathrm{m}^{-3}\right)$ & 3140 & 7800 & 1870 \\
Longitudinal velocity $\left(\mathrm{m} \mathrm{s}^{-1}\right)$ & 2068 & - & 1818 \\
Shear velocity $\left(\mathrm{m} \mathrm{s}^{-1}\right)$ & 994 & - & 873 \\
Elastic compliance & - & $s_{11}^{E}=1.56, s_{12}^{E}=-0.420$, & - \\
$\left(\mathrm{m}^{2} / \mathrm{Nx} 10^{-11}\right)$ & & $s_{13}^{E}=-0.823, s_{33}^{E}=1.89$, & \\
& & $s_{33}^{E}=3.92, s_{66}^{E}=3.98$ & \\
Piezoelectric strain coefficient & - & $d_{13}=2.95, d_{33}=5.64$, & - \\
$\left(\mathrm{m}^{2} / \mathrm{Nx} 10^{-10}\right)$ & & $d_{15}=5.60$ & \\
Relative permitivity & - & $K_{11}^{T}=2417, K_{33}^{T}=3331$ & - \\
Dielectric loss & - & 0.028 & - \\
Mechanical quality factor & - & 66 & - \\
\hline \hline
\end{tabular}

\subsubsection{Center frequency}

Photoacoustic breast imaging is based on the tissue optical contrast due to vascularization. This is enhanced around a tumor due to the process of angiogenesis [32]. This process is reported to go through two phases separated by the "angiogenic switch". Exponential tumor growth ensues in the second phase (vascular phase), which occurs from tumor sizes of 1-2 $\mathrm{mm}$ in diameter [33]. This indicates that the resolution of our system is preferably to be smaller than $2 \mathrm{~mm}$.

The upper and lower limit of the frequency range of the detector $\left(f_{\max }\right.$ and $\left.f_{\min }(\mathrm{MHz})\right)$ can be estimated knowing the smallest and largest sphere $\left(a_{\min }\right.$ and $a_{\max }(\mathrm{mm}$ in diameter $)$ that are required to be resolved by the system using [19]:

$$
f_{\max }=\frac{3 \nu}{a_{\min }} \& f_{\min }=\frac{0.32 \nu}{a_{\max }}
$$




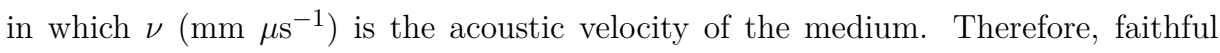
registration of spherical tumors with diameters from $2 \mathrm{~mm}$ to $10 \mathrm{~mm}$ requires an ultrasound detector with frequency bandwidth from $2.25 \mathrm{MHz}$ down to $48 \mathrm{kHz}$. To achieve the required bandwidth, the center frequency of the detector is designed to be $1 \mathrm{MHz}$, while optimizing the bandwidth of the detector to be reasonably broad.

\subsection{Materials and fabricated models}

\subsubsection{Materials}

As mentioned earlier, we prefer PZT detectors due to the superior dielectric constant, lower dielectric loss and higher coupling coefficients [23]. A high sensitivity commercial piezoelectrical material CTS 3203HD (CTS Communications Components, Inc., Albuquerque, NM) is used for the active material due to the higher coupling coefficients compared to other common used commercial PZT materials [34]. A front matching layer is used to improve the sensitivity and bandwidth of the detector, and a back matching layer is used to improve the transmission of ultrasound into the backing layer and thus improve the bandwidth of the detector. The theoretical values for the suitable acoustic impedance of the matching layers $\left(Z_{m}\right)$ can be determined by $[35,38]$ :

$$
Z_{m}=\sqrt{Z_{w} Z_{p}}
$$

where $Z_{w}$ and $Z_{p}$ are the acoustic impedance of water/tissue (or backing) and ferroelectric ceramic, respectively. The material properties of the piezoelectric material, the front matching layer, back matching layer and the backing are carefully chosen based on their acoustic impedances, and all important acoustic properties for these materials are listed in Table 3.1.

\subsubsection{Functional and test models}

\subsubsection{First functional model}

To have a rough assessment of the required characteristics of the detector such as center frequency (around $1 \mathrm{MHz}$ ) and bandwidth $(\geq 80 \%)$, the thicknesses of the active material, front and back matching layer and backing are determined using the KLM model [42](See Sec. 3.4). The first functional model is then manufactured as shown in Figure 3.2(a), with dimensions of each layer listed in Table 3.2. 

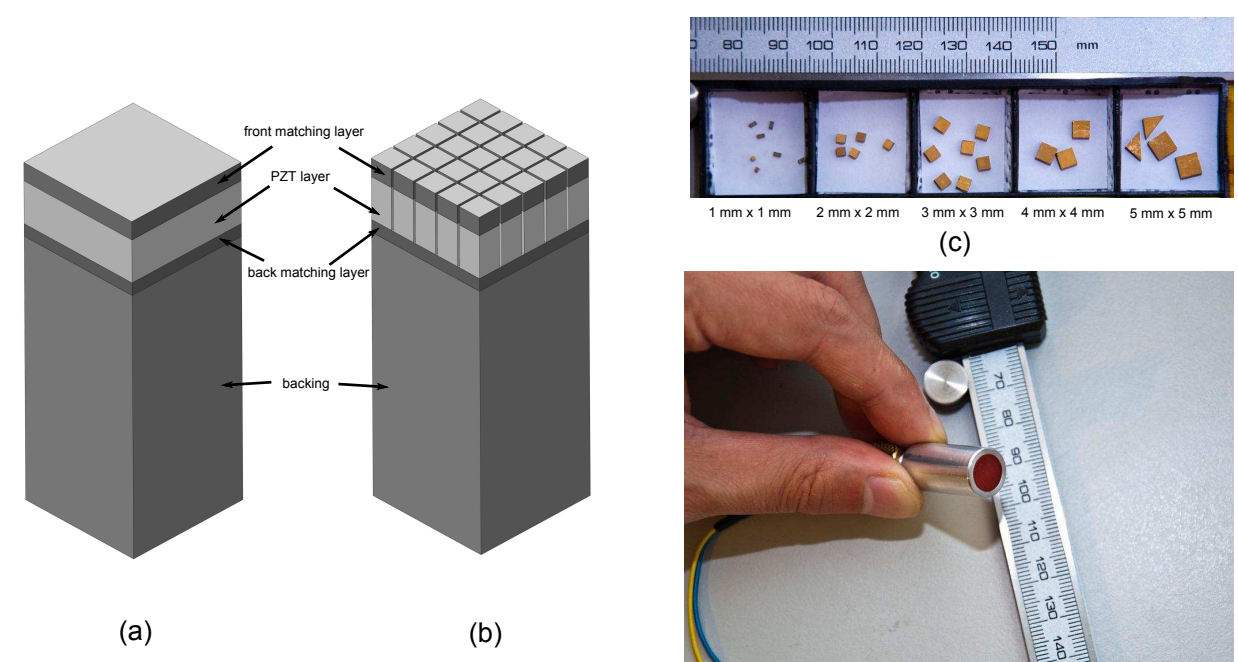

(c)

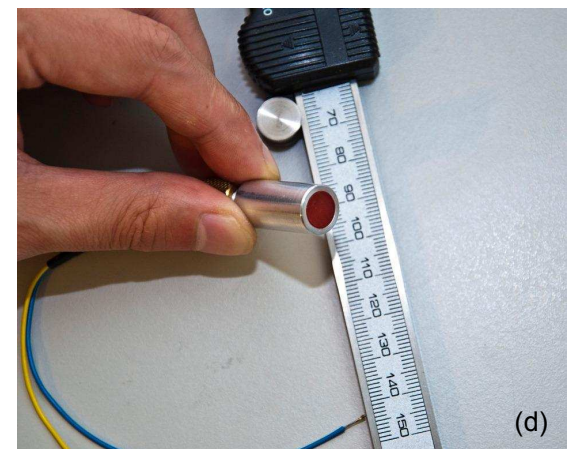

Figure 3.2: (a) Schematic of first functional model. (b) Schematic of subdiced second functional model and final model.(c) Photograph of the bare PZT samples with different lateral dimensions (label below). Two triangular samples are shown in the photograph, however, no related result is reported in this work. (d) Photograph of the final single-element model.

\subsubsection{Test models for minimizing radial resonances}

The large lateral dimensions of the detector result in radial resonances that interfere with the thickness resonance. To reduce the radial resonance of the element, the detector requires to be sub-diced into smaller units, which are acoustically isolated by air kerfs but electrically grouped by common electrodes to form a single composite element [43](Figure 3.2(b)). To identify optimum lateral dimensions of the subdiced unit, five square-shaped, bare ceramics samples (CTS 3203HD) all having a thickness of $1.625 \mathrm{~mm}$ were manufactured as shown in Figure 3.2(c). The different sizes represent the possible subdicing sizes applied to the $5 \mathrm{~mm} \times 5 \mathrm{~mm}$ element to suppress the radial resonances. 3D finite-element method based models for these PZT samples are built using PZFlex (Weidlinger Associates Inc, Los Altos, CA) (See Sec. IV) to study the effect of sub-dicing, and to design the size of the sub-diced small unit.

\subsubsection{Second functional model}

This version results from the experiences with the first functional model and the test models above, and attempts to minimize interference from lateral resonances. For this, the first functional model is sub-diced into $0.9 \mathrm{~mm} \times 0.9 \mathrm{~mm}$ units using a 100 
Table 3.2: Layer thicknesses of functional models (Figure 3.2(a) and (b)), each layer has a $5 \mathrm{~mm} \times 5 \mathrm{~mm}$ square-shape surface.

\begin{tabular}{llll}
\hline \hline Layer description & Material & $\begin{array}{l}1^{\text {st }} \text { and } 2^{\text {nd }} \text { functional models } \\
\text { thickness }(\mathrm{mm})\end{array}$ & $\begin{array}{l}\text { Final model } \\
\text { thickness }(\mathrm{mm})\end{array}$ \\
\hline Front matching layer & Electrical conductive epoxy & 0.590 & 0.700 \\
Active layer & CTS 3203HD & 1.625 & 1.625 \\
Back matching layer & Electrical conductive epoxy & 0.788 & 0.480 \\
Backing & Elastosil & 10 & 10 \\
\hline \hline
\end{tabular}

$\mu \mathrm{m}$ dicing saw. Only the front matching layer and active layer are sub-diced. The 25 units are acoustically isolated by air kerfs, but electrically grouped by two electrodes (Figure 3.2(d)). The dimensions of each layer are listed in Table 3.2.

\subsubsection{Final model}

With the second functional model above found suitable to minimize the presence of lateral resonance in the frequency range of interest, we studied the variation of passive layers dimensions on the frequency response using 3D FEM models. To increase the bandwidth of the detector, the thicknesses of the front and back matching layer need to be optimized. Three-dimensional FEM models are simulated to estimate the pulseecho signals of the detectors, from which the frequency responses of the detectors are calculated to arrive at an optimized implementation. We manufactured a final model according to the geometrical parameters achieved as shown in Figure 3.2(b,d). The dimensions of each layer are listed in Table 3.2.

\subsection{Numerical and experimental methods}

\subsubsection{Simulation methods used}

\subsubsection{1D KLM model}

An equivalent circuit based 1D KLM model [42] is used to obtain a rough assessment of the required detector performances such as center frequency and bandwidth.

\subsubsection{3D FEM model}

For a more accurate estimation, coupled partial differential equations for piezoelectricity and acoustic wave propagation through passive material layers of the detector and coupling medium require to be solved [43]. This can be appropriately done by 
using 3D FEM models. In this study, 3D FEM models are built using PZFlex (version 3.0, Weidlinger Associates Inc, Los Altos, CA) as shown in Figure 3.2(a) and (b). The finite element size is defined as $1 / 30$ of the ultrasound wavelength at the designed transducer center frequency $(1 \mathrm{MHz})$. The roughly estimated results from KLM models are used as the starting point for the subsequent iterations between 3D simulations of detector performance and experimental characterization of functional models. The material properties used for FEM simulation are listed in Table 3.1.

\subsubsection{Detector characterization methods}

\subsubsection{Electrical impedance}

In order to ascertain the resonance characteristics, and to study the behaviour of the detector in an electrical measurement chain, the complex electrical impedance is measured using an impedance analyzer (4194A/B, Hewlett-Packard, Palo Alto, California).

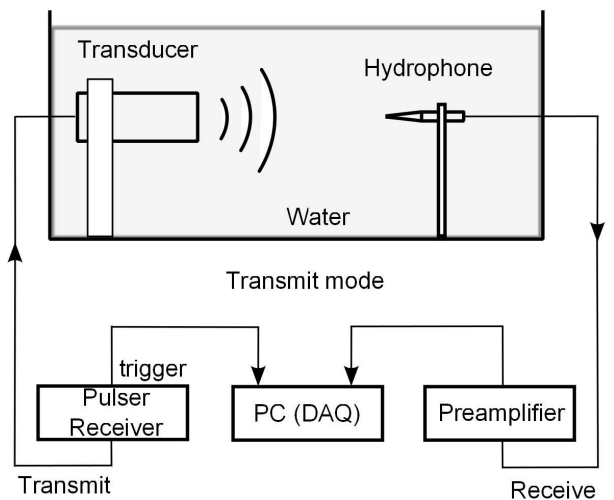

(a)

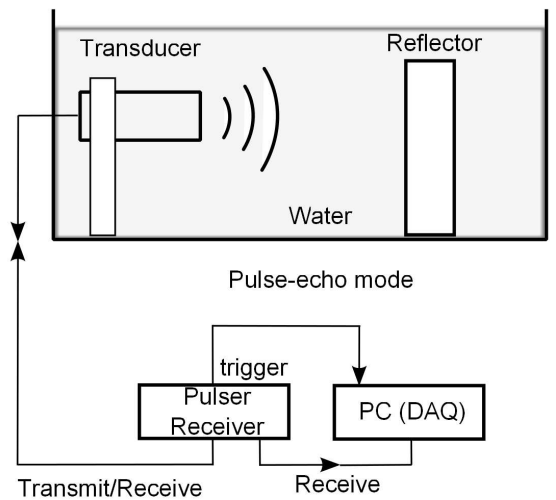

(b)

Figure 3.3: Schematics of the setups for detector frequency response measurements. (a) Transmit mode. (b) Pulse-echo mode.

\subsubsection{Acoustic frequency response}

Two methods were used to measure the frequency response: (1) a transmit mode using a hydrophone, and (2) a pulse-echo mode.

In method 1 (Figure 3.3(a)), the detector immersed in a demineralized water bath 
is driven by a broadband ultrasonic pulser/receiver (Panametrics 5077PR), and the generated pressure is probed in the far-field using a calibrated broadband needle hydrophone (0.2 mm diameter, bandwidth upto $10 \mathrm{MHz}$, Precision Acoustic Ltd., Dorchester, UK) with a known receiving transfer function $H_{0}(f)$. The frequency response of the detector $(H(f))$ is then calculated by:

$$
H(f)=\frac{\operatorname{FFT}\{P(t)\}}{H_{0}(f)}
$$

Method 2 (Figure 3.3 (b)) uses the detector in pulse-echo mode, driven by the pulser-receiver. A stainless steel plate is placed in the far-field of the detector as an acoustic reflector, and the reflected signal (pulse-echo) is measured by the detector. According to the principle of reciprocity for a piezoelectric detector [44,45], the frequency response of an ultrasound transducer is the same when used as receiver or transmitter. Thus the frequency response of the detector is calculated by the square root of the FFT of the measured pulse-echo signal.

\subsubsection{Directivity}

The directivity of the detector is measured in transmit mode. The detector is mounted in a demineralized water bath and driven by the broadband pulser/receiver. The calibrated needle hydrophone is rotated in the far-field $(60 \mathrm{~mm})$ centered on the detector element to probe the emitted ultrasound field through $180^{\circ}$. For each scanning position, the peak-to-peak value is determined from the ultrasound pulse recorded by the hydrophone and plotted as a function of scanning angle, giving the directivity of the transducer at its center frequency.

\subsubsection{Sensitivity and minimum detectable pressure}

We measured these parameters using a modified substitution method [9] as outlined. We insonify the detector element using an ultrasound transmitter. The pressure incident on the detector element is progressively reduced, by reducing the voltage input to the transmitter. At each incident pressure, the voltage output of the detector is noted while averaging multiple times. This is continued till the lowest input possible. This detector output is defined as the signal. In a next step we note the noise voltage on the detector element without averaging and with no pressure incident on the element. The signal is plotted against pressure input and the trend is extended to intersect the voltage noise floor. This intersection point signifies $\mathrm{SNR}=1$, and the pressure at which this signal is expected/obtained is the minimum detectable pressure. 
In the experiments we used a $1 \mathrm{MHz}$ unfocused broadband transducer (V303, Panametrics) as a transmitter. Signals were processed by a prototype low noise preamplifier based on Analog Devices ADA4896-2 as the analog front-end and acquired using a high-speed digitizer NI-5752 (National Instruments). A calibrated needle hydrophone was used to ascertain the transfer function of the transmitter and convert the input voltage of the transmitter to known pressure that insonified the detector element.

\subsubsection{Imaging quality simulation}

To study the resolution and visibility of objects with a photoacoustic tomography (PAT) system employing the optimized detector, and comparing performances using detectors described in the literature, numerical simulations are performed using kwave Matlab Toolbox [36].

For the forward simulation, a 2D initial pressure distribution map (1024 x 1024 grid, $20 \mathrm{~cm} \times 20 \mathrm{~cm}$ size) is assigned in a 2D tomographic configuration. Three discshaped objects with diameters of $10 \mathrm{~mm}, 2 \mathrm{~mm}$ and $0.5 \mathrm{~mm}$ are located in the center region of the map. Homogenous initial pressure (value 1) is assigned to the objects, a pressure value of zero is given to the rest of the map. Homogenous acoustic properties are assigned to the medium (speed of sound: $1500 \mathrm{~m} / \mathrm{s}$, acoustic attenuation: 0 , density: $1000 \mathrm{~kg} / \mathrm{m}^{3}$.) Those pressures propagating outward are detected by a $5 \mathrm{~mm}$ detector rotating around the objects with radius of $10 \mathrm{~cm}$ covering $360^{\circ}$ with step size of $2^{\circ}$. The time-domain photoacoustic signals are averaged over the surface of the detector at each detection position and saved for further processing.

Three detectors are simulated, from:

(1) The final model of this work.

(2) The Kruger group (1 MHz center frequency and 100\% bandwidth) [37].

(3) The Oraevsky group (1.25 MHz center frequency and a bandwidth approaching $200 \%$, derived from $[11,19])$.

For (1), signals received by the detector are convolved with measured impulse response of the final model. For (2) and (3), the received signals are filtered using Gaussian bandpass filters with the corresponding center frequency and bandwidth. Correspondingly, three images are reconstructed by time-reversal of the processed signals. 


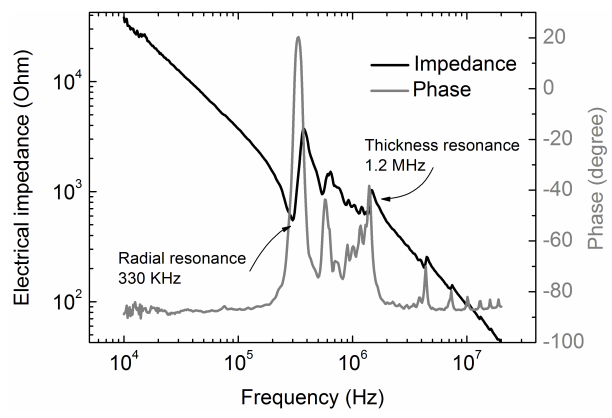

(a)

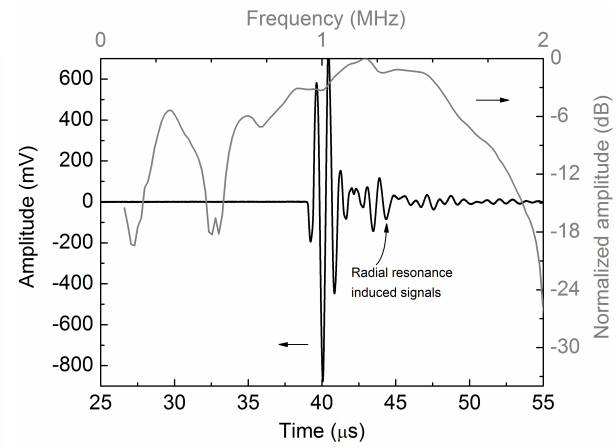

(b)

Figure 3.4: First functional model performance. (a) Electrical impedance of the first functional model measured with water load. (b) Measured transmission impulse response and frequency transfer function of the first functional model using a hydrophone. (time domain left axis, frequency domain right axis).

\subsection{Results}

\subsubsection{First functional model performance}

Figure 3.4(a) shows the measured electrical impedance of the first functional model. A fundamental thickness resonance at $1.2 \mathrm{MHz}$ together with a series of harmonic thickness resonances at higher frequencies, and a strong radial resonance at $330 \mathrm{kHz}$ together with a second harmonic radial resonance at around $700 \mathrm{kHz}$, can be observed. The fundamental thickness resonance corresponds to the designed thickness of the active layer $(1.625 \mathrm{~mm})$ and the low frequency radial resonance arises due to the large lateral dimensions of the active layer (5 mm x $5 \mathrm{~mm}$ ) [39].

Figure 3.4(b) shows the measured frequency response of the first functional model using method 1 described above. Two peaks can be observed in the frequency domain response: a $1.2 \mathrm{MHz}$ peak caused by the thickness resonance, and a peak at $330 \mathrm{KHz}$ caused by the radial resonance. Both peaks match with the measured electrical impedance peaks (Figure 3.4(a)). The first functional model has a center frequency of $1.2 \mathrm{MHz}$ (maximum peak), with a $-6 \mathrm{~dB}$ bandwidth of $0.8 \mathrm{MHz}$. The fractional bandwidth is $67 \%$. Due to the radial resonance, a secondary pulse (reverberation-like signal) after the main pulse is visible in the time domain signal, which is manifested as a passband ripple in the frequency domain.

The measured strong lateral resonance of the first functional model is not desirable. First the detector sensitivity is reduced by the lateral mode resonance [43]. Second, 
strong lateral mode causes additional reverberation-like signals (Figure 3.4(b)), which will adversely influence the image quality of the system [46].
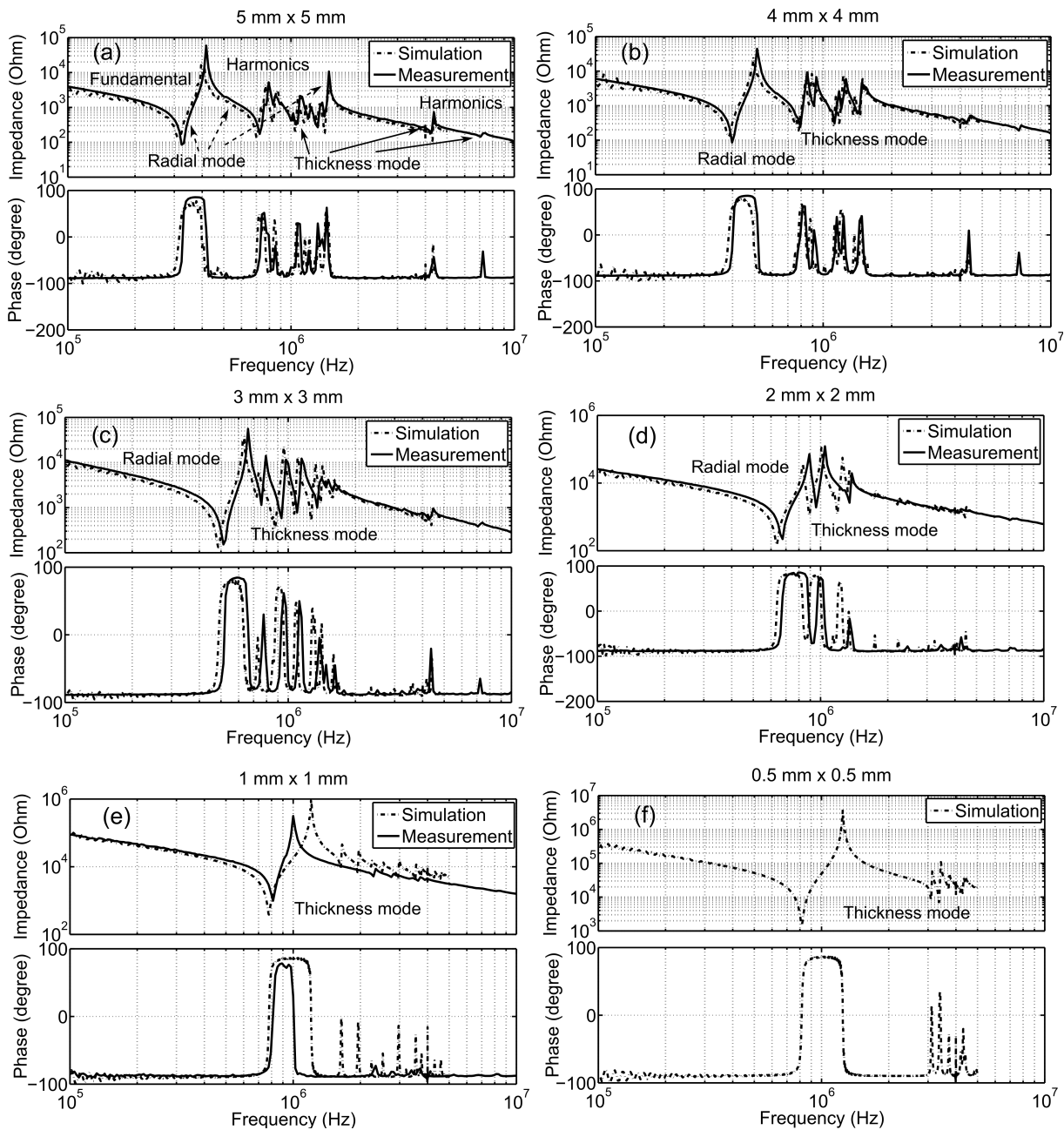

Figure 3.5: Test samples of PZT: measured and simulated electrical impedance in air with lateral dimensions (a) $5 \mathrm{~mm} \times 5 \mathrm{~mm}$; (b) $4 \mathrm{~mm} \times 4 \mathrm{~mm}$; (c) $3 \mathrm{~mm} \times 3 \mathrm{~mm}$; (d) $2 \mathrm{~mm} \times 2 \mathrm{~mm}$; (e) $1 \mathrm{~mm} \mathrm{x}$ $1 \mathrm{~mm}$ and (f) $0.5 \mathrm{~mm} \times 0.5 \mathrm{~mm}$. No measured impedance available for $0.5 \mathrm{~mm} \times 0.5 \mathrm{~mm}$ PZT due to the practical limitations in manufacturing and measuring. 


\subsubsection{Test models performances}

Figure 3.5 shows the measured and simulated electrical impedance of the bare piezoceramic samples with thickness of $1.625 \mathrm{~mm}$ and lateral dimensions ranging from 5 $\mathrm{mm} \times 5 \mathrm{~mm}$ down to $0.5 \mathrm{~mm} \times 0.5 \mathrm{~mm}$. In general, FEM simulations exhibit good agreement with measurements (due to the manufacturing and measurement difficulties for the smaller bare ceramics, electrical impedance measurements for the $0.5 \mathrm{~mm}$ x $0.5 \mathrm{~mm}$ ceramic is not available). The fundamental thickness resonance peak for all samples is always located around $1 \mathrm{MHz}$, while the lateral resonance frequency peaks move towards higher frequencies as the lateral dimensions decrease. For lateral dimensions of $1 \mathrm{~mm} \times 1 \mathrm{~mm}$ the radial resonance mode is suppressed. Further reduction in lateral dimensions to $0.5 \mathrm{~mm} \times 0.5 \mathrm{~mm}$ does not significantly change the amplitude and location of the thickness resonance peak (Figure 3.5(e) and (f)). Since more active material is lost with finer subdicing, leading to detection sensitivity loss, we prefer $1 \mathrm{~mm}$ x $1 \mathrm{~mm}$ as the final choice.

\subsubsection{Second functional model}

The measured electrical impedance of the second functional model in water is compared with 3D FEM simulation in Figure 3.6(a). Both simulation and measurement show that the lateral resonance is not visible in the electrical impedance curves as expected. The fundamental thickness resonance is located at around $1 \mathrm{MHz}$.

The frequency response of the detector measured using method 2 compared with 3D FEM simulations is shown in Figure 3.6(c). Both measurement and simulation shows the detector has a center frequency of $0.9 \mathrm{MHz}$, however with a $-6 \mathrm{~dB}$ fractional bandwidth of $48 \%$, which is low for photoacoustic applications.

\subsubsection{Final model}

The starting point for the thickness optimization is based on the 1-D KLM model [42]; this gives the optimized front matching layer thickness $\left(t_{M L-F}\right)$ of $0.58 \mathrm{~mm}$ and back matching layer thickness $\left(t_{M L-B}\right)$ of $0.54 \mathrm{~mm}$ as used for the first functional model (Figure 3.4(b)). In the first phase of the optimization, $t_{M L-B}$ is kept constant $(0.54 \mathrm{~mm})$, and $t_{M L-F}$ is tuned to obtain the largest bandwidth (Figure 3.7(a)-(c)). The optimum $t_{M L-F}$ value is then determined $(0.70 \mathrm{~mm})$. The second phase is to keep the optimized $t_{M L-F}$ constant, and to optimized $t_{M L-B}$. After the two phase optimization, the optimum $t_{M L-F}$ and $t_{M L-B}$ are determined (Figure $3.7(\mathrm{~d})$ ). 

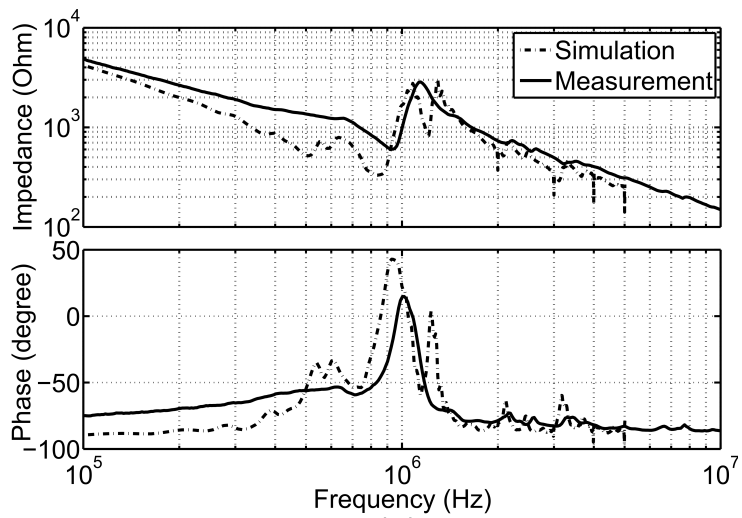

(a)

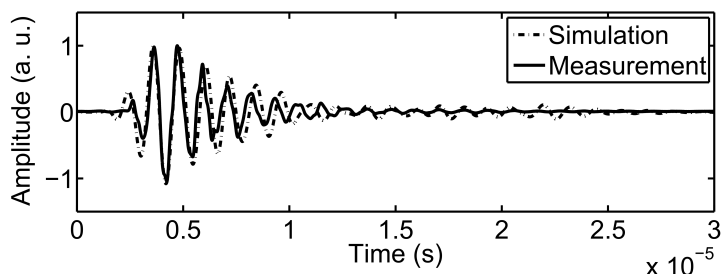

(b)

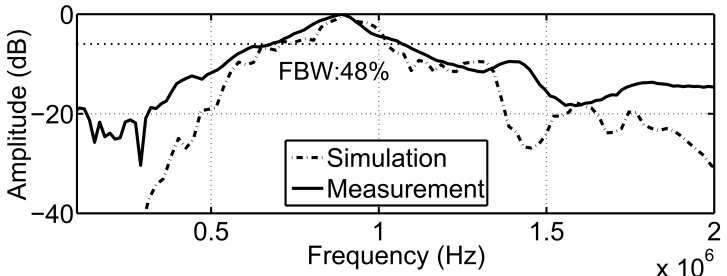

(c)

Figure 3.6: Second functional model performance: (a) Measured and simulated electrical impedance in water. (b) Measured and simulated pulse-echo ultrasound signal. The reflector is placed in the far-field of the detector. The time delay is removed. (c) Measured and simulated frequency response.

The simulation results in Figure 3.7 show that the bandwidth of the detector is increased from around 50\% (Figure 3.7(a)) to around 70\% (Figure 3.7(c)) by optimizing the front matching layer. Further optimization of the back matching layer increases the bandwidth of the detector to more than $80 \%$ (Figure 3.7(d)), which is generally high for a PZT detector with single front matching layer [47]. 


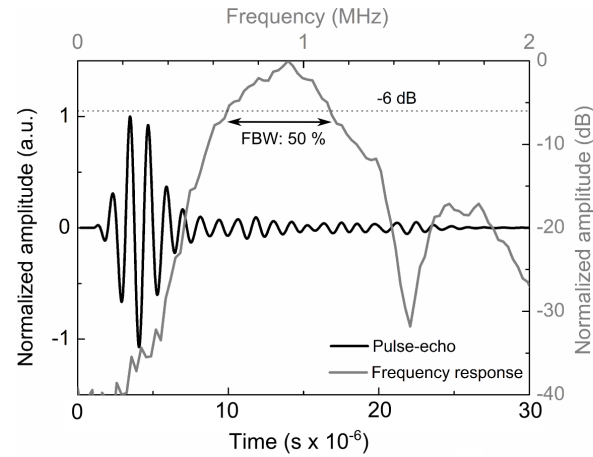

(a)

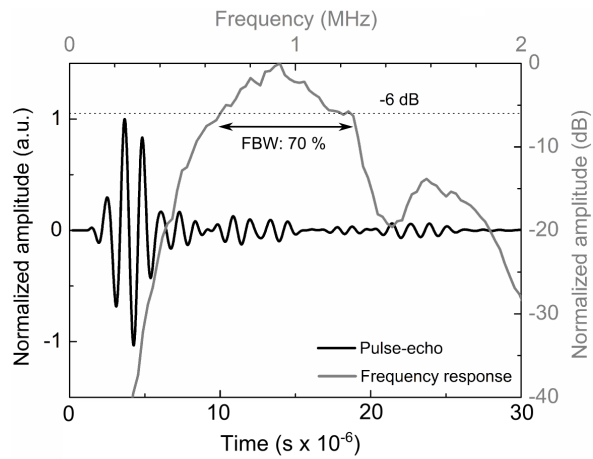

(c)

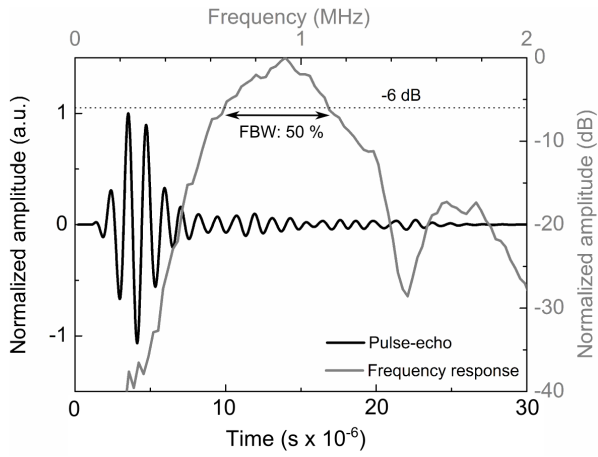

(b)

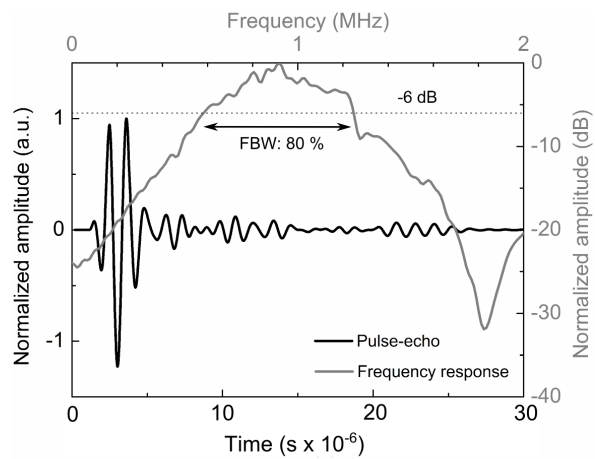

(d)

Figure 3.7: Towards optimized final model: simulated pulse-echo signal and the frequency response of the sub-diced detector with different front- and back- matching layer thicknesses. (a) $t_{M L-F}$ : $0.58 \mathrm{~mm}, t_{M L-B}: 0.54 \mathrm{~mm}$. (b) $t_{M L-F}: 0.55 \mathrm{~mm}, t_{M L-B}: 0.54 \mathrm{~mm}$. (c) $t_{M L-F}: 0.70 \mathrm{~mm}$, $t_{M L-B}: 0.54 \mathrm{~mm}$. (d) $t_{M L-F}: 0.70 \mathrm{~mm}, t_{M L-B}: 0.48 \mathrm{~mm}$.

\subsubsection{Frequency response}

The measured frequency response and time domain signal in the farfield $(60 \mathrm{~mm}$ away) show good agreement with simulation results in Figures 3.8(a) and 3.8(b). Compared to the ultrasound pulse for the first functional model (Figure 3.4(b)), the optimized pulse is considerably shorter in length. The measured center frequency is $1 \mathrm{MHz}$, which is slightly higher than the model predicted $0.9 \mathrm{MHz}$ (Figure 3.8(b)). The measured $-6 \mathrm{~dB}$ bandwidth ranges from $0.4 \mathrm{MHz}$ to $1.25 \mathrm{MHz}$, which is also slightly larger than the simulation result $(0.55 \mathrm{MHz}-1.25 \mathrm{MHz})$, while the fractional bandwidths are similar for both $(80 \%)$. 


\subsubsection{Directivity}

Figure 3.8(c) shows the measured directivity of the final model. The measurement results compare well with simulations, both showing a $-6 \mathrm{~dB}$ acceptance angle around $20^{\circ}$ at $1 \mathrm{MHz}$. When a $5 \mathrm{~mm}$ diameter hemispherical acoustic lens is placed on top of the detector, the directivity angle of the detector is expected to be enlarged to around $60^{\circ}[31]$.

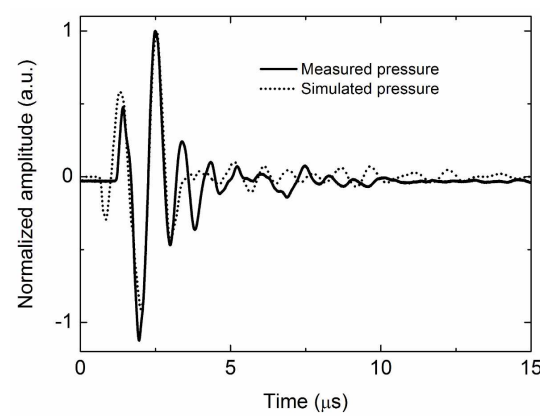

(a)

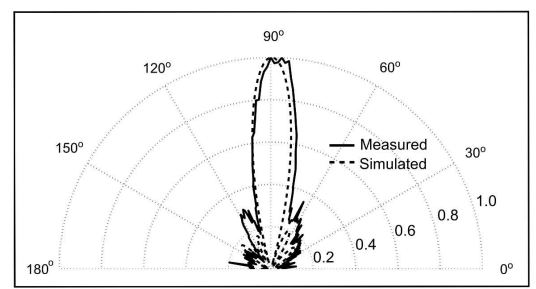

(c)

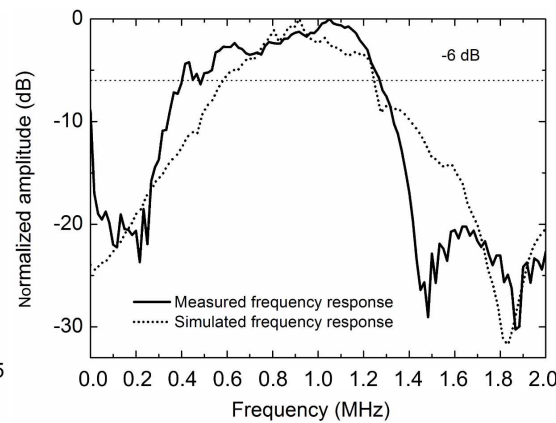

(b)

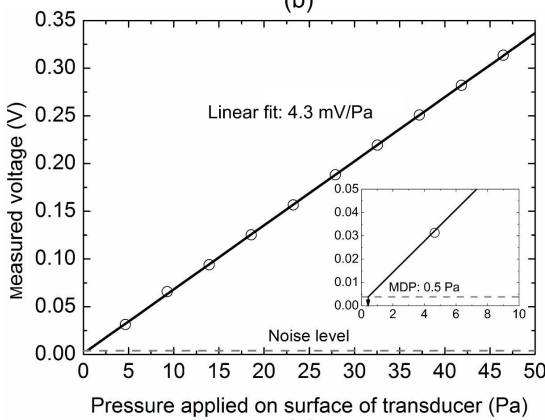

(d)

Figure 3.8: Final model performance: (a) Measured and simulated far-field pulse of the final model time-shifted to origin. For the measurement, the pulse is probed using a calibrated broadband needle hydrophone in the far-field at distance $60 \mathrm{~mm}$, on center axis. (b) Measured and simulated frequency response of the detector. (c) Measured and simulated directional sensitivity. (d) Measured sensitivity and minimum detectable pressure (MDP).

\subsubsection{Sensitivity}

The end-of-cable minimum detectable pressure of the detector-electronics was estimated to be $0.5 \mathrm{~Pa}$. As seen the Figure 3.8(d)) the signal trend is extrapolated to intersect the noise floor to provide the point at which $\mathrm{SNR}=1$. The corresponding 


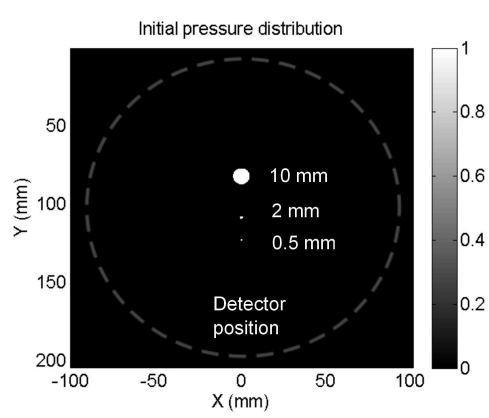

(a)

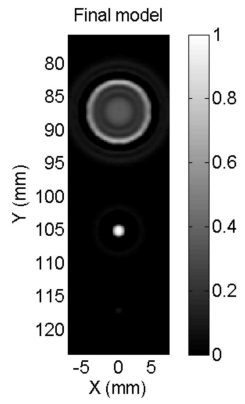

(b)

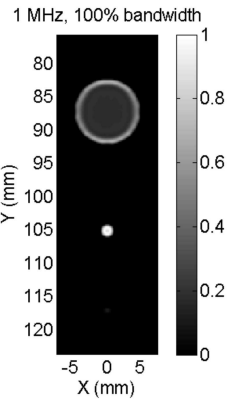

(c)

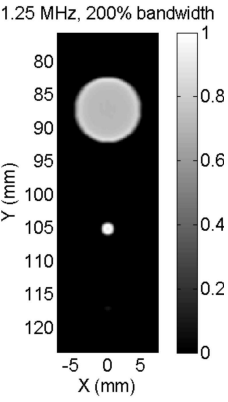

(d)

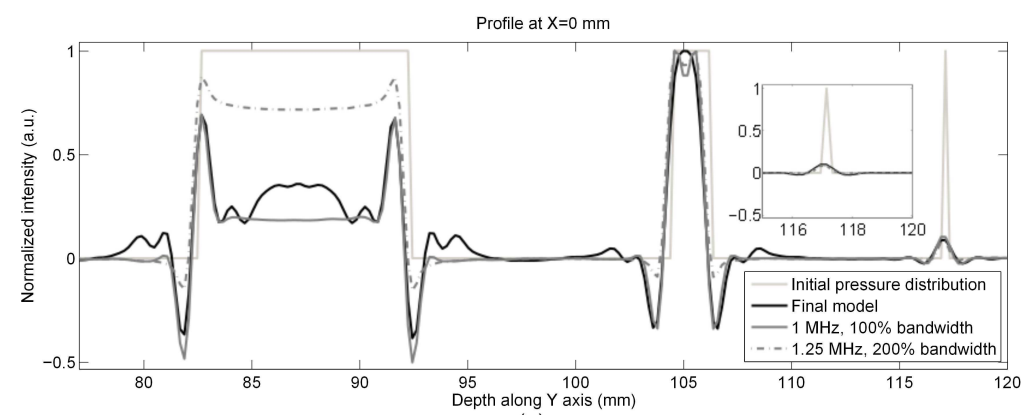

(e)

Figure 3.9: (a) Initial pressure distribution used in the forward simulation. The gray dashed circle indicates the detector scanning positions. (b) Reconstructed image using signals detected by the final model, (c) by the transducer with $1 \mathrm{MHz}$ center frequency and 100\% fractional bandwidth (Kruger et al 1999 [37]) and (d) by the transducer with $1.25 \mathrm{MHz}$ center frequency and $200 \%$ fractional bandwidth (Andreev et al 2003 [19] and Ermilov et al 2009 [11]). (e)Profiles at position X=0 mm from the initial pressure distribution in (a) and reconstructed images from (b), (c) and (d).

pressure can be read off on the x-axis.

\subsubsection{Imaging quality}

The reconstructed images from the three cases are shown: for our detector in Figure 3.9(b), Kruger system [37] in Figure 3.9(c), and the Oraevsky system [11,19] in Figure 3.9(d). It is clearly shown that Oraevsky detector, due to the superior bandwidth of the detector, possesses excellent image quality and faithfully recovers the original initial pressure distribution. Ours and Kruger's system faithfully recover the $2 \mathrm{~mm}$ object. The $10 \mathrm{~mm}$ object is visualized with edge enhancement. The resolution of three systems is around 1-2 mm, which can be estimated from the reconstruction of the sub-resolution $0.5 \mathrm{~mm}$ object (see profiles in Figure 3.9(e)). 


\subsection{Discussion}

The final optimized single-element detector has a fractional bandwidth of $80 \%$, which matches with the model prediction. The center frequency of the detector is around $1 \mathrm{MHz}$ and $-6 \mathrm{~dB}$ bandwidth is from $0.40 \mathrm{MHz}$ to $1.25 \mathrm{MHz}$. From simulation of system resolution and image quality (Figure 3.9(b)), it is found that the object with diameter of $2 \mathrm{~mm}$ can be faithfully recovered, and the $10 \mathrm{~mm}$ object can be visualized with acceptable distortions (edge enhancement and ring-shaped artifacts). The resolution of our system reaches the designed goal of 1-2 mm (Figure 3.9(e)). The simulations are performed with a high optical contrast of the object, which gives large signal-to-noise ratios. This condition is applicable for a sensitive detector like ours: frequencies beyond the $-6 \mathrm{~dB}$ bandwidth of the detector will still contribute to signal detection, which makes the resolution of our system better than the value calculated from Eq.3.2 [19] taking only -6 dB bandwidth of the detector into account. The resolution metrics used via k-wave simulations do not necessarily line up with those from Eq.3.2 because Eq.3.2 is derived only from the frequency contents of the photoacoustic signals generated from objects with varying sizes. This approach gives an indication for designing the ultrasound detectors, while imaging approach via $\mathrm{k}$-wave simulation is suitable to characterize the overall system resolution. This indicates that the present detector is suitable to detect tumors during the beginning of the vascular phase. The image quality can be further improved by enlarging the bandwidth of the detector using two or more front matching layers [47]. However, a larger bandwidth reduces the sensitivity of the detector, and increases complexity and expense during transducer design and development and in final production.

The directivity angle of the transducer is around $20^{\circ}$, which can be increased to around $60^{\circ}$ using an acoustic lens. For simplicity, considering the breast as a hemisphere with diameter of $10 \mathrm{~cm}$, the transducer with directivity angle of $60^{\circ}$ is required to be placed only $5 \mathrm{~cm}$ away from the breast. This distance is suitable for the application.

We have consolidated the most important output characteristics of the optimized ultrasound detector in Table 3.3, in row 7 described as the PAM-II system. We have also provided specifications of various ultrasound detectors described in literature for comparison. The MDP value of our detector is $0.5 \mathrm{~Pa}$, which is 160 times lower than for the detector used in the first generation of the Twente Photoacoustic Mammoscope previously developed in our group [20]. To the best of our knowledge, this is the lowest measured MDP reported for a detector in photoacoustic breast imaging (Table 3.3). 
Table 3.3: List of US detectors used by different groups in the photoacoustic (thermoacoustic) systems for breast imaging.

\begin{tabular}{|c|c|c|c|c|c|c|c|}
\hline Groups & System & $\begin{array}{l}\text { Detector } \\
\text { elements }\end{array}$ & $\begin{array}{l}\text { Element } \\
\text { geometry } \\
(\mathrm{mm})\end{array}$ & $\begin{array}{l}\text { Active } \\
\text { material }\end{array}$ & $\begin{array}{l}\text { Center } \\
\text { frequency } \\
(\mathrm{MHz})\end{array}$ & $\mathrm{BW}$ & $\begin{array}{l}\mathrm{MDP} \\
(\mathrm{Pa})\end{array}$ \\
\hline (1) Kruger group & $\mathrm{TA}[17]$ & 64 & $\varnothing 13$ & 1-3 composite $\mathrm{PZT}^{*}$ & 1 & $70 \% *$ & - \\
\hline (2) Kruger group & $\mathrm{PA}[10]$ & 128 & $\varnothing 3$ & 1-3 composite $\mathrm{PZT}^{*}$ & 5 & $70 \% *$ & - \\
\hline (3) Wang group & $\mathrm{TA} \& \mathrm{PA}[12]$ & 1 & $\varnothing 13 / 6$ & Piezocomposite $^{\dagger}$ & 2.25 & $60-120 \% \dagger$ & - \\
\hline (4) Oraevsky group & LOIS-64 [11] & 64 & $20 \times 3$ & PVDF & 1.25 & $170 \% \S$ & $* *$ \\
\hline (5) Kyoto University & PAM $[48]$ & 345 & $2 \times 2$ & Piezocomposite & 1 & $\geq 70 \%$ & - \\
\hline (6) University of Twente & PAM [24] & 590 & $2 \times 2$ & PVDF & 1 & $130 \%$ & 80 \\
\hline (7) University of Twente & PAM-II ${ }^{\ddagger}$ & 1 & $5 \times 5$ & CTS 3203HD & 1 & $80 \%$ & 0.5 \\
\hline
\end{tabular}

* R. Kruger (2012), private communication.

$\dagger$ L. V. Wang and M. Pramanik (2012), private communication.

$\S$ A. A. Oraevsky (2012), private communication.

** Various values are reported in literature including measured [21], estimated [19,22], and a measured MDP of 1.8

Pa from A. A. Oraevsky (2012), private communication.

¥ Planned.

The total noise performance of the system could possibly further be improved with better electrical shielding and grounding.

The bandwidth of our detector is $80 \%$, which is slightly broader than the detectors used by Kruger group and Kyoto University in their breast imagers (See Table 3.3). The detector from the Oraevsky group has an ultrabroad bandwidth approaching $200 \%$ (estimated from Refs. [11,19]), which provides impressive image quality as shown in Figure 3.9(d). The image quality of our detector can be improved with the use of a deconvolution operation as is performed by several groups [49-52] to compensate for finite bandwidths effects. Further breast tumors are known to be heterogeneous with a scattered distribution of absorbing regions. In such a case our detector is eminently suited to faithfully image a collection of small absorbing structures, which makes up the tumor mass. We are aware that there is room for improvement, nevertheless our design strategy has resulted in a detector with acceptable bandwidth which is well suited for sensitive clinical breast imaging due to its unprecedented high sensitivity.

Detector arrays will be manufactured based on the single-element detector described in this work for use in the second version of the Twente Photoacoustic Mammoscope (PAM-II) [9]. 


\subsection{Conclusion}

A single-element PZT, large-aperture, sensitive and broadband detector is designed and developed for photoacoustic tomography of the breast. Finite-element based models are used to optimize an initial detector to reduce the radial resonance and optimize the bandwidth of the detector. The center frequency and $-6 \mathrm{~dB}$ fractional bandwidth of the optimized detector are $1 \mathrm{MHz}$ and around $80 \%$, respectively. The minimum detectable pressure is $0.5 \mathrm{~Pa}$, which is more than two orders of magnitude lower than in our first generation photoacoustic breast imaging system and among the lowest reported in the literature. Detector arrays will be manufactured based on the design of this single-element detector for use in the second version of the Twente Photoacoustic Mammoscope. 


\section{Bibliography}

[1] D. Razansky, M. Distel, C. Vinegoni, R. Ma, N. Perrimon, R. W. Köster and V. Ntziachristos "Multispectral opto-acoustic tomography of deepseated fluorescent protein it in vivo," Nat. Photonics 3, 412-417 (2009). (doi:10.1038/nphoton.2009.98)

[2] L. V. Wang and S. Hu, "Photoacoustic tomography: in vivo imaging from organelles to organs", Science 335,(2012). (doi:10.1126/science.1216210)

[3] X. Wang, Y. Pang, G. Ku, X. Xie, G. Stoica, L. V. Wang, "Noninvasive laserinduced photoacoustic tomography for structural and functional in vivo imaging of the brain", Nat. Photonics 21, no.7, 803-806 (2003).

[4] J. Jose, S. Manohar, R. G. M. Kolkman, W. Steenbergen and T. G. van Leeuwen, "Imaging of tumor vasculature using Twente photoacoustic systems," J. Biophoton 2, 701-717 (2009). (doi: 10.1002/jbio.200910025)

[5] P. Beard, "Biomedical photoacoustic imaging," Interface Focus 1, 602-631 (2011). (doi:10.1098/?rsfs.2011.0028)

[6] J. Gamelin, A. Maurudis, A. Aguirre, F. Huang, P. Guo, L. V. Wang, and Q. Zhu, "A real-time photoacoustic tomography system for small animals," Opt. Express 17(13), 10489-10498 (2009).

[7] B. J. Tromberg, N. Shah, R. Lanning, A. Cerrusi, J. Espinoza, T. Pham, L. Svaasand, and J. Butler, "Non invasive in vivo characterization of breast tumors using photon migration spectroscopy," Neoplasia 2, 26Ü40 (2000).

[8] B. Tromberg, B. W. Pogue, K. D. Paulsen, A. G. Yodh, D. A. Boas and A. E. Cerussi, "Assessing the future of diffuse optical imaging technologies for breast cancer management," Med. Phys. 35, 2443-2451 (2008). (doi:10.1118/1.2919078) 
[9] D. Piras, W. Xia, W. Steenbergen, T. G. van Leeuwen and S. Manohar, "Photoacoustic imaging of the breast using the Twente Photoacoustic Mammoscope: Present status and future perspectives," IEEE J. Sel. Topic Quantum. Electron. 16, 730-739 (2010). (doi:10.1109/JSTQE.2009.2034870)

[10] R. A. Kruger, R. B. Lam, D. R. Reinecke, S. P. D. Rio and R. P. Doyle, "Photoacoustic angiography of the breast," Med. Phys. 37, 6096-6100 (2010). (doi:10.1118/1.3497677)

[11] S. A. Ermilov, T. Khamapirad, A. Conjusteau, M. H. Leonard, R. Lacewell, K. Mehta, T. Miller and A. A. Oraevsky, "Laser optoacoustic imaging system for detection of breast cancer," J. Biomed. Opt. 14, 024007 (2009). (doi: http://dx.doi.org/10.1117/1.3086616)

[12] M. Pramanik, G. Ku, C. Li and L. V. Wang, "Design and evaluation of a novel breast cancer detection system combining both thermoacoustic (TA) and photoacoustic (PA) tomography," Med. Phys. 35, 2218-2223 (2008). (doi: $10.1118 / 1.2911157)$

[13] T. Kitai, M. Torii, T. Sugie, S. Kanao, Y. Mikami, T. Shiina and M. Toi, "Photoacoustic mammography: initial clinical results," Breast Cancer, (2012). (doi: 10.1007/s12282-012-0363-0)

[14] M. Heijblom, D. Piras, W. Xia, J.C.G. van Hespen, J.M. Klaase, F.M. van den Engh, T.G. van Leeuwen, W. Steenbergen, and S. Manohar, "Visualizing breast cancer using the Twente photoacoustic mammoscope: What do we learn from twelve new patient measurements?," Opt. Express 20, 11582-11597 (2012). (doi: http://dx.doi.org/10.1364/OE.20.011582)

[15] M. I. Khan and G. J. Diebold, "The photoacoustic effect generated by an isotropic solid sphere," Ultrasonics 33, 265-269 (1995). (doi: http://dx.doi.org/10.1016/0041-624X(95)00034-Z)

[16] T. J. Allen and P. C. Beard, "Optimising the detection parameters for deep-tissue photoacoustic imaging," Proc. SPIE 8223, 82230p (2012). (doi: http://dx.doi.org/10.1117/12.908813)

[17] R. A. Kruger, K. D. Miller, H. E. Reynolds, W. L. Kiser, D. R. Reinecke and G. A. Kruger, "Breast cancer in vivo: Contrast enhancement with thermoacoustic CT at 434 MHz-feasibility study," Radiology 216, 279-283 (2000). 
[18] T. D. Khokhlova, I. M. Pelivanov, V. V. Kozhushko, A. N. Zharinov, V. S. Solomatin and A. A. Karabutov, "Optoacoustic imaging of absorbing objects in a turbid medium: ultimate sensitivity and application to breast cancer diagnostics," Applied Opt. 46, 262-272 (2007).

[19] V. G. Andreev, A. A. Karabutov and A. A. Oraevsky, "Detection of ultrawideband ultrasound pulses in optoacoustic tomography," IEEE Trans. Ultrason. Ferr. Freq. Contr. 50, 1383-1390 (2003).

[20] S. Manohar, A. Kharine, J. C. G. van Hespen, W. Steenbergen and T. G. van Leeuwen, "The Twente photoacoustic mammoscope: system overview and performance," Phys. Med. Biol. 50, 2543-2557 (2005). (doi:10.1088/0031$9155 / 50 / 11 / 007)$

[21] H. Lamela, D. Gallego, R. Gutierrez and A. Oraevsky, "Interferometric fiber optic sensors for biomedical applications of optoacoustic imaging," J. Biophotonics 4, 184-192 (2011). (doi:10.1088/0031-9155/50/11/007)

[22] V. G. Andreev, A. A. Karabutov, S. V. Solomatin, E. V. Savateeva, V. Aleinikov, Y. V. Zhulina, R. D. Fleming and A. A. Oraevsky, "Optoacoustic tomography of breast cancer with arc-array transducer," Proc. SPIE $3916 \mathbf{3 6}$, (2000).(doi:http://dx.doi.org/10.1117/12.386339)

[23] J. W. Hunt, M. Arditi and F. S. Foster, "Ultrasound transducers for pulse-echo medical imaging," IEEE Trans. Ultrason. Ferr. Freq. Contr. BME-30, (1983).

[24] S. Manohar, A. Kharine, J. C. G. van Hespen, W. Steenbergen, and T. G. van Leeuwen, "Photoacoustic mammography laboratory prototype: Imaging of breast tissue phantoms," J. Biomed. Opt. 9, 1172-1181 (2004).

[25] S. Manohar, S. E. Vaartjes, J. C. G. van Hespen, J. M. Klaase, F. M. van den Engh, W. Steenbergen, and T. G. van Leeuwen, "Initial results of in vivo noninvasive cancer imaging in the human breast using near-infrared photoacoustics," Opt. Express 15, 12277-12285 (2007).

[26] M. H. Xu and L. H. V. Wang, "Analytic explanation of spatial resolution related to bandwidth and detector aperture size in thermoacoustic or photoacoustic reconstruction," Phys. Rev. E 67, 056605 (2003). (doi: 10.1103/PhysRevE.67.056605) 
[27] C. G. A. Hoelen, F. F. M. de Mul, R. Pongers and A. Dekker, "Three-dimensional photoacoustic imaging of blood vessels in tissue," Opt. Letter 23, 648-650 (1998). (doi: http://dx.doi.org/10.1364/OL.23.000648)

[28] B. Woodward, S. K. Hole and W. Forsythe, "Transducer design for a correlation log," Ultrasonics 31, 21-33 (1992). (doi: http://dx.doi.org/10.1016/0041$624 \mathrm{X}(93) 90029-Y)$

[29] C. Li, G. Ku and L. V. Wang, "Negative lens concept for photoacoustic tomography," Phys. Rev. E 78021901 (2008). (doi: 10.1103/PhysRevE.78.021901)

[30] M. Pramanik, G. Ku and L. V. Wang, "Tangential resolution improvement in thermoacoustic and photoacoustic tomography using a nagative acoustic lens," J. Biomed. Opt. 14, 024028 (2009). (doi: http://dx.doi.org/10.1117/1.3103778)

[31] W. Xia, D. Piras, M. Heijblom, J. C. G. v. Hespen, S. v. Veldhoven, C. Prins, W. Steenbergen, T. G. v. Leeuwen, and S. Manohar, "Enlarged acceptance angle of a finite-size detector in photoacoustic imaging using acoustic lenses," in Novel Biophotonic Techniques and Applications, H. Sterenborg and I. Vitkin, eds., 8090 of Proceedings of SPIE-OSA Biomedical Optics, no. 80900L, (2011).

[32] J. Folkman, "Tumor angiogenesis," in Cancer Medicine, J. F. Holland, Ed., 5th ed. Hamilton, ON: B. C. Decker, 2000, ch. 9, pp. 132 Ü152.

[33] G. Bergers and L. E. Benjamin, "Tumorigenesis and the angiogenic switch," Nat. Rev. Cancer. 3, 401-410 (2003). (doi: 10.1038/nrc1093)

[34] P. L. M. J. van Neer, G. Matte, M. G. Danilouchkine, C. Prins, F. van den Adel and N. de Jong, "Super-harmonic imaging: development of an interleaved phase-array transducer," IEEE Trans. Ultrason. Ferr. Freq. Contr. 57, 455-468 (2010). (doi: 10.1109/TUFFC.2010.1426)

[35] G. Kossoff, "The effects of backing and matching on the performance of piezoelectric ceramic transducers," IEEE Trans. Sonics Ultrason. SU-13, 20-30 (1966).

[36] B.T. Cox and P.C. Beard, "Fast calculation of pulsed photoacoustic field in fluids using k-space metholds," J. Acoust. Soc. Am. 117(6), 3616-3627 (2005).

[37] R.A. Kruger, D.R Reinecke, G.A. Kruger "Thermoacoustic computed tomography-technical considerations," Med Phys, 26(9), 1832-1837 (1999). 
[38] R. E. McKeighen, "Design guidelines for medical ultrasound arrays," Proc. SPIE Int. Symp. Med. Imag., 3341, 2-4 (1998). (doi: http://dx.doi.org/10.1117/12.307992)

[39] P. Reynolds, J. Hyslop and G. Hayward, "Analysis of spurious resonances in single and multi-element piezocomposite ultrasonic transducers," Proc. SPIE Int. Ultrasonics Symp., 1650-1653 (2003).

[40] S. Sherrit, H. Wiederick, B. K. Mukherjee, and M. Sayer, "Field dependence of the complex piezoelectric, dielectric, and elastic constants of Motorola PZT 3203 HD ceramic," in Smart Struct. Mater. 1997: Smart Mater. Technol., Proc. SPIE, 3040, 99 Ü109 (1997).

[41] P. L. M. J. van Neer, "Ultrasonic superharmonic imaging," Ph.D thesis, Chap. 5, pp. 53-54, (2010).

[42] D. A. Leedom, R. Krimholtz, and G. L. Matthaei, "Equivalent circuits for transducers having arbitrary even- or odd-symmetry piezoelectric excitation," IEEE Trans. Sonics Ultrason. SU-18, 128-141 (1971).

[43] W. Qi, and W. Cao, "Finite element study on 1-D array transducer design," IEEE Trans. Ultrason. Ferr. Freq. Contr. 47, 949-955 (2000).

[44] J. Callerama, R. H. Tancrell and D. T. Wilson, "Transmitters and receivers for medical ultrasonics" Ultrasonic Symposium Proceedings, IEEE CH14829/79/0000-0407 pp. 407-411, (1979).

[45] E. Carstensen, "Self-reciprocity calibration of electroacoustic transducers" J. Acous. Soc. Am., Vol. 19, No. 6, pp. 961-965, (1947).

[46] J. Yin, M. Lee, J. Brown, E. Chérin and F. S. Foster, "Effect of triangular pillar geometry on high-frequency piezocomposite transducers," IEEE Trans. Ultrason. Ferr. Freq. Contr. 57, 957-968 (2010).

[47] G. L. Matthaei, L. Young and E. M. T. Jones, "Mircrowave filters, impedancematching networks, and coupling structures", Artech House, Dedham, Mass. pp. 255-354 (1980).

[48] K. Fukutani, Y. Someda, M. Taku, Y. Asao, S. Kobayashi, T. Yagi, M. Yamakawa, T. Shiina, T. Sugie and M. Toi, "Characterization of photoacoustic 
tomography system with dual illumination", Proc. of SPIE vol. 7899, 78992J (2011).

[49] Y. Wang, D. Xing, Y. Zeng and Q. Chen, "Photoacoustic imaging with deconvolution algorithm," Phys. Med. Biol. 49, 3117-3124 (2004).

[50] K. Wang, R. Su, A. A. Oraevsky, and M. A. Anastasio, "Investigation of iterative image reconstruction in three-dimensional optoacoustic tomography," Phys. Med. Biol. 57, 5399-5422 (2012).

[51] A. Rosenthal, V. Ntziachristos, and D. Razansky, "Model-based optoacoustic inversion with arbitrary-shape detectors," Phys. Med. Biol. 38(7), 4285-4295 (2011).

[52] J. Jose, R. G. H. Willemink, W. Steenbergen, T. G. van Leeuwen and S. Manohar, "Speed-of-sound compensated photoacoustic tomography for accurate imaging," Med. Phys. 39, 7262-7271 (2012). 


\title{
Chapter 4
}

\section{A new acoustic lens material for large area detectors in photoacoustic breast tomography}

\author{
${ }_{4}^{4}$ Abstract
}

A large surface area detector due to its high sensitivity is preferable to detect weak signals in photoacoustic mammography. The lateral resolution is then limited by the narrow acceptance angle of such detectors. Acoustic lenses made of acrylic plastic (PMMA) have been used to enlarge the acceptance angle of such detectors and improve lateral resolution. However, such PMMA lenses introduce image artifacts due to internal reflections of ultrasound within the lenses, the result of acoustic impedance mismatch with the coupling medium or tissue. A new lens is proposed based on the 2-component resin Stycast 1090SI. We characterized the acoustic properties of the proposed lens material in comparison with commonly used PMMA, inspecting the speed of sound, acoustic attenuation and density. We fabricated acoustic lenses based on the new material and PMMA, and studied the effect of the acoustic lenses on detector performance comparing finite element (FEM) simulations and measurements of directional sensitivity, pulse-echo response and frequency response. We further investigated the effect of using the acoustic lenses on the image quality of a photoacoustic breast tomography system using k-Wave simulations and experiments. Our acoustic

\footnotetext{
${ }^{4}$ This chapter has been published as: W. Xia, D. Piras, J. Van Hespen, W. Steenbergen and S. Manohar, "A new acoustic lens material for large area detectors in photoacoustic breast tomography," Photoacoustics 1(2), 9-18, (2013). Reproduced with permission.
} 
characterization shows that Stycast 1090SI has tissue-like acoustic impedance, high speed of sound and low acoustic attenuation. These acoustic properties ensure an excellent acoustic lens material to minimize the acoustic insertion loss. Both acoustic lenses show significant enlargement of detector acceptance angle and lateral resolution improvement from modeling and experiments. However, the image artifacts induced by the presence of an acoustic lens are reduced using the proposed lens compared to PMMA lens, due to the minimization of internal reflections. The proposed Stycast 1090SI acoustic lens improves the lateral resolution of photoacoustic tomography systems while not suffering from internal reflection-induced image artifacts compared a lens made of PMMA.

\subsection{Introduction}

X-ray mammography and ultrasonography, the current routinely used breast imaging methods, have limitations. X-ray mammography besides possessing ionizing hazards, is not reliable for detecting cancer in dense breasts, while ultrasonography suffers from poor soft tissue contrast [1]. Thus there is a need for a breast imaging method, sensitive and specific enough to detect early stages of cancer while using non-ionizing radiation. Optical mammography has the advantage of high optical contrast but suffers from poor spatial resolution caused by multiple light scattering in tissue [2]. Photoacoustic imaging combines light and sound, where a short laser pulse excites ultrasound by thermo-elastic expansion at locations where light is absorbed. The method has the advantages of both high optical contrast and the high resolution possible with acoustic detection [3-10], and shows promise as an alternative imaging method to detect angiogenic markers of breast cancer based on hemoglobin absorption $[11-20]$.

In photoacoustic breast imaging there is a requirement to detect extremely weak signals generated upto several centimeter deep in tissue. A large-area detector is preferable due to its higher sensitivity than a small-area detector. On the other hand, a large-area detector has a small angle of acceptance at the designed frequency [21], while in a tomographic geometry it is required that each US detector's acceptance angle is wide enough to detect signals generated throughout the object for each angular position around the object. The consequence of a narrow acceptance angle is that the lateral resolution of the system is reduced when using a large-area detector [22].

To use a sensitive large-area detector without compromising its acceptance angle, Li et al [23] and Pramanik et al [24] introduced the negative acoustic lens con- 
cept. A hemicylindrical-shape negative lens made from acrylic plastic (PMMA) was epoxied to the surface of a large surface-area ultrasound transducer to enlarge the transducer acceptance angle. The lateral resolution of the PAT system was dramatically improved as expected. However, the authors pointed out that image artifacts could result possibly introduced by ultrasound internal reflections within the PMMA lens [24].

Materials used as acoustic lenses should provide sufficient refraction to provide lensing effect but with minimum insertion losses. Lensing increases with increase of speed of sound difference between the coupling medium and the lens material, while for low acoustic losses, the material should possess low acoustic reflection and attenuation. Therefore, the ideal acoustic lens material must have a speed of sound (SOS) higher than tissue, an acoustic impedance $Z$ as close to that of tissue as possible, and minimal acoustic attenuation (AA). Acrylic plastic (PMMA) due to its high SOS, and low AA remains a choice for acoustic lens material, but acoustic reflection, due to its $Z$ mismatch with soft tissue, causes loss of acoustic signal. Moreover, internal reflections at the boundaries of the lens with tissue causes image degradation [25].

In this work, we introduce an acoustic lens material with excellent properties as above, based on Stycast 1090SI. We compare this material with PMMA, in terms of SOS, AA and $Z$. The angular sensitivity, pulse-echo and frequency response of a large surface area ultrasound detector with and without acoustic lenses are measured and simulated for the two materials. Finally, we compare the performances of a photoacoustic tomographic system when the two lenses are used affixed on a custom-made single-element detector. Imaging quality improvement is achieved for the Stycast 1090SI lens compared to PMMA lens. We conclude that Stycast 1090SI is an excellent candidate for an acoustic lens used in photoacoustic tomography to enlarge the acceptance angle of a large surface area detector.

\subsection{Materials and methods}

\subsubsection{Lens materials}

\subsubsection{Stycast 1090SI}

The resin Stycast 1090SI and the hardener Catalyst 24LV (Henkel, Düsseldorf, Germany) are accurately weighed into a clean container in the ratio of 100:23 in weight. The components are blended by hand for 3-5 minutes, and the bottom and side of the 


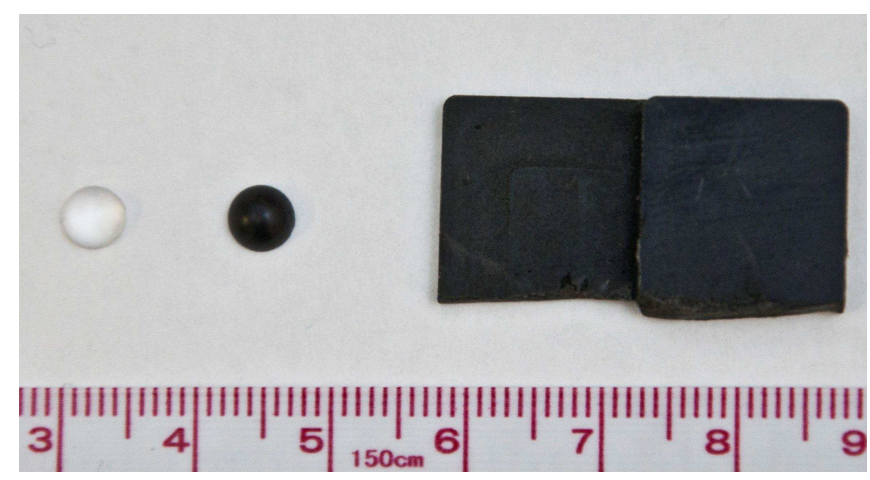

Figure 4.1: Photograph of prepared PMMA and Stycast 1090SI samples: a $4.8 \mathrm{~mm}$ diameter hemispherical PMMA lens (left), a $5 \mathrm{~mm}$ diameter hemispherical Stycast 1090SI lens (middle), and two Stycast 1090SI blocks with dimensions of $15 \times 15 \times 2 \mathrm{~mm}$ and $15 \times 15 \times 4 \mathrm{~mm}$ (right) for acoustic properties characterization.

mixing container are scraped frequently until no sphere-like clusters can be felt. To remove any entrapped air introduced during mixing, the mixture is degassed under vacuum (1-3 mbar) for about 10-15 minutes. During this operation, the foam rises several times the liquid height. We slightly increase the pressure inside the vacuum to let the foam subside. The foam rising and subsiding procedure is repeated to several times. A silicone based mould release (122S, Emerson \& Cuming, Westerlo, Belgium) is applied to the mould using a soft brush, and the mixture is poured carefully into the mould. Before removing the material out of the mould, it is cast for $24 \mathrm{~h}$ at room temperature.

Two Stycast 1090SI blocks with different thicknesses (dimensions of $15 \times 15 \times$ $2 \mathrm{~mm}$ and $15 \times 15 \times 4 \mathrm{~mm}$ ) and hemispherical acoustic lenses with diameter of 5 $\mathrm{mm}$ (Figure 4.1) were prepared. The diameter of the lens is chosen based on the dimensions of the detector surface area $(5 \times 5 \mathrm{~mm})$. The two blocks are used for acoustic characterization and the acoustic lenses are used for studying the transducer angle of acceptance and phantom experiments for photoacoustic tomography.

\subsubsection{Acrylic plastic (PMMA)}

For comparison with Stycast 1090SI lens, PMMA hemispherical lenses are used. These are fabricated by carefully sectioning PMMA balls $(4.8 \mathrm{~mm}$ diameter, Engineering Laboratories, Inc., Oakland, New Jersey) to get hemispheres . Similarly as for Stycast 1090SI, two PMMA blocks (dimensions of $35 \times 35 \times 2.75 \mathrm{~mm}$ and $35 \times 35 \times 3.50 \mathrm{~mm}$ ) are custom-made to assess the acoustic properties. 


\subsubsection{The detector}

A custom-made single-element ultrasound detector was fabricated using CTS 3203HD (CTS Communications Components, Inc., Albuquerque, NM) as the piezoelectric material. The detector with dimensions of active surface $5 \mathrm{~mm}$ x $5 \mathrm{~mm}$ is optimized to have a high sensitivity (0.5 Pa minimum detectable pressure at $1 \mathrm{MHz}$ ) for photoacoustic breast tomography [26]. It has a center frequency of $1 \mathrm{MHz}$ with a $80 \%$ fractional bandwidth. The detector is subdiced to reduce the radial resonance caused by the large thickness/width ratio of the active material CTS 3203-HD. With subdicing, the detector is divided into 25 small units with dimensions of $0.9 \mathrm{~mm} \times 0.9 \mathrm{~mm}$. The units are grouped electrically but separated ultrasonically by air kerfs. More technical and performance details may be found in Ref. [26].

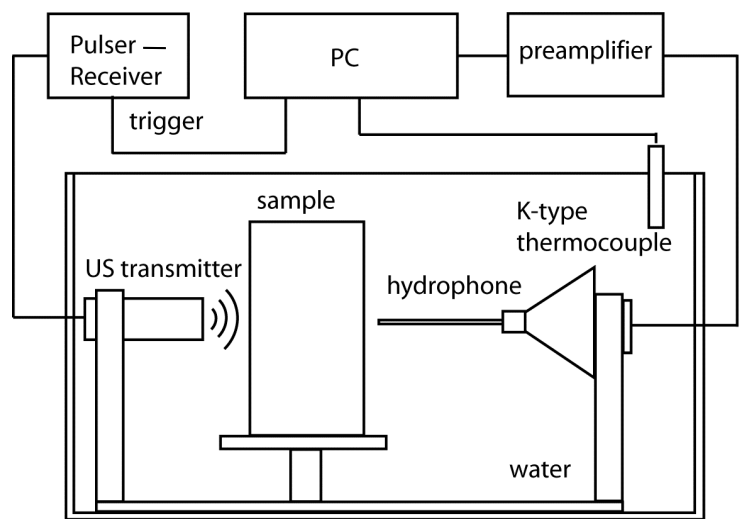

Figure 4.2: Schematic of the setup for the acoustic transmission properties measurements.

\subsubsection{Material acoustic properties characterization methods}

\subsubsection{Speed of sound and acoustic attenuation}

A modified insertion technique is used to measure the acoustic transmission properties (longitudinal speed of sound and acoustic attenuation) [27]. The system configuration is described in detail elsewhere [28]; briefly a single element unfocused Panametrics transducer (for details of central frequencies, see further) together with a broadband needle hydrophone (BLLMCX074 Precision Acoustics Ltd. Dorchester) are mounted in a demineralized water bath at room temperature (Figure 4.2). The transducer is driven by a broadband ultrasonic pulser/receiver (Panametrics 5077PR). The hy- 
drophone measurement data and the water temperature are recorded simultaneously.

For each material under examination, two samples with different known thicknesses (Figure 4.1) are studied. Signals show different time-of-flights $(\Delta T)$ in time domain, and amplitude difference $(\Delta \mathrm{A}(\omega))$ in frequency domain due to the difference in sample thickness $(\Delta d)$. Since the two samples are made of the same material, the acoustic reflections at the water/material interfaces for both samples are equal and canceled during the AA calculation. In this way, inaccuracy in AA estimation caused by acoustic reflection from the sample is minimized.

Knowing the speed of sound of water $\left(C_{\mathrm{w}}\right)$ at the recorded measurement temperature [29], the speed of sound $C_{s}$ and the acoustic attenuation $\alpha_{s}(\omega)\left(\mathrm{dB} \mathrm{cm}^{-1}\right)$ for the unknown material can be calculated in time domain and frequency domain respectively by [31]:

$$
\begin{gathered}
\Delta T=\frac{\Delta d}{C_{\mathrm{w}}}-\frac{\Delta d}{C_{s}} \\
\alpha_{\mathrm{s}}(\omega)=20 \log \left[\frac{\Delta A(\omega)}{\Delta d}\right]+\alpha_{\mathrm{w}}(\omega)
\end{gathered}
$$

where $\alpha_{\mathrm{w}}(\omega)$ the AA of water is negligible since it is small compared to the attenuation of the material [33].

For measurement over a wide frequency range, a set of Panametrics transducers with different center frequencies are used: 1, 2.25, 5, 7.5, and $10 \mathrm{MHz}$ covering the range between $0.4-12 \mathrm{MHz}$. AA data are then taken from a frequency range where the calculated FFT of the measured signal has sufficient signal to noise ratio (SNR) for each transducer. The SOS and AA are measured 5 times for the proposed acoustic lens material Stycast 1090SI and for PMMA.

\subsubsection{Density and acoustic impedance}

The density $(\rho)$ of the proposed lens and of acrylic plastic (PMMA) are measured using a standard pycnometer with deionized water at room temperature as reference. The accuracy of the analytical balance (Sartorius BP 210D, Goettingen) used for measuring the weight is $0.0001 \mathrm{~g}$. Five measurements are made for each material. The acoustic impedance $(Z)$ is then calculated by [31]:

$$
Z=\rho C_{\mathrm{s}}
$$

where $C_{s}$ is the speed of sound of the material whose values are taken from the measurement results in section 2.3.1. 


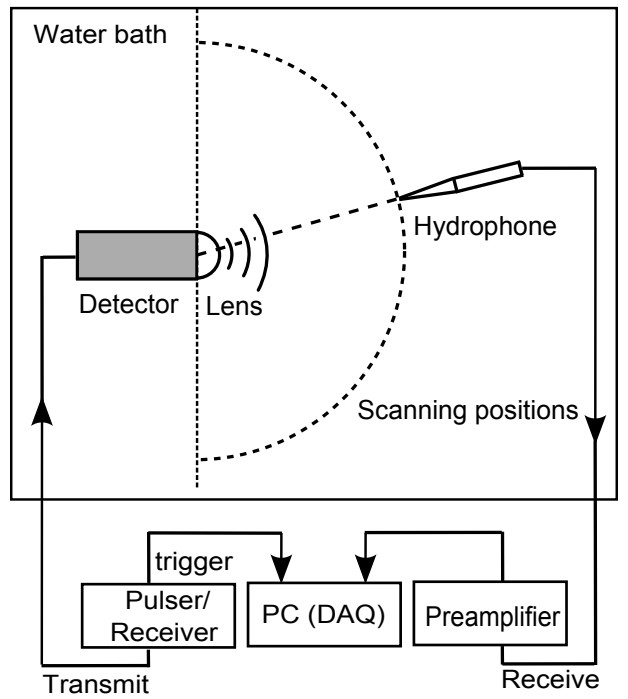

(a)

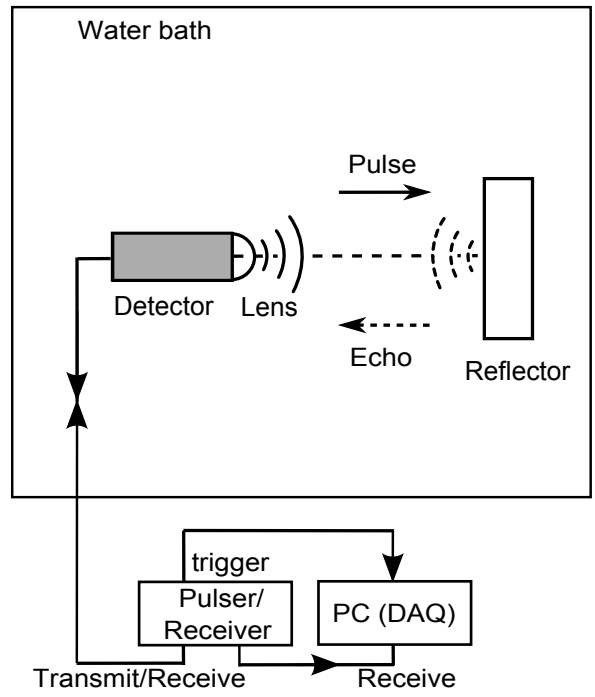

(b)

Figure 4.3: Schematics of (a) directivity measurement setup and (b) pulse-echo signal and electrical-acoustical frequency response measurement setup.

\subsubsection{Detector performance characterization methods}

To study the effect of using acoustic lenses on detector performance, a thin layer of ultrasound gel is sandwiched between the lens and detector. A layer of agar solution is then poured on top of the lens to be gelled for the lens stabilization.

\subsubsection{Directivity (simulation and experiment)}

The acceptance angle of the transducer is simulated and measured in transmission mode, assuming the validity of the reciprocity principle for the piezoelectric material [32]. Simulations are performed using 3D finite-element (FEM) based models using the PZFlex software (version 3.0, Weidlinger Associates Inc, Los Altos, CA). The detailed description of the detector model and material properties of the detector used in simulations are presented in Ref. [26]. The properties of the lens materials used in the simulation are listed in Table 4.1.

For the experimental characterization of the acceptance angle, the detector is mounted in a demineralized water bath at room temperature as transmitter. A broadband needle hydrophone (BLLMCX074 Precision Acoustic Ltd. Dorchester) scans the acoustic field generated by the transducer in the plane covering $180^{\circ}$ in steps of $1^{\circ}$ as shown in Figure 4.3(a). The acoustic signals are recorded with a data acquisition 
Table 4.1: Measured acoustic properties of lens materials (Stycast 1090SI and PMMA) compared with literature values for water. All values are for material at $21{ }^{0} \mathrm{C}$ and for frequency of $1 \mathrm{MHz}$. The longitudinal velocity, shear velocity and acoustic attenuation are used in 3D FEM simulations.

\begin{tabular}{|c|c|c|c|c|}
\hline Acoustic properties & PMMA & Stycast 1090SI & Water & $\mathrm{FML}^{\mathrm{a}}$ \\
\hline Longitudinal velocity $\left(\mathrm{m} \mathrm{s}^{-1}\right)$ & 2757 & 2561 & $1486^{\mathrm{b}}$ & 2068 \\
\hline Shear velocity $\left(\mathrm{m} \mathrm{s}^{-1}\right)$ & $1400^{\mathrm{c}}$ & 900 & - & 994 \\
\hline $\mathrm{AA}\left(\mathrm{dB} \mathrm{cm}^{-1}\right)$ & 1.64 & 1.69 & - & - \\
\hline Density $\left(\mathrm{g} \mathrm{cm}^{-3}\right)$ & 1.20 & 0.56 & 1.00 & 3.14 \\
\hline Impedance (MRayl) & 3.32 & 1.43 & $1.49^{\mathrm{b}}$ & 6.49 \\
\hline
\end{tabular}

a FML: Detector front matching layer, values from Ref. [26].

b Ref. [29].

c Ref. [30]

card (U1067A Acqiris, 8 bit, $500 \mathrm{MS} / \mathrm{s}$ ). Detector acceptance angles without lens, with Stycast 1090SI lens and PMMA lens are measured.

\subsubsection{Frequency response (simulation and experiment)}

The frequency response of the detector, with and without acoustic lenses, is simulated and measured in pulse-echo mode (Figure 4.3(b)). Simulations are performed using 3D FEM models.

For experiments, the detector is driven by a broadband ultrasonic pulser/receiver (Panametrics 5077PR). A stainless steel plate is placed in the far-field ( $\sim 55 \mathrm{~mm})$ of the detector as an acoustic reflector. The frequency response of the detector is calculated by the square root of the FFT of the measured pulse-echo signal to account for the two-way response.

\subsubsection{Acoustic lenses used in photoacoustic tomography ex- periments}

\subsubsection{Forward problem (simulation and experiment)}

The same phantom and tomographic design are used for both simulation and experiment. The phantom has a cylindrical shape $(14 \mathrm{~cm}$ in diameter, with optical absorption $\mu_{\mathrm{a}}=0.003 \mathrm{~mm}^{-1}$, reduced optical scattering $\mu_{\mathrm{s}}^{\prime}=0.4 \mathrm{~mm}^{-1}$ ). Five highly absorbing spherical objects $\left(\mu_{\mathrm{a}}=33 \mathrm{~mm}^{-1}, \mu_{\mathrm{s}}^{\prime}=0.4 \mathrm{~mm}^{-1}\right)$ with diameters of $2 \mathrm{~mm}$ 


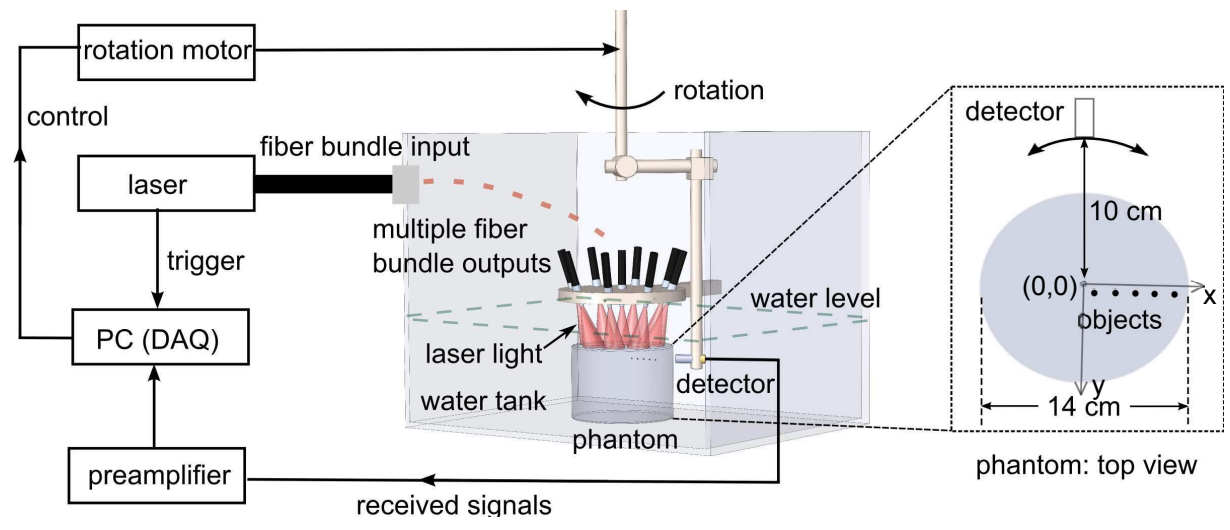

Figure 4.4: Schematic of the measurement configuration for the lenses used in a photoacoustic tomographic system.

are embedded $1 \mathrm{~cm}$ deep in the phantom for 2D imaging as shown in Figure 4.4. The detector scans the phantom over $240^{\circ}$ with scanning step of $2^{\circ}$ with radius of $10 \mathrm{~cm}$. A complete circular scan could not be performed due to mechanical constraints in the form of a holder for the detector. A complete circular scan could not be performed due to mechanical constraints in the form of a holder for the detector. Three experiments are performed using (1) the bare detector, (2) detector with PMMA lens and (3) detector with Stycast 1090SI lens.

The simulations are performed using the k-Wave toolbox [34,35]. A 2D initial pressure distribution map (1024 x 1024 grid, $20 \mathrm{~cm} \mathrm{x} 20 \mathrm{~cm}$ size) is assigned in a 2D tomographic configuration. A initial pressure value of 1 is assigned to the objects, a pressure value of zero is given to background. Measured SOS, $\rho$ and AA of the lens material and medium (water) are assigned (Table 4.1) in the model. A $5 \mathrm{~mm}$ detector is attached to the lens for acoustic wave recording. To simulate the acoustic lens induced internal reflection, SOS and $\rho$ of the detector front matching layer (FML) are assigned to a $5 \mathrm{~mm} \times 10 \mathrm{~mm}$ area with appropriate AA directly behind the $\mathrm{k}$ Wave virtual detector. The time-domain PA signals are averaged over the surface of the detector and then convolved with the measured impulse response of the detector, which is presented in Ref. [26].

For experiments, laser pulses with $10 \mathrm{~Hz}$ repetition rate at $755 \mathrm{~nm}$ wavelength (QCombo, Quanta System, Milan, Italy) are delivered via a fiber bundle (1 input : 9 outputs) to illuminate the phantom from the top (Figure 4.4). The input diameter is $9 \mathrm{~mm}$, and each of the output has a diameter of $3.5 \mathrm{~mm}$ (LGO Optics, San Jose, CA). The phantom and objects are made of agar/Intralipid ( $2 \%$ agar in weight, $0.3 \%$ 
Intralipid in volume $[28,37-40])$, with the object having additonal $5 \%$ India ink for contrast [40]). The phantom is placed in demineralized water in an imaging tank. The laser beam from each fiber output $(\sim 9 \mathrm{~mJ})$ is naturally diverged to cover the entire phantom surface, resulting in less than $1 \mathrm{~mJ} \mathrm{~cm}^{-2}$ fluence at phantom surface. The detector is situated at a distance of $10 \mathrm{~cm}$ from the center of the phantom and rotated to aquire projections mentioned earier. The signals are amplified using a custom-made low noise preamplifier based on ADA4896-2 (Analog Devices, Norwood, MA). The signals are amplified using a custom-made low noise preamplifier based on ADA48962 (Analog Devices, Norwood, MA). The signals are then digitized and transferred (U1067A Acqiris, 8 bit, $500 \mathrm{MS} / \mathrm{s}$ ) to a PC for data processing.

\subsubsection{Image reconstruction}

For both simulations and experiments, k-Wave toolbox is employed for image reconstruction using time-reversal $[35,36]$. A different grid $(1000 \times 1000)$ is used for reconstruction compared to the grid used in the forward simulations (1024 x 1024) to avoid the inverse crime. The SOS of the lens is taken into account by assigning the value to the lens area. To avoid the generation of internal reflections in the lens during the time-reversal reconstruction, the density of each lens is tuned to match the impedance of water and the $5 \mathrm{~mm} \times 10 \mathrm{~mm}$ high impedance region used in the forward simulation is removed.

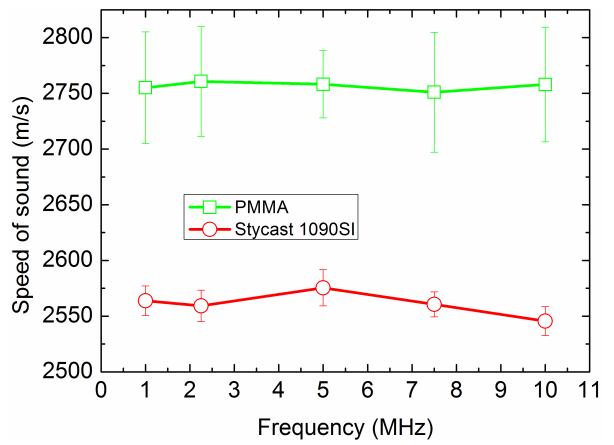

(a)

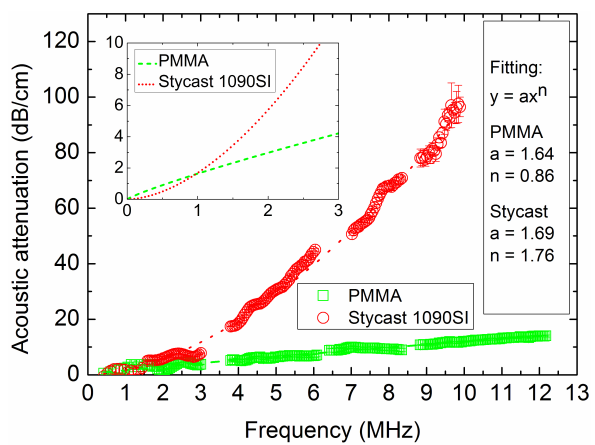

(b)

Figure 4.5: Measured acoustic transmission properties. (a) Speed of sound and (b) acoustic attenuation in Stycast 1090SI and acrylic plastic (PMMA) with frequency power law fitting as insertion for attenuation in low frequency regime. The values are measured at $21{ }^{0} \mathrm{C}$. Each data point represents an average of 5 measurements and error bars represent standard deviations of the measured values. 


\subsubsection{Image contrast analysis}

To estimate the image contrast, for each reconstructed object, a $1 \times 1 \mathrm{~cm}^{2}$ image region of interest (ROI) is considered with object located in its center. The object in the image is defined by including pixels with values upwards of $-6 \mathrm{~dB}$ of the peak intensity, while the area outside this region is defined to be noise. A contrast to noise ratio (CNR) for each object is calculated as the ratio of the root-mean-square (RMS) value of the object and noise pixels.

\subsection{Results}

\subsubsection{Material acoustic properties}

Figure 4.5 shows the measured acoustic transmission properties at different ultrasound frequencies for Stycast 1090SI and acrylic plastic (PMMA) blocks. In general, our measurement results for PMMA agree well with literature values [41,42], indicating the accuracy of our acoustic characterization. The measured SOS (Figure 4.5(a)) is slightly higher for PMMA than for Stycast 1090SI, while both have much larger SOS than water at the measurement temperature of $21^{0} \mathrm{C}$, indicating an efficient enlargement of the transducer acceptance angle when used as acoustic lenses according to Snell's law. For both materials, the SOS is constant for different frequencies, and no dispersion trend can be observed though relatively large standard deviations are observed. Larger standard deviations of the measured $c$ values for PMMA are registered compared to those for Stycast 1090SI. This is because the $\Delta d$ for the two PMMA blocks $(0.75 \mathrm{~mm})$ is smaller than that for Stycast 1090SI (2 mm); the uncertainty in thickness estimation has larger influence on $c$ estimation for PMMA.

The measured AA (Figure 4.5(b)) has low standard deviations and follows the frequency power law as for most materials [25]. The AA for Stycast 1090SI and PMMA can then be expressed from a power law fit to frequency (f, in $\mathrm{MHz}$ ) as $1.64 \mathrm{f}^{0.86}$ and $1.69 \mathrm{f}^{1.76}$ respectively. However for Stycast 1090SI, higher variations are presented at high frequencies due to the large attenuation of the material. Both Stycast 1090SI and PMMA have low AA at low frequency regime (around $1 \mathrm{MHz}$ ), while its value increases with frequency more rapidly for Stycast 1090SI than for PMMA.

The standard deviations for SOS values are relatively large because thin samples are used where there is uncertainty in thickness estimation. The reason for using thin samples is to avoid the heavy attenuation and thus to have high SNR for the 
frequency domain analysis of the detected signals.

The measured $\rho$ of Stycast 1090SI $\left(0.56 \mathrm{gcm}^{-3}\right)$ is lower than that of water, and combined with the high SOS, Stycast 1090SI has Z (1.43 MRayl) close to tissue (water), while PMMA has higher $Z$ (3.32 MRayl) than water (Table 4.1).

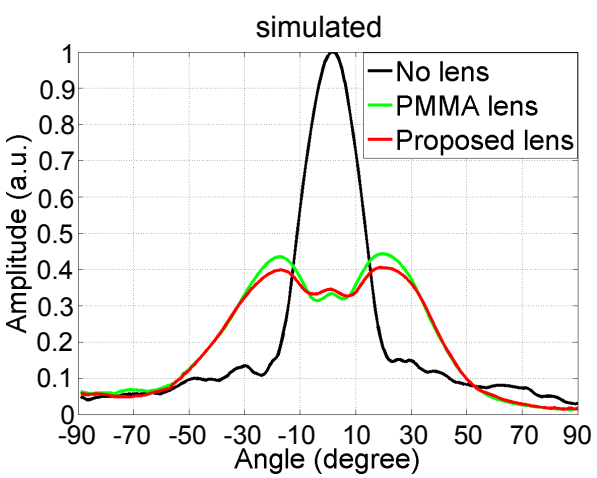

(a)

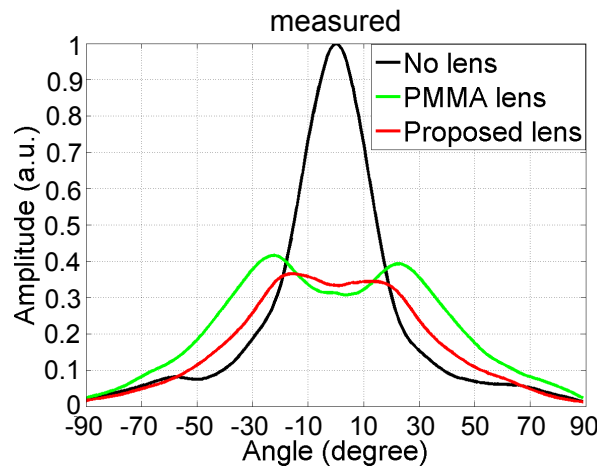

(b)

Figure 4.6: Directional sensitivity of the detector without a lens, with PMMA lens and with proposed lens: (a) simulated and (b) measured.

\subsubsection{Acceptance angle using the acoustic lenses}

The simulated and measured directional sensitivity of the detector with and without lenses are shown in Figure 4.6. In general, the simulation results match the experimental results. The $-6 \mathrm{~dB}$ acceptance angle of the bare detector is $\sim 25^{\circ}$. Both simulated and measured directional sensitivity curves for the detector with lenses have a valley in the center region. The contribution from the uncovered areas enhanced the sensitivity of the detector in front of those areas, forming the valleys in the curves. Since the SOS of PMMA is larger than Stycast, the acceptance angle of the detector with PMMA lens $\left(\sim 90^{\circ}\right)$ is larger than Stycast $\left(\sim 75^{\circ}\right)$.

\subsubsection{Pulse-echo and frequency response}

Pulse-echo and frequency response of the detector with and without lenses are simulated and compared with measurement results in Figure 4.7. In general, the simulation results match the experimental results. The $-6 \mathrm{~dB}$ acceptance angle of the bare detector is $25^{\circ}$ (20 from simulation). Both simulated and measured directional sensitivity curves for the detector with lenses have a valley in the center region. The contribution 

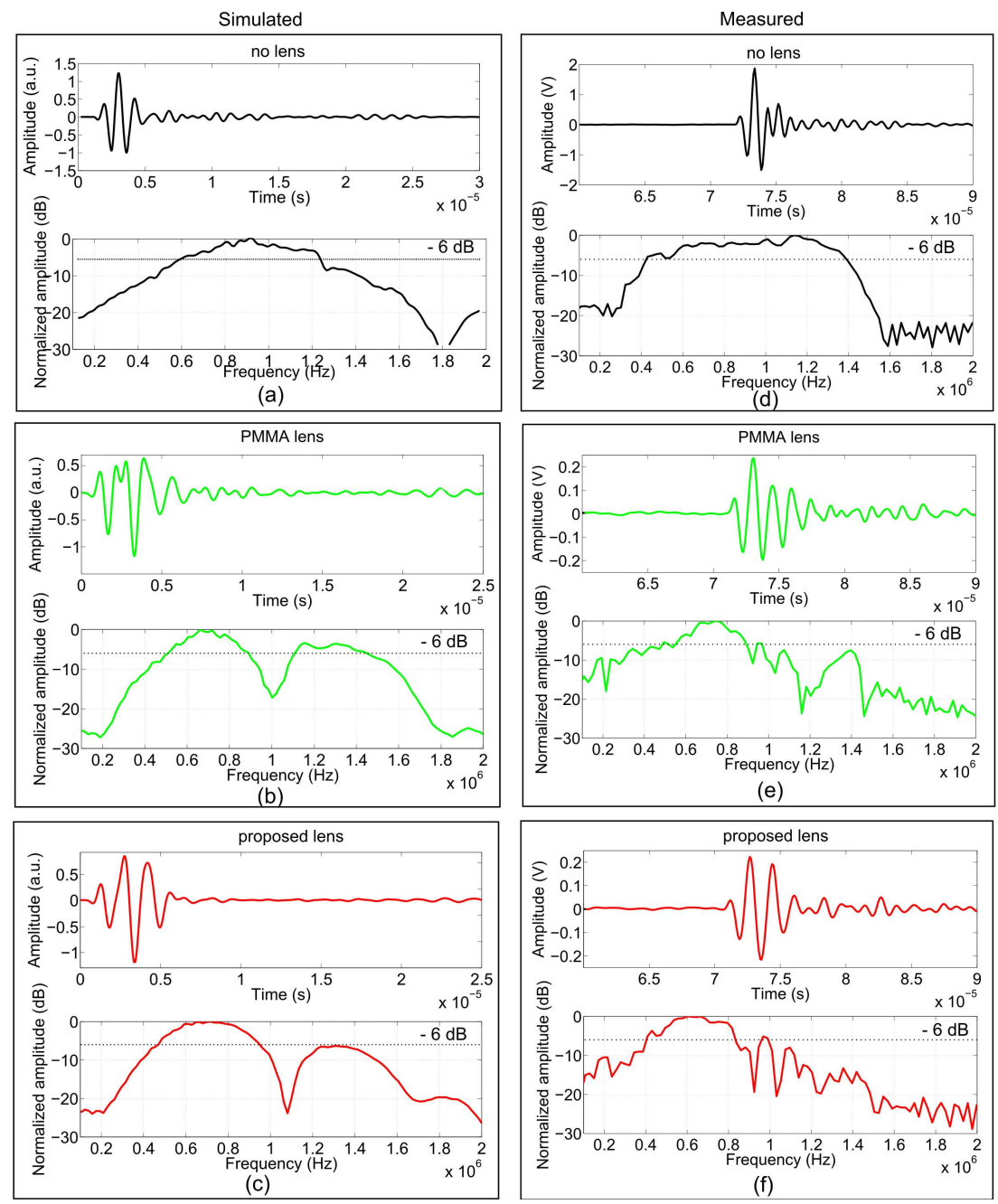

Figure 4.7: Simulated (left column) and measured (right column) pulse-echo and frequency response of the detector: (a) without lens, (b) with PMMA lens, (c) with Stycast 1090SI lens, (d) without lens, (e) with PMMA lens and (f) with Stycast 1090SI lens. (a)-(c) are simulation results signals time-shifted to origin and (d)-(f) are measurement results.

from the uncovered areas enhanced the sensitivity of the detector in front of those areas, forming the valleys in the curves. Since the $c$ of PMMA is larger than Stycast, the acceptance angle of the detector with PMMA lens (95 from measurement, $85^{\circ}$ from simulation) is larger than Stycast $\left(75^{\circ}\right.$ from measurement, $80^{\circ}$ from simulation). 


\subsubsection{Imaging quality}

Figure 4.8 shows the reconstructed images from PAT simulations (left column) and measurements (right column). In general, simulations agree with the measurements. Reconstructions of the objects imaged with the bare detector are shown in Figure 4.8(a)and(i). The objects are less and less visible and elongated in the $\mathrm{Y}$ direction when moving away from the center of rotation at $\mathrm{x}=0, \mathrm{y}=0$. On the other hand, the reconstructions of the objects imaged with both acoustic lenses possess better image quality at all $\mathrm{x}$ : all the objects are clearly visible with well preserved shapes (Figure $4.8(\mathrm{~b}),(\mathrm{c}) \operatorname{and}(\mathrm{j}),(\mathrm{k}))$. The axial and lateral profiles for all objects are shown in Figure 4.8(d),(i), and (e), (m) respectively. The full width at half maximum (FWHM) values of the axial and lateral profiles for each object imaged with and without lenses are shown in Figure 4.8(f)(g)(n) and (o). They indicate that the axial resolution remains the same when the objects are imaged with acoustic lenses attached to the detector as compared to the bare detector, while the lateral resolution improved, with a FWHM reduced from $\sim 4 \mathrm{~mm}$ to $\sim 2 \mathrm{~mm}$ using both PMMA and Stycast 1090SI lenses as expected. We also calculated contrast to noise ratio (CNR) for each object to appreciate the differences in performance between the Stycast 1090SI and PMMA lenses. The CNR was calculated as the ratio of the root-mean-square (RMS) value of the object and noise pixels, with the boundary between the two regions the $-6 \mathrm{~dB}$ points of highest pixel intensity in a ROI around each object. The CNRs of each reconstructed object are shown in Figure 4.8(h) and (p).

Objects imaged with the bare detector have ring-shape artifacts due to the limited bandwidth of the detector (Figure 4.8(a)and(i)). Those ring-shape artifacts are even enhanced for objects imaged by the detector with PMMA lens due to the reverberation of ultrasound inside the lens (indicated by green arrows in Figure 4.8(b)and(j)). This is because the acoustic impedance of PMMA lens lies well between the front matching layer and surrounding water/tissue. On the other hand, objects imaged by Stycast 1090SI lens show much weaker artifacts as compared to PMMA lens. The reduction of image artifacts can be clearly seen from the lateral profiles for the object close to the detection side in Figure 4.8(e)insets. Further the CNRs of objects imaged by the Stycast 1090SI lens are higher than those from the PMMA lens, due to the reflection artifacts associated with the latter (Figure 4.8(h) and (p)). 


\subsection{Discussion}

Li et al [23] introduced the concept of using an acoustic lens to enlarge the narrow acceptance angle of a large surface-area detector, and consequently improve the lateral resolution of the system. Later Pramanik et al [24] applied this concept in their photoacoustic/thermocoustic breast imager using an acoustic lens made of PMMA. Due to the acoustic impedance mismatch between PMMA and detector front matching layer, and between PMMA and the surrounding medium, reverberation of ultrasound inside the lens could cause image artifacts as pointed out by the authors [24].

We introduced a new acoustic lens material Stycast 1090SI, which has a remarkable tissue-like acoustic impedance, which is more suitable than PMMA for use as an acoustic lens in photoacoustic tomography. We detailed the fabrication of the material. Care needs to be taken to prevent air bubbles entrapped inside the material during material manufacturing. We studied the effect of using this acoustic lens on the detector performances and on the image quality of the system. We find that besides improved lateral resolution compared to bare detector, image artifacts are minimized by using the proposed lens compared with PMMA. This is because the material has large speed of sound to enlarge the detector acceptance angle, while having tissue-like acoustic impedance to minimize the ultrasound internal reflections within the lens.

The acoustic attenuation of Stycast 1090SI increases rapidly with frequency (Figure 4.5(b). For frequencies below $1 \mathrm{MHz}$ the acoustic attenuation of the $5 \mathrm{~mm}$ diameter $(2.5 \mathrm{~mm}$ thickness) lens is acceptable making the lens well suited. For frequencies larger than $3 \mathrm{MHz}$, the acoustic attenuation within $5 \mathrm{~mm}$ diameter lens is $\sim 6 \mathrm{~dB}$. Thus, for higher frequency ultrasound detectors it is may be preferable to use PMMA lenses which possess lower insertion losses, which however induce more image artifacts compared to Stycast 1090SI lens.

The center frequency of the detector is lowered due to the acoustic attenuation of both PMMA lens and Stycast lens (Figure 4.7). It is important to take this into account when designing the center frequency of ultrasound detectors. The bandwidth of the detector is reduced by using PMMA due to the internal reflections within the lens, while less bandwidth reduction is found for Stycast lens compared to PMMA lens.

The directivity curves of the detector with both Stycast 1090SI and PMMA lens attached have a valley in the center (Figure 4.6). This is because the detector surface area $(5 \mathrm{~mm} \times 5 \mathrm{~mm})$ is not completely covered by the lens (5 mm diameter). A 
larger lens (7 mm diameter) covering the entire surface area of the detector is found to solve this problem in simulations (not shown in this work). However, using a larger acoustic lens increases the complexity for the detector array development as part of the lens requires to be removed. Since good image quality has been achieved as shown in Figure 4.8, we decided to use an acoustic lens with $5 \mathrm{~mm}$ diameter.

Interestingly the insertion loss for PMMA lens is smaller than for the Stycast 1090SI lens. This is because the acoustic impedance of PMMA lies between water and the front matching layer of the detector, which makes PMMA function as a second impedance matching layer between the detector front matching layer and surrounding water, increasing the detector sensitivity. For Stycast 1090SI lens, due to the large difference between the acoustic impedance of the detector front matching layer and the lens material, the reflection loss is large. To prevent the large reflection loss for Stycast 1090SI lens, the acoustic impedance of the detector front matching layer requires to be tuned lower than the present value. In future, detectors with two or more front matching layer will be designed with acoustic impedance of the outer matching layer close to tissue [44]. In this way, acoustic reflection loss induced by Stycast 1090SI lens will be minimized which will further increase the detector bandwidth.

\subsection{Conclusions}

Stycast 1090SI due to its tissue-like acoustic impedance, low acoustic attenuation and high speed of sound, is an excellent acoustic lens material for photoacoustic imaging at around $1 \mathrm{MHz}$ detection frequencies. Combined with the large surface area ultrasound detector, acoustic lenses made from Stycast 1090SI can enlarge the acceptance angle of the detector, and thus improve the lateral resolution of the photoacoustic tomographic system for breast imaging with minimized image artifacts, compared with earlier used PMMA. 

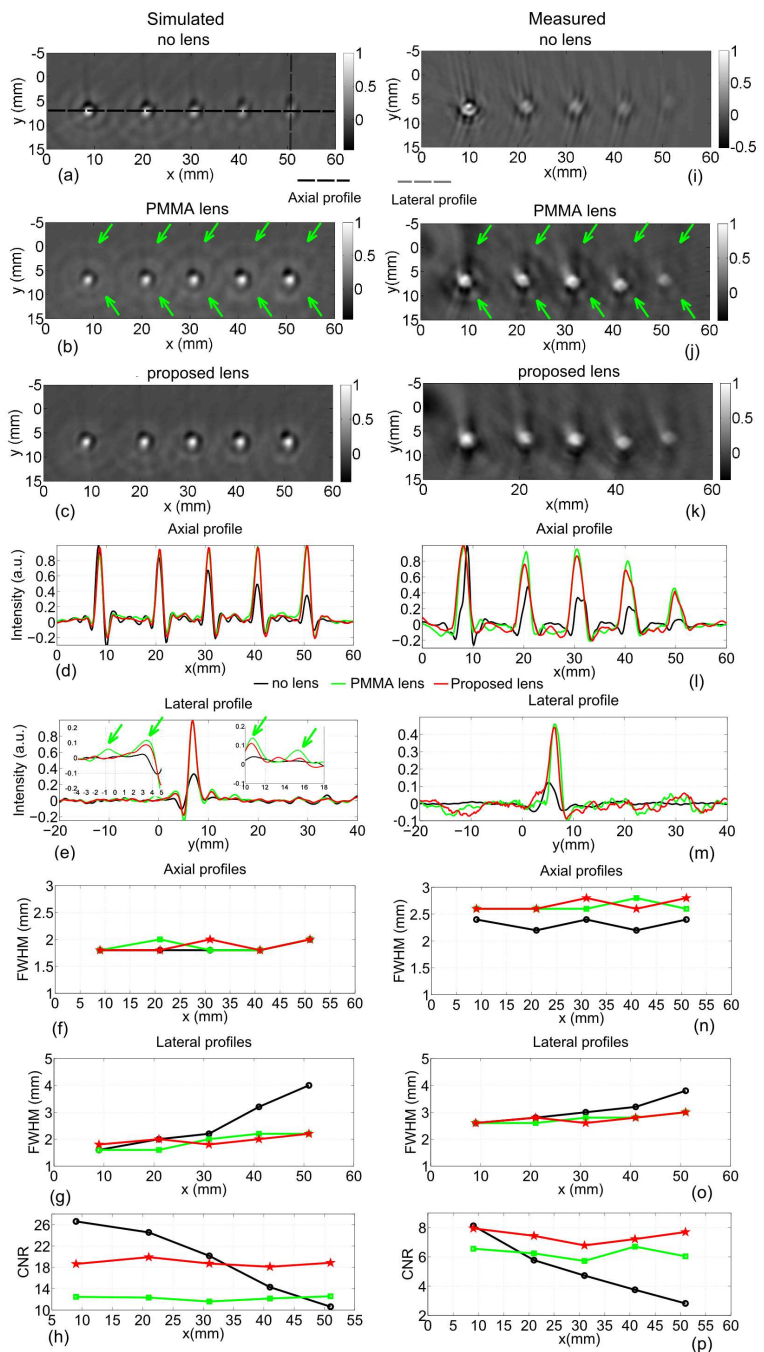

Figure 4.8: Simulated (left column) and experimental (right column) PAT results for the described phantom: (a,i) Objects imaged by detector without an acoustic lens attached, $(b, j)$ by detector with PMMA lens and (c, k) with Stycast 1090SI lens. Each image is normalized to its maximum intensity. $(\mathrm{d}, \mathrm{l})$ Axial intensity profiles along the trajectory indicated by dashed black line from (a)-(c). (e, m) Lateral intensity profiles indicated by dashed gray line from (i)-(k). (a)-(h) are simulation results, (i)(p) are measurement results. Lateral resolution is improved by using both PMMA and Stycast lenses as expected, while axial resolution remains constant for all three cases. Due to limited bandwidth of the detector, ring-shape artifacts are presented around the objects. Those ring-shape artifacts are enhanced for objects imaged by detector with PMMA lens due to the ultrasound trapped inside the lens (indicated by arrows), while for the objects imaged by the detector with Stycast 1090SI lens, much weaker such artifacts are presented due to the tissue-like acoustic impedance of the lens material. 


\section{Bibliography}

[1] C. D. Lehman, C. Isaacs, M. D. Schnall, E. D. Pisano, S. M. Ascher, P. T. Weatherall, D. A. Bluemke, D. J. Bowen, P. K. Marcom, D. K. Armstrong, S. M. Domchek, G. Tomlinson, S. J. Skates and C. Gatsonis, "Cancer yield of mammography, MR, and US in high-risk women: Prospective multi-institution breast cancer screening study," Radiology 244, 381-388, (2007)

[2] B. Tromberg, B. W. Pogue, K. D. Paulsen, A. G. Yodh, D. A. Boas and A. E. Cerussi, "Assessing the future of diffuse optical imaging technologies for breast cancer management," Med. Phys. 35, 2443-2451, (2008)

[3] D. Razansky, M. Distel, C. Vinegoni, R. Ma, N. Perrimon, R. W. Köster and V. Ntziachristos, "Multispectral opto-acoustic tomography of deep-seated fluorescent protein it in vivo," Nat. Photonics 3, 412-417, (2009)

[4] J. Jose, S. Manohar, R. G. M. Kolkman, W. Steenbergen and T. G. van Leeuwen, "Imaging of tumor vasculature using Twente photoacoustic systems," J. Biophoton 2, 701-717, (2009)

[5] L. V. Wang, "Multiscale photoacoustic microscopy and computed tomography," Nat. Photonics 3, 503-509, (2009)

[6] D. Razansky, A. Buehler, and V. Ntziachristos, "Volumetric real-time multispectral optoacoustic tomography of biomarkers," Nat. Protocols 6, 1121-1129, (2011) (doi:10.1038/nprot.2011.351)

[7] J. Gamelin, A. Maurudis, A. Aguirre, F. Huang, P. Guo, L. V. Wang, and Q. Zhu, "A real-time photoacoustic tomography system for small animals," Opt. Express, 17(13), 10489-10498, (2009)

[8] X. Wang, Y. Pang, G. Ku, X. Xie, G. Stoica, and L. V. Wang, "Noninvasive laser-induced photoacoustic tomography for structural and functional in vivo imaging of the brain," Nat. Photonics, 21, 803-806, (2003) 
[9] P. Beard, "Biomedical photoacoustic imaging," Interface Focus 1, 602-631, (2011)

[10] L. Xiang, B. Wang, L. Ji, and H. Jiang, "4-D photoacoustic tomography," Scientific reports $\mathbf{3}, 1113$, (2012) (doi:10.1038/srep01113)

[11] D. Piras, W. Xia, W. Steenbergen, T. G. van Leeuwen and S. Manohar, "Photoacoustic imaging of the breast using the Twente Photoacoustic Mammoscope: Present status and future perspectives," IEEE J. Sel. Topic Quantum. Electron. 16, 730-739, (2010)

[12] R. A. Kruger, R. B. Lam, D. R. Reinecke, S. P. D. Rio and R. P. Doyle, "Photoacoustic angiography of the breast," Med. Phys. 37, 6096-6100, (2010)

[13] S. A. Ermilov, T. Khamapirad, A. Conjusteau, M. H. Leonard, R. Lacewell, K. Mehta, T. Miller and A. A. Oraevsky, "Laser optoacoustic imaging system for detection of breast cancer," J. Biomed. Opt. 14(2), 024007, (2009)

[14] M. Pramanik, G. Ku, C. Li and L. V. Wang, "Design and evaluation of a novel breast cancer detection system combining both thermoacoustic (TA) and photoacoustic (PA) tomography," Med. Phys. 35, 2218-2223, (2008)

[15] M. Heijblom, D. Piras, W. Xia, J.C.G. van Hespen, J.M. Klaase, F.M. van den Engh, T.G. van Leeuwen, W. Steenbergen, and S. Manohar, "Visualizing breast cancer using the Twente photoacoustic mammoscope: What do we learn from twelve new patient measurements?," Opt. Express 20, 11582-11597, (2012) (doi: http://dx.doi.org/10.1364/OE.20.011582)

[16] L. Xi, X. Li, L. Yao, S. Grobmyer, and H. Jiang,"Design and evaluation of a hybrid photoacoustic tomography and diffuse optical tomography system for breast cancer detection," Med. Phys., 39, 2584-2594, (2012)

[17] Z. Xie, X. Wang, R. F. Morris, F. R. Padilla, G. L. Lecarpentier, and P. L. Carson, "Photoacoustic imaging for deep targets in the breast using a multichannel 2D array transducer," SPIE Proceedings, 7899, 1172-1181, (2011)

[18] T. D. Khokhlova, I. M. Pelivanov, V. V. Kozhushko, A. N. Zharinov, V. S. Solomatin and A. A. Karabutov, "Optoacoustic imaging of absorbing objects in a turbid medium: ultimate sensitivity and application to breast cancer diagnostics," Appl. Opt., 46, 262-272, (2007) 
[19] Y. Wang, T. N. Erpelding, L. Jankovic, Z. Guo, J. Robert, G. David and L. V. Wang, "In vivo three-dimensional photoacoustic imaging based on a clinical matrix array ultrasound probe," J. Biomed. Opt., 17(6), 061208, (2012)

[20] F. Ye, S. Yang, and D. Xing, "Three-dimensional photoacoustic imaging system in line confocal mode for breast cancer detection," Appl. Phys. Lett., 97, 213702, (2010) (doi:http://dx.doi.org/10.1063/1.3518704)

[21] A. D. Pierce, "Acoustics: An Introduction to its Physical Principles and Applications," Acoustic society of America, New York, (1989)

[22] M. H. Xu and L. V. Wang, "Analytic explanation of spatial resolution related to bandwidth and detector aperture size in thermoacoustic or photoacoustic reconstruction," Phys. Rev. E 67, 056605, (2003)

[23] C. Li, G. Ku and L. V. Wang, "Negative lens concept for photoacoustic tomography," Phys. Rev. E 78, 021901, (2008)

[24] M. Pramanik, G. Ku and L. V. Wang, "Tangential resolution improvement in thermoacoustic and photoacoustic tomography using a nagative acoustic lens," J. Biomed. Opt. 14(2), 024028, (2009)

[25] J. A. Brown, F. S. Foster, A. Needles, E. Cherin and G. R. Lockwood, "Fabrication and performance of a 40-MHz linear array based on a 1-3 composite with geometric elevation focusing," IEEE Trans. Ultrason. Ferro Freq. Control 54, 1888-1894, (2007)

[26] W. Xia, D. Piras, J. C. G. van Hespen, S. van Veldhoven, C. Prins, T. G. van Leeuwen, W. Steenbergen, and S. Manohar, "An optimized ultrasound detector for photoacoustic breast tomography," Med. Phys. 40(3), 032901, (2013)

[27] J. C. Bamber, "Acoustic characteristics of biological media,"Encyclopedia of Acoustics 4 ed M J Crocker (New York: Wiley) (chap 141), 1703-26, (1997)

[28] W. Xia, D. Piras, M. Heijblom, W. Steenbergen, T. G. van Leeuwen and S. Manohar, "Poly(vinyl alcohol) gels as photoacoustic breast phantoms revisited," J. Biomed. Opt. 16(7), 075002, (2011)

[29] J. Lubbers and R. A. Graaff, "Simple and accurate formula for the sound velocity in water," Ultrasound Med. Biol. 24, 1065-1069, (2008) 
[30] H. J. Sutherland, "Acoustical determination of the shear relaxation functions for polymethyl methacrylate and Epon 828-Z," J. Appl. Phys. 48, 3941-3945, (1978)

[31] P. Fish P, "Physics and Instrumentation of Diagnostic Medical Ultrasound," (Chichester: Wiley), (1990)

[32] J. Callerama, R. H. Tancrell and D. T. Wilson, "Transmitters and receivers for medical ultrasonics" Ultrasonic Symposium Proceedings, IEEE CH14829/79/0000-0407, 407-411, (1979)

[33] M. Greenspan, "Acoustic properties of liquids," American Institute of Physics (AIP) Handbook 3rd edition ed D E Grey (New York: McGraw-Hill) p(3)-87, (1972)

[34] B.T. Cox and P.C. Beard, "Fast calculation of pulsed photoacoustic field in fluids using k-space metholds," J. Acoust. Soc. Am. 117(6), 3616-3627, (2005)

[35] B. E. Treeby, and B. Cox, "k-Wave: MATLAB toolbox for the simulation and reconstruction of photoacoustic wave fields," J. Biomed. Opt. 51(2), 021314, (2010)

[36] B. E. Treeby, E. Z. Zhang and B. Cox, "Photoacoustic tomography in absorbing acoustic media using time reversal," Inverse Problems 26, 115003, (2010)

[37] H. J. Van Staveren, C. J. M. Moes, J. van Marie, S. A. Prahl and M. J. C. van Gemert, "Light scattering in Intralipid-10\% in the wavelength range of 400-1100 nm," Appl. Opt. 30, 4507-4514, (1991)

[38] J. A. Curcio and C. C. Petty, "The near infrared absorption spectrum of liquid water", J. Acoust. Soc. Am., 41(5), 302-304, (1951)

[39] G. M. Hale and M. R. Querry, "Optical constants of water in the 200-nm to 200- $\mu \mathrm{m}$ wavelength region", Appl. Opt., 12(3), 555-562, (1973)

[40] F. Martelli and G. Zaccanti "Calibration of scattering and absorption properties of a liquid diffusive medium at NIR wavelengths. CW method," Opt. Express 15(2), 486-500, (2007)

[41] P. E. Bloomfield, W. Lo and P. A. Lewin, "Experimental study of the acoustical properties of polymers utilized to construct PVDF ultrasonic transducers and the acousto-electric properties of PVDF and P(VDF/TrFE) films," IEEE 
transactions on Ultrasonics, Ferroelectrics, and Frequency Control 47(6), 1397$1405,(2000)$

[42] B. T. Carlson, J. van Deventer, A. Scolan and C. Carlander, "Frequency and temperature dependence of acoustic properties of polymers used in pulse-echo systems," Proceedings of IEEE Ultrasonics symposium, Honolulu, HI, USA, 885$888,(2003)$

[43] K. R. Waters and J. G. Miller, "Causality-imposed (Kramers-Krönig) relationships between attenuation and dispersion," IEEE Trans. Ultrason. Ferro Freq. Control 52, 822-834, (2005)

[44] R. E. McKeighen, "Design guidelines for medical ultrasound arrays," Proc. SPIE Int. Symp. Med. Imag., 3341, 2-4,(1998) (doi: http://dx.doi.org/10.1117/12.307992) 


\title{
Chapter 5
}

\section{Design and evaluation of a}

\section{laboratory prototype system for 3D photoacoustic full breast tomography}

\author{
${ }^{5}$ Abstract
}

Photoacoustic imaging has great potential to visualize vascularization-driven optical absorption contrast for breast cancer detection and diagnosis. State-of-the-art photoacoustic breast imaging systems are promising but are limited either by only a 2D imaging capability or by an insufficient imaging field-of-view (FOV). We present a new design of a laboratory prototype system for 3D photoacoustic full breast tomography and evaluate its performance in imaging challenging phantoms. The heart of the system is an ultrasound detector array optimized for high sensitivity specifically developed for breast imaging. Acoustic lenses are used to enlarge the acceptance angle of the large surface area detector elements to ensure a wide system FOV. We characterized the ultrasound detector array performance in terms of frequency response, directional sensitivity, minimum detectable pressure and inter-element electrical and mechanical cross-talk. Further we evaluated the system performance of the laboratory prototype imager using well-defined tissue mimicking phantoms. The system

\footnotetext{
${ }^{5}$ This chapter has been published as: W. Xia, D. Piras, M. K. A. Singh, J. C. G. Van Hespen, T. G. van Leeuwen, W. Steenbergen and S. Manohar, "Design and evaluation of a laboratory prototype system for 3D photoacoustic full breast tomography," Biomed. Opt. Express 4(11) (2013).
} 
possesses a $2 \mathrm{~mm}$ XY plane resolution and a $6 \mathrm{~mm}$ vertical resolution. A vasculature mimicking object was successfully visualized down to a depth of $40 \mathrm{~mm}$ in a breast phantom. Further, tumor mimicking spherical objects with 5 and $10 \mathrm{~mm}$ diameter at $20 \mathrm{~mm}$ and $40 \mathrm{~mm}$ depths are recovered, indicating high system sensitivity. The system has a $170 \times 170 \times 170 \mathrm{~mm}^{3}$ FOV, which is well suited for full breast imaging. Various recommendations are provided for system performance improvement and to guide this laboratory prototype to a clinical version in future.

\subsection{Introduction}

Breast cancer is the leading cause of cancer death in females worldwide [1]. Early detection increases survival rate [2]. X-ray mammography, ultrasonography and magnetic resonance imaging (MRI) are the commonly used imaging modalities for breast cancer detection and diagnosis. All of these techniques have their limitations: X-ray mammography besides possessing ionizing hazards, is less sensitive in women with dense breasts [3]. Ultrasonography in breast imaging suffers from poor soft tissue contrast [4], operator dependence, and lack of standardization. MRI has high sensitivity but suffers from a limited specificity and a relatively high cost and requires the use of a contrast agent $[5,6]$. Since many of these limitations are related to the fundamental nature of the techniques, there is a great need for an alternative breast imaging modality to detect and diagnose early stages of cancer with high sensitivity and specificity.

Owing to the high and spectroscopically rich optical contrast shown by cancer, optical mammography can potentially solve the problems of the conventional imaging modalities, which in addition to the above mentioned drawbacks focus primarily on morphological changes. However, the method suffers from poor resolution due to strong light scattering in biological tissue [7]. Photoacoustic (PA) imaging addresses this limitation by allowing the absorbing chromophores to emit ultrasound (US) waves in response to short pulsed laser light excitation by thermo-elastic expansion. The US waves arrive at the detection site at the tissue surface with less losses and scattering than light in optical mammography. The mathematical reconstruction for PA imaging is much easier than that for optical mammography. Marrying light and sound, PA imaging on the one hand takes the advantages of optical imaging, offering vascularization-driven optical absorption tumor contrast, and on the other, the advantages of ultrasound imaging, offering a superior ultrasound resolution with a large imaging depth [8-15]. The method holds tremendous promise to detect angiogenic 
markers of breast cancer based on hemoglobin absorption [16-26].

Several photoacoustic (thermoacoustic) dedicated breast imaging systems have been reported in the past decade [16-18,21,22,25,26]. The group of Oraevsky developed a semi-circular laser optoacoustic imaging system (LOIS) which has undergone several iterations $[18,19,27]$. The detection is based on an arc-shaped array of $64 / 32$ polyvinylidene fluoride (PVDF) detector elements, with a $1 \mathrm{MHz}$ center frequency and an ultra-broad bandwidth (upto $2.5 \mathrm{MHz}$ ). Two excitation wavelengths (1064 and $757 \mathrm{~nm}$ ) have been employed [18,28]. The latest system promises to faithfully recover deep embedded objects with sub-millimeter resolution [18]. However, the system design gives the detector a slice-shape focusing area, visualizing only a cross-sectional breast slice.

The group of Wang developed a circular breast imaging system combining photoacoustic (PAT) and thermoacoustic tomography (TAT) [22]. TAT is the microwave excited analog of photoacoustics, and potentially provides additional information of increased ionic water concentration associated with the neovascularization. TAT also has potentially a superior imaging depth compared to PAT, which has been previously demonstrated by the group of Kruger [29]. The Wang system excitation uses $0.5 \mu \mathrm{s}, 3.0 \mathrm{GHz}$ microwave and $6.5 \mathrm{~ns}, 1064 \mathrm{~nm}$ wavelength laser pulses. Ultrasound detection is with $13 \mathrm{~mm} / 6 \mathrm{~mm}$-diameter non-focused detectors with $2.25 \mathrm{MHz}$ center frequency (ISS $2.25 \times 0.5 \mathrm{COM}$, Krautkramer). Good quality PAT and TAT images on tissue phantoms have been achieved, however, the present system is still only capable of performing 2D slice imaging.

The group of Jiang recently demonstrated a hybrid PAT and diffuse optical tomography (DOT) system based on a circular detection geometry for breast imaging [25], which combined the high resolution possible with PAT with the spectroscopic functional information possible with DOT [30]. However, the system design only allows for 2D imaging.

These PA systems are promising for breast cancer imaging, but they suffer from poor cross-plane spatial resolution. The group of Kruger recently reported a 3D PAT system based on a hemispherical geometry, which overcomes the limitation of 2D imaging, providing an uniform spatial resolution [17]. The excitation uses $800 \mathrm{~nm}$ wavelength while US detection employs 128 detector elements, $3 \mathrm{~mm}$ in diameter with a $5 \mathrm{MHz}$ center frequency, arrayed in a spiral pattern covering the inner surface of a hemispherical bowl. Breast vasculature down to a depth of $40 \mathrm{~mm}$ was successfully visualized with submillimeter resolution [17]. However, since US detection was initially optimized for small animal imaging [31], the authors recommend the use of a 
lower frequency detector for better sensitivity. Another limitation of the system is the limited field-of-view (FOV) $\left(64 \times 64 \times 50 \mathrm{~mm}^{3}\right)$ for full breast imaging due to the narrow acceptance angle of the detector at high center frequency.

Our group has developed a planar geometry imager, the Twente Photoacoustic Mammoscope (PAM) [32]. The US detection is based on a 2D array of 590 PVDF detector elements, with a 2 x $2 \mathrm{~mm}$ surface area, a $1 \mathrm{MHz}$ center frequency and a 130\% fractional bandwidth. Light excitation uses $1064 \mathrm{~nm}$ wavelength. The system is capable of 3D imaging with 2.3-3.9 mm spatial resolution. We reported our first clinical experiences in 2007, where we showed that carcinoma could be visualized in 4 of the 5 cases studied [33]. Recent clinical results in 2012 corroborated the earlier studies, while showing the successful visualization of malignancies with a superior imaging contrast to X-ray mammography [24]. Further, the contrast of PAM was found to be independent of fibroglandular breast density. However, the system suffers from a small FOV (40 x $\left.40 \times 50 \mathrm{~mm}^{3}\right)$ and a relatively low sensitivity of the PVDF detector.

In this article we describe a new system which addresses many of the limitations of current breast imagers. This laboratory prototype is implemented in a cylindrical geometry using a linear US detector array optimized for ultra-high sensitivity [36], and permits 3D imaging to be performed. We present the system design with special attention to a new detector array and its characterization, such as frequency response, directional sensitivity, minimum detectable pressure (MDP) and inter-element crosstalk. We further evaluate the resulting performance of the imaging system in terms of spatial resolution, sensitivity, imaging quality and FOV using well characterized tissue phantoms. We finally discuss the various recommendations to guide this laboratory prototype system towards a clinical version, which will be the second generation Twente Photoacoustic Mammoscope (PAM-II).

\subsection{Materials and Methods}

\subsubsection{The system}

\subsubsection{Light source and light delivery system}

The schematic of the new laboratory prototype system is shown in Figure 5.1(a). A laser system combining a Q-switched Alexandrite and a Q-switched Nd:YAG is used for NIR light excitation (QCombo, Quanta System, Milan, Italy). The laser emits 60 -ns pulses at $755 \mathrm{~nm}$ and 5-ns pulses at $1064 \mathrm{~nm}$ with a $10 \mathrm{~Hz}$ repetition rate for 


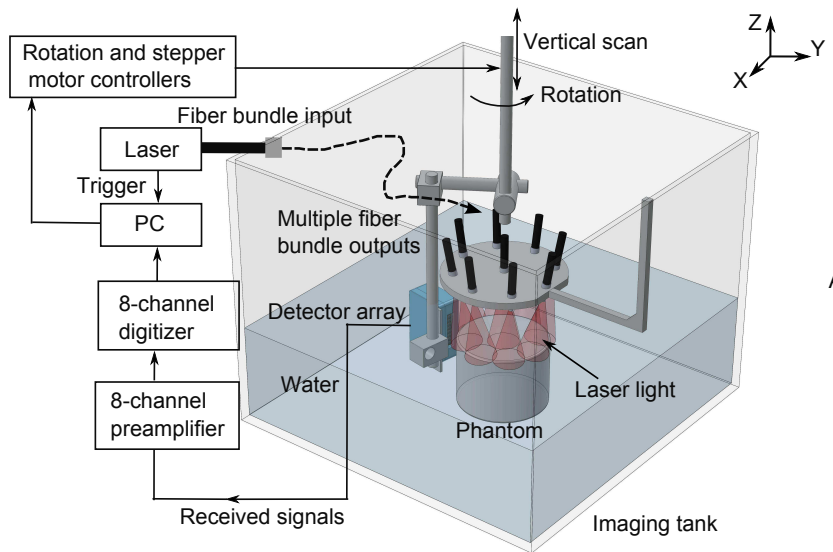

(a)

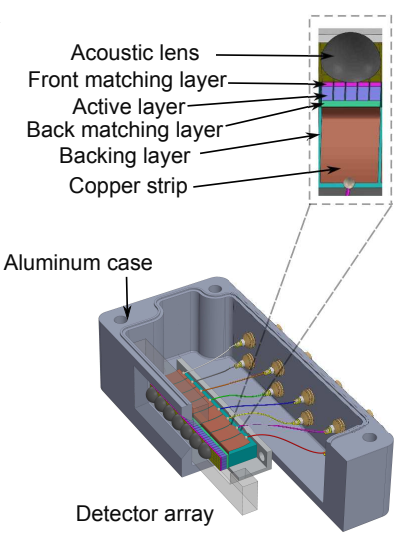

(b)

Figure 5.1: Schematics of (a) the PAM-II laboratory prototype system and (b) the linear array detector.

both wavelengths. The maximum output pulse energy for both wavelengths is around $300 \mathrm{~mJ}$, which can be reduced using motorized attenuators. The laser pulses at both wavelengths share the same optical path reaching the output of the unit. The laser is operated either in a single-wavelength mode (755 nm or $1064 \mathrm{~nm}$ ) with a $10 \mathrm{~Hz}$ repetition rate or in a double-wavelength mode interleaving the two wavelengths with a $20 \mathrm{~Hz}$ repetition rate.

The output laser beam is coupled into a fiber bundle (1 input : 9 outputs) and illuminates the breast phantom as shown in Figure 5.1(a) to simulate bottom illumination of the breast when the patient lies in prone position as in Ref. [17]. The input diameter of the fiber bundle is $9 \mathrm{~mm}$, and each of the output has a diameter of 3.5 mm (LGO Optics, San Jose, CA). The laser beam is naturally spread out from each output to cover the entire breast (or breast phantom) surface. The maximum output laser energy that can be delivered from each fiber output is around $9 \mathrm{~mJ}$. Other light sources were also considered, such as dye lasers and Ti: Sapphire lasers, which have a wider wavelength tuning compared to the dual-head laser used. However, theses lasers were not chosen predominantly due to their insufficient pulse energies.

The $1064 \mathrm{~nm}$ wavelength is selected for the large optical penetration depth in breast tissue [34]. At this wavelength, in addition to absorption by predominantly oxy-hemoglobin, the contributions to PA signals from water, lipid and collagen may be non-negligible. Thus a second wavelength at $755 \mathrm{~nm}$ is chosen where the absorption by water and lipid is low, with deoxy-hemoglobin having a local peak $[18,26,28,35]$. 
However, only $755 \mathrm{~nm}$ wavelength is used in this work for phantom experiments.

\subsubsection{Ultrasound detector array}

An 8-element linear array prototype detector is used for the US detection. The detector array is based on the design of a single-element detector optimized for high sensitivity for PAT of the breast. The detailed description of the design and development of this single-element detector is presented in Ref. [36] and shown in Figure 5.1(b). Briefly each single-element of the detector array is made of highly sensitive active PZT material of $1.625 \mathrm{~mm}$ thickness (CTS 3203HD, CTS Communications Components, Inc., Albuquerque, NM), a front matching layer (0.7 mm thickness, electrical conductive epoxy), a back matching layer (0.48 mm thickness, electrical conductive epoxy) and a backing layer (10 mm thickness) with a $5 \times 5 \mathrm{~mm}$ surface area. Each detection element is sub-diced by cutting $0.1 \mathrm{~mm}$ air kerfs to reduce radial resonances caused by the large thickness/width ratio of the active material. With sub-dicing through the front matching layer and active material, an element is subdivided into 25 small units with dimensions of $0.9 \times 0.9 \mathrm{~mm}$ (Figure 5.1(b)). Thus the sub-units are separated acoustically by the air kerfs while being grouped electrically by an aluminium foil (see further).

For fabricating the detector array (Figure 5.1(b)), a block of the PZT material of dimensions $40 \times 5 \times 1.625 \mathrm{~mm}$ is first diced into individual elements with a 0.15 $\mathrm{mm}$ air kerf cutting through the front matching layer into the backing layer. The center-center distance of each element is $5 \mathrm{~mm}$. A layer of thin aluminum foil is epoxied on top of the front matching layer and is used as the electrical grounding. To protect the aluminum foil, a thin kapton foil layer is glued on top. The signal for each element is taken from a copper strip connected to the electrical conductive back matching layer. A $5 \mathrm{~mm}$ diameter hemispherical acoustic lens made of Stycast 1090SI (Henkel, Düsseldorf, Germany) possessing well-suited acoustic properties is attached on top of each element to enlarge the acceptance angle of the detector for full breast imaging [37]. The detector array is fixed inside an aluminum case with a rectangular aperture with the same dimensions as the detector array. The surface of the detector array through the aperture is flush with the aperture of the aluminum box to have complete electromagnetic shielding.

The detector array is situated at a distance of $11 \mathrm{~cm}$ from the center of the phantom and rotated around it to acquire projections with top-illumination (Figure 5.1(a)). The detector array is scanned along vertical direction to acquire multiple sets of projections to compensate for the large center-center distance between elements 
$(5 \mathrm{~mm})$.

\subsubsection{Data acquisition}

The PA signals detected by the detector array are amplified by an 8-channel prototype low noise preamplifier based on ADA4896-2 (Analog Devices, Norwood, MA) with a $\sim 20 \mathrm{~dB}$ gain as the analog front-end and acquired using a high-speed 8-channel digitizer (NI PXI-5105, 60 MS/s, 12-Bit, National Instruments, Austin, TX) and saved for data processing.

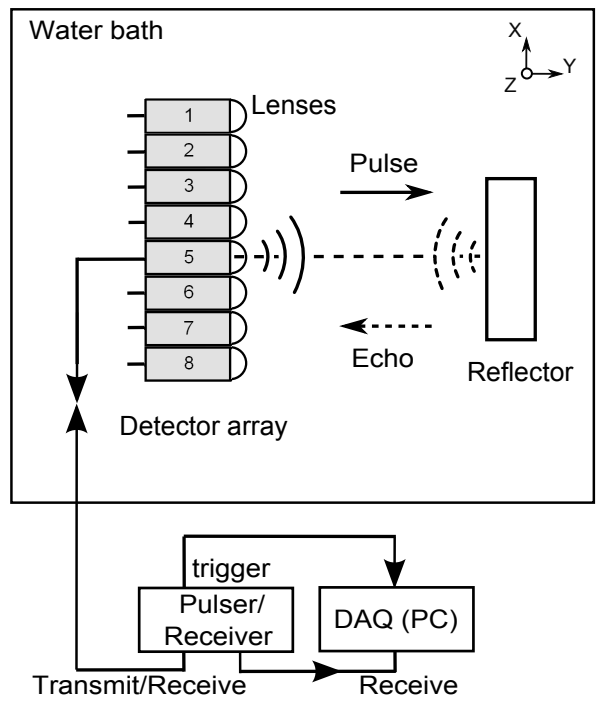

(a)

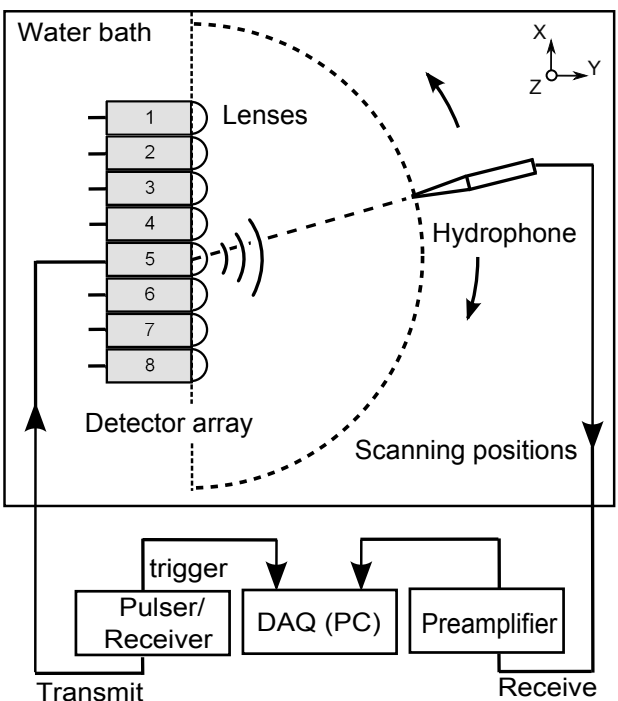

(b)

Figure 5.2: Schematics of measurement setups used for studying (a) pulse-echo and frequency responses and (b) directional sensitivity.

\subsubsection{Detector frequency response}

The frequency response of a typical single detector element (element 5) in the array is measured in pulse-echo mode (Figure 5.2(a)), based on the validity of the reciprocity principle for the piezoelectric material [38]. The detector element is driven by a broadband ultrasonic pulser/receiver (Panametrics 5077PR). The transmitted US pulse is reflected by a stainless steel plate situated in the far-field ( $\sim 55 \mathrm{~mm}$ away) perpendicular to the detector element . The pulse-echo signal is received by the same 
detector element using a data acquisition (DAQ) card (U1067A Acqiris, 8 bit, 500 $\mathrm{MS} / \mathrm{s}$ ). The frequency response of the detector is calculated by the square root of the FFT of the measured pulse-echo signal to account for the two-way response.

\subsubsection{Detector directional sensitivity}

The directivity of the detector element 5 is measured in transmit mode (Figure 5.2(b)). The detector array is mounted in a demineralized water bath at room temperature. The detector element is driven by the broadband ultrasonic pulser/receiver (Panametrics 5077PR) as a transmitter. A calibrated needle hydrophone (BLLMCX074 Precision Acoustics Ltd. Dorchester) is rotated in the far-field centered on the detector element to probe the emitted US field in the plane covering $180^{\circ}$ in steps of $1^{\circ}$. The acoustic signals are acquired using a DAQ card (U1067A Acqiris, 8 bit, $500 \mathrm{MS} / \mathrm{s}$ ). For each scanning position, the peak-to-peak value of the received US pulse is noted and plotted as a function of the scanning angle, giving the directional sensitivity of the detector element at its center frequency.

\subsubsection{Detector sensitivity and minimum detectable pressure}

To ascertain sensitivity and minimum detectable pressure (MDP) of the detector, a substitution method is used where first a $0.5 \mathrm{MHz}$ focused Panametrics broadband transducer is used as a transmitter driven by a 100 cycles sine wave at $0.6 \mathrm{MHz}$ (the central frequency of the detector, see results Figure 5.4(b)) to insonify one detector element in the array in the far-field with progressively reducing pressures till the lowest input possible. Signals from the detector element under investigation after 100 averages and amplification by the 8-channel prototype low noise preamplifier are recorded by the 8-channel digitizer. The root mean square (RMS) value of the signals are calculated and defined as measured voltages. Next the calibrated needle hydrophone is used to measure the pressures from the US transmitter in separate measurements. Finally, the electrical noise (no average) is measured separately when the detector has no driving signal, and its RMS value is calculated. The measured voltages are plotted against pressure inputs and the trend (linear fit) is extended to intersect the voltage noise floor (noise RMS). The slope of the trend gives the sensitivity, and the pressure at the intersection point of the trend with noise level is the MDP (or noise equivalent pressure) at the detector center frequency $(0.6 \mathrm{MHz})$.

The measurement procedure is repeated for each detector element, giving the endof-cable sensitivity and minimum detectable pressure for the US detection system 
chain including the US detector, the preamplifier and the digitizer.

\subsubsection{Detector inter-element electrical and mechanical crosstalk}

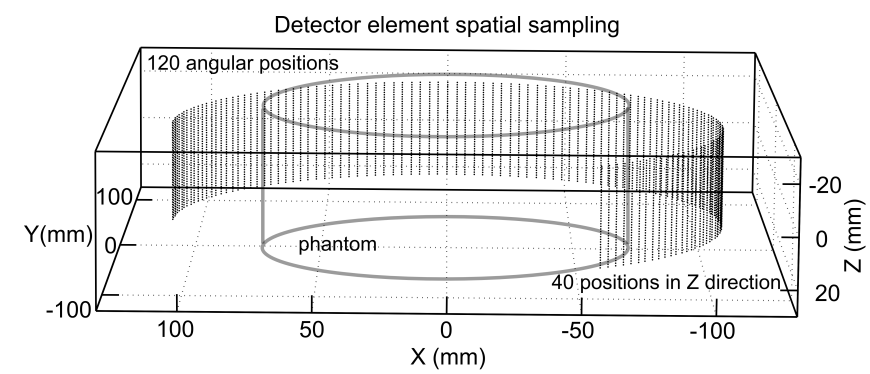

(a)

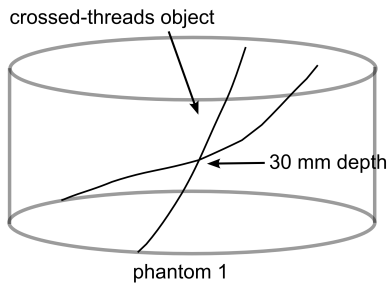

(b)

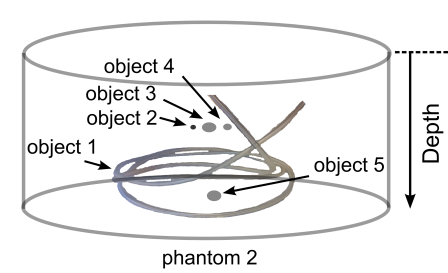

(c)

Figure 5.3: (a) Schematic of the tomographic system showing the relative positions between the scanning detector element and the phantom. (b) Schematic of phantom 1: a crossed-threads object embedded in Agar/Intralipid gel cylinder. (c) Schematic of phantom 2: five objects embedded in Agar/Intralipid gel cylinder. Detailed descriptions of the phantom and object properties are listed in Table 5.1. The lower part of the phantom is not shown in all three schematics.

The levels of electrical and acoustic separation between detector elements are measured in a demineralized water bath by driving element 5 with a 1 cycle sine burst at the detector center frequency $(0.6 \mathrm{MHz})$ with a peak-peak amplitude of 5 $\mathrm{V}[39,40]$. The responses of all elements are directly sent to the 8-channel digitizer. The electrical leakage (electrical crosstalk) from the driven element to the rest of the elements can be recognized by the lack of phase-shift relative to the driven signal. On the other hand, the mechanical crosstalk has a time delay relative to the driving signal depending on the distance from the element of interest to the active element. 
Table 5.1: Background and object properties of phantom 2. Their dimensions, distances from phantom surface (depths), optical reduced scattering coefficient $\mu_{\mathrm{s}}^{\prime}$, and optical absorption coefficient $\mu_{\mathrm{a}}$ are listed. The optical properties of normal and cancerous breast tissue are derived from Ref. [45] at $755 \mathrm{~nm}$ and human blood is derived from Ref. [46] at $800 \mathrm{~nm}$, where blood absorption is independent of the oxygen saturation level.

\begin{tabular}{llllll}
\hline \hline & dimensions & $\begin{array}{l}\text { depth } \\
(\mathrm{mm})\end{array}$ & $\begin{array}{l}\mu_{\mathrm{s}}^{\prime} \\
\left(\mathrm{mm}^{-1}\right)\end{array}$ & $\begin{array}{l}\mu_{\mathrm{a}} \\
\left(\mathrm{mm}^{-1}\right)\end{array}$ & equivalent to \\
\hline background & diameter $13 \mathrm{~cm}$ & - & 0.7 & 0.003 & normal breast tissue $^{\mathrm{a}}$ \\
object 1 & inner diameter $1 \mathrm{~mm}$ & $5-40$ & 0.7 & 0.1 & blood vessel $^{\mathrm{b}}$ \\
object 2 & $2 \mathrm{~mm}$ sphere & 20 & 0.7 & 0.1 & blood vessel $^{\mathrm{b}}$ \\
object 3 & $10 \mathrm{~mm}$ sphere & 20 & 0.7 & 0.01 & tumor $^{\mathrm{a}}$ \\
object 4 & $5 \mathrm{~mm}$ sphere & 20 & 0.7 & 0.01 & tumor $^{\mathrm{a}}$ \\
object 5 & $10 \mathrm{~mm}$ sphere & 40 & 0.7 & 0.01 & tumor $^{\mathrm{a}}$ \\
\hline \hline
\end{tabular}

${ }^{\text {a }}$ Ref. [45].

b Ref. [46].

\subsubsection{Phantom experiments}

\subsubsection{Detector scanning procedure}

The detector array is oriented in vertical direction situated at a distance of $11 \mathrm{~cm}$ from the rotation center to scan the phantom covering $240^{\circ}$ in steps of $2^{\circ}$ to acquire PA signals (100 averages) as shown in Figure 5.3(a). A complete circular scan could not be performed due to mechanical constraints (holder for the detector). When this rotational scan is complete, the detector is moved back to the original position and then moved $1 \mathrm{~mm}$ lower to perform another rotational scan. Five such rotational scans are performed to increase the density of the detector spatial sampling in vertical direction (Figure 5.3(a)). The total number of detection positions can then be calculated as $8 \times 5 \times 120=4800$. A full volume scan in the laboratory prototype takes around 1 hour.

\subsubsection{Phantoms}

Two phantom experiments are performed. Phantom 1 is used to study the system resolution and FOV. Two red nylon fishing threads (0.28 mm diameter) are crossed in the middle and embedded in a Agar/Intralipid gel phantom (2\% agar in weight, Sigma-Aldrich, Germany, and 0.3\% Intralipid in volume, batch no. WI15893, Fresnius 
Kabi Nederland BV, The Netherlands) with an optical reduced scattering coefficient $\mu_{\mathrm{s}}^{\prime}=0.35 \mathrm{~mm}^{-1}[41,42]$, and an optical absorption coefficient $\mu_{\mathrm{a}}=0.003 \mathrm{~mm}^{-1}[43,44]$ at $755 \mathrm{~nm}$ wavelength. The phantom has a cylindrical shape with a diameter of $\sim 130$ $\mathrm{mm}$ and with a height of $\sim 120 \mathrm{~mm}$. The fishing threads are embedded in the upper part of the cylinder and span over $\sim 60 \mathrm{~mm}$ depth as shown in Figure 5.3(b). The phantom is mounted on a heavy metal plate to stabilize the phantom at the bottom of the imaging tank. The PA signals and acoustic reflections from the metal plate are well separated from the PA signals from the object.

Phantom 2 is used to study the sensitivity of the system. The phantom background has the similar design used for phantom 1 only with higher Intralipid concentration ( $0.6 \%$ Intralipid in volume) to mimick $\mu_{\mathrm{s}}^{\prime}$ and $\mu_{\mathrm{a}}$ of normal breast tissue at $755 \mathrm{~nm}$ [45]. Various inhomogeneities are embedded in the phantom. Object 1 is a soft plastic tube (1 mm inner diameter, LsmaTec, Glattbrugg, Switzerland) filled with Agar gel formed in an aqueous dilution of Intralipid and Indian ink (Royal Talens, the Netherlands) for $\mu_{\mathrm{a}}=0.1 \mathrm{~mm}^{-1}$. The tube is arranged in a spiral pattern spanning over $40 \mathrm{~mm}$ depth. Objects 2 to 5 comprise four spherical objects made of the same Agar gel, embedded at different depths inside the phantom (Figure 5.3). These objects have various diameters (Table 5.1), and optical absorptions (object 2 has $\mu_{\mathrm{a}}=0.1 \mathrm{~mm}^{-1}$, objects $3-5$ have $\mu_{\mathrm{a}}=0.01 \mathrm{~mm}^{-1}$ ). The detailed description of the object location, dimensions, and their optical properties are listed in Table 5.1. The spherical objects are made of Agar/Intralipid as for the phantom background, with an additional Indian ink dilution.

The ink dilutions above are obtained from a stock solution of $1 \% \mathrm{v} / \mathrm{v}$ ink in water. The $\mu_{\mathrm{a}}$ of the solution is measured using a spectrophotometer (Shimadzu UV-3101 PC, Tokyo, Japan) possessing a $\mu_{\mathrm{a}}$ of $0.25 \mathrm{~mm}^{-1}$ at $755 \mathrm{~nm}$ and is used as a batch solution. The batch solution is then diluted to give appropriate absorptions for the objects to mimic either tumor tissue $\left(0.04 \% \mathrm{v} / \mathrm{v}\right.$ Indian ink in water, $\left.\mu_{\mathrm{a}}=0.01 \mathrm{~mm}^{-1}\right)$ or human blood aborption $\left(0.4 \% \mathrm{v} / \mathrm{v}\right.$ Indian ink in water, $\left.\mu_{\mathrm{a}}=0.1 \mathrm{~mm}^{-1}\right)[45,46]$.

\subsubsection{Image reconstruction}

The PA signals after filtering (3rd order Butterworth bandpass filter with 0.15 and 1.5 MHz cutoff frequency) are fed into the k-Wave package for image reconstruction using time-reversal [47-49]. A single speed of sound (SOS) value is assigned to the entire acoustic domain. The SOS value is optimized during reconstruction iteration to be $1495 \mathrm{~m} / \mathrm{s}$ for the best image quality. The images are reconstructed with a 0.5 $\mathrm{x} 0.5 \times 0.5 \mathrm{~mm}^{3}$ voxel size and interpolated for better visualization. 


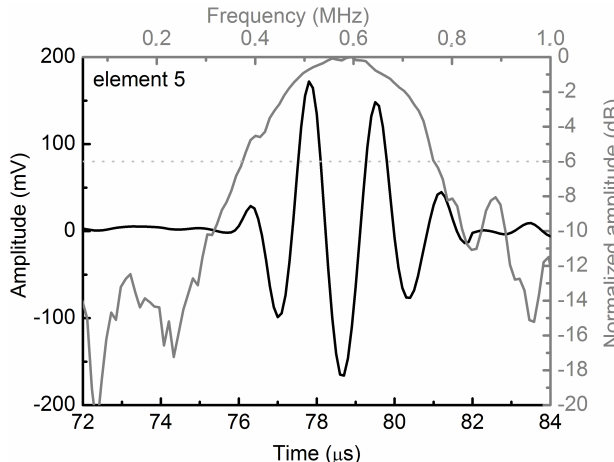

(a)

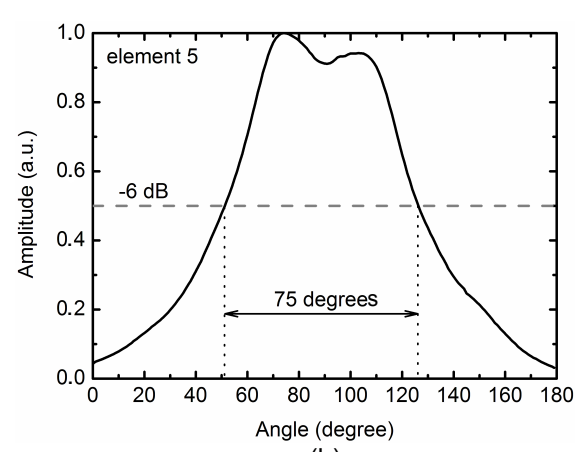

(b)

Figure 5.4: (a) Pulse-echo and frequency response, and (b) directional sensitivity of the detector element 5 .

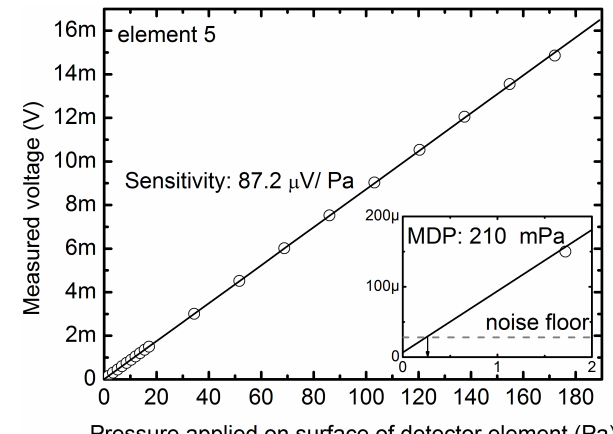

(a)

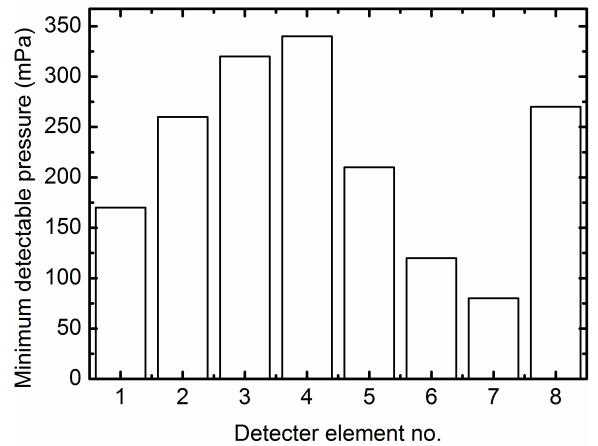

(b)

Figure 5.5: (a) Sensitivity and minimum detectable pressure of the detector element 5. (b) Minimum detectable pressure for each element in the detector array.

\section{$5.3 \quad$ Results}

\subsubsection{Detector array performance}

Figure 5.4(a) shows the measured pulse-echo and frequency response of element 5 . The element with acoustic lens has a center frequency and fractional bandwidth of $0.6 \mathrm{MHz}$ and $\sim 75 \%$, respectively. The measured directional sensitivity of the element 5 is shown in Figure 5.4(b). The directivity angle is enlarged to be around $75^{\circ}$ by the acoustic lens, which is sufficient for full breast imaging.

The measured sensitivity and MDP for element 5 are $87.2 \mu \mathrm{V} / \mathrm{Pa}$ and $210 \mathrm{mPa}$ as shown in Figure 5.5(a). MDP variations in all elements in the array are shown 
in Figure 5.5(b). The mean \pm standard deviation MDP of the array is $221 \pm 93$ $\mathrm{mPa}$. To our knowledge, this is the lowest reported MDP measured for a detector in photoacoustic breast imaging. The MDP variations are mainly due to the noise variations present for the DAQ channels. The measured MDP using this method takes the full noise frequency spectrum into account. However, in practice, when signal de-noising is applied, the MDP value can be reduced.

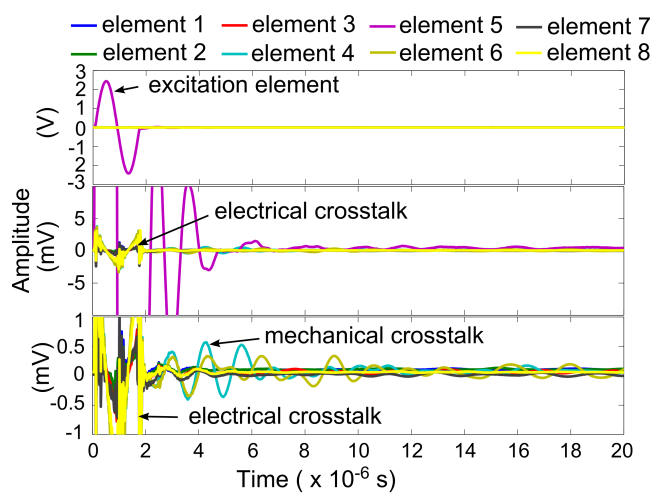

(a)

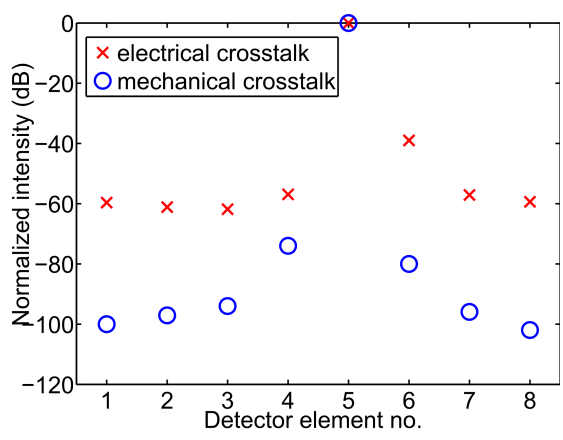

(b)

Figure 5.6: (a) Responses of all elements in the array displayed in different vertical scales for visualization. Electrical and mechanical crosstalk are well separated in time. (b) Peak-peak interelement electrical and mechanical crosstalk of the linear array detector relative to the driven element.

The results of inter-element electrical and mechanical crosstalk are shown in Figure 5.6 with the responses of all elements in Figure 5.6(a). The responses are plotted in three grouped subplots with different Y-axis units for better visualization. The strong excitation signal for element 5 can be clearly seen from the upper plot, while the responses from the other elements are not visible. The electrical and mechanical crosstalk can be identified from two zoom-in plots at middle and bottom. The peak to peak voltage of the measured electrical and mechanical crosstalk for all the elements are compared to the driving element response in Figure 5.6(b). A low mechanical crosstalk level can be observed which is possibly due to the additional air kerf separation between the sub-diced small units for each element.

\subsubsection{Spatial resolution of the system}

Phantom 1 is dedicated for the system resolution study. A top view maximum intensity projection (MIP) image of the phantom extracted from the $3 \mathrm{D}$ reconstruction is 


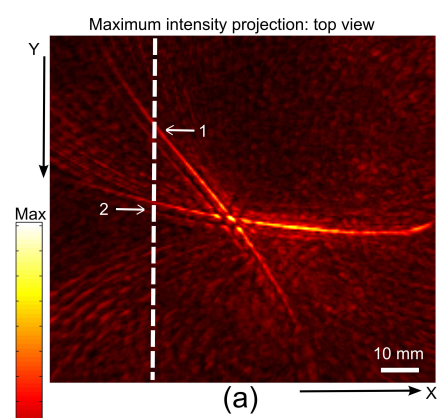

Image slice from dashed line indicated in (a)

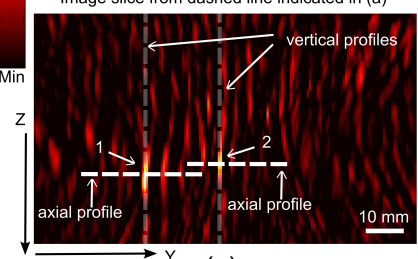

(c)
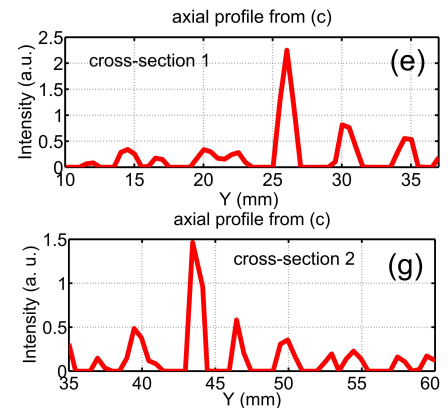

Maximum intensity projection: side view

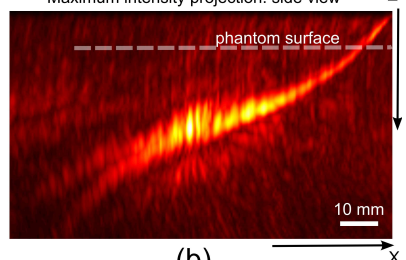

(b)

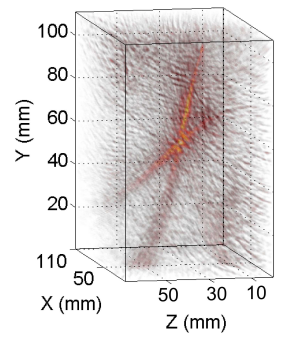

(d)

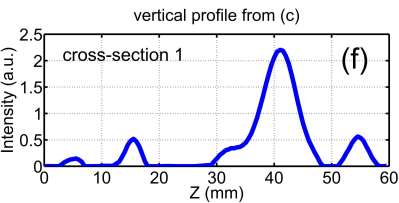

vertical profile from (c)

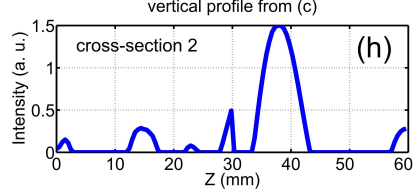

Figure 5.7: Reconstruction of phantom 1. (a) A top view maximum intensity projection (MIP) along the vertical axis (Z direction) with $100 \times 100 \mathrm{~mm}^{2}$ field of view. (b) A side view MIP along the $\mathrm{Y}$ direction with $60 \times 100 \mathrm{~mm}^{2}$ field of view. (c) An image slice at position indicated by dashed line in (a) showing cross-sections of the two threads (marked with "1", and "2") at around $4 \mathrm{~cm}$ from the phantom surface. (d) A 3D rendering of the phantom showing a $110 \times 110 \times 70 \mathrm{~mm}^{3}$ field of view. (e) An axial profile crossing a sub-resolution object ("1") from (c) indicated by a dashed white line, and (f) A vertical profile crossing a sub-resolution object ("1") from (c) indicated by a dashed gray line. (g) An axial profile crossing a sub-resolution object ("2") from (c) indicated by a dashed white line, and (h) A vertical profile crossing a sub-resolution object ("2") from (c) indicated by a dashed gray line.

shown in Figure 5.7(a). The image has a $100 \times 100 \mathrm{~mm}^{2}$ FOV. A side view MIP of the phantom is shown in Figure 5.7(b) with a $60 \times 100 \mathrm{~mm}^{2}$ FOV. The crossed-threads object is successfully visualized. A 3D rendering of the phantom with a $110 \mathrm{x} 110 \mathrm{x}$ $70 \mathrm{~cm}^{3}$ FOV is shown in Figure 5.7(d). 
We analyzed the spatial resolution of the system in the XY plane (axial and lateral) and $\mathrm{Z}$ direction (vertical). A image slice at the location indicated by a white dashed line in Figure 5.7(a) is taken from the 3D reconstruction. This is depicted in Figure 5.7(c) showing cross-sections of the two threads at around $40 \mathrm{~mm}$ from the phantom surface. Axial and lateral profiles are taken from the thread cross-sections indicated by dashed lines in Figure 5.7(c), and shown in Figure 5.7(e) and (f) for cross-section " 1 ", and (g) and (h) for cross-section "2", respectively. The full width at half maximum values of axial and lateral profiles indicate that the system has a 2 $\mathrm{mm}$ spatial resolution in the XY plane and $6 \mathrm{~mm}$ in $\mathrm{Z}$ direction at locations around $40 \mathrm{~mm}$ from phantom surface.

\subsubsection{Sensitivity and imaging quality of the system}

Phantom 2 possesses a background $\mu_{\mathrm{s}}^{\prime}=0.7 \mathrm{~mm}^{-1}$ to mimic normal breast tissue scattering, and objects with different sizes possessing $\mu_{\mathrm{a}}$ for either tumor tissue or blood vessel absorption located at various depths. This phantom was designed to evaluate the sensitivity and imaging quality of the system. A top view and a side view MIP image of the phantom are shown in Figure 5.8(a) and (b), respectively. Figure 5.8(c) and (d) are the XY plane image slices at 20 and $40 \mathrm{~mm}$ depth from the phantom surface. All objects are successfully visualized including a blood vessel mimicking object (object 1) down to a depth of $4 \mathrm{~cm}$, a 2-mm-diameter spherical object with blood absorption (object 2) at $20 \mathrm{~mm}$ depth, a 5-mm-diameter tumor mimicking spherical object (object 4) at $20 \mathrm{~mm}$ depth, and two 10-mm-diameter tumor mimicking objects (object 3 and 5) at 20 and $40 \mathrm{~mm}$ depths. Details of the background and object optical properties, object size, location are listed in Table 5.1.

\subsubsection{Field-of-view of the system}

Figure $5.7(\mathrm{~d})$ shows a $110 \times 110 \times 70 \mathrm{~mm}^{3} \mathrm{FOV}$ of the $3 \mathrm{D}$ reconstructed phantom 1 . Even larger $-6 \mathrm{~dB}$ FOV of around $170 \times 170 \times 170 \mathrm{~mm}^{3}$ can be estimated from the $-6 \mathrm{~dB}$ acceptance angle of the detector element $\left(75^{\circ}\right.$, Figure $\left.5.4(\mathrm{~b})\right)$.

\subsection{Discussion}

We presented the design and evaluated the performance of a laboratory prototype system for photoacoustic breast tomography. The detector characterization shows that the detector array possesses a mean MDP of $221 \mathrm{mPa}$, which is lower than 


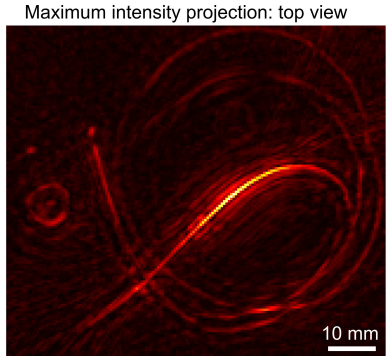

(a)

Slice at $20 \mathrm{~mm}$ from phantom surface

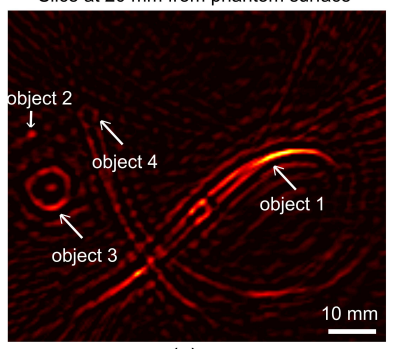

(c)

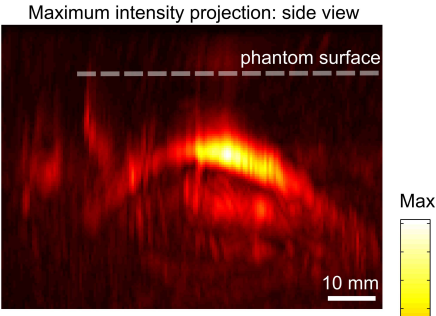

(b)

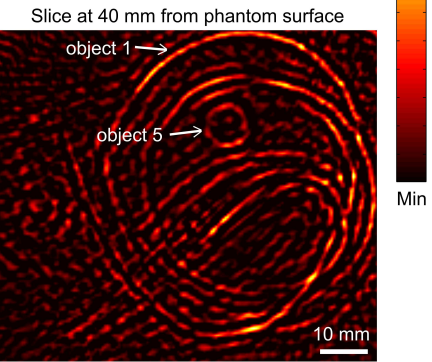

(d)

Figure 5.8: Reconstruction of phantom 2. (a) A top view maximum intensity projection (MIP) along the vertical axis (Z direction) with $70 \times 70 \mathrm{~mm}^{2}$ field of view. (b) A side view MIP along the $\mathrm{X}$ direction with $60 \times 70 \mathrm{~mm}^{2}$ field of view.(c) A XY plane image slice at $20 \mathrm{~mm}$ depth from the phantom surface showing the reconstructed objects on this plane. (d) A XY plane image slice at 40 $\mathrm{mm}$ depth from the phantom surface showing the reconstructed objects on this plane.

our previously reported value $(500 \mathrm{mPa})$ [36]. This is probably due to the improved electrical grounding and shielding of the detector and a different DAQ system used. The detector has a $0.6 \mathrm{MHz}$ center frequency with a $75 \%$ fractional bandwidth, which can be increased by using two or more front matching layers for future systems. The acceptance angle of the detector is enlarged by the lenses to be around $75^{\circ}$ at the detector center frequency. The large acceptance angle of the detector ensures a large FOV of the system.

The imaging performance of the system was evaluated using phantom experiments. The resulting $2 \mathrm{~mm}$ XY plane resolution determined by the bandwidth of the detector is sufficient for breast imaging. PA breast imaging is based on the tissue optical contrast due to vascularization, which is enhanced around a tumor due to angiogenesis [50]. This process is reported to go through two phases separated by the "angiogenic switch". Exponential tumor growth ensues in the second phase (vascular phase), which occurs from tumor sizes of 1-2 $\mathrm{mm}$ in diameter [51]. The $6 \mathrm{~mm}$ resolution in the $\mathrm{Z}$ direction (vertical resolution) is poor. This is due to the limited 
detector coverage of the phantoms in this direction. We cover only $45 \mathrm{~mm}$ of the 120 $\mathrm{mm}$ high phantom, due to the 5 scanning steps of the $40 \mathrm{~mm}$ long detector array. Further increasing the detector coverage in $\mathrm{Z}$ direction (see further) will improve the vertical resolution towards the bandwidth-determined $2 \mathrm{~mm}$ resolution.

Though image artifacts are presence, a blood vessel mimicking object down to a $40 \mathrm{~mm}$ depth and a tumor mimicking object at $40 \mathrm{~mm}$ depth were successfully visualized, indicating a good sensitivity of the imager. Objects with 1-2 mm size (object 1 and 2) are faithfully recovered with well preserved shapes, while larger objects (object 3-5) are reconstructed with edge enhancement. This is due to the finite bandwidth of our detector (Figure 5.4(a)), which does not cover the required low frequencies for PA signals generated from large objects. This observation matches the simulation results reported in Ref. [36]. The artifacts could be mitigated by deconvolution algorithms and iterative, model-based image reconstructions which will be implemented in the future. However, breast tumors are known to be heterogeneous with a scattered distribution of small absorbing regions, which makes our system well suited for sensitive clinical breast imaging due to the ultra-high sensitivity of the detector (Figure 5.5) [36].

Our system FOV $\left(170 \times 170 \times 170 \mathrm{~mm}^{3}\right)$ is considerably larger than that of Kruger's system $\left(64 \times 64 \times 50 \mathrm{~mm}^{3}\right)$ [17]. As reported by Huang et al [52], the mean breast diameter and length for the bra cup size D was measured to be $137 \mathrm{~mm}$ and $97 \mathrm{~mm}$, the FOV of our system is then suitable for 3D full breast imaging purpose.

A clinical version prototype system PAM-II will be developed based on the current system design. However, various design aspects can be further improved to address the above limitations.

\subsubsection{Light delivery}

The output laser energy delivered by the fiber bundle is around $80 \mathrm{~mJ}$, which is spread out to cover a $13 \mathrm{~cm}$ diameter phantom surface, resulting in a $0.6 \mathrm{~mJ} \mathrm{~cm}^{-2}$ fluence at phantom surface. This intensity is far under the maximum permissible exposure for near infrared light [53].

However, $80 \mathrm{~mJ}$ is the maximum output energy that can be handled by the fiber bundle while the maximum energy from the laser output is around $300 \mathrm{~mJ}$. For the future clinical version, the patient will lie in a prone position on a patient bed with her breast pendant through an aperture. The output laser beam can be split spitted into two parts, one part $(100 \mathrm{~mJ})$ can be coupled into the fiber bundle, the other part 
(200 mJ) can be guided using mirrors and expanded to illuminate the beast from the bottom.

In this design, 3 times more laser energy is delivered to the breast with more homogeneous light distribution compared to the current system. It is expected to further improve the sensitivity of our system into a greater stage.

\subsubsection{Ultrasound detection}

The vertical resolution of the current system $(6 \mathrm{~mm})$ is limited by the curtailed $\mathrm{Z}$ direction coverage due to the use of a single array with limited $\mathrm{Z}$ direction scanning. For the clinical version, more detector arrays require to be stacked in the vertical direction in a detector arm. The detector arm can be arranged to curve along the contour of the pendant breast from chest wall to the nipple. With more detector elements in the vertical direction the $\mathrm{Z}$ axis resolution will be improved to that determined by the detection bandwidth, giving an isotropic spatial resolution of $2 \mathrm{~mm}$.

Further, a multiple of such detector arms will be developed to be positioned around the breast. This will reduce the number of scanning positions around the breast. By this, the acquisition time of 1 hour for a full volume in the clinical prototype will be considerably reduced. Further, instead of the current 8 channel DAQ system, a 128 channel DAQ system under development will be used. A measuring time of under 10 minutes is aimed for.

\subsubsection{Multi-modality imaging system}

The experiments were performed using phantoms with SOS close to water. However, the breast tissue in general has higher SOS than the coupling water, and with a heterogeneous distribution [54]. To have an accurate PA reconstruction, knowledge of SOS distribution in the breast is required [55]. This can be obtained in the clinical version by placing a passive element (absorbing sphere) in front of each fiber bundle output to generate ultrasound pulses $[56,58]$. The method permits the co-registration of SOS, acoustic attenuation (AA) and optical absorption in a PA imager without the addition of extra active ultrasound generating elements [57].

Further SOS and AA distributions in the breast have been proven to have diagnostic values since malignancies have higher SOS and AA values with respect to the healthy tissues [54]. 


\subsection{Conclusions}

We presented a design and evaluation of a laboratory prototype system for 3D full breast imaging. The system is based on an optimized sensitive ultrasound detector array comprising 8 elements specifically developed for this purpose. Acoustic lenses are used to enlarge the acceptance angle of each large surface area element to ensure a wide system field of view. We characterized the detector array performance in terms of frequency response, directional sensitivity, minimum detectable pressure and inter-element electrical and mechanical cross-talk. We evaluated the performance of this laboratory prototype system using well-defined tissue mimicking phantoms. The system has a $2 \mathrm{~mm}$ XY plane resolution and a $6 \mathrm{~mm}$ vertical resolution. A vasculature mimicking object was successfully visualized down to a depth of $40 \mathrm{~mm}$ in a breast phantom. Further, tumor mimicking spherical objects with 5 and $10 \mathrm{~mm}$ diameter at $20 \mathrm{~mm}$ and $40 \mathrm{~mm}$ depths were recovered, indicating a good sensitivity of the system. The large acceptance angle of the detector ensures a 170 x $170 \times 170 \mathrm{~mm}^{3}$ system field of view, which is well suited for photoacoustic full breast tomography. We finally provide various recommendations to guide this laboratory prototype towards a clinical version with an isotropic spatial resolution of $2 \mathrm{~mm}$. 


\section{Bibliography}

[1] A. Jemal, F. Bray, M. M. Center, J. Ferlay, E. Ward, and D. Forman, "Global cancer statistics," CA Cancer J. Clin. 61(2), 69-90 (2011).

[2] D. R. Youlden, S. M. Cramb, N. A. M. Dunn, J. M. Muller, C. M. Pyke, and P. D. Baade, "The descriptive epidemiology of female breast cancer: An international comparison of screening, incidence, survival and mortality," Cancer Epidemiology 36, 237-248 (2012).

[3] W. A. Berg, L. Gutierrez, M. S. Nessaiver, W. B. Carter, M. Bhargavan, R. S. Lewis, and O. B. Loffe "Diagnostic accuracy of mammography, clinical examination, US, and MR imaging in preoperative assessment of breast cancer," Radiology 233, 830-849, (2004)

[4] C. D. Lehman, C. Isaacs, M. D. Schnall, E. D. Pisano, S. M. Ascher, P. T. Weatherall, D. A. Bluemke, D. J. Bowen, P. K. Marcom, D. K. Armstrong, S. M. Domchek, G. Tomlinson, S. J. Skates and C. Gatsonis, "Cancer yield of mammography, MR, and US in high-risk women: Prospective multi-institution breast cancer screening study," Radiology 244, 381-388, (2007)

[5] A. I. Hagen, K. A. Kvistad, L. Maehle, M. M. Holmen, H. Aase, B. Styr, A. Vabo, J. Apold, P. Skaane and P. Moller, "Sensitivity of MRI versus conventional screening in the diagnosis of BRCA-associated breast cancer in a national prospective series," Breast 16, 367-374, (2007)

[6] T. Uematsu, S. Yuen, M. Kasami, and Y. Uchida "Comparison of magnetic resonance imaging, multidetector row computed tomography, ultrasonography, and mammography for tumor extension of breast cancer," Breast Cancer Research and Treatment 112, 461-474, (2008)

[7] B. Tromberg, B. W. Pogue, K. D. Paulsen, A. G. Yodh, D. A. Boas and A. E. Cerussi, "Assessing the future of diffuse optical imaging technologies for breast cancer management," Med. Phys. 35, 2443-2451, (2008) 
[8] D. Razansky, M. Distel, C. Vinegoni, R. Ma, N. Perrimon, R. W. Köster and V. Ntziachristos, "Multispectral opto-acoustic tomography of deep-seated fluorescent protein it in vivo," Nat. Photonics $\mathbf{3}$, 412-417, (2009)

[9] J. Jose, S. Manohar, R. G. M. Kolkman, W. Steenbergenand and T. G. van Leeuwen, "Imaging of tumor vasculature using Twente photoacoustic systems," J. Biophoton 2, 701-717, (2009)

[10] L. V. Wang, "Multiscale photoacoustic microscopy and computed tomography," Nat. photonics 3, 503-509, (2009)

[11] D. Razansky, A. Buehler, and V. Ntziachristos, "Volumetric real-time multispectral optoacoustic tomography of biomarkers," Nat. Protocols 6 , 1121-1129(2011). doi:10.1038/nprot.2011.351

[12] J. Gamelin, A. Maurudis, A. Aguirre, F. Huang, P. Guo, L. V. Wang, and Q. Zhu, "A real-time photoacoustic tomography system for small animals," Opt. Express 17(13), 10489-10498, (2009)

[13] L. V. Wang and S. Hu, "Photoacoustic tomography: in vivo imaging from organelles to organs", Science 335,(2012). (doi:10.1126/science.1216210)

[14] P. Beard, "Biomedical photoacoustic imaging," Interface Focus 1, 602-631, (2011)

[15] L. Xiang, B. Wang, L. Ji, and H. Jiang, "4-D photoacoustic tomography," Scientific reports 3 , 1113, (2012) doi:10.1038/srep01113

[16] D. Piras, W. Xia, W. Steenbergen, T. G. van Leeuwen and S. Manohar, "Photoacoustic imaging of the breast using the Twente Photoacoustic Mammoscope: Present status and future perspectives," IEEE J. Sel. Topic Quantum. Electron. 16 , 730-739, (2010)

[17] R. A. Kruger, R. B. Lam, D. R. Reinecke, S. P. D. Rio and R. P. Doyle, "Photoacoustic angiography of the breast," Med. Phys. 37, 6096-6100, (2010)

[18] S. A. Ermilov, T. Khamapirad, A. Conjusteau, M. H. Leonard, R. Lacewell, K. Mehta, T. Miller and A. A. Oraevsky, "Laser optoacoustic imaging system for detection of breast cancer," J. Biomed. Opt. 14(2), 024007, (2009) 
[19] T. D. Khokhlova, I. M. Pelivanov, V. V. Kozhushko, A. N. Zharinov, V. S. Solomatin and A. A. Karabutov, "Optoacoustic imaging of absorbing objects in a turbid medium: ultimate sensitivity and application to breast cancer diagnostics," Applied Opt. 46, 262-272, (2007)

[20] Y. Wang, T. N. Erpelding, L. Jankovic, Z. Guo, J. Robert, G. David and L. V. Wang, "In vivo three-dimensional photoacoustic imaging based on a clinical matrix array ultrasound probe," J. Biomed. Opt. 17(6), 061208, (2012)

[21] F. Ye, S. Yang, and D. Xing, "Three-dimensional photoacoustic imaging system in line confocal mode for breast cancer detection," Appl. Phys. Lett. 97, 213702, (2010) doi:http://dx.doi.org/10.1063/1.3518704

[22] M. Pramanik, G. Ku, C. Li and L. V. Wang, "Design and evaluation of a novel breast cancer detection system combining both thermoacoustic (TA) and photoacoustic (PA) tomography," Med. Phys. 35, 2218-2223, (2008)

[23] H. Ke, T. D. Erpelding, L. Jankovic, C. Liu, and L. V. Wang, "Performance characterization of an integrated ultrasound, photoacoustic, and thermoacoustic imaging system," J. Biomed. Opt. 17(5), 056010, (2012)

[24] M. Heijblom, D. Piras, W. Xia, J.C.G. van Hespen, J.M. Klaase, F.M. van den Engh, T.G. van Leeuwen, W. Steenbergen, and S. Manohar, "Visualizing breast cancer using the Twente photoacoustic mammoscope: What do we learn from twelve new patient measurements?," Opt. Express 20, 11582-11597, (2012) (doi: http://dx.doi.org/10.1364/OE.20.011582)

[25] L. Xi, X. Li, L. Yao, S. Grobmyer, and H. Jiang,"Design and evaluation of a hybrid photoacoustic tomography and diffuse optical tomography system for breast cancer detection," Med. Phys. 39, 2584-2594 , (2012)

[26] Z. Xie, X. Wang, R. F. Morris, F. R. Padilla, G. L. Lecarpentier, and P. L. Carson, "Photoacoustic imaging for deep targets in the breast using a multichannel 2D array transducer," SPIE Proceedings, 7899, 1172-1181, (2011)

[27] V. G. Andreev, A. A. Karabutov and A. A. Oraevsky, "Detection of ultrawideband ultrasound pulses in optoacoustic tomography," IEEE Trans. Ultrason. Ferr. Freq. Contr. 50, 1383-1390 (2003). 
[28] A. Oraevsky, V. G. Andreev, A. A. Karabutov, S. V. Solomatin, E. V. Savateeva, R. D. Fleming, Z. Gatalica, and H. Singh, "Laser optoacoustic imaging of breast cancer in vivo," Proc. SPIE 4256, 6Ü15 (2001).

[29] R. A. Kruger, K. D. Miller, H. E. Reynolds, W. L. Kiser, D. R. Reinecke and G. A. Kruger, "Breast cancer in vivo: Contrast enhancement with thermoacoustic CT at 434 MHz-feasibility study," Radiology 216, 279-283 (2000).

[30] H. Jiang, "Diffuse Optical Tomography: Principles and Applications," (CRC, Florida, 2011).

[31] R. A. Kruger, W. L. Kiser, D. R. Reinecke, G. A. Kruger, and K. D. Miller, "Thermoacoustic optical molecular imaging of small animals," Mol. Imaging 2(2), 113-123, (2003).

[32] S. Manohar, A. Kharine, J. C. G. van Hespen, W. Steenbergen, and T. G. van Leeuwen, "Photoacoustic mammography laboratory prototype: Imaging of breast tissue phantoms," J. Biomed. Opt. 9, 1172-1181 (2004).

[33] S. Manohar, S. E. Vaartjes, J. C. G. van Hespen, J. M. Klaase, F. M. van den Engh, W. Steenbergen, and T. G. van Leeuwen, "Initial results of in vivo noninvasive cancer imaging in the human breast using near-infrared photoacoustics," Opt. Express 15, 12277-12285 (2007).

[34] B. J. Tromberg, N. Shah, R. Lanning, A. Cerrusi, J. Espinoza, T. Pham, L. Svaasand, and J. Butler, "Non invasive in vivo characterization of breast tumors using photon migration spectroscopy," Neoplasia 2, 26-40 (2000).

[35] P. Taroni, A. Bassi, D. Comelli, A. Farina, R. Cubeddu, and A. Pifferi, "Diffuse optical spectroscopy of breast tissue extended to 1100 nm," J. Biomed. Opt. 14, 054030 (1999).

[36] W. Xia, D. Piras, J. C. G. van Hespen, S. van Veldhoven, C. Prins, T. G. van Leeuwen, W. Steenbergen, and S. Manohar,"An optimized ultrasound detector for photoacoustic breast tomography," Med. Phys. 40(3), 032901 (2013).

[37] W. Xia, D. Piras, J. C. G. van Hespen, W. Steenbergen, and S. Manohar,"A new acoustic lens material for large area detectors in photoacoustic breast tomography," Photoacoustics 1(2), 9-18 (2013). (doi: http://dx.doi.org/10.1016/j.pacs.2013.05.001) 
[38] J. Callerama, R. H. Tancrell and D. T. Wilson, "Transmitters and receivers for medical ultrasonics" Ultrasonic Symposium Proceedings, IEEE CH14829/79/0000-0407, 407-411, (1979)

[39] P. L. M. J. van Neer, G. Matte, M. G. Danilouchkine, C. Prins, F. van den Adel and N. de Jong, "Super-harmonic imaging: development of an interleaved phase-array transducer," IEEE Trans. Ultrason. Ferr. Freq. Contr. 57, 455-468 (2010). (doi: 10.1109/TUFFC.2010.1426)

[40] J. M. Cannata, J. A. Williams, Q. Zhou, T. A. Ritter and K. K. Shung, "Development of a 35-MHz piezo-composite ultrasound array for medical imaging," IEEE Trans. Ultrason. Ferr. Freq. Contr. 53(1), 224-236 (2006).

[41] H. J. Van Staveren, C. J. M. Moes, J. van Marie, S. A. Prahl and M. J. C. van Gemert, "Light scattering in Intralipid-10\% in the wavelength range of 400-1100 nm," Appl. Opt. 30, 4507-4514, (1991).

[42] W. Xia, D. Piras, M. Heijblom, W. Steenbergen, T. G. van Leeuwen and S. Manohar, "Poly(vinyl alcohol) gels as photoacoustic breast phantoms revisited," J. Biomed. Opt. 16(7), 075002, (2011).

[43] J. A. Curcio and C. C. Petty, "The near infrared absorption spectrum of liquid water", J. Acoust. Soc. Am. 41(5), (1951), 302-304

[44] F. Martelli and G. Zaccanti "Calibration of scattering and absorption properties of a liquid diffusive medium at NIR wavelengths. CW method," Opt. Express 15(2), 486-500, (2007).

[45] L. Spinelli, A. Torricelli, A. Pifferi, P. Taroni, G. M. Danesini, and R. Cubeddu "Bulk optical properties and tissue components in the famale breast from multiwavelength time-resolved optical mammography" J. Biomed. Opt. 9(6), 1137$1142,(2004)$.

[46] A. Roggan, M. Friebel, K. Dorschel, A. Hahn and G. Muller "Optical properties of circulating human blood in the wavelength range 400-2500 nm," J. Biomed. Opt. 4(1), 36-46, (1999).

[47] B.T. Cox and P.C. Beard, "Fast calculation of pulsed photoacoustic field in fluids using k-space metholds," J. Acoust. Soc. Am. 117(6), 3616-3627, (2005) 
[48] B. E. Treeby, and B. Cox, "k-Wave: MATLAB toolbox for the simulation and reconstruction of photoacoustic wave fields," J. Biomed. Opt. 51(2), 021314, (2010)

[49] B. E. Treeby, E. Z. Zhang and B. Cox, "Photoacoustic tomography in absorbing acoustic media using time reversal," Inverse Problems 26, 115003, (2010)

[50] J. Folkman, "Tumor angiogenesis," in Cancer Medicine, J. F. Holland, Ed., 5th ed. Hamilton, ON: B. C. Decker, 2000, ch. 9, pp. 132 Ü152.

[51] G. Bergers and L. E. Benjamin, "Tumorigenesis and the angiogenic switch," Nat. Rev. Cancer. 3, 401-410 (2003). (doi: 10.1038/nrc1093)

[52] S. Huang, J. M. Boone, K. Yang, N. J. Packard, S. E. McKenney, N. D. Prionas, K. K. Lindfors and M. J. Yaffe, "The characterization of breast anatomical metrics using dedicated breast CT," Med. Phys. 38(4), 2180-2190 (2011).

[53] ANSI, Z136.1-2007.

[54] C. Li, N. Duric, P. Littrup, and L. Huang, "In vivo breast sound-speed imaging with ultrasound tomography," Ultrasound Med. Biol. 35, 1615-1628, (2009).

[55] J. Jose, R. G. H. Willemink, W. Steenbergen, T. G. van Leeuwen and S. Manohar, "Speed-of-sound compensated photoacoustic tomography for accurate imaging," Med. Phys. 39, 7262-7271 (2012).

[56] S. Manohar, R. G. H. Willemink, F. van der Heijden, C. H. Slump, and T. G. van Leeuwen, "Concomitant speed-of-sound tomography in photoacoustic imaging," Appl. Phys. Lett. 91, 131911, (2007).

[57] J. Jose, R. G. H. Willemink, S. Resink, D. Piras, J. C. G. van Hespen, C. H. Slump, W. Steenbergen, T. G. van Leeuwen and S. Manohar, "Passive element enriched photoacoustic computed tomography (PER PACT) for simultaneous imaging of acoustic propagation properties and light absorption," Opt. Express 19(3), 2093-2104 (2011).

[58] S. A. Ermilov, A. Conjusteau, T. Hernandez, R. Su, V. Nadvoretsky, D. Tsyboulski, F. Anis, M. A. Anastasio, and A. A. Oraevsky, "3D laser optoacoustic ultrasonic imaging system for preclinical research," Proc. SPIE 8581, 85810N, (2013). 



\section{Chapter 6}

\section{Conclusions and outlook}

\subsection{The ultrasound detector}

\subsubsection{Bandwidth and sensitivity}

Sensitive and faithful detection of PA signals is of crucial importance for PA breast imaging. Imaging at large depths $(5+\mathrm{cm})$ required in breast imaging represents a great challenge mainly due to the strong light attenuation in breast tissue, which requires sensitive US detectors. Further frequency dependent acoustic attenuation (AA) of breast tissue heavily attenuates high frequencies of US waves, shifts the spectrum of the initiated PA signals to lower regions. Thus, the defining characteristics of PA imagers such as resolution, imaging depth and image contrast are predominantely determined by US detector performances such as sensitivity, center frequency and bandwidth.

Photoacoustic signal generation and propagation has been described in detail by Hoelen and de Mul [1]. For a sufficiently short laser pulse, in thermal and stress confinement, the photoacoustic signal from a spherical object detected at distance $z$ can be expressed as [2]:

$$
P(z, t)=-P_{\max } \frac{\sqrt{2 e} c}{r_{0}}\left(t-\frac{z}{c}\right) \exp \left\{-\left[\frac{c}{r_{0}}\left(t-\frac{z}{c}\right)\right]^{2}\right\}
$$

in which

$$
P_{\max }=\frac{\Gamma E_{a}}{(2 \pi)^{3 / 2} \sqrt{e} r_{0}^{2} z}
$$

where $c$ is speed of sound of the medium, $r_{0}$ is the radius of the spherical object, $E_{a}$ is the absorbed light energy by the object, $\Gamma$ is the efficiency of photoacoustic conversion 
or the Grüneisen coefficient. When the size of the object is much smaller than the inverse of its absorption coefficient $\left(1 / \mu_{a}\right)$, and the measurements are performed in a backward detection mode, the absorbed energy can be expressed as [3,4]:

$$
E_{a}=\mu_{a} V_{\mathrm{obj}} \Phi_{0} \exp \left[-\mu_{\mathrm{eff}}(z)\right]
$$

in which, $V_{\text {obj }}$ is the volume of the absorbing object, $\Phi_{0}$ is the fluence at the tissue surface (maximum $20 \mathrm{~mJ} \mathrm{~cm}^{-2}$ in the visible wavelengths is used), $\mu_{\text {eff }}$ is the effective optical attenuation coefficient of the medium. Substitution of Equation (6.3) into Equation (6.2) results in:

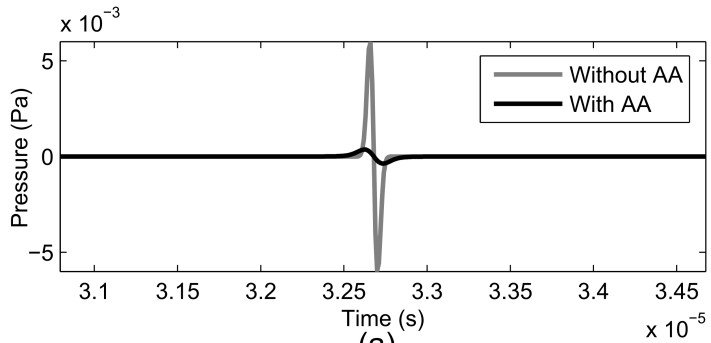

(a)

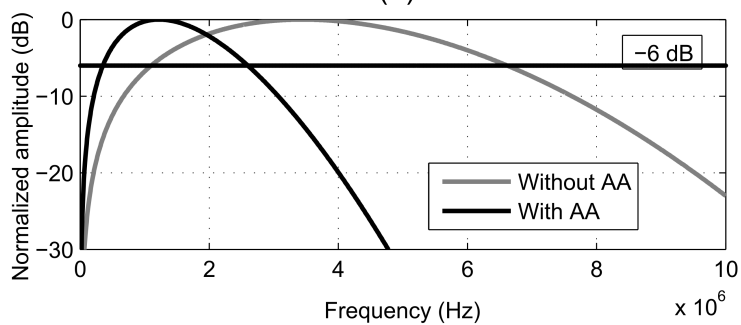

(b)

Figure 6.1: Photoacoustic signal arriving at tissue surface from a $100 \mu \mathrm{m}$ spherical object (blood vessel contrast) at $5 \mathrm{~cm}$ depth with and without the taking into account of acoustic attenuation (AA). (a) Time domain signals. (b) Normalized spectrums.

$$
P_{\max }=\frac{2 \Gamma \mu_{a} r_{0} \Phi_{0}}{3 z \sqrt{2 \pi}} \exp \left[-\mu_{\mathrm{eff}}(z)\right]
$$

To simulate the loss in ultrasound propagation, the frequency dependent acoustic attenuation in tissue requires to be incorporated into the photoacoustic wave Equation (6.1), the photoacoustic signals arriving at the detector can be expressed as [5]:

$$
P_{\text {att }}(z, t)=\operatorname{IFFT}\{\operatorname{FFT}[P(z, t)] A(f)\}
$$


in which,

$$
A(f)=\exp \left(-\alpha_{0} f^{n} z\right)
$$

where $\alpha_{0}$ is the acoustic attenuation coefficient at $1 \mathrm{MHz}$ and $n$ is the power law factor.

Table 6.1: Optical, acoustic and thermo-elastic properties of healthy breast tissue and tumors used in the photoacoustic signal generation and propagation model. Optical properties are from Ref. [6] at $785 \mathrm{~nm}$ wavelength. The speed of sound, acoustic attenuation and optical scattering properties, and Grüneisen coefficient are assumed to be homogenously distributed in the model used. Healthy tissue, tumor and blood are modelled to have differences in optical absorption only.

\begin{tabular}{|c|c|c|c|}
\hline Properties & Healthy tissue & Tumor & Blood vessel \\
\hline $\begin{array}{l}\text { Speed of sound }(\mathrm{c}) \\
\left(\mathrm{m} \mathrm{s}^{-1}\right)\end{array}$ & 1530 & - & - \\
\hline $\begin{array}{l}\text { Acoustic attenuation }{ }^{\mathrm{a}}\left(\alpha_{0} f^{n}\right) \\
\left(\mathrm{dB} \mathrm{cm}^{-1}\right)\end{array}$ & $0.75 \mathrm{f}^{1.5}$ & - & - \\
\hline $\begin{array}{l}\text { Optical absorption }\left(\mu_{a}\right) \\
\left(\mathrm{cm}^{-1}\right)\end{array}$ & 0.04 & 0.08 & $1.0^{\mathrm{b}}$ \\
\hline $\begin{array}{l}\text { Optical reduced scattering }\left(\mu_{s}^{\prime}\right) \\
\left(\mathrm{cm}^{-1}\right)\end{array}$ & 12 & - & - \\
\hline $\begin{array}{l}\text { Optical effective attenuation }\left(\mu_{e f f}\right) \\
\left(\mathrm{cm}^{-1}\right)\end{array}$ & 1.2 & - & - \\
\hline Grüneisen coefficient $^{\mathrm{c}}(\Gamma)$ & - & 0.8 & 0.8 \\
\hline
\end{tabular}

a Ref. [7].

b At $800 \mathrm{~nm}$, derived from Ref. [8].

c Ref. [9].

To study the frequency contents and peak pressures of arriving PA signals from the breast arriving at the surface, we considered the PA signals from tumors with various diameters from $1 \mathrm{~mm}$ to $10 \mathrm{~mm}$, and feeding blood vessels with diameters ranging from 0.1 to $1 \mathrm{~mm}$, assuming a $20 \mathrm{~mJ} \mathrm{~cm}^{-2}$ radiant exposure at $800 \mathrm{~nm}$ at tissue surface. The optical and acoustic properties of breast tissues used in the simulations are listed in Table 6.1. 

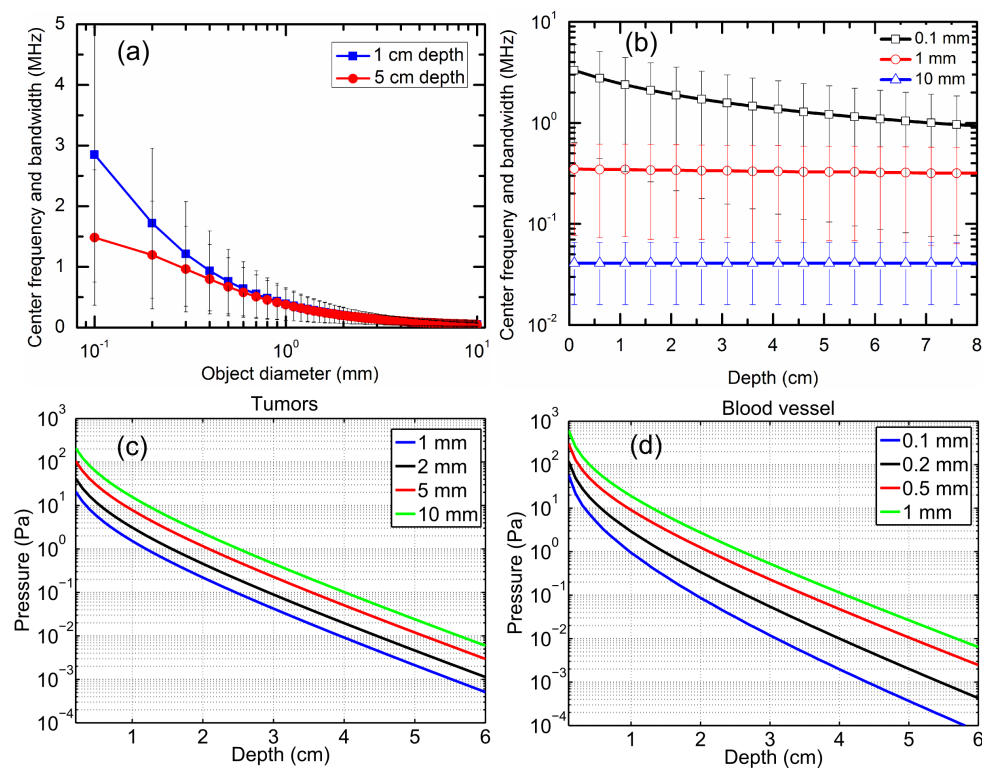

Figure 6.2: Characteristics of photoacoustic signals arriving at tissue surface from spherical objects with different diameters, located at different depths. Center frequency and bandwidth for (a) spherical objects with diameters from $0.1-10 \mathrm{~mm}$ located at 10 and $50 \mathrm{~mm}$ depth and (b) Object with diameters of $0.1,1$, and $10 \mathrm{~mm}$ located at various depth from tissue surface to $80 \mathrm{~mm}$. Solid lines represent center frequencies and error bars represent half bandwidths. (c) Peak pressures for tumors with diameter of $1 \mathrm{~mm}, 2 \mathrm{~mm}, 5 \mathrm{~mm}$ and $10 \mathrm{~mm}$. (d) Peak pressures for blood vessels approximated by spheres with diameter of $0.1 \mathrm{~mm}, 0.2 \mathrm{~mm}, 0.5 \mathrm{~mm}$ and $1 \mathrm{~mm}$.

Figure 6.1 gives an example of the photoacoustic signal generated from a 100 $\mu \mathrm{m}$ spherical object at $5 \mathrm{~cm}$ depth with blood vessel contrast arriving at the tissue surface. The signal is heavily attenuated due to the frequency dependent acoustic attenuation. The amplitude of the signal decreases by a factor of ten from several $\mathrm{mPa}$ to less than $1 \mathrm{mPa}$ (Figure 6.1(a)). The peak frequency of the signal shifts from $4 \mathrm{MHz}$ to around $1 \mathrm{MHz}$ (Figure 6.1(b)).

Figure $6.2(\mathrm{a})(\mathrm{b})$ show center frequency and $-6 \mathrm{~dB}$ bandwidth of the PA signals from spherical objects with different diameters $(0.1 \mathrm{~mm}$ to $10 \mathrm{~mm})$ and located at different depths (tissue surface to $80 \mathrm{~mm}$ ). Solid lines represent center frequencies and error bars represent half bandwidths. For $10 \mathrm{~mm}$ depth, the center frequencies from all light absorbing objects are less than $3 \mathrm{MHz}$, while maximum frequencies are less than $5 \mathrm{MHz}$ due to frequency dependent $\mathrm{AA}$, indicating the maximum frequency of the generated PA signal from the breast absorbing structures at $10 \mathrm{~mm}$ depth is less than $5 \mathrm{MHz}$. The center frequency and maximum frequency decrease with increase of 
object depth. For $50 \mathrm{~mm}$ depth, the maximum center frequency shifts to be around 1.5 MHz, while the maximum frequency decreases to $\sim 2.5 \mathrm{MHz}$. For tumors with $1 \mathrm{~mm}$ diameter, which is reported to be the size for tumors to initiate exponential growth due to flipping of the angiogenic switch [10], the maximum frequency at all depth are less than $1 \mathrm{MHz}$. This suggests that an ultrasound detector with a bandwidth covering from a few tens of $\mathrm{kHz}$ upto $\sim 1 \mathrm{MHz}$ is required to encompass all US frequencies from tumors and from a few hundreds of $\mathrm{kHz}$ to $2.5 \mathrm{MHz}$ is required to cover the most US frequency contents from the feeding blood vessels. For early detection of breast cancer, the ultrasound detector is required to be able to detect tumors larger than $2 \mathrm{~mm}$ diameter. The photoacoustic signals originating from those $2+\mathrm{mm}$ diameter tumors have maximum frequency lower than $1 \mathrm{MHz}$ as shown in Figure 6.2, which makes our detector bandwidth (single-element detector in Chapter 3) well-suited.

Figure 6.2 (c) and (d) show the peak pressures of the arriving PA signals from spherical objects with different diameters and located at different depths for tumors and blood vessels respectively. The peak pressure decreases with decrease in object size and with increase in object depth as expected. At $5 \mathrm{~cm}$ depth, the generated signals from $2 \mathrm{~mm}$ tumors are extremely weak $(\sim 5 \mathrm{mPa})$, which requires an extremely sensitive ultrasound detector. However, it is less challenging to detect signals from large blood vessels ( $1 \mathrm{~mm}$ at $5 \mathrm{~cm}$ depth) which shows a peak pressure of $\sim 30 \mathrm{mPa}$ due to the higher optical contrast. This indicates that a minimum detector pressure (MDP) of $\sim 5 \mathrm{mPa}$ is required for US detectors in PA mammography to detect the early stage of malignant tumors.

The predicted extremely weak peak pressures $(5 \mathrm{mPa})$ from $2 \mathrm{~mm}$ diameter tumors indicate the challenge for photoacoustics used for breast imaging. The MDP value of our detector (single-element detector in Chapter 3) is around $500 \mathrm{mPa}$, which is still worse than the required $5 \mathrm{mPa}$. However, using multiple projections and signal averaging, the noise floor is reduced by the square root of the number of averages and projections. In this case, considering a laser with repetition rate of $10 \mathrm{~Hz}$, each signal is averaged 100 times, detection of $5 \mathrm{mPa}$ pressure from a $2 \mathrm{~mm}$ tumor requires 100 projections for a single element detector, which takes $\sim 17$ min. For real-time breast imaging for applications such as visualizing blood flow dynamics using Indocyanine green as contrast agent, parallel data acquisition (DAQ) is required.

The bandwidth of our detector is $80 \%$, which can be increased by using two or more front matching layers in future. The image quality of our detector can be improved with the use of a deconvolution operation to compensate for finite bandwidths effects. 
Objects larger than $\sim 5 \mathrm{~mm}$ generating US frequencies below the bandwidth of our detector are expected to be reconstructed with edge-enhancement. However, breast tumors are known to be heterogeneous with a scattered distribution of absorbing regions. In such a case this detector is still well suited to faithfully image a collection of small absorbing structures, which makes up the tumor mass.

\subsubsection{Directivity}
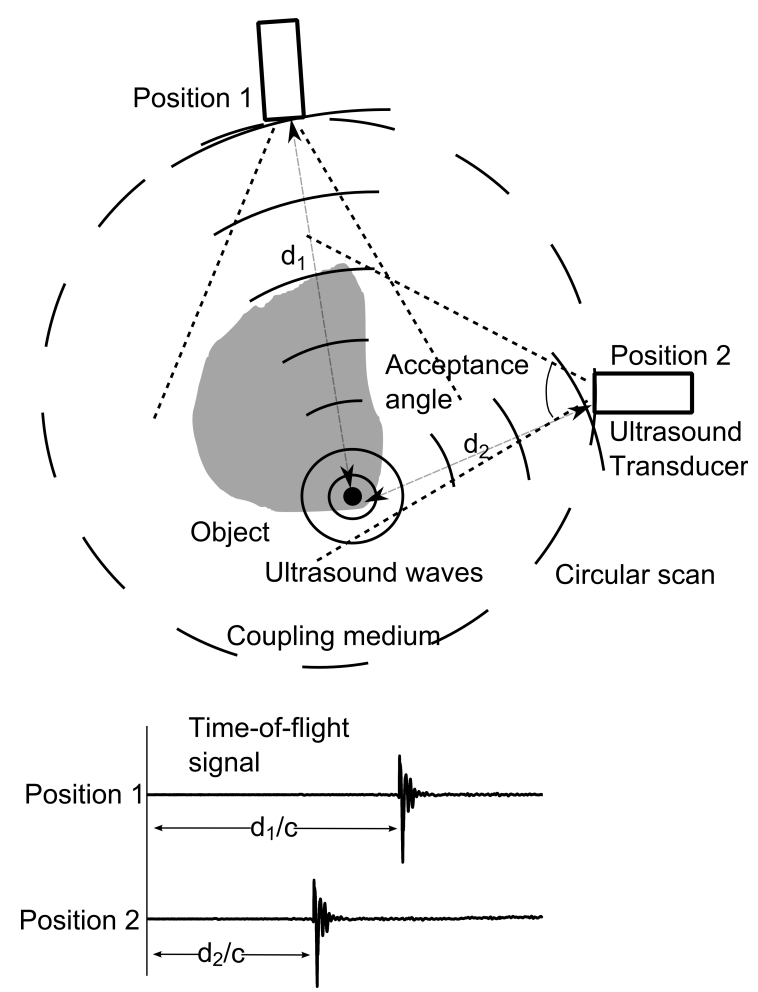

Figure 6.3: Schematics of a 2D photoacoustic tomography system showing the necessity for the acceptance angle to encompass the object for coherent signals detection from all angular position in performing reconstruction.

In photoacoustic tomography, the breast is illuminated with short-pulsed laser light and the ultrasound detector scans the object ideally over $360^{\circ}$. A requirement in this geometry is that each US detectors's acceptance angle is wide enough to detect photoacoustic signals generated throughout the entire object for each detector angular 
position around the object (Figure 6.3). In this condition, PA signals in the breast are entirely registered for each detection position. During a backprojection style reconstruction, the coherent signals from all detector angular positions are summed to form an image of the photoacoustic sources. The lateral resolution and image contrast are both improved when this requirement is fulfilled (Chapeter 4).

The directivity angle of our detector with a Stycast 1090SI acoustic lens attached on top is around $75^{\circ}$, the field-of-view (FOV) of the photoacoustic breast tomography system can be estimated to be around $170 \times 170 \times 170 \mathrm{~mm}^{3}$, considering the detector is situated $110 \mathrm{~mm}$ away from the rotation center. This FOV is large enough to encompass most breast sizes according to Ref. [11](Chapter 5).

\subsection{Acoustic lens}

Owing to its excellent acoustic properties, the proposed acoustic lens material Stycast 1090SI is a good candidate to enlarge the acceptance angle of a large surface area, sensitive ultrasound detector. Consequently, the lateral resolution of the imaging system will be improved without introducing additional ultrasound internal reflection induced imaging artifacts (Chapter 4).

However, there are a few drawbacks that require to be addressed when using this acoustic lens:

1) Care needs to be taken to prevent air bubbles entrapped inside the material during material manufacturing. This might be a challenge for mass production and requires good quality control.

2) The center frequency and bandwidth of the detector is lowered due to the frequency dependent AA of the lens material. It is important to take this into account when designing the center frequency of ultrasound detectors. However, the resulting detector with this lens used in photoacoustic tomography experiments confirmed that the bandwidth determined resolution is around $2 \mathrm{~mm}$, which is sufficient for detecting early breast cancer (Chapter 4 and 5).

3) The insertion loss for PMMA lens is smaller than for the Stycast 1090SI lens. This is because the acoustic impedance of PMMA lies between water and the front matching layer of the detector, which makes PMMA function as a second impedance matching layer between the detector front matching layer and surrounding water, increasing the detector sensitivity. For the Stycast 1090SI, due to the large difference between the acoustic impedance of the detector front matching layer and the lens material, the reflection loss is large. To prevent this loss for Stycast 1090SI lens, 
the acoustic impedance of the detector front matching layer requires to be tuned to be lower than the present value. Detectors with two or more front matching layers with acoustic impedance of the outer matching layer close to tissue will minimize the acoustic reflection loss induced by the Stycast 1090SI lens, and further increase the detector bandwidth.

\subsection{Recommendations for future PAMs}

Chapter 5 reported a laboratory prototype system based on the previously developed detector (Chapter 3) and acoustic lenses (Chapter 4). The system has a $2 \mathrm{~mm} \mathrm{XY}$ plane resolution and a $6 \mathrm{~mm}$ vertical resolution. A vasculature mimicking object was successfully visualized down to a depth of $40 \mathrm{~mm}$ in a breast phantom. Further, tumor mimicking spherical objects with 5 and $10 \mathrm{~mm}$ diameters at $20 \mathrm{~mm}$ and 40 $\mathrm{mm}$ depths were recovered, indicating a good sensitivity of the system. The large acceptance angle of the detector ensures a $170 \times 170 \times 170 \mathrm{~mm}^{3}$ system field of view, which is well suited for photoacoustic full breast tomography. However, various design aspects can be further improved for the future versions of the Twente Photoacoustic Mammoscope.

\subsubsection{Excitation}

The $755 \mathrm{~nm}$ excitation wavelength is chosen for its high optical absorption contrast between tumor and normal tissue, while $1064 \mathrm{~nm}$ wavelength is selected for the large optical penetration depth in breast tissue. Further since the optical absorption spectrum of deoxy-hemoglobin has a local peak at $755 \mathrm{~nm}$, using this wavelength will facilitate the tumor diagnosis by revealing the hypoxia in tumor neovasculature. However, more wavelengths are required to exploit the the capability of multispectral photoacoustics including the differentiation of the abundance of specific tissue components in the breast and the quantification of oxygen saturation of hemoglobin [12].

However, the large pulse energy required for photoacoustic breast imaging prohibits the use of multispectral photoacoustics since traditional Nd:YAG laser pumped optical parametric oscillator (OPO) provides a pulse energy below $100 \mathrm{~mJ}$ in the socalled near-infrared (NIR) window (600-1000 nm) for biological tissue. The energy gap will be shortened in future with either the achievement of higher energy lasers or the achievement of higher sensitivity detectors. However, the detector developed in Chapter 3 has paved the way for clinical application of multispectral photoacoustic 


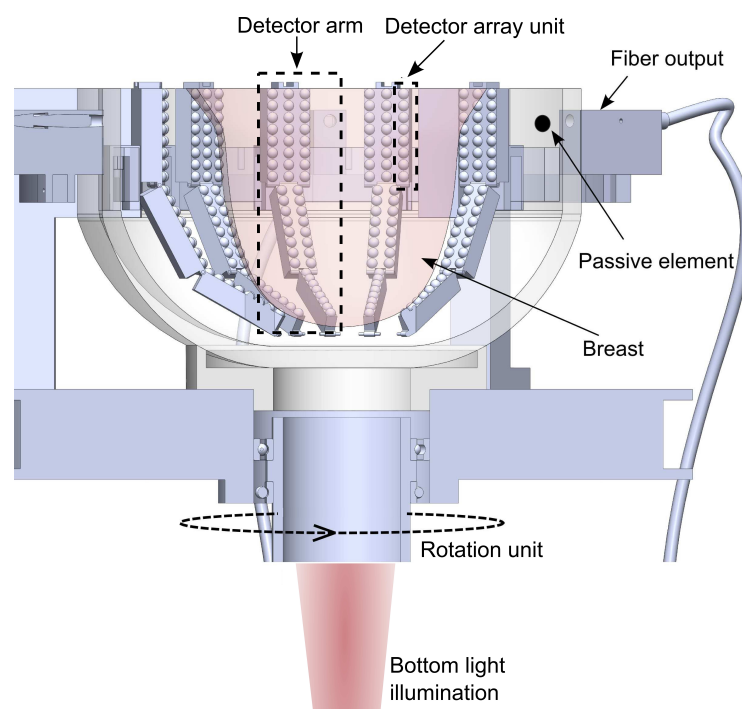

Figure 6.4: Schematic showing the prospects of the future clinical version system.

breast tomography due to its unprecedented high sensitivity, which requires a laser pulse energy of only $80 \mathrm{~mJ}$ (Chapter 5).

\subsubsection{US arrays}

The optimized US detector has only a single front matching layer, resulting a $80 \%$ fractional bandwidth. Combined with the Stycast 1090SI lens, the detector has a $70 \%$ bandwidth. The sensivity and bandwidth of the detector with acoustic lens can be improved by using two or more front matching layers. However, this requires the redesign of the detector matching layers including the selection of material acoustic impedances and their thicknesses.

The limited vertical resolution of laboratory prototype system described in Chapter $5(6 \mathrm{~mm})$ can be improved by increasing the angle of detection view, for example as shown in Figure 6.4. Each detector arm can have 6 detector array units with each unit being the same as the linear array employed in the laboratory prototype system (Chapter 5). The detector arms can be arranged to match the contour of the breast surface. Thus a larger detection view can be achieved to approach the bandwidth-determined $2 \mathrm{~mm}$ resolution in all directions. 


\subsubsection{Multi-modality imaging system}

The PAT experiments in Chapter 5 were performed using phantoms with SOS close to water. However, the breast tissue in general has larger SOS than the coupling water, and further with a heterogeneous distribution [13]. To have an accurate PA reconstruction, knowledge of SOS distribution in the breast under PA examination is required [14]. This can be obtained by placing a passive element (absorbing sphere) in front of each fiber bundle output to generate ultrasound pulses (Figure 6.4) [15]. This method permits the co-registration of SOS, acoustic attenuation and optical absorption in a PA imager without the addition of extra active ultrasound generating elements [16].

Further SOS and AA distributions in the breast have been proven to have diagnostic values since malignancies have higher SOS and AA values with respect to the healthy tissues [13].

\subsection{Outlook}

The future roles of photoacoustic breast imaging in clinical practice will strongly depend on the outcome of clinical case studies. Sensitivity and specificity of PAMs need to be evaluated in comparison with X-ray mammography and MRI. With tremendous promise, PAM could supplement X-ray mammography for use as diagnosis tools, or if possessing sufficient sensitivity and specificity, could potentially replace X-ray mammography for breast cancer screening. 


\section{Bibliography}

[1] C. G. Hoelen, and F. F. M. de Mul, "A new theoretical approach to photoacoustic signal generation," J. Acoust. Soc. Am., 106(2), 695-706 (1999).

[2] M. W. Sigrist and F. K. Kneubühl, "Laser generated stress waves in liquids," J. Acoust. Soc. Am., 64, 1652-1663 (1978).

[3] E. A. Profio, "Light trasport in tissue," Appl. Opt., 28, 2216-2222 (1989).

[4] S. Manohar, T. G. van Leeuwen, J. M. Klaase, F. M. Van den Engh, and W. Steenbergen, "Photoacoustic mammography with a flat detection geometry," in Photoacoustic Imaging and Spectroscopy, L. V. Wang, Ed. Boca Raton, FL: CRC Press, pp. 431-442, (2009).

[5] T. J. Allen, and P. C. Beard, "Optimising the detection parameters for deep tissue photoacoustic imaging," Proc. of SPIE, 8223,82230P, (2012).

[6] L. Spinelli, A. Torricelli, A. Pifferi, P. Taroni, G. M. Danesini, and R. Cubeddu, "Bulk optical properties and tissue components in the famale breast from multiwavelength time-resolved optical mammography," J. Biomed. Opt., 9(6), 11371142, (2004).

[7] T. L. Szabo, "Diagnostic Ultrasound Imaging," Elsevier Academic Press: London, (2004).

[8] A. Roggan, M. Friebel, K. Dörschel, A. Hahn, and G. Müller, "Optical properties of circulating human blood in the wavelength range 400-2500 nm," J. Biomed. Opt., 4(1), 36-46, (1999).

[9] A. A. Oraevsky, and A. A. Karabutov, "Time-resolved detection of optoacoustic profiles for measurement of optical energy distribution in tissues," in Handbook of Optical Biomedical Diagnostics, V. V. Tuchin, Ed. Bellingham, WA: SPIE Press, pp 585-646, (2002). 
[10] G. Bergers and L. E. Benjamin, "Tumorigenesis and the angiogenic switch," Nat. Rev. Cancer., 3, 401-410 (2003). (doi: 10.1038/nrc1093)

[11] S. Huang, J. M. Boone, K. Yang, N. J. Packard, S. E. McKenney, N. D. Prionas, K. K. Lindfors and M. J. Yaffe, "The characterization of breast anatomical metrics using dedicated breast CT," Med. Phys. 38(4), 2180-2190 (2011).

[12] D. Razansky, A. Buehler, and V. Ntziachristos, "Volumetric real-time multispectral optoacoustic tomography of biomarkers," Nat. Protocols 6, 1121-1129, (2011) (doi:10.1038/nprot.2011.351)

[13] C. Li, N. Duric, P. Littrup, and L. Huang, "In vivo breast sound-speed imaging with ultrasound tomography," Ultrasound Med. Biol., 35, 1615-1628, (2009).

[14] J. Jose, R. G. H. Willemink, W. Steenbergen, T. G. van Leeuwen and S. Manohar, "Speed-of-sound compensated photoacoustic tomography for accurate imaging," Med. Phys., 39, 7262-7271 (2012).

[15] S. Manohar, R. G. H. Willemink, F. van der Heijden, C. H. Slump, and T. G. van Leeuwen, "Concomitant speed-of-sound tomography in photoacoustic imaging," Appl. Phys. Lett., 91, 131911, (2007).

[16] J. Jose, R. G. H. Willemink, S. Resink, D. Piras, J. C. G. van Hespen, C. H. Slump, W. Steenbergen, T. G. van Leeuwen and S. Manohar, "Passive element enriched photoacoustic computed tomography (PER PACT) for simultaneous imaging of acoustic propagation properties and light absorption," Opt. Express, 19(3), 2093-2104 (2011). 


\section{Summary}

The standard modality for breast cancer detection is X-ray imaging. Diagnosis is performed after the triple assessment of X-ray mammography assisted by ultrasonography and biopsy. Magnetic resonance imaging (MRI) is sometimes used in specific problem solving such as contradictory results are obtained from X-ray and ultrasound images. X-ray mammography is capable of producing 2D projection images with a high spatial resolution. However, X-ray mammography besides possessing ionizing hazards, is less sensitive in women with dense breasts. In addition, X-ray mammography has difficulties to image tumors close to the chest wall. Ultrasonography still suffers from poor soft tissue contrast, inherent speckle noise, strong operator dependence and lack of standardization. MRI has high sensitivity but suffers from variable specificity, a relatively high cost and needs the use of a contrast agent. Thus there is a great need for an alternative technology to detect and diagnose early stages of breast cancer with high sensitivity and specificity.

Photoacoustic (PA) imaging has shown great potential to visualize high optical absorption contrast based on hemoglobin absorption that can impact breast cancer detection and diagnosis. State-of-the-art photoacoustic breast imaging systems are promising but are limited either by only a $2 \mathrm{D}$ imaging capability or an insufficient imaging field-of-view (FOV) in 3D. This thesis investigates various aspects regarding the design and development of a sensitive 3D photoacoustic tomography system for full breast imaging, focusing on the optimization of ultrasound detection, the heart of the system.

Chapter 1 reviews the system performance and first clinical experiences of the Twente Photoacoustic Mammoscope (PAM), an earlier 3D imager developed in our group, in comparison with the current PA breast imagers. Though clinical case studies are promising, PAM suffers from a limited FOV, a limited imaging depth associated with a flat plate geometry and single projection view with one-sided illumination, and a relatively low sensitivity of the detector. To further explore the capabilities of PA in breast cancer detection and diagnosis, a second version PAM is motivated.

Tissue mimicking phantoms are important for the evaluation of system performance characteristics, such as resolution, sensitivity and imaging depth. In Chapter 2, a popular phantom in PA imaging, poly(vinyl alcohol) (PVA) hydrogel fabricated by freezing and thawing (F-T) aqueous solutions of PVA, is studied for the suitability for use as PA breast phantoms. Earlier work studied the optical and acoustic 
properties of the gels in small test specimens where temperature is relatively homogeneously distributed. In a breast-sized sample we observed substantial temperature differences between the shallow regions and the interior during the F-T procedure. The spatial distribution of optical and acoustic properties were measured at various locations in the sample and correlated to the microstructure examined. The spatial distribution of speed of sound and acoustic attenuation were relatively uniform, while the reduced optical scattering coefficient showed significant differences between the surface and the bulk. This distribution fortuitously matches the optical properties of the two-layer breast model used in x-ray dosimetry with an inner region composed of an adipose tissue-glandular tissue mixture, and an outer layer of predominantly adipose tissue. We conclude that large PVA samples prepared using the standard recipe make excellent breast phantoms.

Chapter 3 presents the design and evaluation of a specialized single-element ultrasound detector for photoacoustic breast tomography. We first present design considerations such as the required detector sensitivity and its frequency response, a selection of active material and matching layers and their geometries, which lead to a functional model. By iteration between simulation of detector performances, fabrication and experimental characterization of functional models an optimized implementation is made and evaluated. The effect of lateral resonances was addressed and their influence minimized by sub-dicing the samples. The final optimized detector has a center frequency of $1 \mathrm{MHz}$ and a $-6 \mathrm{~dB}$ bandwidth of $0.4-1.25 \mathrm{MHz}$ (fractional bandwidth of $80 \%$ ). The detector is capable of detecting vascularized tumors with 1-2 $\mathrm{mm}$ resolution. The measured minimum detectable pressure is $0.5 \mathrm{~Pa}$, which is the lowest reported in literature for a detector in photoacoustic breast imaging.

In Chapter 4, we propose a new acoustic lens material based on the 2-component resin Stycast 1090SI in comparison with the commonly used material acrylic plastic (PMMA) to enlarge the acceptance angle of sensitive large surface area detectors, thus improving lateral resolution. We characterized the acoustic properties of the proposed material in comparison with PMMA. Detector performance using negative lenses with the two materials, was tested using finite element simulation and experiment. Further the image quality of a photoacoustic tomography system was studied using k-Wave simulation and experiment. Our acoustic characterization showed that Stycast 1090SI has tissue-like acoustic impedance, high speed of sound and low acoustic attenuation. Both acoustic lenses show significant enlargement of detector acceptance angle and lateral resolution improvement. However, image artifacts induced by acoustic lenses are reduced using the Stycast 1090SI lens compared to PMMA lens. 
In Chapter 5, an 8-element linear ultrasound detector array is developed based on the single-element ultrasound detector presented in Chapters 3 and 4. A laboratory prototype system is designed and evaluated for 3D photoacoustic tomography with the optimized ultra-high sensitivity detector array. We evaluated the performance of the system using breast sized tissue mimicking phantoms with absorbing inclusions mimicking blood vessels and tumors. The resulting system exhibits a $2 \mathrm{~mm} \mathrm{XY}$ plane resolution and a $6 \mathrm{~mm}$ vertical resolution. A vasculature mimicking object is successfully visualized down to a depth of $40 \mathrm{~mm}$ in the phantom. Further, tumor mimicking spherical objects with 5 and $10 \mathrm{~mm}$ diameter at $20 \mathrm{~mm}$ and $40 \mathrm{~mm}$ depths are recovered, indicating good sensitivity of the system. The system has a $170 \mathrm{x} 170$ $\mathrm{x} 170 \mathrm{~mm}^{3}$ field of view, which is well suited for full breast imaging.

Chapter 6 introduces criteria for the sensitivity and bandwidth of ultrasound detectors for PA breast imaging by analyzing the PA signals arriving at the detection site in simulations, leading to the discussion and conclusions of this thesis. Various recommendations are provided to guide the development of the future instruments. 


\section{Samenvatting}

De medische standaard voor borstkanker screening is X-ray mammografie. De uiteindelijke diagnose wordt gesteld aan de hand van drie verschillende methoden samen. Hierbij wordt X-ray mammografie ondersteund door echografie en histologie. Magnetic resonance imaging of MRI wordt zo nu en dan gebruikt wanneer de resultaten van X-ray en echografie elkaar tegenspreken. X-ray mammografie is instaat om 2D projecties te maken met een hoge spatiale resolutie. Naast de gevaren van de gebruikte ioniserende straling is X-ray mammografie ook minder gevoelig of nauwkeurig bij vrouwen met compact borstweefsel. Tumoren die dicht bij de borstkast zitten zijn daarnaast ook moeilijk in beeld te krijgen. Echografie lijd nog altijd aan een slecht visueel contrast tussen zachte weefsels, granulaire ruis (speckle noise), een sterke afhankelijkheid van de gebruiker en een gebrek aan standaardisering. MRI heeft een hoge gevoeligheid, maar lijd aan een variabele specificiteit, relatief hoge kosten en gebruikt invasieve contrast middelen. Kortom, er is een grote vraag naar een alternatieve techniek waarmee je zo vroeg mogelijk en met een hoge gevoeligheid en specificiteit borstkanker kan detecteren en diagnosticeren.

Fotoakoestische (PA) beeldvorming is een veelbelovende techniek met een hoog optisch contrast dat verzaakt wordt door absorptie van licht door hemoglobine. Deze techniek kan een grote invloed hebben op borstkanker detectie en diagnostiek. De huidige grensverleggende generatie van borst imaging systemen zijn veel belovend, maar worden gelimiteerd door alleen te beschikken over 2D beeldvorming of 3D beeldvorming met een gelimiteerde zichtveld (field-of-view, FOV). Dit proefschrift beschrijft verscheidene aspecten omtrent het ontwerpen en ontwikkelen van een gevoelig fotoakoestisch systeem voor borstkanker detectie in de hele borst. Hierbij ligt de nadruk op het optimaliseren van de ultrageluid detectie: Het hart van het systeem.

Hoofdstuk 1 vergelijkt de prestaties en klinische ervaringen van de "Twente Photoacoustic Mammoscoop" (PAM), een 3D systeem dat eerder in onze groep is ontwikkeld, met de huidige PA borst imagers. Ondanks dat de klinische studies veel belovend zijn doet het PAM systeem onder aan de andere systemen door de gelimiteerde FOV, de beperkte gevoeligheid van de detector en een beperkte meetdiepte die door wordt veroorzaakt door de platte plaat geometrie en de enkelzijdige belichting. Hieruit blijkt dat, bij vervolg onderzoek naar het mogelijke gebruik van PA voor detectie en diagnostisering van borstkanker, een nieuwe verbeterde versie van PAM nodig is. 
Weefsel nabootsende materialen, genaamd fantomen, zijn belangrijk voor de evaluatie van de systeem eigenschappen zoals resolutie, gevoeligheid en meetdiepte. In hoofdstuk 2 wordt het in de PA populaire materiaal poly (vinyl alcohol) (PVA) hydrogel bestudeerd als mogelijk fantoom materiaal voor het nabootsen van de borst. PVA hydrogel kan gemaakt worden door een proces van vriezen en dooien (Freeze and Thaw, F-T) van PVA in een waterige oplossing. Eerdere studies beschreven de optische en akoestische eigenschappen van de hydrogelen in kleine test monsters waarbij de temperatuur relatief homogeen verspreid was. In een monster ter grootte van een borst observeerden wij daarentegen substantiële temperatuur verschillen tussen de gebieden aan de buiten en binnenkant van het fantoom tijdens het F-T proces. De ruimtelijke distributie van de geluidssnelheid en akoestische verzwakking waren relatief uniform door heel het materiaal. De zogenaamde "reduced optical scattering coëfficiënt", een maat voor afstandsafhankelijke verstrooiing van licht, liet daarentegen significant grote verschillen tussen de binnen- en buitenkant van het materiaal zien. Toevalligerwijze komt dit verschil in verstrooiingscoëfficiënt overeen met de optische eigenschappen van het uit twee lagen bestaande borst model dat gebruikt wordt in X-ray dosimetry. Hierbij bestaat de binnenkant van de borst uit een mengsel van vetweefsel en klierweefsel en de buitenste laag hoofdzakelijk uit vetweefsel. We komen hierbij tot de conclusie dat het standaard recept voor het maken van kleine PVA fantomen ook zeer geschikt is voor het maken van borst fantomen.

Hoofdstuk 3 beschrijft het ontwerpen en evalueren van één enkel element van een ultrasound detector die gespecialiseerd is in fotoakoestische tomografie op borsten. Eerst worden de voorwaarden beschouwd voor het ontwerp. Belangrijke parameters hiervoor zijn de benodigde gevoeligheid en frequentierespons van de detector, een selectie van actieve materialen, de bijpassende lagen en de benodigde geometrie die samen tot een functioneel model moeten leiden. Er is een geoptimaliseerd model gemaakt en geëvalueerd door de eigenschappen van detector te simuleren, door het fabricage proces te variëren en optimaliseren en door de functionele proefmodellen uitgebreid te karakteriseren. Het effect van zijdelingse of laterale resonanties is onderzocht en aangepakt door de detector in kleinere blokken te verdelen zodat de invloed van deze resonanties minimaal is. De uiteindelijke geoptimaliseerde detector heeft een centrale frequentie van $1 \mathrm{MHz}$ en een -6dB bandbreedte van $0,4-1,25 \mathrm{MHz}$ (fractionele bandbreedte van $80 \%$ ). Deze detector is instaat om doorbloede tumoren van 1-2 mm te detecteren. The gemeten minimaal detecteerbare druk is 0.5 Pa. Dit is in de laagst gerapporteerde waarde in de literatuur voor een detector die bedoeld is voor borstkanker imaging. 
In hoofdstuk 4, stellen we een nieuw materiaal voor akoestische lenzen voor die gebaseerd is op de twee componenten hars Stycast 1090SI en vergelijken deze met de standaard, polymethylmethacrylaat (PMMA, Perspex ), die vaker gebruikt wordt om de ontvangsthoek en laterale resolutie van detectoren met grote gevoelige oppervlakken te vergroten. De akoestische eigenschappen van Stycast en PMMA worden uitgebreid vergeleken. De prestaties voor detectoren met negatieve lenzen van de geselecteerde materialen zijn getest met behulp van eindige elementen (finite element) simulaties en experimenten. Daarnaast is de beeldkwaliteit voor een PA tomografie systeem bestudeerd met behulp van $k$-Wave simulaties en experimenten. Onze akoestische karakterisering liet zien dat Stycast 1090SI een akoestische impedantie heeft die vergelijkbaar is met weefsel en daarnaast een hoge snelheid van het geluid en een lage akoestische demping. Beide akoestische lenzen laten een significante vergroting van de ontvangsthoek en verhoging laterale resolutie zien. Stycast 1090SI laat echter een vermindering van de, door de akoestische lenzen veroorzaakte, beeld artefacten zien ten opzichte van PMMA.

In hoofstuk 5, is de ontwikkeling van een lineaire ultrageluid detector met acht elementen beschreven die zijn gebaseerd op het enkele element ontwerp van de hoofdstukken 3 en 4 . Een laboratorium prototype voor 3D fotoakoestische tomografie is ontworpen en getest in combinatie met de geoptimaliseerde en daardoor zeer gevoelige ultrageluid detector. Het testen van de prestaties van dit systeem is gebeurd met behulp van weefsel imiterende fantomen ter grootte van de bost met daarin absorberende structuren die bloedvaten en tumoren nabootsen. Het systeem laat een resolutie in het XY vlak zien van $2 \mathrm{~mm}$ en een resolutie in de verticale richting van $6 \mathrm{~mm}$. Een nagebootst bloedvat is succesvol gevisualiseerd tot op een diepte van $40 \mathrm{~mm}$ in het fantoom. Daarnaast zijn tumor imiterende bolletjes met een diameter van 5 en $10 \mathrm{~mm}$ op een diepte van 20 en $40 \mathrm{~mm}$ teruggevonden wat duid op een goede gevoeligheid van het systeem. Het systeem heeft een zichtveld van 170 x 170 x $170 \mathrm{~mm}^{3}$ wat zeer geschikt is voor het afbeelden een volledige borst.

Hoofdstuk 6 presenteert de criteria voor de gevoeligheid en bandbreedte van ultrageluid detectoren voor borst imaging met behulp van fotoakoestiek. Deze zijn verkregen door de mogelijke fotoakoestische signalen die de detector zouden kunnen bereiken te simuleren. Dit alles leidt tot de discussie en conclusies van dit proefschrift. Tot slot worden er diverse aanbevelingen aangereikt met de ontwikkelingen van toekomstige systemen in het oog. 


\section{Acknowledgements}

In the past four years, so many people have helped me through the completion of this thesis. I owe my gratitude to all those people who helped me in completing this thesis.

My first sincere gratitude is to my supervisors, Prof. Wiendelt Steenbergen and Prof. Ton G. van Leeuwen, who opened the door for me to work in this wonderful group. Wiendelt, you are such a professor who always gave me great freedom to explore on my own, and at the same time guided me in right path when my steps faltered. I would also like to thank you for the letter you sent to the Chinese embassy to prove my politeness. Ton, you have been always there to give support, advice and helpful suggestions. Thanks for your tireless and careful reading and comments on this thesis.

My deep gratitude goes to Dr. Srirang Manohar, my daily supervisor. Srirang, thanks for your patient and great guidance through all these days for my PhD life. I have learned so much from you in all aspects, from scientific vision, design of experiments, to technical writing and publishing results. Thanks for spending your invaluable time to guide my research and provide useful suggestions, to correct my manuscripts on countless revisions.

I gratefully acknowledge my thesis committee members for their invaluable time and effort reviewing this thesis, especially in the period of the summer holiday.

I would like to thank Johan C. G. van Hespen for his technical support throughout all my PhD. Many of my ideas would still be on paper without his help. Thanks for his useful suggestions and creative ideas in solving various practical problems. I enjoyed the cover page that we designed.

I thank Daniele Piras for the useful discussions regarding my research during these years. Daniele, thanks for your helps for various experiments and measurements. In addition, I would like to tell you once more that I enjoyed the tiramisu and various types of cakes that you always use to share. I thank Michelle Heijblom for sharing her clinical experience for improving various design aspects for the new instrument and interesting experiences they had in clinics.

I acknowledge S. van Veldhoven, C. Prins, F. van den Adel from Oldelft Ultrasound B.V., Prof. H. Sterenborg, Dr. A. Amelink, Dr. U. Gamm from Erasmus Medical Center, Prof. K. Boller, Dr. C. Lee from laser physics group and Dr. J. Klaase, Dr. F. van den Engh from Medisch Spectrum Twente for their continuous effort making 
the project successful by close and effective collaboration.

I would like to take this opportunity to acknowledge my students Daniel Bastiaan, Rogier Wildeboer, Mithun K. A. Singh, and Guus van Raaphorst for supporting me. Special thanks to Rogier for his excellent work for realizing the idea of the harmonic photoacoustic imaging (HPI). Mithun, thanks for your preamplifer design which helped to improve the sensitivity of my detector. I also would like to thank you for those non-smoking coffee breaks and interesting discussions.

My officemate and paranymph, Steffen Resink, thanks for the time that you spend with me discussing various interesting topics. Sylvia, thanks for your helps for dealing with all those administrative work. You helped me saving a lot of time. Altaf, I would like to thank you for allowing me to use your DAQ system for use in my experiments in chapter 5, and for helping me stop smoking by giving me a bad example. Peter, thanks for your help for various experiments and useful discussions. In addition, thanks for your dutch translation of the summary of this thesis.

I must express my gratitude to Ivo, Erwin, Wilma, Khalid, Samir, Jacob, Yolanda, Pim, Diederik, Jithin, Roy, Constantin, Raja, Aliaksandr and all BMPI members who always helped me with feedbacks during the work meetings. All of you were so friendly and helpful throughout my $\mathrm{PhD}$ period.

I express my deep gratitude to all of my friends in China, Germany, Netherlands and other parts of the word. You are sources of joy and support.

Words are too simple to express my gratitude to my family...

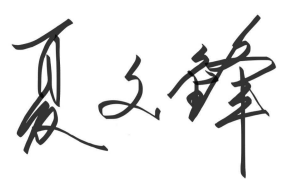

Enschede, October 2013 


\section{Curriculum vitae}

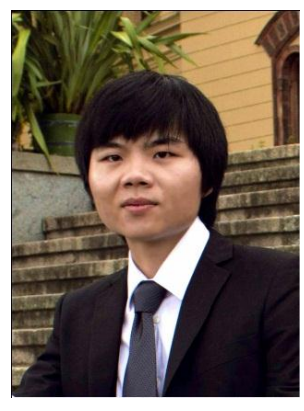

Wenfeng Xia was born on March $3^{\text {rd }}, 1983$ in Daye, Hubei province, China. He received his bachelor degree in Electrical Engineering from the Shanghai JiaoTong University, in Shanghai, China, in 2005. He obtained his master in science degree in medical physics from University of Heidelberg, in Heidelberg, Germany, in 2007. His graduation project was performed in the Mannheim Biomedical Engineering Laboratories, in Mannheim Germany, under the supervision of Prof. Josef Bille, and Prof. Markolf H. Niemz, focusing on the feasibility study of dental caries removal by picosecond and femtosecond laser ablation. After master study, he joined the Institute for Biomedical Optics, in Luebeck, Germany, working as an assistant researcher under the supervision of Prof. Reginald Birngruber and Dr. Ralf Brinkmann. He was in a research project on monitoring and automate control of the therapeutic temperature during retinal photocoagulation utilizing a photoacoustic temperature probe, where he focused on photoacoustic signal detection.

From May 2009 to May 2013, he worked as a Ph.D student in the Biomedical Photonics Imaging group, University of Twente, Enschede, the Netherlands, under the supervision of Prof. Wiendelt Steenbergen, Prof. Ton G. van Leeuwen, and Dr. Srirang Manohar. He was embedded in a research project on the development of the second generation Twente photoacoustic mammoscope based on computed tomography configuration. He focused on the design, realization and evaluation of a highly sensitive broadband detection system specialized for photoacoustic breast tomography. 



\section{List of publications}

Publications in peer-reviewed journals

1. D. Piras, W. Xia, W. Steenbergen, T. G. van Leeuwen and S. Manohar, "Photoacoustic imaging of the breast using the Twente Photoacoustic Mammoscope: Present status and future perspectives," IEEE J. Sel. Topic Quantum. Electron. 16 , 730$739,(2010)$

2. W. Xia, D. Piras, M. Heijblom, W. Steenbergen, T. G. van Leeuwen and S. Manohar, "Poly(vinyl alcohol) gels as photoacoustic breast phantoms revisited," J. Biomed. Opt. 16(7), 075002, (2011).

3. M. Heijblom, D. Piras, W. Xia, J.C.G. van Hespen, J.M. Klaase, F.M. van den Engh, T.G. van Leeuwen, W. Steenbergen, and S. Manohar, "Visualizing breast cancer using the Twente photoacoustic mammoscope: What do we learn from twelve new patient measurements?," Opt. Express 20, 11582-11597, (2012)

4. W. Xia, D. Piras, J. C. G. van Hespen, S. van Veldhoven, C. Prins, T. G. van Leeuwen, W. Steenbergen, and S. Manohar, "An optimized ultrasound detector for photoacoustic breast tomography," Med. Phys. 40(3), 032901 (2013).

5. W. Xia, D. Piras, J. C. G. van Hespen, W. Steenbergen, and S. Manohar,"A new acoustic lens material for large area detectors in photoacoustic breast tomography," Photoacoustics 1(2), 9-18 (2013).

6. W. Xia, D. Piras, M. K. A. Singh, J. C. G. Van Hespen, T. G. van Leeuwen, W. Steenbergen and S. Manohar, "Design and evaluation of a laboratory prototype system for 3D photoacoustic full breast tomography," Biomed. Opt. Express 4(11) (2013). 
Publications in conference proceedings

1. D. Piras, W. Xia, M. Heijblom, W. Steenbergen, T. G. van Leeuwen, S. Manohar, "Spatial distributions of optical and acoustic properties and correlations with temperature in cyclically frozen-thawed poly(vinyl alcohol) gel breast phantoms," Proc. SPIE 7906790600 (2011).

2. M. Heijblom, D. Piras, W. Xia, J. C. G. Van Hespen, F. Van Den Engh, J. M. Klaase, T. G Van Leeuwen, W. Steenbergen, and S. Manohar, "TU-C-220-03: Visualization of Breast Carcinoma Using Photoacoustic Imaging: The Ongoing Twente Experience" Med. Phys. 38, 3763 (2011).

3. D. Piras, W. Xia, M. Heijblom, E. M. Ten Tije, J. C. G. Van Hespen, W. Steenbergen, T. G. van Leeuwen, S. Manohar, F. Van den Engh, J. M. Klaase, "Breast imaging with the Twente Photoacoustic Mammoscope," BioPhotonics, 2011 International Workshop on , 1-3 (2011).

4. M. Heijblom, D. Piras, E. M. Ten Tije, W. Xia, J. C. G. Van Hespen, J. M. Klaase, F. Van Den Engh, T. G Van Leeuwen, W. Steenbergen, and S. Manohar, "Breast imaging using the Twente photoacoustic mammoscope (PAM): new clinical measurements" Proc. SPIE 808780870 N, (2011).

5. W. Xia, D. Piras, M. Heijblom, J. C. G. Van Hespen, S. van Veldhoven, C. Prins, T. G van Leeuwen, W. Steenbergen, S. Manohar, "Enlarged acceptance angle of a finite size detector in photoacoustic imaging using acoustic lenses," Proc. SPIE 8090 80900L, (2011).

6. W. Xia, D. Piras, M. Heijblom, J. C. G. Van Hespen, S. van Veldhoven, C. Prins, T. G van Leeuwen, W. Steenbergen, S. Manohar, "A custom-made linear array transducer for photoacoustic breast imaging," Proc. SPIE 8223 82233D (2012).

7. D. Piras, M. Heijblom, W. Xia, T. G. van Leeuwen, W. Steenbergen, S. Manohar, "Adapted directivity approach for photoacoustic imaging reconstruction," Proc. SPIE 8223 82231Z-1, (2012).

8. W. Xia, D. Piras, M. Heijblom, J. C. G. Van Hespen, S. van Veldhoven, C. Prins, T. G van Leeuwen, W. Steenbergen, S. Manohar, "FEM model based optimization 
of transducer geometry for photoacoustic imaging," in Biomedical Optics and 3-D Imaging, OSA Technical Digest, paper BSu3A.45. (2012).

9. M. Heijblom, D. Piras, W. Xia, J. C. G. Van Hespen, F. Van Den Engh, J. M. Klaase, T. G Van Leeuwen, W. Steenbergen, and S. Manohar, "Imaging breast lesions using the Twente photoacoustic mammoscope: ongoing clinical experience" Proc. SPIE 8223 82230C, (2012).

10. W. Xia, D. Piras, M. K. A. Singh, J. C. G. Van Hespen, S. van Veldhoven, C. Prins, T. G. van Leeuwen, W. Steenbergen and S. Manohar, "Design considerations for ultrasound detectors in photoacoustic breast imaging," Proc. SPIE 8581 $858113,(2013)$. 

ISBN: 978-90-365-1216-9

\section{UNIVERSITY OF TWENTE.}

